Supporting Information

\title{
Enantiodivergent Prenylation via Deconjugative Isomerization
}

\author{
Tobias Morack, ${ }^{*}$ Carina Onneken, ${ }^{\ddagger}$ Hiroshi Nakakohara, ${ }^{\ddagger}$ Christian Mück- \\ Lichtenfeld and Ryan Gilmour* \\ Organisch Chemisches Institut, Westfälische Wilhelms-Universität Münster, Corrensstraße 36, \\ 48149 Münster, Germany
}

Corresponding Author: t_mora03@uni-muenster.de and ryan.gilmour@uni-muenster.de

- General Information S2

- Experimental Section S5

Synthesis of starting materials S5

Racemic isomerization of alkenes S19

Enantioselective isomerization of $\quad$ S33

Enantioselective isomerization of
cyclic alkenes

- Mechanistic Investigations $\quad$ S54

UV/vis Spectroscopy $\quad$ S54

Reaction monitoring $\quad$ S55

Isotope labelling experiments $\quad$ S58

Kinetic isotope effect $\quad$ S59

Hammett correlations $\quad$ S63

CD-Spectrum S65

- DFT Calculations $\quad \mathrm{S66}$

- NMR Spectra S81

- References $\quad \mathrm{S} 128$ 


\section{General Information}

All chemicals were purchased as reagent grade and used without further purification. Solvents for purification (extraction and chromatography) were purchased as technical grade and distilled on the rotary evaporator prior to use. Solvents for photochemical reactions were degassed by purging with argon for $30 \mathrm{~min}$. For column chromatography $\mathrm{SiO}_{2}(40-63 \mu \mathrm{m}$ for Flash-Chromatography, VWR Chemicals) was used as stationary phase. Analytical thin layer chromatography (TLC) was performed on aluminum foil pre-coated with $\mathrm{SiO}_{2}-60 \mathrm{~F}_{254}$ (Merck) and visualized with a UV-lamp (254 nm) and $\mathrm{KMnO}_{4}$ or CAM solution. Concentration in vacuo was performed at $\sim 10$ mbar and $40{ }^{\circ} \mathrm{C}$, drying at $\sim 10^{-2}$ mbar and room temperature. NMR spectra were measured by the NMR service of the Organisch-Chemisches Institut, Westfälische Wilhelms-Universität Münster on a Bruker BZH 200/52, Bruker AV300, Bruker AV400, Agilent DD2 500 or an Agilent DD2 600 spectrometer at room temperature. The chemical shifts are referenced to the residual solvent peak as internal standard. ${ }^{[1]}$ The resonance multiplicity is abbreviated as: s (singlet), d (doublet), t (triplet), q (quadruplet), p (pentet), sext (sextet), sep (septet), m (multiplet) and b (broad). Assignments of unknown compounds are based on DEPT, COSY (HH), HMBC, HSQC and NOESY spectra. Melting points were measured on a Büchi B-545 melting-point apparatus in open capillaries. IR spectra were recorded on a Perkin-Elmer 100 FT-IR spectrometer, selected adsorption bands are reported in wavenumbers $\left(\mathrm{cm}^{-1}\right)$ and intensities are reported as: w (weak), m (medium), s (strong) and b (broad). High-resolution mass spectra (HR-ESI) were measured by the MS service of the Organisch-Chemisches Institut, Westfälische Wilhelms-Universität Münster. UV-Vis absorption spectra were measured on a Agilent Cary 60 spectrophotometer in a $1 \mathrm{~cm}$ quartz cuvette. Photochemical isomerization reactions at room temperature were performed utilizing a set-up of 4 Avonec 3 W High Power LED on star-platine 390-400 $\mathrm{nm}(U V-A)$ with a radiation angle of $120^{\circ}$ (emission spectrum see Figure S1A, general set-up see Figure S1B). The forward current per chip was set to $700 \mathrm{~mA}$ and the resulting radiant flux was $1000 \mathrm{~mW}$. The distance between the reaction vessels and the UV-lamp was set at approximately $0.5 \mathrm{~cm}$ for all reactions. Photochemical isomerization reactions at low temperatures were performed utilizing a set-up of 6 individual Inolux 5 W High Power LED's (emission maximum $395 \mathrm{~nm}$, dimmed to $3 \mathrm{~W}$ ) on a star-platine with a radiation angle of $30^{\circ}$. The forward current per chip was set to $700 \mathrm{~mA}$ and the resulting radiant flux was $1200 \mathrm{~mW}$. A custom-made quartz-glas rod was used as optical fibre. Temperature control was achieved by using a Julabo FT902 cryostat and acetone as liquid carrier. The enantiomeric ratio of the products was determined by chiral HPLC on an Agilent 1100 series (DAD, Agilent 
technologies 1200 series $)$ using a Chiracel AS-H (5 $\mu \mathrm{m}, 250.4 .6 \mathrm{~mm})$ or a Chiracel OJ-H (5 $\mu \mathrm{m}$, $250.4 .6 \mathrm{~mm}$ ) column and $n$-hexane/ $i$-propanol as eluent. Optical rotations were measured on a Perkin-Elmer 341 polarimeter. The CD-spectrum of compound (-)-12 was measured on a JASCO J-1500 CD-spectrometer in $\mathrm{CH}_{2} \mathrm{Cl}_{2}(\mathrm{c}=0.33 \mathrm{mg} / \mathrm{mL})$ and the spectrum is the average of measurements. 

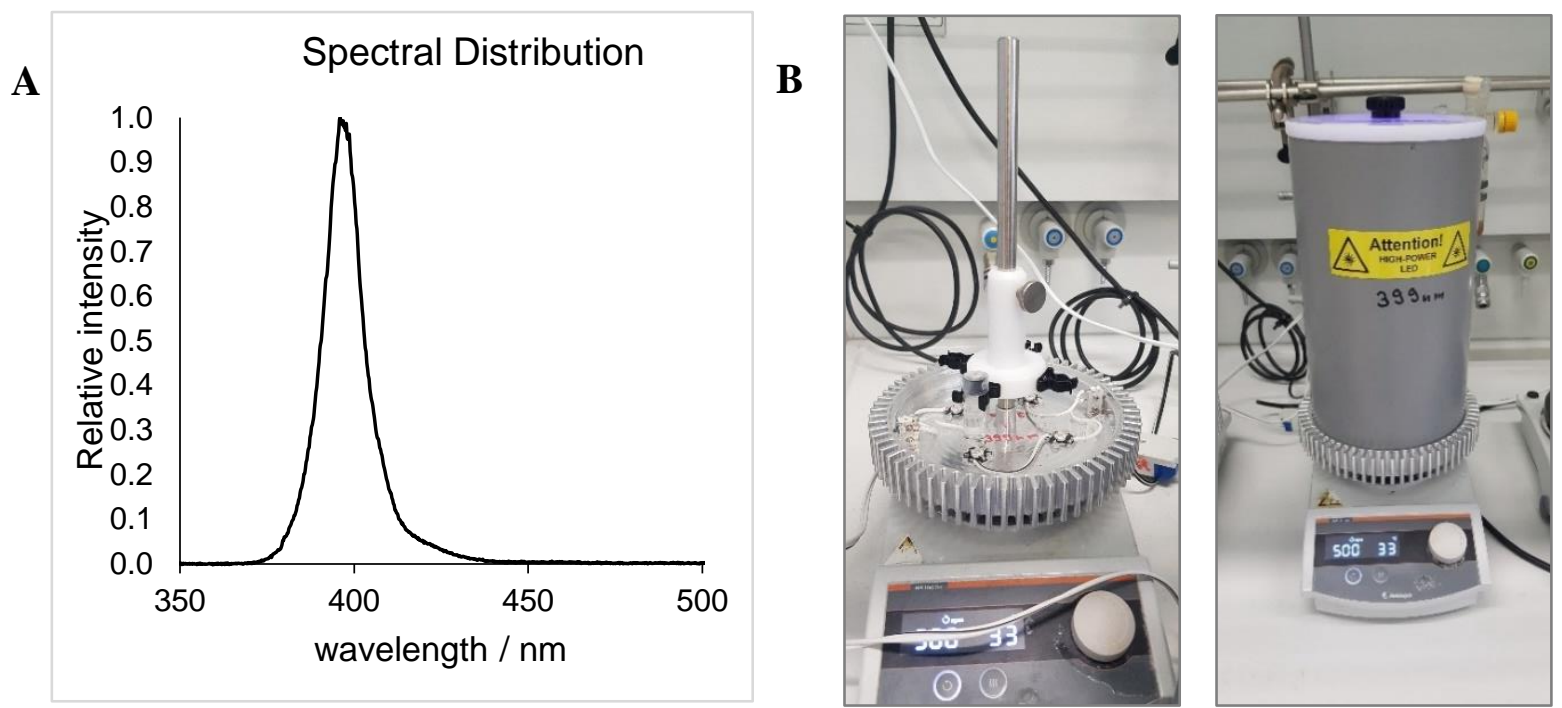

Figure S1: A Emission spectrum of LED lamp: $400 \mathrm{~nm}$; B General set-up for irradiation experiments at r.t..

A
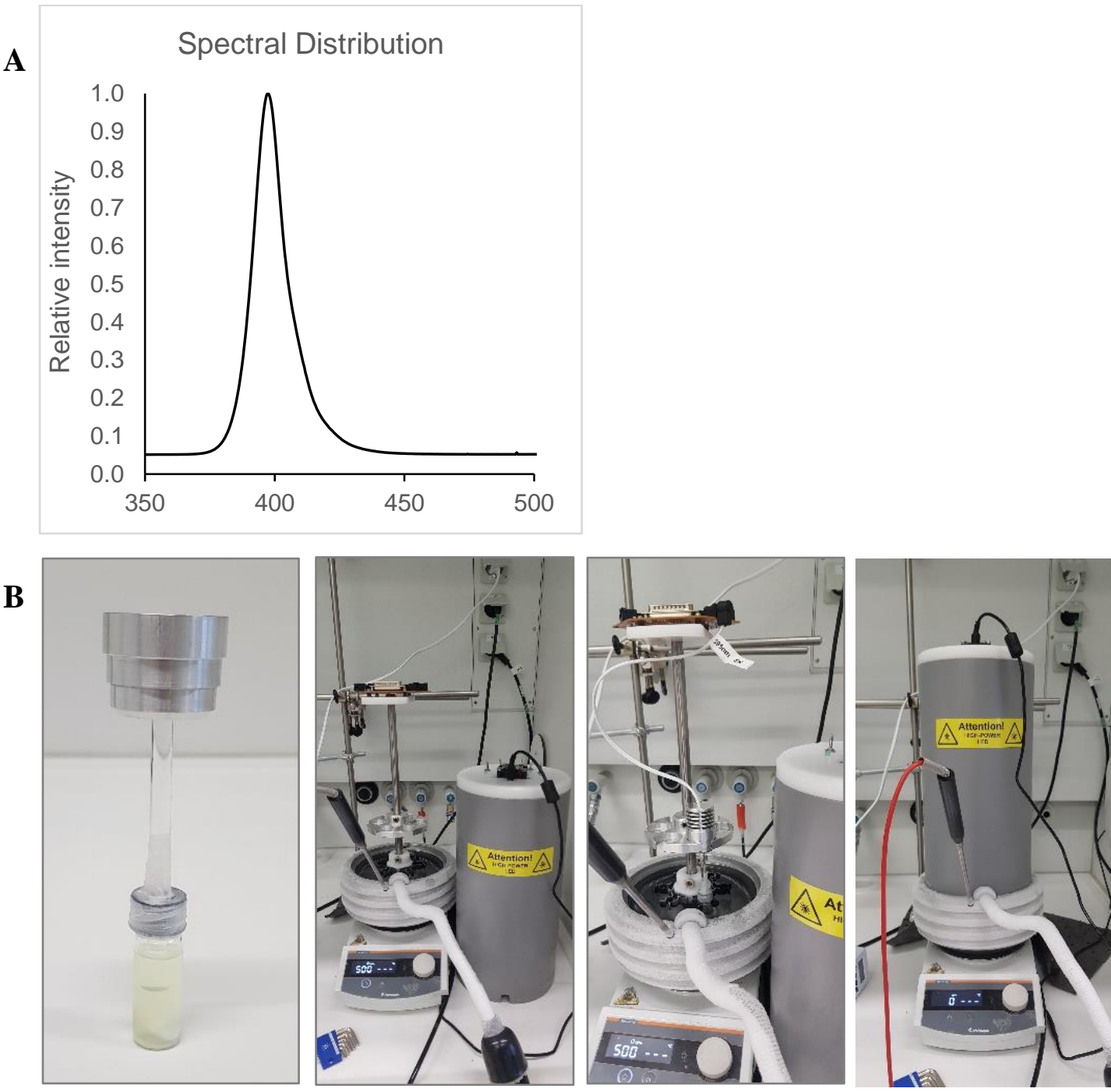

Figure S2: A Emission spectrum of LED lamp: $399 \mathrm{~nm}$; B General set-up for irradiation experiments at low temperature. 


\section{Experimental Section}

\section{Procedures and analytical data}

\section{Synthesis of starting materials}

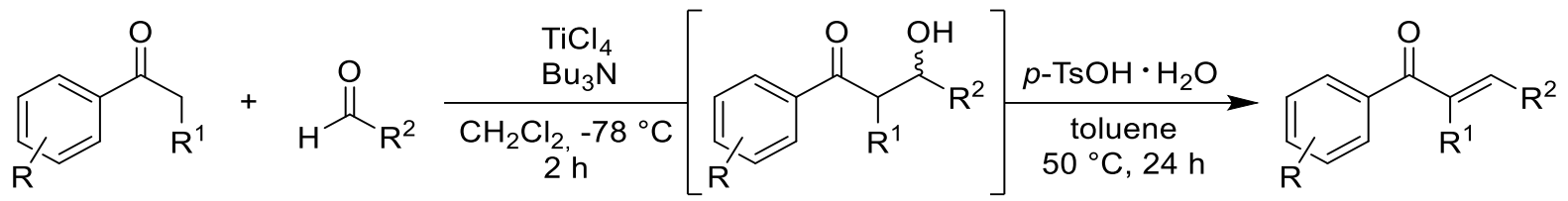

\section{General procedure A for the aldol-addition of acetophenones to enones}

In a flame dried pressure tube under argon the specified ketone $(5.00 \mathrm{mmol}, 1.00$ eq. $)$ was dissolved in dry $\mathrm{CH}_{2} \mathrm{Cl}_{2}(15 \mathrm{~mL})$ and cooled to $-78{ }^{\circ} \mathrm{C}$. $\mathrm{TiCl}_{4}(0.77 \mathrm{~mL}, 6.00 \mathrm{mmol}, 1.20$ eq. $)$ and tributylamine $(1.43 \mathrm{~mL}, 7.00 \mathrm{mmol}, 1.40$ eq.) were added dropwise and the mixture was stirred at $-78{ }^{\circ} \mathrm{C}$ for $30 \mathrm{~min}$. The specified aldehyde $(6.00 \mathrm{mmol}, 1.20 \mathrm{eq}$.) was added and the reaction was stirred at $-78{ }^{\circ} \mathrm{C}$ for $2 \mathrm{~h}$. The reaction was quenched by addition of water $(20 \mathrm{~mL})$ and subsequently warmed to r.t.. The aqueous layer was $3 \mathrm{x}$ extracted with $\mathrm{CH}_{2} \mathrm{Cl}_{2}$ and the combined organic layers were washed with $1 \mathrm{M} \mathrm{HCl}$ (aq.) and dried over $\mathrm{MgSO}_{4}$. In some cases, incomplete conversion was observed by TLC (n-pentane/EtOAc 9:1) and remaining starting material was removed by column filtration $\left(\mathrm{SiO}_{2}, n\right.$-pentane/EtOAc 9:1). The crude aldol product was dissolved in toluene $(50 \mathrm{~mL}), p-\mathrm{TsOH} \cdot \mathrm{H}_{2} \mathrm{O}(190 \mathrm{mg}, 1.00 \mathrm{mmol}, 0.20 \mathrm{eq}$. was added and the mixture was heated at $50{ }^{\circ} \mathrm{C}$ until completion (judged by TLC). After concentration on silica the material was purified by column chromatography $\left(\mathrm{SiO}_{2}\right)$ to yield the specified alkene.

\section{(E)-2,4-Dimethyl-1-phenylpent-2-en-1-one (1)}

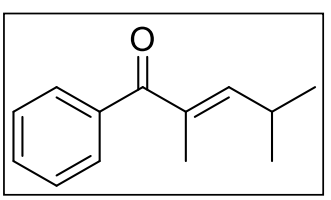

Prepared according to General Procedure A, propiophenone $(0.66 \mathrm{~mL}, \quad 5.00 \mathrm{mmol}, \quad 1.00$ eq. $)$ and isobutyraldehyde $(0.55 \mathrm{~mL}$, $6.00 \mathrm{mmol}, 1.20$ eq.) were converted to 1 . Purification by column chromatography $\left(\mathrm{SiO}_{2}, n\right.$-pentane/EtOAc 98:2) yielded the product as a colorless oil (785 mg, $4.17 \mathrm{mmol}, 83 \%)$.

$\mathrm{R}_{f}=0.75$ (n-pentane/EtOAc 19:1); ${ }^{1} \mathrm{H}$ NMR (400 MHz, $\left.\mathrm{CDCl}_{3}\right): \delta=7.66-7.59(\mathrm{~m}, 2 \mathrm{H}), 7.58$ $-7.43(\mathrm{~m}, 1 \mathrm{H}), 7.45-7.37$ (m, 2H), 6.10 (dq, $J=9.5,1.4 \mathrm{~Hz}, 1 \mathrm{H}), 2.78$ (dhept, $J=9.6,6.7 \mathrm{~Hz}$, 1H), $1.97(\mathrm{~d}, J=1.5 \mathrm{~Hz}, 3 \mathrm{H}), 1.03(\mathrm{~d}, J=6.7 \mathrm{~Hz}, 6 \mathrm{H}) \mathrm{ppm}$; HR-ESI-MS: $m / z: 211.1104$ 
([M+Na $]^{+}$, calcd. for $\mathrm{C}_{13} \mathrm{H}_{16} \mathrm{ONa}^{+}$: 211.1093), $399.2297\left([2 M+\mathrm{Na}]^{+}\right.$, calcd. for $\mathrm{C}_{26} \mathrm{H}_{32} \mathrm{O}_{2} \mathrm{Na}^{+}$: 399.2295); analytical data in agreement with literature. ${ }^{[2]}$

\section{(E)-1-(4-Methoxyphenyl)-2,4-dimethylpent-2-en-1-one (24)}

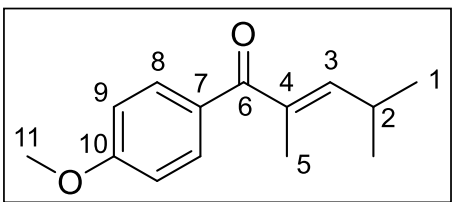

Prepared according to General Procedure A, 4'-methyoxypropiophenone $\quad(0.77 \mathrm{~mL}, \quad 5.00 \mathrm{mmol}, \quad 1.00$ eq. $) \quad$ and isobutyraldehyde $(0.55 \mathrm{~mL}, 6.00 \mathrm{mmol}, 1.20$ eq. $)$ were converted to 24. Purification by column chromatography ( $\mathrm{SiO}_{2}, n$-pentane/EtOAc 98:2) yielded the product as a yellow oil (877 $\mathrm{mg}, 4.02 \mathrm{mmol}, 81 \%)$.

$\mathrm{R}_{f}=0.39$ (n-pentane/EtOAc 19:1); ${ }^{1} \mathrm{H}$ NMR $\left(500 \mathrm{MHz}, \mathrm{CDCl}_{3}\right): \delta=7.71-7.64(\mathrm{~m}, 2 \mathrm{H}, \mathrm{H} 8)$, $6.93-6.89$ (m, 2H, H9), 6.01 (dq, $J=9.5,1.4 \mathrm{~Hz}, 1 \mathrm{H}, \mathrm{H} 3$ ), 3.86 (s, 3H, H11), 2.76 (dhept, $J=9.5,6.6 \mathrm{~Hz}, 1 \mathrm{H}, \mathrm{H} 2), 1.96(\mathrm{~d}, J=1.4 \mathrm{~Hz}, 3 \mathrm{H}, \mathrm{H} 5), 1.04(\mathrm{~d}, J=6.7 \mathrm{~Hz}, 6 \mathrm{H}, \mathrm{H} 1) \mathrm{ppm} ;{ }^{13} \mathrm{C}$ NMR (126 MHz, $\mathrm{CDCl}_{3}$ ): $\delta=198.4$ (C6), 162.6 (C10), 151.1 (C3), 134.0 (C4), 131.9 (C8), 131.1 (C7), 113.4 (C9), 55.5 (C11), 28.4 (C2), 22.2 (C1), 12.9 (C5) ppm; IR (ATR): $\tilde{v}=$ 2959(m), 2869(w), 1639(s), 1600(s), 1574(m), 1507(m), 1463(m), 1417(w), 1386(w), 1362(w), 1307(m), 1252(s), 1164(s), 1108(w), 1022(m), 968(w), 895(w), 839(m), 799(w), 760(m), 699(m) $\mathrm{cm}^{-1}$; HR-ESI-MS: $\mathrm{m} / z: 241.11944\left([M+\mathrm{Na}]^{+}\right.$, calcd. for $\mathrm{C}_{14} \mathrm{H}_{18} \mathrm{O}_{2} \mathrm{Na}^{+}:$241.11990).

\section{(E)-2,4-Dimethyl-1-(p-tolyl)pent-2-en-1-one (25)}

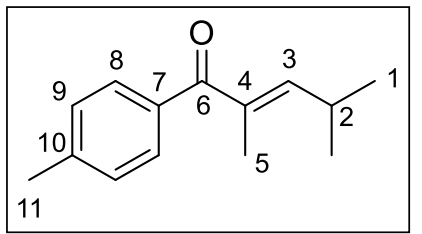

Prepared according to General Procedure A, 4'-methylpropiophenone $\quad(0.75 \mathrm{~mL}, \quad 5.00 \mathrm{mmol}, \quad 1.00$ eq. $) \quad$ and isobutyraldehyde $(0.55 \mathrm{~mL}, 6.00 \mathrm{mmol}, 1.20 \mathrm{eq}$.) were converted to 25. Purification by column chromatography $\left(\mathrm{SiO}_{2}, n\right.$-pentane/EtOAc 99:1) yielded the product as a pale-yellow oil (720 mg, $3.56 \mathrm{mmol}, 71 \%)$.

$\mathrm{R}_{f}=0.85$ (n-pentane/EtOAc 9:1); ${ }^{1} \mathrm{H} \mathrm{NMR}\left(600 \mathrm{MHz}, \mathrm{CDCl}_{3}\right): \delta=7.56(\mathrm{~m}, 2 \mathrm{H}, \mathrm{H} 8), 7.22(\mathrm{~m}$, 2H, H9), 6.07 (dq, $J=9.6,1.3 \mathrm{~Hz}, 1 \mathrm{H}, \mathrm{H} 3$ ), 2.77 (dhept, $J=9.5,6.7 \mathrm{~Hz}, 1 \mathrm{H}, \mathrm{H} 2), 2.40$ (s, 3H, H11), 1.97 (s, 3H, H5), 1.03 (d, $J=6.7 \mathrm{~Hz}, 6 \mathrm{H}, \mathrm{H} 1) \mathrm{ppm} ;{ }^{13} \mathrm{C}$ NMR (151 MHz, $\mathrm{CDCl}_{3}$ ): $\delta=199.2$ (C6), 152.4 (C3), 142.1 (C10), 136.0 (C7), 134.1 (C4), 129.8 (C8), 128.8 (C9), 28.5 (C2), 22.1 (C1), 21.6 (C11), 12.7 (C5) ppm; IR (ATR): $\tilde{v}=$ 2960(w), 2928(w), 2869(w), 1645(s), 1607(m), 1569(w), 1463(w), 1387(w), 1362(w), 1313(m), 1270(s), 1174(m), 1104(w), 1028(m), 1015(m), 968(w), 895(w), 829(m), 797(w), 746(s), 714(w), 695(m) cm ${ }^{-1}$; HR-ESIMS: $m / z: 225.12474\left([M+\mathrm{Na}]^{+}\right.$, calcd. for $\left.\mathrm{C}_{14} \mathrm{H}_{18} \mathrm{ONa}^{+}: 225.12499\right)$. 


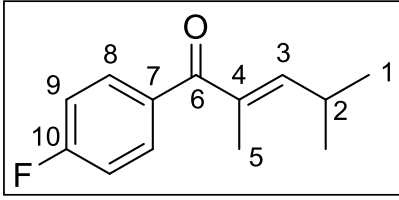

Prepared according to General Procedure A, 4'-fluoropropiophenone $(0.69 \mathrm{~mL}, 5.00 \mathrm{mmol}, 1.00 \mathrm{eq}$.) and isobutyraldehyde ( $0.55 \mathrm{~mL}, 6.00 \mathrm{mmol}, 1.20$ eq.) were converted to 26. Purification by column chromatography $\left(\mathrm{SiO}_{2}, n\right.$-pentane/EtOAc 98:2) yielded the product as a pale-yellow oil (772 mg, $3.74 \mathrm{mmol}, 75 \%)$.

$\mathrm{R}_{f}=0.67$ (n-pentane/EtOAc 19:1); ${ }^{1} \mathrm{H}$ NMR (600 MHz, $\left.\mathrm{CDCl}_{3}\right): \delta=7.69-7.63(\mathrm{~m}, 2 \mathrm{H}, \mathrm{H} 8)$, 7.09 (m, 2H, H9), 6.04 (dq, $J=9.5,1.3 \mathrm{~Hz}, 1 \mathrm{H}, \mathrm{H} 3$ ), 2.77 (dp, $J=9.3,6.6 \mathrm{~Hz}, 1 \mathrm{H}, \mathrm{H} 2), 1.97$ 1.95 (m, 3H, H5), 1.04 (d, $J=6.7 \mathrm{~Hz}, 6 \mathrm{H}, \mathrm{H1}) \mathrm{ppm} ;{ }^{13} \mathrm{C} \mathrm{NMR}\left(151 \mathrm{MHz}, \mathrm{CDCl}_{3}\right): \delta=198.0$ (C6), 164.9 (d, J = 252.3 Hz, C10), 152.9 (C3), 134.9 (d, $J=3.2 \mathrm{~Hz}, \mathrm{C} 7), 134.1$ (C4), 132.0 (d, $J=8.8 \mathrm{~Hz}, \mathrm{C} 8) 115.3(\mathrm{~d}, J=21.7 \mathrm{~Hz}, \mathrm{C} 9), 28.6$ (C2), 22.1 (C1), 12.6 (C5) ppm; ${ }^{19} \mathrm{~F}$ NMR (564 $\mathrm{MHz}, \mathrm{CDCl}_{3}$ ) $\delta=-108.0$ (s) ppm; IR (ATR): $\tilde{v}=2962(\mathrm{w}), 2929(\mathrm{w}), 2870(\mathrm{w}), 1646(\mathrm{~s}), 1599(\mathrm{~s})$, 1504(m), 1465(w), 1407(w), 1364(w), 1311(m), 1270(m), 1226(s), 1183(w), 1155(m), 1095(w), 1024(m), 1013(m), 969(w), 896(w), 848(m), 816(m), 756(s), 693(m) cm ${ }^{-1}$; HR-ESIMS: $m / z: 207.11761\left([M+\mathrm{H}]^{+}\right.$, calcd. for $\mathrm{C}_{13} \mathrm{H}_{15} \mathrm{FOH}^{+}:$207.11797).

\section{(E)-1-(4-Bromophenyl)-2,4-dimethylpent-2-en-1-one (27)}

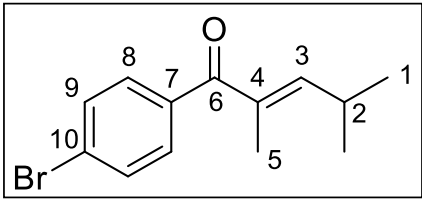

Prepared according to General Procedure A, 4'-bromopropiophenone ( $1.07 \mathrm{~g}, 5.00 \mathrm{mmol}, 1.00 \mathrm{eq}$.) and isobutyraldehyde (0.55 mL, $6.00 \mathrm{mmol}, 1.20$ eq.) were converted to 27. Purification by column chromatography $\left(\mathrm{SiO}_{2}, n\right.$-pentane/EtOAc 98:2) yielded the product as a pale-yellow oil (1.12 g, $4.19 \mathrm{mmol}, 84 \%)$.

$\mathrm{R}_{f}=0.58$ (n-pentane/EtOAc 19:1); ${ }^{1} \mathrm{H}$ NMR (600 MHz, $\left.\mathrm{CDCl}_{3}\right): \delta=7.57-7.53(\mathrm{~m}, 2 \mathrm{H}, \mathrm{H} 9)$, $7.52-7.46$ (m, 2H, H8), 6.05 (dq, $J=9.5,1.3 \mathrm{~Hz}, 1 \mathrm{H}, \mathrm{H} 3$ ), 2.77 (dhept, $J=9.3,6.7 \mathrm{~Hz}, 1 \mathrm{H}$, $\mathrm{H} 2), 1.96$ (s, 3H, H5), 1.03 (d, $J=6.7 \mathrm{~Hz}, 6 \mathrm{H}, \mathrm{H} 1) \mathrm{ppm} ;{ }^{13} \mathrm{C} \mathrm{NMR}\left(151 \mathrm{MHz}, \mathrm{CDCl}_{3}\right): \delta=198.2$ (C6), 153.6 (C3), 137.6 (C7), 134.1 (C4), 131.4 (C9), 131.1 (C8), 126.3 (C10), 28.6 (C2), 22.1 (C1), 12.5 (C5) ppm; IR (ATR): $\tilde{v}=$ 2960(w), 2927(w), 2869(w), 1647(s), 1585(s), 1481(w), 1464(w), 1393(m), 1363(w), 1311(m), 1266(m), 1171(w), 1098(w), 1069(m), 1024(m), 1009(s), 969(w), 895(m), 834(m), 747(s), 692(w), 671(w) cm ${ }^{-1}$; HR-EI-MS: $m / z: 266.03039$ $\left([M]^{+}\right.$, calcd. for $\left.\mathrm{C}_{13} \mathrm{H}_{15} \mathrm{BrO}^{+}: 266.03008\right) ; 268.02843\left([M]^{+}\right.$, calcd. for $\mathrm{C}_{13} \mathrm{H}_{15} \mathrm{BrO}^{+}$: 268.02803). 


\section{(E)-1-(3-Chlorophenyl)-2,4-dimethylpent-2-en-1-one (28)}

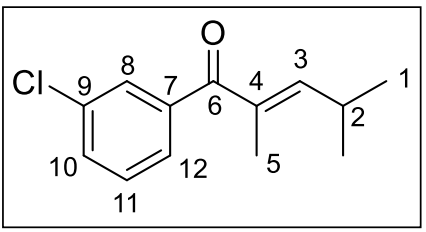

Prepared according to General Procedure A, 3'-chloropropiophenone (843 $\mathrm{mg}, \quad 5.00 \mathrm{mmol}, \quad 1.00$ eq. $) \quad$ and isobutyraldehyde $(0.55 \mathrm{~mL}, 6.00 \mathrm{mmol}, 1.20$ eq.) were converted to

28. Purification by column chromatography $\left(\mathrm{SiO}_{2}, \mathrm{CyH} / \mathrm{EtOAc}\right.$ 99:1) yielded the product as a pale-yellow oil (366 mg, $1.64 \mathrm{mmol}, 33 \%)$.

$\mathrm{R}_{f}=0.74$ (n-pentane/EtOAc 19:1); ${ }^{1} \mathrm{H}$ NMR $\left(600 \mathrm{MHz}, \mathrm{CDCl}_{3}\right): \delta=7.59(\mathrm{~m}, 1 \mathrm{H}, \mathrm{H} 8), 7.47$ (m, 2H, H10/H12), 7.35 (m, 1H, H11), $6.12-6.06$ (m, 1H, H3), 2.78 (dhept, $J=9.6,6.7$ Hz, 1H, H2), 1.96 (dd, $J=1.4,0.7 \mathrm{~Hz}, 3 \mathrm{H}, \mathrm{H} 5), 1.04$ (dd, $J=6.7,0.8 \mathrm{~Hz}, 6 \mathrm{H}, \mathrm{H} 1) \mathrm{ppm} ;{ }^{13} \mathrm{C} \mathrm{NMR}$ (151 MHz, $\mathrm{CDCl}_{3}$ ): $\delta=197.8$ (C6), 154.1 (C3), 140.6 (C7), 134.4 (C9), 134.1 (C4), 131.4 (C10/C12), 129.5 (C8/C11), 129.5 (C8/C11), 127.5 (C10/C12), 28.7 (C2), 22.1 (C1), 12.4 (C5) ppm; IR (ATR): $\tilde{v}=$ 2961(w), 2869(w), 1651(s), 1568(m), 1465(m), 1415(w), 1388(w), 1363(w), 1311(m), 1268(m), 1182(m), 1078(w), 1027(m), 973(w), 893(w), 801(w), 773(m), 739(s), 694(m) cm$~_{-1}$; HR-ESI-MS: $m / z: 223.08839\left([M+\mathrm{H}]^{+}\right.$, calcd. for $\mathrm{C}_{13} \mathrm{H}_{15} \mathrm{ClOH}^{+}$: 223.08842).

\section{(E)-2-Methyl-1,4-diphenylbut-2-en-1-one (29)}

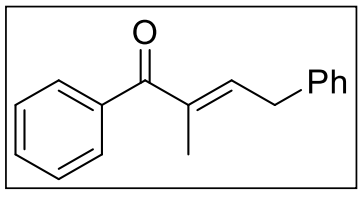

Prepared according to General Procedure A, propiophenone (0.66 mL, $5.00 \mathrm{mmol}, 1.00$ eq.) and phenylacetaldehyde $(0.68 \mathrm{~mL}$, $6.00 \mathrm{mmol}, 1.20$ eq.) were converted to 29. Purification by column chromatography $\left(\mathrm{SiO}_{2}, n\right.$-pentane/EtOAc 99:1) yielded the product as a colorless oil (695 mg, $2.94 \mathrm{mmol}, 59 \%)$.

$\mathrm{R}_{f}=0.37$ (n-pentane/EtOAc 19:1); ${ }^{1} \mathrm{H}$ NMR (400 MHz, $\left.\mathrm{CDCl}_{3}\right): \delta=7.68-7.61(\mathrm{~m}, 2 \mathrm{H}), 7.53$ - $7.44(\mathrm{~m}, 1 \mathrm{H}), 7.44-7.35(\mathrm{~m}, 2 \mathrm{H}), 7.35-7.27$ (m, 2H), 7.25 - $7.21(\mathrm{~m}, 1 \mathrm{H}), 7.21$ - 7.14 (m, 2H), 6.47 (tq, $J=7.4,1.4 \mathrm{~Hz}, 1 \mathrm{H}), 3.65$ (d, $J=7.4 \mathrm{~Hz}, 2 \mathrm{H}), 2.11(\mathrm{~s}, 3 \mathrm{H}) \mathrm{ppm}$; HR-ESI-MS: $m / z: 259.10910\left([M+N a]^{+}\right.$, calcd. for $\left.\mathrm{C}_{17} \mathrm{H}_{26} \mathrm{ONa}^{+}: 259.10933\right)$; analytical data in agreement with literature. ${ }^{[3]}$ 


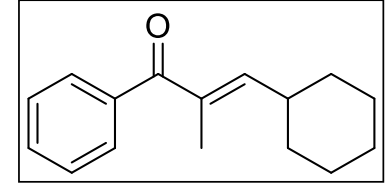

Prepared according to General Procedure A, propiophenone $(0.66 \mathrm{~mL}, \quad 5.00 \mathrm{mmol}, \quad 1.00 \mathrm{eq}$.$) and cyclohexanecarboxaldehyde$ (0.73 mL, $6.00 \mathrm{mmol}, 1.20$ eq.) were converted to 22. Purification by column chromatography $\left(\mathrm{SiO}_{2}, \mathrm{CH}_{2} \mathrm{Cl}_{2}\right)$ yielded the product as a colorless oil $(586 \mathrm{mg}$, $2.56 \mathrm{mmol}, 51 \%)$.

$\mathrm{R}_{f}=0.37\left(\mathrm{CH}_{2} \mathrm{Cl}_{2}\right) ;{ }^{1} \mathrm{H} \mathrm{NMR}\left(400 \mathrm{MHz}, \mathrm{CDCl}_{3}\right): \delta=7.64-7.59(\mathrm{~m}, 2 \mathrm{H}), 7.53-7.46(\mathrm{~m}, 1 \mathrm{H})$, $7.44-7.37(\mathrm{~m}, 2 \mathrm{H}), 6.11(\mathrm{dq}, J=9.5,1.4 \mathrm{~Hz}, 1 \mathrm{H}), 2.53-2.39(\mathrm{~m}, 1 \mathrm{H}), 1.98(\mathrm{~d}, J=1.4 \mathrm{~Hz}$, 3H), $1.77-1.64(\mathrm{~m}, 5 \mathrm{H}), 1.41-1.26(\mathrm{~m}, 2 \mathrm{H}), 1.24-1.03(\mathrm{~m}, 3 \mathrm{H}) \mathrm{ppm}$; HR-ESI-MS: $m / z$ : $251.14034\left([M+\mathrm{Na}]^{+}\right.$, calcd. for $\left.\mathrm{C}_{16} \mathrm{H}_{20} \mathrm{ONa}^{+}: 251.14063\right)$; analytical data in agreement with literature. $^{[4]}$

\section{(E)-3-Cyclopentyl-2-methyl-1-phenylprop-2-en-1-one (30)}

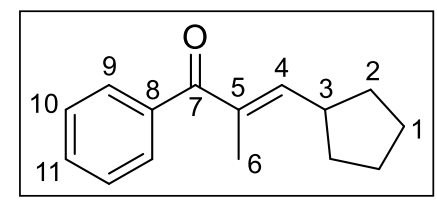

Prepared according to General Procedure A, propiophenone (0.66 mL, $5.00 \mathrm{mmol}, 1.00$ eq.) and cyclopentanecarboxaldehyde ( $0.64 \mathrm{~mL}, 6.00 \mathrm{mmol}, 1.20$ eq.) were converted to 30. Purification by column chromatography $\left(\mathrm{SiO}_{2}, \mathrm{CH}_{2} \mathrm{Cl}_{2}\right)$ yielded the product as a colorless oil $(661 \mathrm{mg}$, $3.08 \mathrm{mmol}, 62 \%)$.

$\mathrm{R}_{f}=0.25\left(\mathrm{CH}_{2} \mathrm{Cl}_{2}\right) ;{ }^{1} \mathrm{H}$ NMR $\left(600 \mathrm{MHz}, \mathrm{CDCl}_{3}\right): \delta=7.64-7.60(\mathrm{~m}, 2 \mathrm{H}, \mathrm{H} 9), 7.52-7.43(\mathrm{~m}$, 1H, H11), $7.43-7.38$ (m, 2H, H10), 6.22 (dd, $J=9.4,1.5 \mathrm{~Hz}, 1 \mathrm{H}, \mathrm{H} 4), 2.87$ (h, $J=8.4 \mathrm{~Hz}$, 1H, H3), 1.99 (d, J = 1.4 Hz, 3H, H6), 1.90 (m, 2H, H2), 1.74 - 1.57 (m, 4H, H1), 1.33 - 1.24 (m, 2H, H2') ppm; ${ }^{13} \mathrm{C} \mathrm{NMR} \mathrm{(151} \mathrm{MHz,} \mathrm{CDCl}_{3}$ ): $\delta=199.3$ (C7), 152.2 (C4), 139.0 (C8), 135.0 (C5), 131.4 (C11), 129.5 (C9), 128.1 (C10), 40.1 (C3), 33.2 (C2), 25.7 (C1), 12.7 (C6) ppm; IR (ATR): $\tilde{v}=2951(\mathrm{~m}), 2867(\mathrm{w}), 1643(\mathrm{~s}), 1597(\mathrm{w}), 1577(\mathrm{w}), 1446(\mathrm{~m}), 1384(\mathrm{w}), 1365(\mathrm{w})$, 1333(w), 1287(m), 1261(m), 1191(w), 1176(w), 1132(w), 1108(w), 1075(w), 1008(s), 933(w), 905(m), 789(w), 709(s) cm ${ }^{-1}$; HR-ESI-MS: m/z: $237.12486\left([M+\mathrm{Na}]^{+}\right.$, calcd. for $\mathrm{C}_{15} \mathrm{H}_{18} \mathrm{ONa}^{+}$: 237.12498). 


\section{(E)-3-Cyclopropyl-2-methyl-1-phenylprop-2-en-1-one (31)}

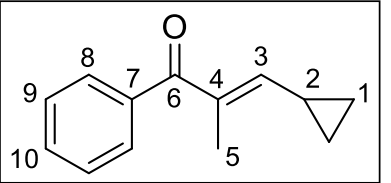

Prepared according to General Procedure A, propiophenone $(0.66 \mathrm{~mL}, 5.00 \mathrm{mmol}, \quad 1.00 \mathrm{eq}$.$) and cyclopropylcarboxaldehyde$ ( $0.45 \mathrm{~mL}, 6.00 \mathrm{mmol}, 1.20$ eq.) were converted to 31. Purification by column chromatography $\left(\mathrm{SiO}_{2}, \mathrm{CH}_{2} \mathrm{Cl}_{2}\right)$ yielded the product as a white solid $(273 \mathrm{mg}$, $1.47 \mathrm{mmol}, 29 \%)$.

$\mathrm{R}_{f}=0.59\left(\mathrm{CH}_{2} \mathrm{Cl}_{2}\right) ;{ }^{1} \mathrm{H}$ NMR $\left(600 \mathrm{MHz}, \mathrm{CDCl}_{3}\right): \delta=7.58-7.54(\mathrm{~m}, 2 \mathrm{H}, \mathrm{H} 8), 7.48-7.44(\mathrm{~m}$, 1H, H10), 7.41 - 7.35 (m, 2H, H9), 5.67 (d, J=10.3 Hz, 1H, H3), 2.08 (s, 3H, H5), 1.75 (dddd, $J=12.5,9.6,8.0,4.5 \mathrm{~Hz}, 1 \mathrm{H}, \mathrm{H} 2), 1.03-0.95$ (m, 2H, H1), $0.55-0.50$ (m, 2H, H1') ppm; ${ }^{13} \mathrm{C}$ NMR (151 MHz, $\mathrm{CDCl}_{3}$ ): $\delta=198.4$ (C6), 152.8 (C3), 139.3 (C7), 134.5 (C4), 131.1 (C10), 129.2 (C8), 128.1 (C9), 12.6 (C5), 12.3 (C2), 8.9 (C1) ppm; IR (ATR): $\tilde{v}=3066(\mathrm{w}), 3006(\mathrm{w})$, 2921(w), 1625(s), 1597(m), 1575(m), 1444(m), 1425(w), 1391(m), 1372(w), 1331(m), 1306(w), 1276(s), 1204(m), 1193(m), 1179(m), 1111(w), 1077(w), 1053(m), 1035(m), 1007(m), $966(\mathrm{~s}), 933(\mathrm{w}), 923(\mathrm{w}), 895(\mathrm{~s}), 861(\mathrm{~m}), 806(\mathrm{~m}), 787(\mathrm{~m}), 738(\mathrm{w}), 710(\mathrm{~s}), 697(\mathrm{~s}) \mathrm{cm}^{-}$ 1, HR-ESI-MS: $m / z: 209.09352\left([M+N a]^{+}\right.$, calcd. for $\left.\mathrm{C}_{13} \mathrm{H}_{14} \mathrm{ONa}^{+}: 209.09368\right), \mathrm{Mp}=45.3-$ $47.1^{\circ} \mathrm{C}$.

\section{(E)-1-(4-Trifluoromethyl)-2,4-dimethylpent-2-en-1-one (32)}

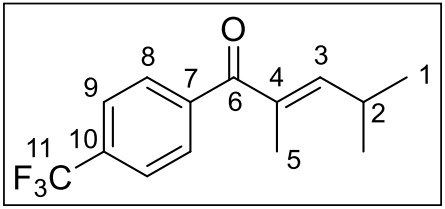

Prepared according to General Procedure A, 4'trifluoromethyl-propiophenone $(1.01 \mathrm{~g}, 5.00 \mathrm{mmol}, 1.00 \mathrm{eq}$.$) and$ isobutyraldehyde $(0.55 \mathrm{~mL}, 6.00 \mathrm{mmol}, 1.20 \mathrm{eq}$.) were converted

to 32. Purification by column chromatography $\left(\mathrm{SiO}_{2}, n\right.$-pentane/EtOAc $\left.98: 2\right)$ yielded the product as a colorless oil (971 $\mathrm{mg}, 3.78 \mathrm{mmol}, 76 \%)$.

$\mathrm{R}_{f}=0.52$ (n-pentane/EtOAc 19:1); ${ }^{1} \mathrm{H}$ NMR $\left(500 \mathrm{MHz}, \mathrm{CDCl}_{3}\right): \delta=7.68$ (m, 4H, H8/H9), 6.08 (dq, $J=9.5,1.3 \mathrm{~Hz}, 1 \mathrm{H}, \mathrm{H} 3$ ), 2.79 (dhept, $J=9.4,6.6 \mathrm{~Hz}, 1 \mathrm{H}, \mathrm{H} 2$ ), 1.98 (d, $J=1.4 \mathrm{~Hz}, 3 \mathrm{H}$, H5), 1.03 (d, $J=6.7 \mathrm{~Hz}, 6 \mathrm{H}, \mathrm{H1}$ ) ppm; ${ }^{13} \mathrm{C} \mathrm{NMR}\left(126 \mathrm{MHz}, \mathrm{CDCl}_{3}\right): \delta=198.1$ (C6), 154.9 (C3), 142.3 (q, $J=1.3 \mathrm{~Hz}, \mathrm{C} 7), 134.3$ (C4), 132.9 (q, $J=32.7 \mathrm{~Hz}, \mathrm{C} 10), 129.6$ (C8), 125.2 (q, $J=3.8 \mathrm{~Hz}, \mathrm{C} 9), 123.9$ (q, $J=272.5 \mathrm{~Hz}, \mathrm{C} 11), 28.7$ (C2), 22.0 (C1), 12.2 (C5) ppm; ${ }^{19} \mathrm{~F}$ NMR $\left(470 \mathrm{MHz}, \mathrm{CDCl}_{3}\right) \delta=-63.0$ (s) ppm; IR (ATR): $\tilde{v}=2965(\mathrm{w}), 1652(\mathrm{~m}), 1509(\mathrm{w}), 1466(\mathrm{w})$, 1408(w), 1323(s), 1314(s), 1271(m), 1165(m), 1125(s), 1106(s), 1065(s), 1041(m), 1025(w), 971(w), 851(m), 781(w), 766(m), 728(m), 708(m), 667(m) $\mathrm{cm}^{-1}$; HR-ESI-MS: $\mathrm{m} / z: 257.11459$ 
$\left([M+\mathrm{H}]^{+}\right.$, calcd. for $\mathrm{C}_{14} \mathrm{H}_{15} \mathrm{~F}_{3} \mathrm{OH}^{+}$: 257.11477); $237.10829\left([M-\mathrm{F}]^{+}\right.$, calcd. for $\mathrm{C}_{14} \mathrm{H}_{15} \mathrm{~F}_{2} \mathrm{O}^{+}$: 237.10855).

(E)-6-Methoxy-2-(2-methylpropylidene)-3,4-dihydronaphthalen-1(2H)-one (33)

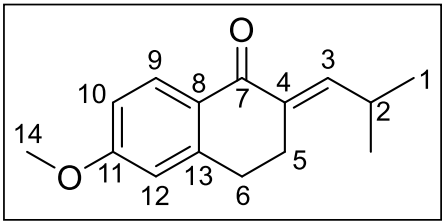

Prepared according to General Procedure A, 7-methoxy-4chromanone ( $881 \mathrm{mg}, 5.00 \mathrm{mmol}, 1.00$ eq.) and isobutyraldehyde (0.55 mL, $6.00 \mathrm{mmol}, 1.20$ eq.) were converted to 33. Purification by column chromatography $\left(\mathrm{SiO}_{2}, n\right.$-pentane/EtOAc 9:1) yielded the product as an off-white solid (978 $\mathrm{mg}, 4.24 \mathrm{mmol}, 85 \%)$.

$\mathrm{R}_{f}=0.50\left(n\right.$-pentane/EtOAc 9:1); ${ }^{1} \mathrm{H}$ NMR $\left(500 \mathrm{MHz}, \mathrm{CDCl}_{3}\right): \delta=8.06(\mathrm{~d}, J=8.7 \mathrm{~Hz}, 1 \mathrm{H}$, H9), 6.83 (dd, $J=8.7,2.6 \mathrm{~Hz}, 1 \mathrm{H}, \mathrm{H} 10), 6.71$ (dt, $J=9.8,1.7 \mathrm{~Hz}, 1 \mathrm{H}, \mathrm{H} 3), 6.68$ (d, $J=2.5 \mathrm{~Hz}$, 1H, H12), 3.85 (s, 3H, H14), $2.94-2.87$ (m, 2H, H6), 2.80 - 2.74 (m, 2H, H5), 2.70 (dhept, $J=9.7,6.6 \mathrm{~Hz}, 1 \mathrm{H}, \mathrm{H} 2), 1.07$ (d, $J=6.7 \mathrm{~Hz}, 6 \mathrm{H}, \mathrm{H} 1) \mathrm{ppm} ;{ }^{13} \mathrm{C} \mathrm{NMR}\left(126 \mathrm{MHz}, \mathrm{CDCl}_{3}\right)$ : $\delta=187.0(\mathrm{C} 7), 163.5$ (C11), 146.2 (C13), 145.8 (C3), 133.1 (C4), 130.7 (C9), 127.3 (C8), 113.2 (C10), 112.5 (C12), 55.5 (C14), 29.8 (C6), 27.5 (C2), 25.9 (C5), 22.5 (C1) ppm; IR (ATR): $\tilde{v}$ $=2959(\mathrm{w}), 2868(\mathrm{w}), 2840(\mathrm{w}), 1670(\mathrm{~m}), 1619(\mathrm{~s}), 1493(\mathrm{w}), 1463(\mathrm{w}), 1445(\mathrm{w}), 1363(\mathrm{w})$, 1304(s), 1255(s), 1220(s), 1154(m), 1126(m), 1118(m), 1088(m), 1034(m), 1020(m), 914(m), 872(w), 849(w), 820(w), 763(m), 728(w), 716(w), 691(w), 653(w) $\mathrm{cm}^{-1}$; HR-ESI-MS: m/z: $253.11939\left([M+\mathrm{Na}]^{+}\right.$, calcd. for $\left.\mathrm{C}_{15} \mathrm{H}_{18} \mathrm{O}_{2} \mathrm{Na}^{+}: 253.11990\right), \mathrm{Mp}=61.2-62.0{ }^{\circ} \mathrm{C}$.

\section{(E)-2-(2-Methylpropylidene)-3,4-dihydronaphthalen-1(2H)-one (23)}

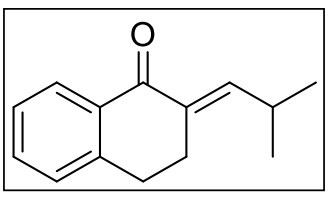

Prepared according to General Procedure A, 1-tetralone $(0.67 \mathrm{~mL}$, $5.00 \mathrm{mmol}, \quad 1.00$ eq.) and isobutyraldehyde $(0.55 \mathrm{~mL}, 6.00 \mathrm{mmol}$, 1.20 eq.) were converted to $\mathbf{2 3}$. Note: the condensation occurred during the work-up of the aldol addition and heating in the presence of $p$-TsOH was not necessary. Purification by column chromatography $\left(\mathrm{SiO}_{2}, n\right.$-pentane/EtOAc 98:2 $\rightarrow$ 97:3) yielded the product as a yellow oil (724 $\mathrm{mg}, 3.61 \mathrm{mmol}, 72 \%)$.

$\mathrm{R}_{f}=0.48$ (n-pentane/EtOAc 19:1); ${ }^{1} \mathrm{H}$ NMR (400 MHz, $\left.\mathrm{CDCl}_{3}\right): \delta=8.09$ (dd, $J=7.8,1.5 \mathrm{~Hz}$, $1 \mathrm{H}), 7.45(\mathrm{td}, J=7.4,1.5 \mathrm{~Hz}, 1 \mathrm{H}), 7.32(\mathrm{td}, J=7.4,1.2 \mathrm{~Hz}, 1 \mathrm{H}), 7.25-7.22(\mathrm{~m}, 1 \mathrm{H}), 6.76(\mathrm{dt}$, $J=9.8,1.7 \mathrm{~Hz}, 1 \mathrm{H}), 2.99-2.91(\mathrm{~m}, 2 \mathrm{H}), 2.83-2.77(\mathrm{~m}, 2 \mathrm{H}), 2.72(\mathrm{dp}, J=9.8,6.6 \mathrm{~Hz}, 1 \mathrm{H})$, $1.08(\mathrm{~d}, J=6.7 \mathrm{~Hz}, 6 \mathrm{H})$ ppm; HR-ESI-MS: $m / z: 223.10894\left([M+\mathrm{Na}]^{+}\right.$, calcd. for $\mathrm{C}_{14} \mathrm{H}_{16} \mathrm{ONa}^{+}$: 223.10933); analytical data in agreement with literature. ${ }^{[5]}$ 


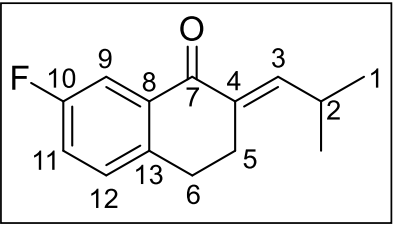

Prepared according to General Procedure A, 7-fluoro-1-tetralone (821 mg, $5.00 \mathrm{mmol}, 1.00$ eq.) and isobutyraldehyde $(0.55 \mathrm{~mL}$, $6.00 \mathrm{mmol}, 1.20$ eq.) were converted to 34. Purification by column chromatography $\left(\mathrm{SiO}_{2}, n\right.$-pentane/EtOAc $\left.98: 2 \rightarrow 95: 5\right)$ yielded the product as a colorless oil (943 $\mathrm{mg}, 4.32 \mathrm{mmol}, 86 \%)$.

$\mathrm{R}_{f}=0.62\left(n\right.$-pentane/EtOAc 9:1); ${ }^{1} \mathrm{H}$ NMR (500 MHz, $\left.\mathrm{CDCl}_{3}\right): \delta=7.74(\mathrm{dd}, J=9.2,2.8 \mathrm{~Hz}$, 1H, H9), $7.23-7.19$ (m, 1H, H12), 7.15 (td, $J=8.3,2.8$ Hz, 1H, H11), 6.77 (dt, $J=9.8,1.7$ Hz, 1H, H3), $2.93-2.89$ (m, 2H, H6), $2.80-2.76$ (m, 2H, H5), 2.71 (dhept, $J=9.8,6.7$ Hz, 1H, $\mathrm{H} 2), 1.08(\mathrm{~d}, J=6.6 \mathrm{~Hz}, 6 \mathrm{H}, \mathrm{H} 1) \mathrm{ppm} ;{ }^{13} \mathrm{C} \mathrm{NMR}\left(126 \mathrm{MHz}, \mathrm{CDCl}_{3}\right): \delta=187.0(\mathrm{~d}, J=2.1 \mathrm{~Hz}$, C7), 161.9 (d, $J=245.8 \mathrm{~Hz}, \mathrm{C} 10), 147.5$ (C3), 139.5 (d, $J=3.0 \mathrm{~Hz}, \mathrm{C} 13), 135.4$ (d, $J=6.3 \mathrm{~Hz}$, C8), 132.4 (d, $J=1.2 \mathrm{~Hz}, \mathrm{C} 4), 130.1$ (d, $J=7.1 \mathrm{~Hz}, \mathrm{C} 12), 120.3$ (d, $J=22.1 \mathrm{~Hz}, \mathrm{C} 11), 114.2$ (d, $J=22.0 \mathrm{~Hz}, \mathrm{C} 9), 28.6$ (C6), 27.6 (C2), 25.7 (C5), 22.4 (C1) ppm; ${ }^{19} \mathrm{~F}$ NMR (470 MHz, $\left.\mathrm{CDCl}_{3}\right): \delta=-115.1(\mathrm{dddt}, J=9.4,7.9,5.2,1.3 \mathrm{~Hz}) \mathrm{ppm}$; IR (ATR): $\tilde{v}=2962(\mathrm{~m}), 2869(\mathrm{w})$, 1676(w), 1623(m), 1606(s), 1587(m), 1491(s), 1465(w), 1424(s), 1384(w), 1366(w), 1306(s), 1270(s), 1242(s), 1193(w), 1165(m), 1120(w), 1099(w), 1078(w), 1027(w), 1013(w), 945(w), 899(s), 823(m), 808(m), 766(s), 737(s), 690(m) cm ${ }^{-1}$; HR-ESI-MS: m/z: $241.09976\left([M+\mathrm{Na}]^{+}\right.$, calcd. for $\mathrm{C}_{14} \mathrm{H}_{15} \mathrm{FONa}^{+}$: 241.09991).

\section{(E)-7-Bromo-2-(2-methylpropylidene)-3,4-dihydronaphthalen-1(2H)-one (35)}

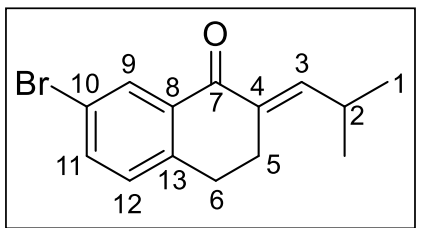

Prepared according to General Procedure A, 7-bromo-1tetralone $(1.13 \mathrm{~g}, 5.00 \mathrm{mmol}, 1.00 \mathrm{eq}$.$) and isobutyraldehyde$ (0.55 mL, $6.00 \mathrm{mmol}, 1.20$ eq.) were converted to 35. Purification by column chromatography $\left(\mathrm{SiO}_{2}, n\right.$-pentane/EtOAc 98:2 $\rightarrow$ 95:5) yielded the product as a white solid $(1.21 \mathrm{~g}, 4.33 \mathrm{mmol}, 87 \%)$.

$\mathrm{R}_{f}=0.69\left(n\right.$-pentane/EtOAc 9:1); ${ }^{1} \mathrm{H}$ NMR $\left(600 \mathrm{MHz}, \mathrm{CDCl}_{3}\right): \delta=8.19(\mathrm{~d}, J=2.2 \mathrm{~Hz}, 1 \mathrm{H}$, H9), 7.55 (dd, $J=8.1,2.2 \mathrm{~Hz}, 1 \mathrm{H}, \mathrm{H} 11), 7.12$ (d, $J=8.1 \mathrm{~Hz}, 1 \mathrm{H}, \mathrm{H} 12), 6.77$ (dt, $J=9.9,1.7 \mathrm{~Hz}$, 1H, H3), $2.91-2.86$ (m, 2H, H6), $2.79-2.76$ (m, 2H, H5), 2.71 (dhept, $J=9.8,6.6 \mathrm{~Hz}, 1 \mathrm{H}$, $\mathrm{H} 2), 1.08$ (d, $J=6.6 \mathrm{~Hz}, 6 \mathrm{H}, \mathrm{H} 1) \mathrm{ppm} ;{ }^{13} \mathrm{C} \mathrm{NMR}\left(151 \mathrm{MHz}, \mathrm{CDCl}_{3}\right): \delta=186.7$ (C7), 147.7 (C3), 142.5 (C13), 135.8 (C11), 135.3 (C8), 132.3 (C4), 131.0 (C9), 130.2 (C12), 121.0 (C10), 28.8 (C6), 27.6 (C2), 25.5 (C5), 22.4 (C1) ppm; IR (ATR): $\tilde{v}=$ 2959(m), 2937(w), 2867(w), 1672(s), 1629(s), 1584(m), 1461(m), 1431(w), 1402(m), 1384(w), 1314(m), 1301(m), 1263(m), 
1236(m), 1210(s), 1176(w), 1154(w), 1127(m), 1102(w), 1089(w), 1071(w), 1023(m), 1010(w), 957(w), 934(m), 895(m), 878(m), 828(s), 794(m), 759(s), 707(m), 655(m) cm ${ }^{-1}$; HRESI-MS: $m / z$ : $301.01986\left([M+N a]^{+}\right.$, calcd. for $\mathrm{C}_{14} \mathrm{H}_{15} \mathrm{BrONa}^{+}$: 301.01985$)$; 303.01765 $\left([M+\mathrm{Na}]^{+}\right.$, calcd. for $\left.\mathrm{C}_{14} \mathrm{H}_{15} \mathrm{BrONa}^{+}: 303.01780\right) ; \mathrm{Mp}=49.2-49.9^{\circ} \mathrm{C}$.

\section{(E)-5,7-Dimethyl-2-(2-methylpropylidene)-3,4-dihydronaphthalen-1(2H)-one (36)}

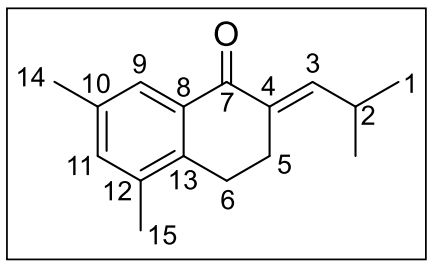

Prepared according to General Procedure A, 5,7-dimethyl-1tetralone ( $871 \mathrm{mg}, 5.00 \mathrm{mmol}, 1.00$ eq.) and isobutyraldehyde ( $0.55 \mathrm{~mL}, 6.00 \mathrm{mmol}, 1.20$ eq.) were converted to 36 . Purification by column chromatography $\left(\mathrm{SiO}_{2}, n\right.$-pentane/EtOAc 98:2 $\rightarrow$ 95:5) yielded the product as a yellow oil $(1.00 \mathrm{~g}, 4.38 \mathrm{mmol}, 88 \%)$.

$\mathrm{R}_{f}=0.62\left(n\right.$-pentane/EtOAc 9:1); ${ }^{1} \mathrm{H}$ NMR $\left(600 \mathrm{MHz}, \mathrm{CDCl}_{3}\right): \delta=7.82-7.78(\mathrm{~m}, 1 \mathrm{H}, \mathrm{H} 9)$, $7.19-7.15$ (m, 1H, H11), 6.69 (dt, $J=9.8,1.7 \mathrm{~Hz}, 1 \mathrm{H}, \mathrm{H} 3), 2.86-2.81$ (m, 2H, H6), $2.78-$ 2.75 (m, 2H, H5), 2.72 (dhept, $J=9.8,6.7$ Hz, 1H, H2), 2.34 (s, 3H, H14), 2.29 (s, 3H, H15), $1.08(\mathrm{~d}, J=6.7 \mathrm{~Hz}, 6 \mathrm{H}, \mathrm{H} 1) \mathrm{ppm} ;{ }^{13} \mathrm{C} \mathrm{NMR}\left(151 \mathrm{MHz}, \mathrm{CDCl}_{3}\right): \delta=188.7$ (C7), $145.8(\mathrm{C} 3)$, 139.4 (C13), 136.0 (C10), 135.7 (C11), 135.6 (C8), 133.8 (C12), 133.1 (C4), 126.3 (C9), 27.5 (C2), 25.6 (C6), 25.5 (C5), 22.5 (C1), 21.0 (C14), 19.5 (C15) ppm; IR (ATR): $\tilde{v}=2959(\mathrm{~m})$, 2868(w), 1673(s), 1623(m), 1606(s), 1475(m), 1446(w), 1409(w), 1381(w), 1362(w), 1311(s), 1284(m), 1261(w), 1224(w), 1196(s), 1164(s), 1121(w), 1099(w), 1043(w), 1013(w), 969(w), 933(m), 893(s), 901(w), 764(s), 737(m), 695(w), 665(m) cm² ; HR-ESI-MS: m/z: 251.14014 $\left([M+\mathrm{Na}]^{+}\right.$, calcd. for $\left.\mathrm{C}_{16} \mathrm{H}_{20} \mathrm{ONa}^{+}: 251.14063\right)$.

\section{(E)-3-(2-Methylpropylidene)chroman-4-one (37)}

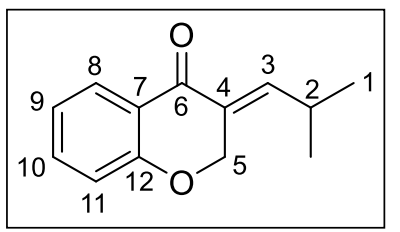

Prepared according to General Procedure A, 4-chromanone (741 mg, $5.00 \mathrm{mmol}, \quad 1.00$ eq.) and isobutyraldehyde $(0.55 \mathrm{~mL}$, $6.00 \mathrm{mmol}, 1.20$ eq.) were converted to 37. Purification by column chromatography $\left(\mathrm{SiO}_{2}, n\right.$-pentane/EtOAc $\left.97: 3\right)$ yielded the product as a colorless oil (730 mg, $3.61 \mathrm{mmol}, 72 \% ; E: Z=89: 11$ as determined by $\left.{ }^{1} \mathrm{H}-\mathrm{NMR}\right)$.

$\mathrm{R}_{f}=0.72$ (n-pentane/EtOAc 9:1); E-isomer: ${ }^{1} \mathrm{H}$ NMR (500 MHz, $\left.\mathrm{CDCl}_{3}\right): \delta=7.98$ (ddd, $J=7.9,1.8,0.5 \mathrm{~Hz}, 1 \mathrm{H}, \mathrm{H} 8$ ), 7.45 (ddd, $J=8.3,7.2,1.8 \mathrm{~Hz}, 1 \mathrm{H}, \mathrm{H} 10$ ), 7.03 (ddd, $J=7.9,7.2$, $1.1 \mathrm{~Hz}, 1 \mathrm{H}, \mathrm{H} 9), 6.95$ (ddd, $J=8.4,1.1,0.5 \mathrm{~Hz}, 1 \mathrm{H}, \mathrm{H} 11), 6.75$ (dt, $J=10.2,1.7 \mathrm{~Hz}, 1 \mathrm{H}, \mathrm{H} 3$ ), $5.04(\mathrm{~d}, J=1.7 \mathrm{~Hz}, 2 \mathrm{H}, \mathrm{H} 5), 2.66$ (dhept, $J=10.3,6.6 \mathrm{~Hz}, 1 \mathrm{H}, \mathrm{H} 2), 1.09$ (d, $J=6.6 \mathrm{~Hz}, 6 \mathrm{H}$, 
H1) ppm; ${ }^{13} \mathrm{C}$ NMR (126 MHz, $\left.\mathrm{CDCl}_{3}\right): \delta=182.6$ (C6), 161.7 (C12), 147.4 (C3), 135.7 (C10), 128.8 (C4), 128.1 (C8), 122.3 (C7), 121.9 (C9), 118.0 (C11), 66.9 (C5), 27.5 (C2), 22.3 (C1) ppm; Z-isomer: ${ }^{1} \mathrm{H}$ NMR (500 MHz, $\left.\mathrm{CDCl}_{3}\right): \delta=7.98-7.93(\mathrm{~m}, 1 \mathrm{H}, \mathrm{H} 8), 7.46-7.42(\mathrm{~m}, 1 \mathrm{H}$, H10), 7.02 (ddd, $J=8.1,7.1,1.1 \mathrm{~Hz}, 1 \mathrm{H}, \mathrm{H} 9), 6.94$ (ddd, $J=8.3,1.1,0.4 \mathrm{~Hz}, 1 \mathrm{H}, \mathrm{H} 11$ ), 5.94 (dt, $J=9.8,1.3 \mathrm{~Hz}, 1 \mathrm{H}, \mathrm{H} 3), 4.82$ (d, $J=1.3 \mathrm{~Hz}, 2 \mathrm{H}, \mathrm{H} 5), 3.58$ (dhept, $J=9.8,6.6 \mathrm{~Hz}, 1 \mathrm{H}$, $\mathrm{H} 2), 1.06$ (d, $J=6.7 \mathrm{~Hz}, 6 \mathrm{H}, \mathrm{H} 1) ;{ }^{13} \mathrm{C} \mathrm{NMR}\left(126 \mathrm{MHz}, \mathrm{CDCl}_{3}\right)$ : $\delta=184.2$ (C6), $161.2(\mathrm{C} 12)$, 152.2 (C3), 135.6 (C10), 127.9 (C8), 127.8 (C4), 123.1 (C7), 121.7 (C9), 117.9 (C11), 73.8 (C5), 28.2 (C2), 22.5 (C1); HR-ESI-MS: m/z: $225.08843\left([M+\mathrm{Na}]^{+}\right.$, calcd. for $\mathrm{C}_{13} \mathrm{H}_{14} \mathrm{O}_{2} \mathrm{Na}^{+}$: 225.08860).

\section{(E)-2-(2-Methylpropylidene)-2,3-dihydro-1H-inden-1-one (38)}

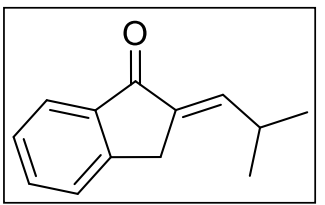

Prepared according to a modified General Procedure A, 1-indanone (661 mg, $5.00 \mathrm{mmol}, \quad 1.00$ eq.) and isobutyraldehyde $(0.55 \mathrm{~mL}$, $6.00 \mathrm{mmol}, 1.20 \mathrm{eq}$.) were converted to 38. Note: the condensation occurred during the aldol addition and heating in the presence of $p$-TsOH was not necessary. Purification by column chromatography $\left(\mathrm{SiO}_{2}, n\right.$-pentane/EtOAc $98: 2$ to $\left.97: 3\right)$ yielded the product as a yellow sticky solid (655 mg, $3.52 \mathrm{mmol}, 70 \%)$.

$\mathrm{R}_{f}=0.37$ (n-pentane/EtOAc 19:1); ${ }^{1} \mathrm{H}$ NMR $\left(400 \mathrm{MHz}, \mathrm{CDCl}_{3}\right): \delta=7.85(\mathrm{~d}, J=7.7 \mathrm{~Hz}, 1 \mathrm{H})$, $7.58(\mathrm{td}, J=7.4,1.3 \mathrm{~Hz}, 1 \mathrm{H}), 7.49(\mathrm{dt}, J=7.6,1.0 \mathrm{~Hz}, 1 \mathrm{H}), 7.38(\mathrm{td}, J=7.4,1.0 \mathrm{~Hz}, 1 \mathrm{H}), 6.72$ $(\mathrm{dt}, J=9.9,2.1 \mathrm{~Hz}, 1 \mathrm{H}), 3.68(\mathrm{~d}, J=1.3 \mathrm{~Hz}, 1 \mathrm{H}), 2.66(\mathrm{dhept}, J=9.9,6.7 \mathrm{~Hz}, 1 \mathrm{H}), 1.12(\mathrm{~d}$, $J=6.7 \mathrm{~Hz}, 6 \mathrm{H})$ ppm; HR-ESI-MS: $m / z: 209.09338\left([M+\mathrm{Na}]^{+}\right.$, calcd. for $\mathrm{C}_{13} \mathrm{H}_{14} \mathrm{ONa}^{+}$: 209.09368); analytical data in agreement with literature. ${ }^{[6]}$

\section{(E)-6-(2-Methylpropylidene)-6,7,8,9-tetrahydro-5H-benzo[7]annulen-5-one (39)}

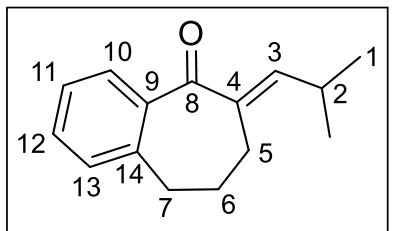

Prepared according to General Procedure A, 1-benzosuberone $(0.75 \mathrm{~mL}, 5.00 \mathrm{mmol}, 1.00$ eq. $)$ and isobutyraldehyde $(0.55 \mathrm{~mL}$, $6.00 \mathrm{mmol}, 1.20$ eq.) were converted to 39. Purification by column chromatography $\left(\mathrm{SiO}_{2}, n\right.$-pentane/EtOAc $\left.97: 3\right)$ yielded the product as a white solid (519 mg, $2.42 \mathrm{mmol}, 48 \%$ ).

$\mathrm{R}_{f}=0.59$ (n-pentane/EtOAc 19:1); ${ }^{1} \mathrm{H}$ NMR (500 MHz, $\left.\mathrm{CDCl}_{3}\right): \delta=7.71(\mathrm{dd}, J=7.6,1.5 \mathrm{~Hz}$, 1H, H10), 7.41 (td, $J=7.5,1.5 \mathrm{~Hz}, 1 \mathrm{H}, \mathrm{H} 12), 7.31$ (td, $J=7.5,1.3 \mathrm{~Hz}, 1 \mathrm{H}, \mathrm{H} 11), 7.14$ (ddd, $J$ $=7.5,1.3,0.6 \mathrm{~Hz}, 1 \mathrm{H}, \mathrm{H} 13), 6.68(\mathrm{~d}, J=10.2 \mathrm{~Hz}, 1 \mathrm{H}, \mathrm{H} 3), 2.77$ (t, $J=6.9 \mathrm{~Hz}, 2 \mathrm{H}, \mathrm{H} 7), 2.68$ 
(dhept, $J=10.2,6.6 \mathrm{~Hz}, 1 \mathrm{H}, \mathrm{H} 2), 2.33$ (t, $J=6.9 \mathrm{~Hz}, 2 \mathrm{H}, \mathrm{H} 5), 1.86$ (p, $J=6.9 \mathrm{~Hz}, 2 \mathrm{H}, \mathrm{H} 6$ ), 1.09 (d, $J=6.6 \mathrm{~Hz}, 6 \mathrm{H}, \mathrm{H1}) \mathrm{ppm} ;{ }^{13} \mathrm{C} \mathrm{NMR}\left(126 \mathrm{MHz}, \mathrm{CDCl}_{3}\right): \delta=198.3$ (C8), 147.5 (C3), 139.7 (C14), 139.0 (C9), 135.9 (C4), 132.2 (C12), 129.1 (C13), 129.0 (C10), 127.0 (C11), 31.4 (C7), 27.7 (C2), 26.5 (C6), 24.1 (C5), 22.7 (C1) ppm; IR (ATR): $\tilde{v}=$ 2965(m), 2947(w), 2925(w), 2859(w), 1669(s), 1615(s), 1594(s), 1457(m), 1381(w), 1360(w), 1300(s), 1249(s), 1192(w), 1101(m), 960(s), 901(s), 877(m), 830(m), 796(w), 765(s), 723(m), 712(s) cm ${ }^{-1}$; HREI-MS: $m / z: 214.13530\left([M]^{+}\right.$, calcd. for $\left.\mathrm{C}_{15} \mathrm{H}_{18} \mathrm{O}^{+}: 214.13522\right) ; \mathrm{Mp}=54.6-55 .{ }^{\circ} \mathrm{C}$.

\section{(E)-6-(2-Ethylpropylidene)-6,7,8,9-tetrahydro-5H-benzo[7]annulen-5-one (40)}

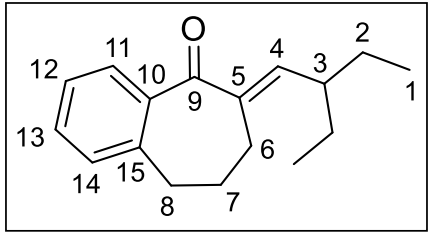

Prepared according to General Procedure A, 1-benzosuberone (0.75 mL, $5.00 \mathrm{mmol}, \quad 1.00$ eq.) and 2-ethylbutyraldehyde (0.74 mL, $6.00 \mathrm{mmol}, 1.20$ eq.) were converted to 40. Purification by column chromatography $\left(\mathrm{SiO}_{2}, n\right.$-pentane/EtOAc 98:2 $\rightarrow$ 95:5) yielded the product as a colorless oil $(1.00 \mathrm{~g}, 4.13 \mathrm{mmol}, 83 \%)$.

$\mathrm{R}_{f}=0.66\left(n\right.$-pentane/EtOAc 9:1); ${ }^{1} \mathrm{H}$ NMR (600 MHz, $\left.\mathrm{CDCl}_{3}\right): \delta=7.73$ (dd, $J=7.6,1.5 \mathrm{~Hz}$, 1H, H11), 7.43 (td, $J=7.5,1.4 \mathrm{~Hz}, 1 \mathrm{H}, \mathrm{H} 13), 7.33$ (td, $J=7.5,1.2 \mathrm{~Hz}, 1 \mathrm{H}, \mathrm{H} 12), 7.15$ (dd, $J=$ 7.5, 1.2 Hz, 1H, H14), 6.61 (d, $J=10.9 \mathrm{~Hz}, 1 \mathrm{H}, \mathrm{H} 4), 2.79$ (t, $J=6.9 \mathrm{~Hz}, 2 \mathrm{H}, \mathrm{H} 8), 2.34$ (t, $J=$ $6.8 \mathrm{~Hz}, 2 \mathrm{H}, \mathrm{H6}$ ), 2.23 (dtt, $J=10.7,9.0,4.9 \mathrm{~Hz}, 1 \mathrm{H}, \mathrm{H} 3$ ), 1.87 (p, $J=6.9 \mathrm{~Hz}, 2 \mathrm{H}, \mathrm{H} 7$ ), 1.57 (dqd, $J=13.5,7.5,4.8 \mathrm{~Hz}, 2 \mathrm{H}, \mathrm{H} 2), 1.38$ (ddq, $J=13.4,8.8,7.4 \mathrm{~Hz}, 2 \mathrm{H}, \mathrm{H} 2$ '), 0.88 (t, $J=7.5$ $\mathrm{Hz}, 6 \mathrm{H}, \mathrm{H1}$ ) ppm; ${ }^{13} \mathrm{C} \mathrm{NMR}\left(151 \mathrm{MHz}, \mathrm{CDCl}_{3}\right.$ ): $\delta=198.0$ (C9), 145.9 (C4), 139.7 (C15), 139.0 (C10), 138.4 (C5), 132.3 (C13), 129.2 (C11), 129.1 (C14), 127.0 (C12), 42.2 (C3), 31.4 (C8), 28.1 (C2), 26.5 (C7), 24.2 (C6), 12.3 (C1) ppm; IR (ATR): $\tilde{v}=$ 2958(m), 2930(m), 2859(w), 1671(s), 1618(s), 1598(m), 1453(s), 1376(w), 1343(w), 1295(s), 1270(m), 1241(s), 1202(w), 1186(w), 1161(w), 1144(w), 1100(m), 1042(w), 995(w), 964(s), 938(w), 907(w), 877(w), 858(w), 822(w), 793(w), 762(s), 709(s) $\mathrm{cm}^{-1}$; HR-ESI-MS: m/z: $265.15586\left([M+\mathrm{Na}]^{+}\right.$, calcd. for $\mathrm{C}_{17} \mathrm{H}_{22} \mathrm{ONa}^{+}$: 265.15628).

tert-Butyl (E)-4-((5-oxo-5,7,8,9-tetrahydro-6H-benzo[7]annulen-6-ylidene)methyl)piperidine-1-carboxylate (41)

Prepared according to a modified General Procedure A, 1-benzosuberone $(0.75 \mathrm{~mL}$, $5.00 \mathrm{mmol}, 1.00$ eq.) and 1-Boc-piperidine-4-carboxaldehyde (1.28 g, $6.00 \mathrm{mmol}, 1.20 \mathrm{eq}$. 


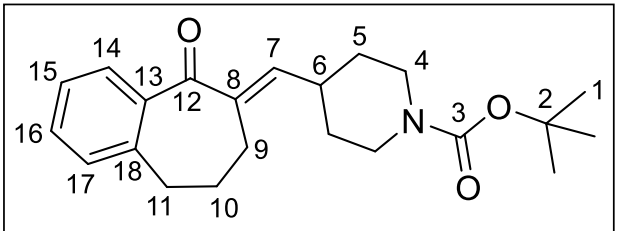

were converted to 41. Note: the condensation was achieved by heating the crude material in toluene in the absence of $p$-TsOH$\cdot \mathrm{H}_{2} \mathrm{O}$. Purification by column chromatography $\left(\mathrm{SiO}_{2}, n\right.$-pentane/EtOAc 9:1) yielded the product as a white solid (1.18 g, $3.32 \mathrm{mmol}, 66 \%)$.

$\mathrm{R}_{f}=0.31$ (n-pentane/EtOAc 9:1); ${ }^{1} \mathrm{H} \mathrm{NMR}\left(600 \mathrm{MHz}, \mathrm{CDCl}_{3}\right): \delta=7.71(\mathrm{dd}, J=7.7,1.4 \mathrm{~Hz}$, 1H, H14), 7.43 (td, $J=7.5,1.5 \mathrm{~Hz}, 1 \mathrm{H}, \mathrm{H} 16), 7.32$ (td, $J=7.6,1.3 \mathrm{~Hz}, 1 \mathrm{H}, \mathrm{H} 15), 7.15$ (dd, $J=$ 7.5, 1.2 Hz, 1H, H17), 6.66 (d, J = 9.9 Hz, 1H, H7), 4.14 (d, J=13.6 Hz, 2H, H4'), 2.84 - 2.75 (m, 4H, H4/H11), 2.50 (dtt, $J=13.8,11.0,3.9 \mathrm{~Hz}, 1 \mathrm{H}, \mathrm{H} 6$ ), 2.36 (t, $J=6.8 \mathrm{~Hz}, 2 \mathrm{H}, \mathrm{H} 9$ ), 1.88 (p, $J=6.9 \mathrm{~Hz}, 2 \mathrm{H}, \mathrm{H} 10), 1.65-1.59$ (m, 2H, H5'), 1.47 (m, 11H, H1/H5) ppm; ${ }^{13} \mathrm{C}$ NMR (151 MHz, $\left.\mathrm{CDCl}_{3}\right): \delta=198.0$ (C12), 154.9 (C3), 143.5 (C7), 139.6 (C18), 138.8 (C13), 137.6 (C8), 132.5 (C16), 129.2 (C14), 129.1 (C17), 127.1 (C15), 79.7 (C2), 43.4 (C4), 35.8 (C6), 31.6 (C5), 31.4 (C11), 28.6 (C1), 26.6 (C10), 24.3 (C9) ppm; IR (ATR): $\tilde{v}=$ 2937(w), 2861(w), 1691(s), 1620(m), 1451(m), 1424(m), 1365(m), 1286(m), 1250(m), 1230(m), 1174(m), 1155(m), 1105(m), 964(m), 914(w), 877(w), 766(m), 710(w) cm-1; HR-ESI-MS: m/z: $378.20374\left([M+\mathrm{Na}]^{+}\right.$, calcd. for $\left.\mathrm{C}_{22} \mathrm{H}_{29} \mathrm{NO}_{3} \mathrm{Na}^{+}: 378.20396\right) ; \mathrm{Mp}=112.7-113.9{ }^{\circ} \mathrm{C}$.

\section{(E)-6-((Tetrahydro-2H-pyran-4-yl)methylene)-6,7,8,9-tetrahydro-5H-benzo[7]annulen-}

\section{5-one (42)}

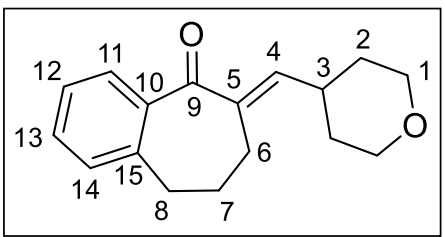

Prepared according to General Procedure A, 1-benzosuberone $(0.75 \mathrm{~mL}, \quad 5.00 \mathrm{mmol}, \quad 1.00 \mathrm{eq}$.$) \quad and tetrahydropyran-4-$ carbaldehyde $(0.62 \mathrm{~mL}, 6.00 \mathrm{mmol}, 1.20$ eq.) were converted to 42. Purification by column chromatography $\left(\mathrm{SiO}_{2}, n\right.$ pentane/EtOAc 9:1) yielded the product as a white solid (1.16 g, $4.53 \mathrm{mmol}, 90 \%)$.

$\mathrm{R}_{f}=0.13\left(n\right.$-pentane/EtOAc 9:1); ${ }^{1} \mathrm{H} \mathrm{NMR}\left(600 \mathrm{MHz}, \mathrm{CDCl}_{3}\right): \delta=7.71(\mathrm{dd}, J=7.6,1.4 \mathrm{~Hz}$, 1H, H11), 7.43 (td, $J=7.5,1.5 \mathrm{~Hz}, 1 \mathrm{H}, \mathrm{H} 13), 7.32$ (td, $J=7.6,1.3 \mathrm{~Hz}, 1 \mathrm{H}, \mathrm{H} 12), 7.15$ (dd, $J=$ 7.6, 1.2 Hz, 1H, H14), 6.68 (d, $J=10.0 \mathrm{~Hz}, 1 \mathrm{H}, \mathrm{H} 4), 4.01$ (ddd, $J=11.5,4.6,2.1 \mathrm{~Hz}, 2 \mathrm{H}, \mathrm{H} 1$ ), $3.48\left(\mathrm{td}, J=11.7,2.3 \mathrm{~Hz}, 2 \mathrm{H}, \mathrm{H} 1^{\prime}\right), 2.78$ (t, $\left.J=6.9 \mathrm{~Hz}, 2 \mathrm{H}, \mathrm{H} 8\right), 2.61$ (tdt, $J=11.2,9.9,4.1 \mathrm{~Hz}$, 1H, H3), 2.36 (t, $J=6.9 \mathrm{~Hz}, 2 \mathrm{H}, \mathrm{H} 6), 1.87$ (p, $J=6.9 \mathrm{~Hz}, 2 \mathrm{H}, \mathrm{H} 7$ ), 1.66 (dtd, $J=13.5,11.6$, $4.4 \mathrm{~Hz}, 2 \mathrm{H}, \mathrm{H} 2), 1.56$ (ddd, $J=13.2,4.3,2.1 \mathrm{~Hz}, 2 \mathrm{H}, \mathrm{H} 2$ ') ppm; ${ }^{13} \mathrm{C} \mathrm{NMR}\left(151 \mathrm{MHz}, \mathrm{CDCl}_{3}\right)$ : $\delta=198.0$ (C9), 143.6 (C4), 139.6 (C15), 138.8 (C10), 137.4 (C5), 132.4 (C13), 129.1 (C11), 129.1 (C14), 127.1 (C12), 67.3 (C1), 34.8 (C3), 32.3 (C2), 31.4 (C8), 26.7 (C7), 24.2 (C6) ppm; IR (ATR): $\tilde{v}=$ 2932(m), 2842(w), 1670(s), 1620(s), 1598(m), 1453(m), 1386(w), 1297(m), 
1266(m), 1252(m), 1237(s), 1185(w), 1162(w), 1123(s), 1103(m), 1083(m), 1042(w), 1013(w), 1000(w), 982(w), 966(s), 930(w), 912(m), 880(m), 861(w), 821(w), 791(w), 765(s), $710(\mathrm{~m}) \mathrm{cm}^{-1}$; HR-ESI-MS: $m / z: 279.13518\left([M+\mathrm{Na}]^{+}\right.$, calcd. for $\mathrm{C}_{17} \mathrm{H}_{20} \mathrm{O}_{2} \mathrm{Na}^{+}:$279.13555); $\mathrm{Mp}$ $=114.8-116.0^{\circ} \mathrm{C}$.

\section{Synthesis of deuterated starting materials}

\section{1-d-Cyclohexanecarboxaldehyde (43)}

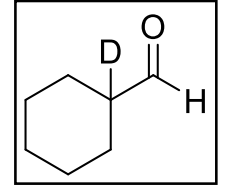

A pressure tube was charged with cyclohexanecarboxaldehyde $(1.21 \mathrm{~mL}$, $10.0 \mathrm{mmol}, 1.00$ eq.) and $\mathrm{K}_{2} \mathrm{CO}_{3}\left(1.38 \mathrm{~g}, 10.0 \mathrm{mmol}, 1.00\right.$ eq.) before $\mathrm{D}_{2} \mathrm{O}$ $(10 \mathrm{~mL})$ was added. The tube was sealed and heated at $70{ }^{\circ} \mathrm{C}$ for $60 \mathrm{~h}$. Afterwards, the mixture was cooled to r.t. and extracted with $\mathrm{CH}_{2} \mathrm{Cl}_{2}(3 \mathrm{x})$. The combined organic layers were dried over $\mathrm{MgSO}_{4}$ and concentrated in vacuo to yield a colorless oil (>95\% D incorporation), which was directly used in the next step without further purification.

${ }^{1} \mathrm{H}$ NMR $\left(400 \mathrm{MHz}, \mathrm{CDCl}_{3}\right): \delta=9.61(\mathrm{~s}, 1 \mathrm{H}), 1.92-1.81(\mathrm{~m}, 2 \mathrm{H}), 1.78-1.68(\mathrm{~m}, 2 \mathrm{H}), 1.68$ $-1.59(\mathrm{~m}, 1 \mathrm{H}), 1.40-1.20(\mathrm{~m}, 5 \mathrm{H}) \mathrm{ppm}$; analytical data in agreement with literature. ${ }^{[7]}$

\section{(E)-3-(Cyclohexyl-1-d)-2-methyl-1-phenylprop-2-en-1-one $(d-22)$}

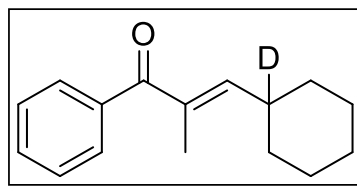

column chromatography $\left(\mathrm{SiO}_{2}, \mathrm{CH}_{2} \mathrm{Cl}_{2}\right)$ yielded the product as a colorless oil (492 $\mathrm{mg}$, $2.15 \mathrm{mmol}, 43 \%,>95 \% \mathrm{D}$ incorporation).

$\mathrm{R}_{f}=0.37\left(\mathrm{CH}_{2} \mathrm{Cl}_{2}\right) ;{ }^{1} \mathrm{H} \mathrm{NMR}\left(500 \mathrm{MHz}, \mathrm{CDCl}_{3}\right): \delta=7.64-7.60(\mathrm{~m}, 2 \mathrm{H}), 7.51-7.47(\mathrm{~m}, 1 \mathrm{H})$, $7.44-7.37(\mathrm{~m}, 2 \mathrm{H}), 6.11(\mathrm{~d}, J=1.4 \mathrm{~Hz}, 1 \mathrm{H}), 1.97(\mathrm{~d}, J=1.4 \mathrm{~Hz}, 3 \mathrm{H}), 1.76-1.65(\mathrm{~m}, 5 \mathrm{H})$, $1.38-1.28(\mathrm{~m}, 2 \mathrm{H}), 1.22-1.15(\mathrm{~m}, 1 \mathrm{H}), 1.10(\mathrm{td}, J=13.3,3.5 \mathrm{~Hz}, 2 \mathrm{H}) \mathrm{ppm} ;{ }^{13} \mathrm{C} \mathrm{NMR}$ $\left(126 \mathrm{MHz}, \mathrm{CDCl}_{3}\right): \delta=199.5,151.8,139.0,134.4,131.4,129.5,128.1,37.9(\mathrm{t}, J=19.0 \mathrm{~Hz})$, 31.9, 26.0, 25.7, 12.6 ppm; HR-ESI-MS: $m / z: 252.14669\left([M+\mathrm{Na}]^{+}\right.$, calcd. for $\mathrm{C}_{16} \mathrm{H}_{19} \mathrm{DONa}^{+}$: 252.14691). 


\section{Catalyst synthesis}

\section{(1S,2R)-cis-1-Dimethylamino-2-indanol (cat. 9)}

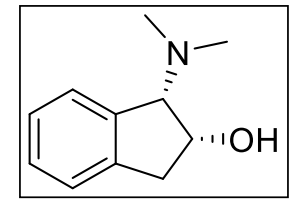

In a pressure tube $(1 S, 2 R)-(-)$-cis-1-amino-2-indanol $(746 \mathrm{mg}$, $5.00 \mathrm{mmol}, 1.00$ eq.) was dissolved in formic acid $(1.89 \mathrm{~mL}, 50.0 \mathrm{mmol}$, 10.0 eq.) and formaldehyde ( $37 \%$ in $\mathrm{H}_{2} \mathrm{O}, 0.45 \mathrm{~mL}, 30.0 \mathrm{mmol}, 6.00$ eq.) was added. The mixture was heated at $100{ }^{\circ} \mathrm{C}$ for $7 \mathrm{~h}$, before being cooled to r.t. and diluted with water. The aqueous layer was washed with $\mathrm{CH}_{2} \mathrm{Cl}_{2}(1 \mathrm{x})$ before being basified $(\mathrm{pH} \sim 14)$ by addition of $1 \mathrm{M} \mathrm{NaOH}$ (aq.). After extraction with $\mathrm{CH}_{2} \mathrm{Cl}_{2}(3 \mathrm{x})$, the combined organic layers were dried over $\mathrm{MgSO}_{4}$ and concentrated in vacuo. Purification by column chromatography $\left(\mathrm{SiO}_{2}, \mathrm{CH}_{2} \mathrm{Cl}_{2} / \mathrm{MeOH}\right.$ 98:2 $\rightarrow$ 9:1) yielded the product as an off-white solid (160 mg, $0.90 \mathrm{mmol}, 18 \%)$.

$\mathrm{R}_{f}=0.41\left(\mathrm{CH}_{2} \mathrm{Cl}_{2} / \mathrm{MeOH} 9: 1\right) ;{ }^{1} \mathrm{H} \mathrm{NMR}\left(400 \mathrm{MHz}, \mathrm{CDCl}_{3}\right): \delta=7.36-7.18(\mathrm{~m}, 4 \mathrm{H}), 4.55(\mathrm{~b}$, $1 \mathrm{H}), 4.50(\mathrm{q}, J=7.9 \mathrm{~Hz}, 1 \mathrm{H}), 4.14(\mathrm{~d}, J=7.8 \mathrm{~Hz}, 1 \mathrm{H}), 3.28(\mathrm{dd}, J=16.4,8.2 \mathrm{~Hz}, 1 \mathrm{H}), 2.83$ $(\mathrm{dd}, J=16.4,7.7 \mathrm{~Hz}, 1 \mathrm{H}), 2.34(\mathrm{~s}, 6 \mathrm{H}) \mathrm{ppm}$; HR-ESI-MS: $m / z: 178.12237\left([M+\mathrm{H}]^{+}\right.$, calcd. for $\mathrm{C}_{11} \mathrm{H}_{15} \mathrm{NOH}^{+}:$178.12264); analytical data in agreement with literature. ${ }^{[8]}$ 
Racemic deconjugative isomerization of alkenes<smiles>[R]C=C([R])C(=O)c1ccc([R])cc1</smiles>

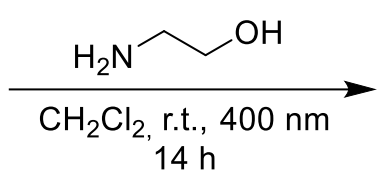<smiles>[R]C=CC([R])C(=O)c1ccccc1</smiles>

General procedure $B$ for the racemic photocatalytic deconjugative isomerization of alkenes

A screw-cap vial with septum under argon was charged with the specified alkene $(0.20 \mathrm{mmol}$, 1.00 eq.) and ethanolamine $(2.4 \mu \mathrm{L}, 0.04 \mathrm{mmol}, 0.20$ eq.). Subsequently, degassed dichloromethane $(4 \mathrm{~mL})$ was added, the vial was sealed and irradiated at $400 \mathrm{~nm}$ for $14 \mathrm{~h}$. Afterwards, the mixture was concentrated on silica and purified by column chromatography $\left(\mathrm{SiO}_{2}, n\right.$-pentane/ $\mathrm{CH}_{2} \mathrm{Cl}_{2}$ ) to yield the products. Note: reactions for scope entries were repeated and yields reported in the paper are the average of two independent experiments.

Optimization of the racemic deconjugative isomerization of alkenes<smiles>CC(=CC(C)C)C(=O)c1ccccc1</smiles>
solvent, r.t., $400 \mathrm{~nm}$ time<smiles>CC(C)=CC(C)C(=O)c1ccccc1</smiles>

Table S1: Optimization of reaction conditions.

\begin{tabular}{ccccccc}
\hline Entry & Solvent & Time [h] & Catalyst & Conc. [mol/L] & Atm. & Yield $^{\text {[a] }}$ \\
\hline 1 & $\mathrm{CH}_{2} \mathrm{Cl}_{2}$ & 14 & - & 0.1 & $\mathrm{Ar}$ & $58 \%$ \\
2 & $\mathrm{CH}_{3} \mathrm{CN}$ & 14 & - & 0.1 & $\mathrm{Ar}$ & $<10 \%$ \\
3 & $\mathrm{CyH}$ & 14 & - & 0.1 & $\mathrm{Ar}$ & $35 \%$ \\
4 & $\mathrm{Toluol}$ & 14 & - & 0.1 & $\mathrm{Ar}$ & $28 \%$ \\
5 & $\mathrm{EtOH}$ & 14 & - & 0.1 & $\mathrm{Ar}$ & $<10 \%$ \\
6 & $\mathrm{THF}$ & 14 & - & 0.1 & $\mathrm{Ar}$ & $<10 \%$ \\
7 & $\mathrm{CH}_{2} \mathrm{Cl}_{2}$ & 14 & - & 0.05 & $\mathrm{Ar}$ & $64 \%$ \\
8 & $\mathrm{CH}_{2} \mathrm{Cl}_{2}$ & 14 & - & 0.2 & $\mathrm{Ar}$ & $60 \%$ \\
9 & $\mathrm{CH}_{2} \mathrm{Cl}_{2}$ & 14 & - & 0.05 & $\mathrm{air}$ & $18 \%$ \\
10 & $\mathrm{CH}_{2} \mathrm{Cl}_{2}$ & 8 & - & 0.05 & $\mathrm{Ar}$ & $67 \%$ \\
11 & $\mathrm{CH}_{2} \mathrm{Cl}_{2}$ & 24 & - & 0.05 & $\mathrm{Ar}$ & $44 \%$ \\
\hline
\end{tabular}

${ }^{[a]}$ All reactions performed on a $0.1 \mathrm{mmol}$ scale. 
<smiles>CC(=CC(C)C)C(=O)c1ccccc1</smiles>

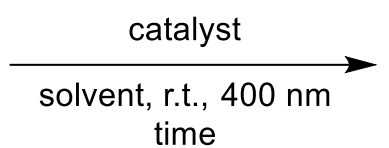<smiles>CC(C)=CC(C)C(=O)c1ccccc1</smiles>

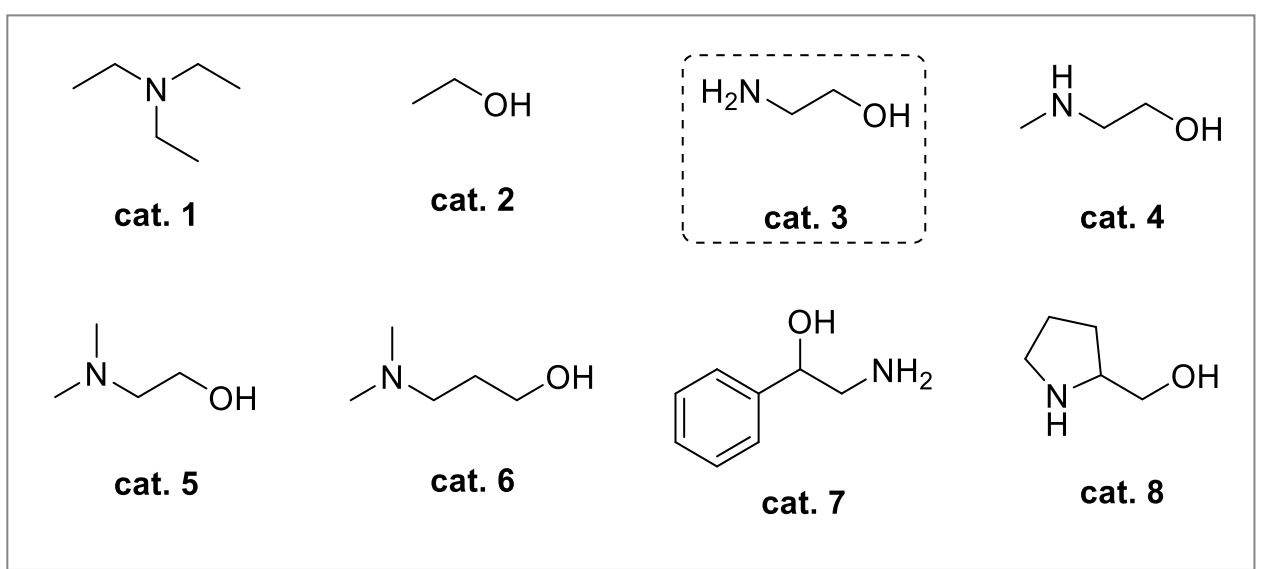

Table S2: Catalyst screen.

\begin{tabular}{cccccccc}
\hline Entry & Solvent & Time [h] & Catalyst & Cat. Loading & Conc. [mol/L] & Atm. & Yield $^{[\mathrm{a}]}$ \\
\hline 12 & $\mathrm{CH}_{2} \mathrm{Cl}_{2}$ & 14 & 1 & $20 \%$ & 0.05 & $\mathrm{Ar}$ & $68 \%$ \\
13 & $\mathrm{CH}_{2} \mathrm{Cl}_{2}$ & 14 & 2 & $20 \%$ & 0.05 & $\mathrm{Ar}$ & $65 \%$ \\
14 & $\mathrm{CH}_{2} \mathrm{Cl}_{2}$ & 14 & 3 & $20 \%$ & 0.05 & $\mathrm{Ar}$ & $\mathbf{7 6 \%}$ \\
15 & $\mathrm{CH}_{2} \mathrm{Cl}_{2}$ & 14 & 4 & $20 \%$ & 0.05 & $\mathrm{Ar}$ & $58 \%$ \\
16 & $\mathrm{CH}_{2} \mathrm{Cl}_{2}$ & 14 & 5 & $20 \%$ & 0.05 & $\mathrm{Ar}$ & $71 \%$ \\
17 & $\mathrm{CH}_{2} \mathrm{Cl}_{2}$ & 14 & 6 & $20 \%$ & 0.05 & $\mathrm{Ar}$ & $52 \%$ \\
18 & $\mathrm{CH}_{2} \mathrm{Cl}_{2}$ & 14 & 7 & $20 \%$ & 0.05 & $\mathrm{Ar}$ & $49 \%$ \\
19 & $\mathrm{CH}_{2} \mathrm{Cl}_{2}$ & 14 & 8 & $20 \%$ & 0.05 & $\mathrm{Ar}$ & $56 \%$ \\
$20^{[b]}$ & $\mathrm{CH}_{2} \mathrm{Cl}_{2}$ & 14 & 3 & $20 \%$ & 0.05 & $\mathrm{Ar}$ & $<5 \%$ \\
21 & $\mathrm{CH}_{2} \mathrm{Cl}_{2}$ & 8 & 3 & $10 \%$ & 0.05 & $\mathrm{Ar}$ & $66 \%$ \\
22 & $\mathrm{CH}_{2} \mathrm{Cl}_{2}$ & 8 & 3 & $20 \%$ & 0.05 & $\mathrm{Ar}$ & $76 \%$ \\
23 & $\mathrm{CH}_{2} \mathrm{Cl}_{2}$ & 8 & 3 & $30 \%$ & 0.05 & $\mathrm{Ar}$ & $73 \%$ \\
\hline
\end{tabular}

${ }^{[a]}$ All reactions performed on a $0.1 \mathrm{mmol} \mathrm{scale} ;{ }^{[b]}$ Reaction performed in the dark.

\section{Product characterization}

\section{2,4-Dimethyl-1-phenylpent-3-en-1-one (( \pm$)-2)$}<smiles>CC=C(C)C=C(C)C(=O)c1ccccc1</smiles>

Prepared according to General Procedure B, 1 (37.7 mg, $0.20 \mathrm{mmol}$, 1.00 eq.) was converted to ( \pm )-2. Purification by column chromatography $\left(\mathrm{SiO}_{2}, n\right.$-pentane/ $\mathrm{CH}_{2} \mathrm{Cl}_{2}$ 7:3) yielded the product as a colorless oil. 
General Procedure B: 1. Reaction: (23.3 mg, $0.12 \mathrm{mmol}, 62 \%)$

2. Reaction: (24.8 mg, $0.13 \mathrm{mmol}, 66 \%)$

$\mathrm{R}_{f}=0.60$ (n-pentane/ $\left.\mathrm{CH}_{2} \mathrm{Cl}_{2} 7: 3\right) ;{ }^{1} \mathrm{H}$ NMR (400 MHz, $\left.\mathrm{CDCl}_{3}\right): \delta=7.98-7.91(\mathrm{~m}, 2 \mathrm{H}), 7.56$ - 7.51 (m, 1H), $7.48-7.40(\mathrm{~m}, 2 \mathrm{H}), 5.20$ (dp, $J=9.5,1.4 \mathrm{~Hz}, 1 \mathrm{H}), 4.27$ (dq, $J=9.5,6.8 \mathrm{~Hz}$, $1 \mathrm{H}), 1.76(\mathrm{~d}, J=1.5 \mathrm{~Hz}, 3 \mathrm{H}), 1.70(\mathrm{~d}, J=1.4 \mathrm{~Hz}, 3 \mathrm{H}), 1.25$ (d, $J=6.8 \mathrm{~Hz}, 3 \mathrm{H})$ ppm; HR-ESIMS: $m / z: 211.10919\left([M+\mathrm{Na}]^{+}\right.$, calcd. for $\left.\mathrm{C}_{13} \mathrm{H}_{16} \mathrm{ONa}^{+}: 211.10933\right)$; analytical data in agreement with literature. ${ }^{[8]}$

\section{1-(4-Methoxyphenyl)-2,4-dimethylpent-3-en-1-one (( \pm$)-3)$}

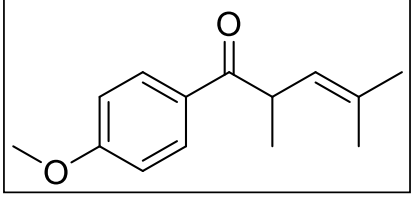

a colorless oil.

Prepared according to General Procedure B, 23 (43.7 mg, $0.20 \mathrm{mmol}, 1.00$ eq.) was converted to $( \pm)-3$. Purification by column chromatography $\left(\mathrm{SiO}_{2}, n\right.$-pentane/ $\left.\mathrm{CH}_{2} \mathrm{Cl}_{2} 7: 3\right)$ yielded the product as

General Procedure B: $\quad$ 1. Reaction: (42.1 mg, $0.19 \mathrm{mmol}, 96 \%)$

2. Reaction: (40.6 mg, $0.19 \mathrm{mmol}, 93 \%)$

$\mathrm{R}_{f}=0.31$ (n-pentane/ $\left.\mathrm{CH}_{2} \mathrm{Cl}_{2} 6: 4\right) ;{ }^{1} \mathrm{H} \mathrm{NMR}\left(400 \mathrm{MHz}, \mathrm{CDCl}_{3}\right): \delta=7.93(\mathrm{~d}, J=9.1 \mathrm{~Hz}, 2 \mathrm{H})$, $6.91(\mathrm{~d}, J=9.0 \mathrm{~Hz}, 2 \mathrm{H}), 5.20(\mathrm{dt}, J=9.5,1.5 \mathrm{~Hz}, 1 \mathrm{H}), 4.22(\mathrm{dq}, J=9.8,6.8 \mathrm{~Hz}, 1 \mathrm{H}), 3.85$ (s, $3 \mathrm{H}), 1.75(\mathrm{~d}, J=1.6 \mathrm{~Hz}, 3 \mathrm{H}), 1.69$ (d, $J=1.6 \mathrm{~Hz}, 3 \mathrm{H}), 1.23$ (d, $J=6.8 \mathrm{~Hz}, 3 \mathrm{H})$ ppm; HR-EIMS: $m / z: 218.13017\left([M]^{+}\right.$, calcd. for $\left.\mathrm{C}_{14} \mathrm{H}_{18} \mathrm{O}_{2}{ }^{+}: 218.13013\right)$; analytical data in agreement with literature. ${ }^{[9]}$

\section{2,4-Dimethyl-1-phenylpent-3-en-1-one (( \pm$)-4)$}

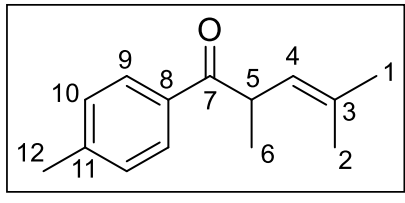

a colorless oil.

\footnotetext{
General Procedure B: $\quad$ 1. Reaction: (33.6 mg, $0.17 \mathrm{mmol}, 83 \%$ )

2. Reaction: (34.4 mg, $0.17 \mathrm{mmol}, 85 \%)$
}

Prepared according to General Procedure B, 24 (40.5 mg, $0.20 \mathrm{mmol}, 1.00$ eq.) was converted to ( \pm )-4. Purification by column chromatography $\left(\mathrm{SiO}_{2}, n\right.$-pentane/ $\left.\mathrm{CH}_{2} \mathrm{Cl}_{2} 7: 3\right)$ yielded the product as 
$\mathrm{R}_{f}=0.41$ (n-pentane/ $\left.\mathrm{CH}_{2} \mathrm{Cl}_{2} 7: 3\right) ;{ }^{1} \mathrm{H} \mathrm{NMR}\left(600 \mathrm{MHz}, \mathrm{CDCl}_{3}\right): \delta=7.85(\mathrm{~d}, J=8.3 \mathrm{~Hz}, 2 \mathrm{H}$, H9), 7.23 (d, $J=7.7 \mathrm{~Hz}, 2 \mathrm{H}, \mathrm{H} 10$ ), 5.21 (ddt, $J=9.5,3.0,1.5 \mathrm{~Hz}, 1 \mathrm{H}, \mathrm{H} 4$ ), 4.25 (dq, $J=9.5$, $6.8 \mathrm{~Hz}, 1 \mathrm{H}, \mathrm{H} 5), 2.39$ (s, 3H, H12), 1.75 (d, J = 1.4 Hz, 3H, H2), 1.69 (d, J = 1.5 Hz, 3H, H1), $1.24(\mathrm{~d}, J=6.8 \mathrm{~Hz}, 3 \mathrm{H}, \mathrm{H} 6) \mathrm{ppm} ;{ }^{13} \mathrm{C} \mathrm{NMR}\left(151 \mathrm{MHz}, \mathrm{CDCl}_{3}\right): \delta=202.1$ (C7), $143.6(\mathrm{C} 11)$, 134.3 (C8), 133.2 (C3), 129.3 (C10), 128.6 (C9), 125.3 (C4), 41.2 (C5), 25.9 (C1), 21.7 (C12), 18.2 (C2), 17.8 (C6) ppm; IR (ATR): $\tilde{v}=$ 2972(w), 2930(w), 2870(w), 1681(s), 1607(m), 1572(w), 1449(w), 1408(w), 1376(w), 1329(w), 1303(w), 1265(w), 1225(m), 1180(m), 1117(w), 1062(w), 967(m), 907(w), 851(w), 830(w), 809(m), 745(m), 685(w) $\mathrm{cm}^{-1}$; HRS-ESIMS: $m / z: 225.12458\left([M+\mathrm{Na}]^{+}\right.$, calcd. for $\left.\mathrm{C}_{14} \mathrm{H}_{18} \mathrm{ONa}^{+}: 225.12498\right)$.

\section{1-(4-Fluorophenyl)-2,4-dimethylpent-3-en-1-one (( \pm )-5)}

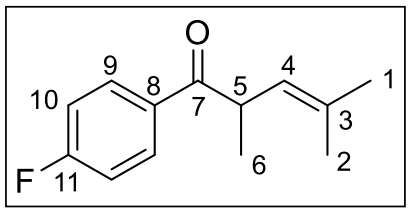

a colorless oil.

Prepared according to General Procedure B, 25 (41.3 mg, $0.20 \mathrm{mmol}, 1.00$ eq.) was converted to ( \pm )-5. Purification by column chromatography $\left(\mathrm{SiO}_{2}, n\right.$-pentane/ $\left.\mathrm{CH}_{2} \mathrm{Cl}_{2} 7: 3\right)$ yielded the product as

General Procedure B: $\quad$ 1. Reaction: (32.1 mg, $0.16 \mathrm{mmol}, 78 \%)$

2. Reaction: (33.7 mg, $0.16 \mathrm{mmol}, 82 \%$ )

$\mathrm{R}_{f}=0.50$ (n-pentane/ $\left.\mathrm{CH}_{2} \mathrm{Cl}_{2} 7: 3\right) ;{ }^{1} \mathrm{H} \mathrm{NMR}\left(500 \mathrm{MHz}, \mathrm{CDCl}_{3}\right): \delta=8.00-7.91(\mathrm{~m}, 2 \mathrm{H}, \mathrm{H} 9)$, $7.13-7.07$ (m, 2H, H10), 5.18 (dp, J=9.5, 1.4 Hz, 1H, H4), 4.20 (dq, J = 9.5, $6.8 \mathrm{~Hz}, 1 \mathrm{H}, \mathrm{H} 5$ ), 1.75 (d, $J=1.4 \mathrm{~Hz}, 3 \mathrm{H}, \mathrm{H} 2), 1.70$ (d, $J=1.5 \mathrm{~Hz}, 3 \mathrm{H}, \mathrm{H} 1), 1.23$ (d, $J=6.8 \mathrm{~Hz}, 3 \mathrm{H}, \mathrm{H} 6)$ ppm; ${ }^{13} \mathrm{C} \mathrm{NMR}\left(126 \mathrm{MHz}, \mathrm{CDCl}_{3}\right): \delta=200.9(\mathrm{C} 7), 165.7$ (d, $\left.J=254.3 \mathrm{~Hz}, \mathrm{C} 11\right), 133.7$ (C3), 133.2 (d, $J=3.0 \mathrm{~Hz}, \mathrm{C} 8), 131.1$ (d, $J=9.1 \mathrm{~Hz}, \mathrm{C} 9), 124.9$ (C4), 115.7 (d, $J=21.9 \mathrm{~Hz}, \mathrm{C} 10), 41.5$ (C5), 25.9 (C1), 18.2 (C2), 17.7 (C6) ppm; ${ }^{19} \mathrm{~F} \mathrm{NMR} \mathrm{(470} \mathrm{MHz,} \mathrm{CDCl}_{3}$ ): $\delta=-105.9$ (tt, $J=8.4$, $5.5 \mathrm{~Hz}) \mathrm{ppm}$; IR (ATR): $\tilde{v}=2972(\mathrm{w}), 2931(\mathrm{w}), 1685(\mathrm{~s}), 1597(\mathrm{~s}), 1506(\mathrm{~m}), 1451(\mathrm{w}), 1409(\mathrm{w})$, 1377(w), 1330(w), 1263(w), 1221(s), 1156(m), 1102(w), 1061(w), 1013(w), 972(m), 907(w), 855(m), 818(w), 758(w), 684(w) $\mathrm{cm}^{-1}$; HR-ESI-MS: $\mathrm{m} / \mathrm{z}: 229.09973\left([\mathrm{M}+\mathrm{Na}]^{+}\right.$, calcd. for $\mathrm{C}_{13} \mathrm{H}_{15} \mathrm{FONa}^{+}$: 229.09991). 


\section{1-(4-Bromophenyl)-2,4-dimethylpent-3-en-1-one (( \pm$)-6)$}

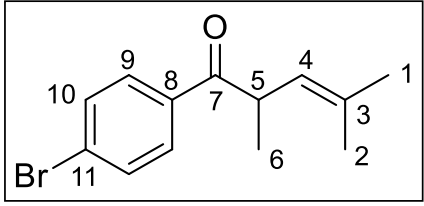

as a white solid.

Prepared according to General Procedure B, 26 (53.4 mg, $0.20 \mathrm{mmol}, 1.00$ eq.) was converted to ( \pm )-6. Purification by column chromatography $\left(\mathrm{SiO}_{2}, n\right.$-pentane/ $\left.\mathrm{CH}_{2} \mathrm{Cl}_{2} 7: 3\right)$ yielded the product

General Procedure B: $\quad$ 1. Reaction: $(26.5 \mathrm{mg}, 0.10 \mathrm{mmol}, 50 \%)$

2. Reaction: (24.7 mg, $0.09 \mathrm{mmol}, 46 \%)$

$\mathrm{R}_{f}=0.56$ (n-pentane/ $\left.\mathrm{CH}_{2} \mathrm{Cl}_{2} 7: 3\right) ;{ }^{1} \mathrm{H} \mathrm{NMR}\left(500 \mathrm{MHz}, \mathrm{CDCl}_{3}\right): \delta=7.81-7.77(\mathrm{~m}, 2 \mathrm{H}, \mathrm{H} 9)$, $7.59-7.56$ (m, 2H, H10), 5.16 (dp, $J=9.5,1.4 \mathrm{~Hz}, 1 \mathrm{H}, \mathrm{H} 4), 4.18$ (dq, $J=9.5,6.7 \mathrm{~Hz}, 1 \mathrm{H}, \mathrm{H} 5$ ), 1.75 (d, $J=1.4 \mathrm{~Hz}, 3 \mathrm{H}, \mathrm{H} 2), 1.70$ (d, $J=1.5 \mathrm{~Hz}, 3 \mathrm{H}, \mathrm{H} 1), 1.23$ (d, $J=6.8 \mathrm{~Hz}, 3 \mathrm{H}, \mathrm{H} 6) \mathrm{ppm}$; ${ }^{13} \mathrm{C} \mathrm{NMR}\left(126 \mathrm{MHz}, \mathrm{CDCl}_{3}\right): \delta=201.4$ (C7), 135.6 (C8), 133.9 (C3), 131.9 (C10), 130.1 (C9), 128.0 (C11), 124.7 (C4), 41.6 (C5), 25.9 (C1), 18.2 (C2), 17.6 (C6) ppm; IR (ATR): $\tilde{v}=$ 2976(w), 2930(m), 2866(w), 1679(s), 1583(s), 1568(m), 1483(w), 1442(m), 1396(m), 1377(w), 1365(w), 1336(m), 1264(m), 1215(m), 1189(w), 1176(m), 1110(w), 1070(m), 1009(m), 967(s), 908(w), 853(m), 833(m), 811(s), 767(m), 733(s), 689(w), 675(m) cm ${ }^{-1}$; HR-ESI-MS: m/z: $289.01986\left([M+\mathrm{Na}]^{+}\right.$, calcd. for $\left.\mathrm{C}_{13} \mathrm{H}_{15} \mathrm{BrONa}^{+}: 289.01985\right) ; \mathrm{m} / z: 291.01776\left([M+\mathrm{Na}]^{+}\right.$, calcd. for $\left.\mathrm{C}_{13} \mathrm{H}_{15} \mathrm{BrONa}^{+}: 291.01780\right) ; \mathrm{Mp}=40.1-40.6^{\circ} \mathrm{C}$.

\section{1-(3-Chlorophenyl)-2,4-dimethylpent-3-en-1-one (( \pm$)-7)$}

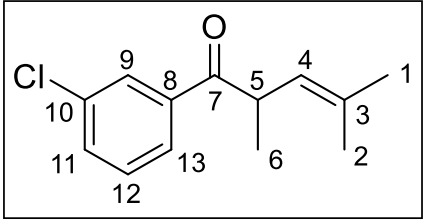

General Procedure B: $\quad$ 1. Reaction: (14.8 mg, $0.07 \mathrm{mmol}, 33 \%)$

2. Reaction: (12.3 mg, $0.06 \mathrm{mmol}, 28 \%)$

$\mathrm{R}_{f}=0.63$ (n-pentane/ $\left.\mathrm{CH}_{2} \mathrm{Cl}_{2} 7: 3\right) ;{ }^{1} \mathrm{H} \mathrm{NMR}\left(600 \mathrm{MHz}, \mathrm{CDCl}_{3}\right): \delta=7.89$ (s, 1H, H9), $7.81-$ 7.78 (m, 1H, H13), $7.51-7.48$ (m, 1H, H11), 7.38 (t, $J=7.9 \mathrm{~Hz}, 1 \mathrm{H}, \mathrm{H} 12), 5.16$ (d, $J=9.6 \mathrm{~Hz}$, 1H, H4), 4.19 (dq, $J=9.6,6.8 \mathrm{~Hz}, 1 \mathrm{H}, \mathrm{H} 5), 1.76$ (d, $J=1.4 \mathrm{~Hz}, 3 \mathrm{H}, \mathrm{H} 2), 1.70$ (d, $J=1.4 \mathrm{~Hz}$, $3 \mathrm{H}, \mathrm{H1}), 1.24$ (d, $J=6.7 \mathrm{~Hz}, 3 \mathrm{H}, \mathrm{H6}) \mathrm{ppm} ;{ }^{13} \mathrm{C} \mathrm{NMR}\left(151 \mathrm{MHz}, \mathrm{CDCl}_{3}\right): \delta=201.2(\mathrm{C} 7), 138.5$ (C8), 134.9 (C10), 134.1 (C3), 132.8 (C11), 129.9 (C12), 128.7 (C9), 126.6 (C13), 124.6 (C4), 41.8 (C5), 25.9 (C1), 18.3 (C2), 17.6 (C6) ppm; IR (ATR): $\tilde{v}=$ 2972(w), 2931(w), 2871(w), 
1687(s), 1571(m), 1448(m), 1422(m), 1376(m), 1325(w), 1259(w), 1215(s), 1187(m), 1076(m), 977(m), 904(w), 842(w), 789(m), 730(m), 702(m), 674(m) $\mathrm{cm}^{-1}$; HR-ESI-MS: m/z: 245.07022 $\left([M+\mathrm{Na}]^{+}\right.$, calcd. for $\mathrm{C}_{13} \mathrm{H}_{15} \mathrm{ClONa}^{+}:$245.07036).

\section{(Z/E)-2-Methyl-1,4-diphenylbut-3-en-1-one (( \pm )-8)}

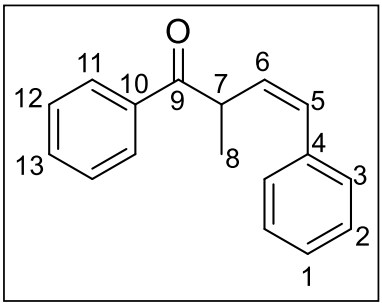

Prepared according to General Procedure B, 28 (47.3 mg, $0.20 \mathrm{mmol}, 1.00$ eq.) was converted to $( \pm)-8$. Purification by column chromatography $\left(\mathrm{SiO}_{2}, n\right.$-pentane/ $\left.\mathrm{CH}_{2} \mathrm{Cl}_{2} 7: 3\right)$ yielded the product as an inseparable mixture of isomers as colorless oil.

General Procedure B: $\quad$ 1. Reaction: (37.0 mg, $0.16 \mathrm{mmol}, 78 \%, Z / E=78: 22$ )

2. Reaction: (37.0 mg, $0.16 \mathrm{mmol}, 78 \%, Z / E=76: 24)$

$\mathrm{R}_{f}=0.33$ (n-pentane/ $\mathrm{CH}_{2} \mathrm{Cl}_{2}$ 7:3); $Z$-isomer (major): ${ }^{1} \mathrm{H}$ NMR $\left(500 \mathrm{MHz}, \mathrm{CDCl}_{3}\right): \delta=7.76-$ 7.73 (m, 2H, H11), $7.52-7.48$ (m, 1H, H13), $7.42-7.38$ (m, 2H, H2), $7.36-7.32$ (m, 3H, H12/H1), $7.29-7.27$ (m, 2H, H3), 6.59 (d, $J=11.4 \mathrm{~Hz}, 1 \mathrm{H}, \mathrm{H} 5), 5.77$ (dd, $J=11.4,10.4 \mathrm{~Hz}$, 1H, H6), 4.66 (dqd, $J=10.4,6.8,0.8 \mathrm{~Hz}, 1 \mathrm{H}, \mathrm{H} 7), 1.43$ (d, $J=6.8 \mathrm{~Hz}, 3 \mathrm{H}, \mathrm{H} 8$ ) ppm; ${ }^{13} \mathrm{C} \mathrm{NMR}$ (126 MHz, $\mathrm{CDCl}_{3}$ ): $\delta=201.6$ (C9), 137.2 (C4), 136.1 (C10), 133.1 (C13), 132.3 (C6), 130.5 (C5), 128.7 (C2), 128.6 (C11/C12), 128.6 (C11/C12), 128.5 (C3), 127.4 (C1), 40.8 (C7), 18.1 (C8) ppm; E-isomer (minor): ${ }^{1} \mathrm{H}$ NMR (500 MHz, $\left.\mathrm{CDCl}_{3}\right): \delta=8.05-8.02(\mathrm{~m}, 2 \mathrm{H}), 7.58-7.54$ (m, 1H), $7.50-7.44(\mathrm{~m}, 2 \mathrm{H}), 7.36-7.34(\mathrm{~m}, 2 \mathrm{H}), 7.32-7.30(\mathrm{~m}, 2 \mathrm{H}), 7.23-7.19(\mathrm{~m}, 1 \mathrm{H})$, $6.54(\mathrm{~d}, J=16.0 \mathrm{~Hz}, 1 \mathrm{H}), 6.38(\mathrm{dd}, J=16.0,8.2 \mathrm{~Hz}, 1 \mathrm{H}), 4.34(\mathrm{dqd}, J=7.8,6.8,1.0 \mathrm{~Hz}, 1 \mathrm{H})$, $1.44(\mathrm{~d}, J=6.9 \mathrm{~Hz}, 3 \mathrm{H})$ ppm; HR-EI-MS: $m / z: 236.11965\left([M]^{+}\right.$, calcd. for $\mathrm{C}_{17} \mathrm{H}_{16} \mathrm{O}^{+}$: 236.11957); analytical data of the $(E)$-isomer in agreement with literature. ${ }^{[10]}$

\section{3-Cyclohexylidene-2-methyl-1-phenylpropan-1-one (( \pm$)-9)$}

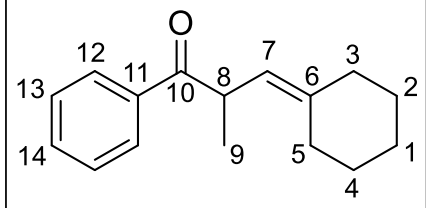

General Procedure B: $\quad$ 1. Reaction: (34.2 mg, $0.15 \mathrm{mmol}, 75 \%)$

2. Reaction: (33.6 mg, $0.15 \mathrm{mmol}, 74 \%$ ) 
$\mathrm{R}_{f}=0.50$ (n-pentane/ $\mathrm{CH}_{2} \mathrm{Cl}_{2}$ 7:3); ${ }^{1} \mathrm{H}$ NMR $\left(500 \mathrm{MHz}, \mathrm{CDCl}_{3}\right): \delta=7.96-7.93(\mathrm{~m}, 2 \mathrm{H}, \mathrm{H} 12)$, 7.55 - 7.50 (m, 1H, H14), 7.46 - 7.41 (m, 2H, H13), 5.13 (d, J=9.5 Hz, 1H, H7), 4.32 (dq, $J$ = 9.5, 6.7 Hz, 1H, H8), $2.32-2.22$ (m, 2H, H5), $2.11-2.00$ (m, 2H, H3), $1.56-1.47$ (m, 6H, $\mathrm{H} 1 / \mathrm{H} 2 / \mathrm{H} 4), 1.25$ (d, $J=6.7 \mathrm{~Hz}, 3 \mathrm{H}, \mathrm{H} 9) \mathrm{ppm} ;{ }^{13} \mathrm{C} \mathrm{NMR}\left(126 \mathrm{MHz}, \mathrm{CDCl}_{3}\right): \delta=202.6(\mathrm{C} 10)$, 141.5 (C6), 136.9 (C11), 132.8 (C14), 128.6 (C12), 128.5 (C13), 121.8 (C7), 40.6 (C8), 37.2 (C3), 29.4 (C5), 28.5 (C1/C2/C4), 27.8 (C1/C2/C4), 26.9 (C1/C2/C4), 18.1 (C9) ppm; IR (ATR): $\tilde{v}=2928(\mathrm{~m}), 2851(\mathrm{w}), 1671(\mathrm{~s}), 1595(\mathrm{w}), 1578(\mathrm{w}), 1447(\mathrm{~m}), 1368(\mathrm{w}), 1343(\mathrm{w})$, 1335(w), 1324(w), 1306(w), 1267(w), 1221(m), 1199(m), 1147(m), 1104(w), 1090(w), 1078(w), 1028(w), 1000(w), 990(w), 969(s), 938(m), 920(w), 897(w), 859(w), 848(m), 793(m), 749(w), 699(s) cm ${ }^{-1}$; HR-EI-MS: $m / z: 228.15091$ ([M] $]^{+}$, calcd. for $\mathrm{C}_{16} \mathrm{H}_{20} \mathrm{O}^{+}:$228.15087).

\section{3-Cyclopentylidene-2-methyl-1-phenylpropan-1-one (( \pm$)-10)$}

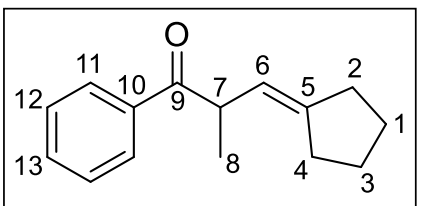

product as a colorless oil.

General Procedure B: $\quad$ 1. Reaction: (33.2 mg, $0.15 \mathrm{mmol}, 77 \%$ )

2. Reaction: (30.9 mg, $0.14 \mathrm{mmol}, 72 \%)$

$\mathrm{R}_{f}=0.49\left(n\right.$-pentane $\left./ \mathrm{CH}_{2} \mathrm{Cl}_{2} 7: 3\right) ;{ }^{1} \mathrm{H} \mathrm{NMR}\left(500 \mathrm{MHz}, \mathrm{CDCl}_{3}\right): \delta=7.95(\mathrm{dd}, J=8.4,1.4 \mathrm{~Hz}$, 2H, H11), $7.57-7.50$ (m, 1H, H13), $7.48-7.41$ (m, 2H, H12), 5.35 (dp, J = 9.3, $2.3 \mathrm{~Hz}, 1 \mathrm{H}$, H6), 4.17 (dq, $J=9.4,6.8$ Hz, 1H, H7), $2.38-2.24$ (m, 2H, H4), 2.27 - 2.20 (m, 2H, H2), 1.70 $(\mathrm{p}, J=6.9 \mathrm{~Hz}, 2 \mathrm{H}, \mathrm{H} 3), 1.67-1.53(\mathrm{~m}, 2 \mathrm{H}, \mathrm{H} 1), 1.27$ (d, $J=6.8 \mathrm{~Hz}, 3 \mathrm{H}, \mathrm{H} 8) \mathrm{ppm} ;{ }^{13} \mathrm{C} \mathrm{NMR}$ (126 MHz, $\left.\mathrm{CDCl}_{3}\right): \delta=202.7$ (C9), 145.5 (C5), 137.0 (C10), 132.9 (C13), 128.6 (C12), 128.5 (C11), 120.6 (C6), 42.9 (C7), 33.9 (C2), 29.1 (C4), 26.5 (C3), 26.3 (C1), 17.6 (C6) ppm; IR (ATR): $\tilde{v}=2953(\mathrm{~m}), 2868(\mathrm{w}), 1683(\mathrm{~s}), 1596(\mathrm{~m}), 1579(\mathrm{w}), 1448(\mathrm{~m}), 1369(\mathrm{w}), 1330(\mathrm{w})$, 1258(w), 1215(s), 1179(w), 1160(w), 1074(w), 1001(w), 971(s), 901(w), 858(w), 762(w), 703(s), 660(w) cm ${ }^{-1}$; HR-EI-MS: $m / z: 214.13531\left([M]^{+}\right.$, calcd. for $\mathrm{C}_{15} \mathrm{H}_{18} \mathrm{O}^{+}:$214.13522). 


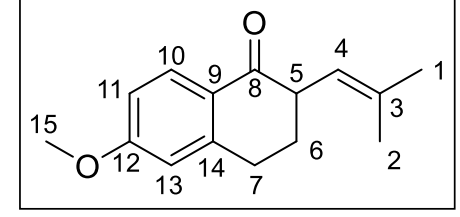

General Procedure B: $\quad$ 1. Reaction: (40.0 mg, $0.17 \mathrm{mmol}, 87 \%$ )

2. Reaction: (42.0 mg, $0.18 \mathrm{mmol}, 91 \%$ )

$\mathrm{R}_{f}=0.42\left(\mathrm{CH}_{2} \mathrm{Cl}_{2}\right) ;{ }^{1} \mathrm{H} \mathrm{NMR}\left(600 \mathrm{MHz}, \mathrm{CDCl}_{3}\right): \delta=8.00(\mathrm{~d}, J=8.7 \mathrm{~Hz}, 1 \mathrm{H}, \mathrm{H} 10), 6.81(\mathrm{dd}$, $J=8.8,2.5 \mathrm{~Hz}, 1 \mathrm{H}, \mathrm{H} 11), 6.68(\mathrm{~d}, J=2.6 \mathrm{~Hz}, 1 \mathrm{H}, \mathrm{H} 13), 5.30(\mathrm{dp}, J=8.6,1.4 \mathrm{~Hz}, 1 \mathrm{H}, \mathrm{H} 4)$, 3.84 (s, 3H, H15), 3.34 (ddd, $J=10.6,8.6,4.6$ Hz, 1H, H5), 3.00 - 2.97 (m, 2H, H7), 2.17 2.11 (m, 1H, H6), 1.97 (dddd, $J=13.3,10.7,8.5,6.3 \mathrm{~Hz}, 1 \mathrm{H}, \mathrm{H6}$ '), 1.77 (d, $J=1.5 \mathrm{~Hz}, 3 \mathrm{H}$, H1), $1.71(\mathrm{~d}, J=1.4 \mathrm{~Hz}, 3 \mathrm{H}, \mathrm{H} 2) \mathrm{ppm} ;{ }^{13} \mathrm{C} \mathrm{NMR}\left(151 \mathrm{MHz}, \mathrm{CDCl}_{3}\right): \delta=198.0(\mathrm{C} 8), 163.5$ (C12), 146.5 (C14), 135.4 (C3), 130.2 (C10), 126.3 (C9), 121.9 (C4), 113.2 (C11), 112.5 (C13), 55.5 (C15), 47.4 (C5), 30.3 (C6), 28.9 (C7), 26.0 (C1), 18.4 (C2) ppm; IR (ATR): $\tilde{v}=2930(w)$, 2858(w), 1671(s), 1597(s), 1572(m), 1494(m), 1445(m), 1375(w), 1356(m), 1343(m), 1302(w), 1245(s), 1230(s), 1186(w), 1153(m), 1128(m), 1097(m), 1026(m), 1000(w), 928(m), 883(m), 850(m), 816(m), 759(m), 724(w), 667(w) cm ${ }^{-1}$; HR-ESI-MS: m/z: $253.11933\left([M+N a]^{+}\right.$, calcd. for $\left.\mathrm{C}_{15} \mathrm{H}_{18} \mathrm{O}_{2} \mathrm{Na}^{+}: 253.11990\right) ; \mathrm{Mp}=77.5-78.9{ }^{\circ} \mathrm{C}$.

\section{2-(2-Methylprop-1-en-1-yl)-3,4-dihydronaphthalen-1(2H)-one (( \pm -12)}

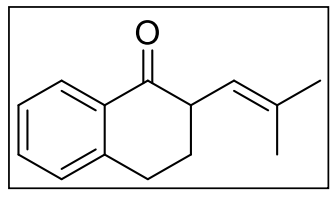

colorless oil.

General Procedure B: $\quad$ 1. Reaction: (27.8 mg, $0.14 \mathrm{mmol}, 69 \%)$

2. Reaction: (24.7 mg, $0.12 \mathrm{mmol}, 62 \%)$ 1H), $7.46(\mathrm{td}, J=7.5,1.5 \mathrm{~Hz}, 1 \mathrm{H}), 7.32-7.27(\mathrm{~m}, 1 \mathrm{H}), 7.24(\mathrm{~d}, J=7.8 \mathrm{~Hz}, 1 \mathrm{H}), 5.32(\mathrm{dt}, J=$ 8.6, $1.5 \mathrm{~Hz}, 1 \mathrm{H}), 3.40$ (ddd, $J=11.0,8.6,4.6 \mathrm{~Hz}, 1 \mathrm{H}), 3.04(\mathrm{~m}, 2 \mathrm{H}), 2.17$ (dq, $J=13.3,4.8 \mathrm{~Hz}$, 1H), 2.01 (dddd, $J=13.4,11.0,8.6,6.3 \mathrm{~Hz}, 1 \mathrm{H}), 1.79$ (d, $J=1.5 \mathrm{~Hz}, 3 \mathrm{H}), 1.71(\mathrm{~d}, J=1.4 \mathrm{~Hz}$, 
3H) ppm; HR-EI-MS: $m / z: 200.11938\left([M]^{+}\right.$, calcd. for $\left.\mathrm{C}_{14} \mathrm{H}_{16} \mathrm{O}^{+}: 200.11957\right)$; analytical data in agreement with literature. ${ }^{[9]}$

\section{7-Fluoro-2-(2-methylprop-1-en-1-yl)-3,4-dihydronaphthalen-1(2H)-one $(( \pm)-13)$}

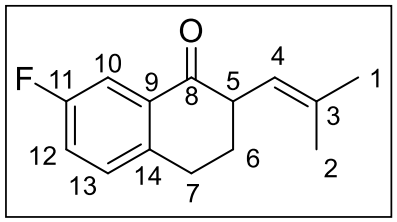

a colorless oil.

Prepared according to General Procedure B, 34 (43.7 mg, $0.20 \mathrm{mmol}, 1.00$ eq.) was converted to $( \pm)-13$. Purification by column chromatography $\left(\mathrm{SiO}_{2}, n\right.$-pentane/ $\left.\mathrm{CH}_{2} \mathrm{Cl}_{2} 7: 3\right)$ yielded the product as

General Procedure B: 1. Reaction: (17.8 mg, $0.08 \mathrm{mmol}, 41 \%)$

2. Reaction: (22.3 mg, $0.10 \mathrm{mmol}, 51 \%)$

$\mathrm{R}_{f}=0.35\left(n\right.$-pentane $\left./ \mathrm{CH}_{2} \mathrm{Cl}_{2} 6: 4\right) ;{ }^{1} \mathrm{H} \mathrm{NMR}\left(500 \mathrm{MHz}, \mathrm{CDCl}_{3}\right): \delta=7.68(\mathrm{dd}, J=9.2,2.8 \mathrm{~Hz}$, 1H, H10), $7.24-7.20$ (m, 1H, H13), 7.16 (td, $J=8.2,2.8$ Hz, 1H, H12), 5.29 (ddq, $J=8.6,2.9$, $1.4 \mathrm{~Hz}, 1 \mathrm{H}, \mathrm{H} 4), 3.38$ (ddd, $J=10.9,8.5,4.6 \mathrm{~Hz}, 1 \mathrm{H}, \mathrm{H} 5), 3.02-2.98$ (m, 2H, H7), 2.20 - 2.13 (m, 1H, H6), 1.99 (dddd, $J=13.4,11.0,8.0,6.8 \mathrm{~Hz}, 1 \mathrm{H}, \mathrm{H6}$ '), 1.78 (d, $J=1.5 \mathrm{~Hz}, 3 \mathrm{H}, \mathrm{H1}$ ), 1.71 (d, $J=1.4 \mathrm{~Hz}, 3 \mathrm{H}, \mathrm{H} 2) \mathrm{ppm} ;{ }^{13} \mathrm{C} \mathrm{NMR}\left(126 \mathrm{MHz}, \mathrm{CDCl}_{3}\right): \delta=198.1$ (d, $\left.J=1.7 \mathrm{~Hz}, \mathrm{C} 8\right)$, 161.7 (d, $J=246.0 \mathrm{~Hz}, \mathrm{C} 11), 139.8$ (d, $J=3.1 \mathrm{~Hz}, \mathrm{C} 14), 136.0$ (C3), 134.3 (d, $J=6.0 \mathrm{~Hz}, \mathrm{C} 9$ ), 130.6 (d, $J=7.0 \mathrm{~Hz}, \mathrm{C} 13), 121.1(\mathrm{C} 4), 120.6$ (d, $J=22.2 \mathrm{~Hz}, \mathrm{C} 12), 113.6$ (d, $J=21.8 \mathrm{~Hz}, \mathrm{C} 10)$, 47.4 (d, $J=1.2 \mathrm{~Hz}, \mathrm{C} 5), 30.3$ (C6), 27.9 (C7), 26.0 (C1), 18.5 (C2) ppm; ${ }^{19} \mathrm{~F}$ NMR (470 MHz, $\left.\mathrm{CDCl}_{3}\right): \delta=-115.4(\mathrm{dddt}, J=9.4,8.0,5.2,1.4 \mathrm{~Hz}) \mathrm{ppm}$; IR (ATR): $\tilde{v}=2929(\mathrm{w}), 1686(\mathrm{~s})$, 1612(w), 1588(w), 1490(s), 1422(m), 1376(w), 1357(w), 1311(m), 1255(s), 1238(s), 1157(m), 1039(w), 1003(w), 892(s), 871(m), 816(s), 758(w), 730(s), 714(w), 702(w), 677(w) cm ${ }^{-1}$; HRESI-MS: $m / z: 241.09972\left([M+\mathrm{Na}]^{+}\right.$, calcd. for $\mathrm{C}_{14} \mathrm{H}_{15} \mathrm{OFNa}^{+}:$241.09991).

\section{7-Bromo-2-(2-methylprop-1-en-1-yl)-3,4-dihydronaphthalen-1(2H)-one (( $( \pm)-14)$}

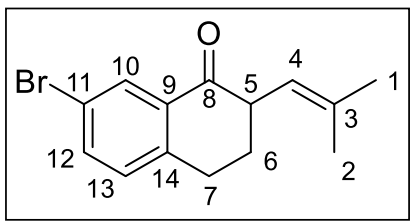

General Procedure B: $\quad$ 1. Reaction: (20.5 mg, $0.07 \mathrm{mmol}, 37 \%$ )

2. Reaction: (19.4 mg, $0.07 \mathrm{mmol}, 35 \%)$

$\mathrm{R}_{f}=0.40\left(n\right.$-pentane/ $\left.\mathrm{CH}_{2} \mathrm{Cl}_{2} 6: 4\right) ;{ }^{1} \mathrm{H} \mathrm{NMR}\left(500 \mathrm{MHz}, \mathrm{CDCl}_{3}\right): \delta=8.14(\mathrm{~d}, J=2.3 \mathrm{~Hz}, 1 \mathrm{H}$, H10), 7.55 (dd, $J=8.2,2.2 \mathrm{~Hz}, 1 \mathrm{H}, \mathrm{H} 12), 7.13$ (d, $J=8.2 \mathrm{~Hz}, 1 \mathrm{H}, \mathrm{H} 13), 5.28$ (ddq, $J=8.5$, 
2.8, 1.4 Hz, 1H, H4), 3.38 (ddd, $J=10.9,8.5,4.6 \mathrm{~Hz}, 1 \mathrm{H}, \mathrm{H} 5), 3.00$ - 2.96 (m, 2H, H7), 2.21 - 2.12 (m, 1H, H6), 1.99 (dddd, $J=13.4,10.9,8.2,6.5$ Hz, 1H, H6'), 1.78 (d, $J=1.4$ Hz, 3H, $\mathrm{H} 1), 1.70$ (d, $J=1.4 \mathrm{~Hz}, 3 \mathrm{H}, \mathrm{H} 2) \mathrm{ppm} ;{ }^{13} \mathrm{C} \mathrm{NMR}\left(126 \mathrm{MHz}, \mathrm{CDCl}_{3}\right): \delta=197.8$ (C8), 142.7 (C14), 136.1 (C3), 136.0 (C12), 134.2 (C9), 130.7 (C13), 130.5 (C10), 121.0 (C4), 120.7 (C11), 47.4 (C5), 30.0 (C6), 28.1 (C7), 26.0 (C1), 18.5 (C2) ppm; IR (ATR): $\tilde{v}=2928(w), 2861(w)$, 1683(s), 1589(m), 1473(m), 1434(m), 1402(m), 1375(w), 1357(w), 1302(m), 1259(m), 1209(s), 1175(m), 1133(w), 1112(w), 1075(w), 996(w), 938(w), 904(m), 880(w), 846(w), 807(s), 756(m), 735(w), 716(w), 679(w) $\mathrm{cm}^{-1}$; HR-ESI-MS: m/z: $301.01980\left([M+\mathrm{Na}]^{+}\right.$, calcd. for $\left.\mathrm{C}_{14} \mathrm{H}_{15} \mathrm{OBrNa}^{+}: 301.01985\right) ; \mathrm{m} / z: 303.01759\left([M+\mathrm{Na}]^{+}\right.$, calcd. for $\left.\mathrm{C}_{14} \mathrm{H}_{15} \mathrm{OBrNa}^{+}: 303.01780\right)$, $\mathrm{Mp}=47.6-48.6^{\circ} \mathrm{C}$.

\section{5,7-Dimethyl-2-(2-methylprop-1-en-1-yl)-3,4-dihydronaphthalen-1(2H)-one (( \pm$)-15)$}

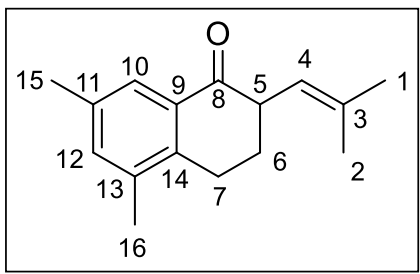

General Procedure B: $\quad$ 1. Reaction: (37.7 mg, $0.16 \mathrm{mmol}, 82 \%)$

2. Reaction: (37.8 mg, $0.17 \mathrm{mmol}, 83 \%$ )

$\mathrm{R}_{f}=0.32\left(n\right.$-pentane $\left./ \mathrm{CH}_{2} \mathrm{Cl}_{2} 6: 4\right) ;{ }^{1} \mathrm{H}$ NMR $\left(600 \mathrm{MHz}, \mathrm{CDCl}_{3}\right): \delta=7.74-7.71(\mathrm{~m}, 1 \mathrm{H}, \mathrm{H} 10)$, $7.19-7.16$ (m, 1H, H12), 5.33 (dp, $J=8.6,1.4 \mathrm{~Hz}, 1 \mathrm{H}, \mathrm{H} 4), 3.38$ (ddd, $J=10.8,8.5,4.5 \mathrm{~Hz}$, 1H, H5), 2.94 (dt, $J=17.2,5.1 \mathrm{~Hz}, 1 \mathrm{H}, \mathrm{H} 7$ ), 2.81 (ddd, $J=17.1,9.7,5.0 \mathrm{~Hz}, 1 \mathrm{H}, \mathrm{H} 7^{\prime}$ ), 2.32 (s, 3H, H15), 2.27 (s, 3H, H16), $2.21-2.15$ (m, 1H, H6), 1.99 (dddd, $J=13.4,10.8,9.7,4.8$ Hz, 1H, H6'), 1.78 (d, $J=1.5 \mathrm{~Hz}, 3 \mathrm{H}, \mathrm{H} 1), 1.71$ (d, $J=1.4 \mathrm{~Hz}, 3 \mathrm{H}, \mathrm{H} 2) \mathrm{ppm} ;{ }^{13} \mathrm{C}$ NMR $(151 \mathrm{MHz}$, $\left.\mathrm{CDCl}_{3}\right): \delta=200.0(\mathrm{C} 8), 139.4(\mathrm{C} 14), 136.3(\mathrm{C} 13), 135.8$ (C11), 135.7 (C12), 135.4 (C3), 132.7 (C9), 125.6 (C10), 121.5 (C4), 47.0 (C5), 29.6 (C6), 26.0 (C1), 25.2 (C7), 21.0 (C15), 19.4 (C16), 18.4 (C2) ppm; IR (ATR): $\tilde{v}=2919(\mathrm{~m}), 2860(\mathrm{w}), 1680(\mathrm{~s}), 1610(\mathrm{~m}), 1475(\mathrm{~m}), 1447(\mathrm{~m})$, 1410(w), 1379(m), 1359(w), 1314(m), 1288(m), 1241(w), 1195(w), 1156(m), 1101(w), 1040(w), 1012(w), 973(w), 940(w), 912(m), 887(m), 874(m), 855(w), 819(m), 756(m), 730(s), 696(w) $\mathrm{cm}^{-1}$; HR-ESI-MS: $m / z: 251.14040\left([M+\mathrm{Na}]^{+}\right.$, calcd. for $\mathrm{C}_{16} \mathrm{H}_{20} \mathrm{ONa}^{+}$: 251.14063). 


\section{3-(2-Methylprop-1-en-1-yl)chroman-4-one (( \pm$)-16)$}

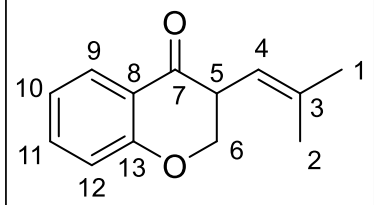

Prepared according to General Procedure B, 37 (40.5 mg, $0.20 \mathrm{mmol}, 1.00$ eq.) was converted to ( \pm )-16. Purification by column chromatography $\left(\mathrm{SiO}_{2}, n\right.$-pentane/ $\left.\mathrm{CH}_{2} \mathrm{Cl}_{2} 7: 3\right)$ yielded the product as a

colorless oil.

General Procedure B: $\quad$ 1. Reaction: (37.2 mg, $0.18 \mathrm{mmol}, 92 \%)$

2. Reaction: (37.8 mg, $0.19 \mathrm{mmol}, 93 \%)$

$\mathrm{R}_{f}=0.30$ (n-pentane/ $\left.\mathrm{CH}_{2} \mathrm{Cl}_{2} 6: 4\right) ;{ }^{1} \mathrm{H} \mathrm{NMR}\left(600 \mathrm{MHz}, \mathrm{CDCl}_{3}\right): \delta=7.90(\mathrm{dd}, J=7.8,1.8 \mathrm{~Hz}$, 1H, H9), 7.46 (ddd, $J=8.8,7.2,1.8 \mathrm{~Hz}, 1 \mathrm{H}, \mathrm{H} 11$ ), 7.00 (ddd, $J=8.0,7.1,1.0 \mathrm{~Hz}, 1 \mathrm{H}, \mathrm{H} 10$ ), 6.96 (dd, $J=8.4,1.1 \mathrm{~Hz}, 1 \mathrm{H}, \mathrm{H} 12), 5.23$ (dp, $J=8.4,1.4 \mathrm{~Hz}, 1 \mathrm{H}, \mathrm{H} 4), 4.44$ (dd, $J=11.3$, $5.0 \mathrm{~Hz}, 1 \mathrm{H}, \mathrm{H6}$ ), 4.23 (dd, $J=11.3,10.2 \mathrm{~Hz}, 1 \mathrm{H}, \mathrm{H6}$ '), 3.63 (ddd, $J=10.2,8.6,4.9 \mathrm{~Hz}, 1 \mathrm{H}$, H5), 1.79 (d, $J=1.5 \mathrm{~Hz}, 3 \mathrm{H}, \mathrm{H} 1), 1.75$ (d, $J=1.4 \mathrm{~Hz}, 3 \mathrm{H}, \mathrm{H} 2) \mathrm{ppm} ;{ }^{13} \mathrm{C}$ NMR $(151 \mathrm{MHz}$, $\left.\mathrm{CDCl}_{3}\right): \delta=193.2$ (C7), 161.6 (C13), 139.0 (C3), 135.8 (C11), 127.7 (C9), 121.5 (C10), 121.0 (C8), 117.8 (C12), 115.9 (C4), 70.8 (C6), 46.2 (C5), 26.0 (C1), 18.7 (C2) ppm; IR (ATR): $\tilde{v}=$ 2973(w), 2914(w), 2869(w), 1689(s), 1604(s), 1579(m), 1477(s), 1464(s), 1455(s), 1378(m), 1354(w), 1322(m), 1305(s), 1286(s), 1214(m), 1148(m), 1129(m), 1106(w), 1076(w), 1056(w), 1033(m), 1004(m), 941(m), 918(w), 848(m), 821(w), 771(m), 757(s), 7300(w), 687(w), 657(w) $\mathrm{cm}^{-1}$; HR-ESI-MS: $m / z: 225.08843\left([M+\mathrm{Na}]^{+}\right.$, calcd. for $\left.\mathrm{C}_{13} \mathrm{H}_{15} \mathrm{O}_{2} \mathrm{Na}^{+}: 225.08860\right)$.

\section{2-(2-Methylprop-1-en-1-yl)-2,3-dihydro-1H-inden-1-one (( \pm$)-17)$}

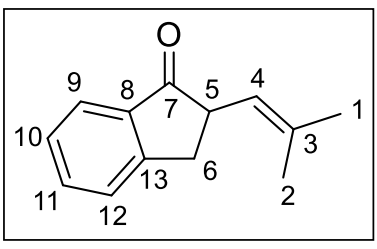

General Procedure B: $\quad$ 1. Reaction: $(29.7 \mathrm{mg}, 0.16 \mathrm{mmol}, 80 \%)$

2. Reaction: (29.9 mg, $0.16 \mathrm{mmol}, 80 \%)$

$\mathrm{R}_{f}=0.20\left(n\right.$-pentane/ $\left.\mathrm{CH}_{2} \mathrm{Cl}_{2} 6: 4\right) ;{ }^{1} \mathrm{H} \mathrm{NMR}\left(500 \mathrm{MHz}, \mathrm{CDCl}_{3}\right): \delta=7.75(\mathrm{~d}, J=7.6 \mathrm{~Hz}, 1 \mathrm{H}$, H9), 7.57 (td, $J=7.5,1.3 \mathrm{~Hz}, 1 \mathrm{H}, \mathrm{H} 11), 7.44$ (dt, $J=7.7,1.0 \mathrm{~Hz}, 1 \mathrm{H}, \mathrm{H} 12), 7.36$ (td, $J=7.5$, $1.0 \mathrm{~Hz}, 1 \mathrm{H}, \mathrm{H} 10), 5.10$ (dt, $J=8.6,1.4 \mathrm{~Hz}, 1 \mathrm{H}, \mathrm{H} 4), 3.55$ (td, $J=8.4,4.2 \mathrm{~Hz}, 1 \mathrm{H}, \mathrm{H} 5), 3.45$ (dd, $J=17.0,8.1 \mathrm{~Hz}, 1 \mathrm{H}, \mathrm{H} 6), 2.85$ (dd, $J=17.0,4.2 \mathrm{~Hz}, 1 \mathrm{H}, \mathrm{H6}$ '), $1.80-1.76$ (m, 6H, H1/H2) ppm; ${ }^{13} \mathrm{C} \mathrm{NMR}\left(126 \mathrm{MHz}, \mathrm{CDCl}_{3}\right.$ ): $\delta=207.7$ (C7), 153.7 (C13), 136.8 (C3), 136.6 (C8), 134.8 
(C11), 127.5 (C10), 126.6 (C12), 124.2 (C9), 121.9 (C4), 47.7 (C5), 34.6 (C6), 25.9 (C1), 18.8 (C2) ppm; IR (ATR): $\tilde{v}=2918(\mathrm{w}), 1705(\mathrm{~s}), 1651(\mathrm{~m}), 1607(\mathrm{~m}), 1465(\mathrm{~m}), 1435(\mathrm{w}), 1376(\mathrm{w})$, 1325(m), 1294(m), 1268(m), 1204(w), 1152(m), 1093(m), 998(w), 901(w), 854(w), 814(w), 781(m), 749(s), 739(s), 721(m), 675(m) cm ${ }^{-1}$; HR-EI-MS: m/z: $186.10386\left([M]^{+}\right.$, calcd. for $\mathrm{C}_{13} \mathrm{H}_{14} \mathrm{O}^{+}:$186.10392).

\section{6-(2-Methylprop-1-en-1-yl)-6,7,8,9-tetrahydro-5H-benzo[7]annulen-5-one $(( \pm)-18)$}

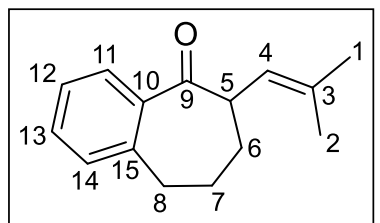

Prepared according to General Procedure B, 39 (42.9 mg, $0.20 \mathrm{mmol}, 1.00$ eq.) was converted to $( \pm)-\mathbf{1 8}$. Purification by column chromatography $\left(\mathrm{SiO}_{2}, n\right.$-pentane/ $\left.\mathrm{CH}_{2} \mathrm{Cl}_{2} 7: 3\right)$ yielded the product as a colorless oil.

General Procedure B: $\quad$ 1. Reaction: (40.9 mg, $0.19 \mathrm{mmol}, 95 \%)$

2. Reaction: (39.1 mg, $0.18 \mathrm{mmol}, 91 \%)$

$\mathrm{R}_{f}=0.32\left(n\right.$-pentane/ $\left.\mathrm{CH}_{2} \mathrm{Cl}_{2} 6: 4\right) ;{ }^{1} \mathrm{H} \mathrm{NMR}\left(600 \mathrm{MHz}, \mathrm{CDCl}_{3}\right): \delta=7.61(\mathrm{dd}, J=7.7,1.5 \mathrm{~Hz}$, 1H, H11), 7.37 (td, $J=7.5,1.5 \mathrm{~Hz}, 1 \mathrm{H}, \mathrm{H} 13), 7.29-7.25$ (m, 1H, H12), 7.21 (d, $J=7.6 \mathrm{~Hz}$, 1H, H14), 5.50 (dp, $J=8.7,1.5 \mathrm{~Hz}, 1 \mathrm{H}, \mathrm{H} 4), 3.72$ (ddd, $J=10.3,8.7,5.5 \mathrm{~Hz}, 1 \mathrm{H}, \mathrm{H} 5), 3.05$ (ddd, $J=16.2,10.4,2.9 \mathrm{~Hz}, 1 \mathrm{H}, \mathrm{H} 8$ ), 2.97 (ddd, $J=15.9,6.8,2.8 \mathrm{~Hz}, 1 \mathrm{H}, \mathrm{H} 8$ '), 2.07 (dtt, $J=$ 14.1, 7.3, 3.7 Hz, 1H, H7), 1.93 - 1.86 (m, 1H, H6), 1.79 - 1.69 (m, 5H, H1/H6'/H7'), 1.61 (d, $J=1.4 \mathrm{~Hz}, 3 \mathrm{H}, \mathrm{H} 2) \mathrm{ppm} ;{ }^{13} \mathrm{C} \mathrm{NMR}\left(151 \mathrm{MHz}, \mathrm{CDCl}_{3}\right): \delta=206.8$ (C9), 141.7 (C15), 140.3 (C10), 133.4 (C3), 131.3 (C13), 129.9 (C14), 128.5 (C11), 126.5 (C12), 122.7 (C4), 49.4 (C5), 34.0 (C8), 31.7 (C6), 25.9 (C1), 25.5 (C7), 18.4 (C2) ppm; IR (ATR): $\tilde{v}=2929(\mathrm{~m}), 2860(\mathrm{w})$, 1683(s), 1598(m), 1446(m), 1374(m), 1347(w), 1307(w), 1275(m), 1220(m), 1201(m), 1183(m), 1160(w), 1124(w), 1101(w), 1074(w), 1057(w), 968(m), 862(w), 822(w), 779(m), 762(m), 734(s), 681(w) $\mathrm{cm}^{-1}$; HR-EI-MS: m/z: $214.13517\left([M]^{+}\right.$, calcd. for $\mathrm{C}_{15} \mathrm{H}_{18} \mathrm{O}^{+}$: 214.13522).

\section{6-(2-Ethylbut-1-en-1-yl)-6,7,8,9-tetrahydro-5H-benzo[7]annulen-5-one (( \pm$)-19)$}

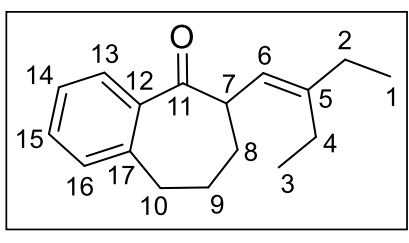

Prepared according to General Procedure B, 40 (48.5 mg, $0.20 \mathrm{mmol}, 1.00$ eq.) was converted to $( \pm)-19$. Purification by column chromatography $\left(\mathrm{SiO}_{2}, n\right.$-pentane/ $\left.\mathrm{CH}_{2} \mathrm{Cl}_{2} 7: 3\right)$ yielded the product as a colorless oil. 
General Procedure B: $\quad$ 1. Reaction: (45.2 mg, $0.19 \mathrm{mmol}, 93 \%)$

2. Reaction: (44.2 mg, $0.18 \mathrm{mmol}, 91 \%)$

$\mathrm{R}_{f}=0.42\left(n\right.$-pentane/ $\left.\mathrm{CH}_{2} \mathrm{Cl}_{2} 6: 4\right) ;{ }^{1} \mathrm{H} \mathrm{NMR}\left(400 \mathrm{MHz}, \mathrm{CDCl}_{3}\right): \delta=7.61(\mathrm{dd}, J=7.7,1.5 \mathrm{~Hz}$, 1H, H13), 7.37 (td, $J=7.5,1.5 \mathrm{~Hz}, 1 \mathrm{H}, \mathrm{H} 15), 7.27$ (t, $J=7.5 \mathrm{~Hz}, 1 \mathrm{H}, \mathrm{H} 14), 7.22$ (d, $J=7.6$ Hz, 1H, H16), 5.46 (d, $J=9.0$ Hz, 1H, H6), 3.77 (td, $J=9.5,5.7$ Hz, 1H, H7), 3.12 - 2.92 (m, 2H, H10), $2.12-2.02$ (m, 3H, H2/H9), 2.00 (q, $J=7.6$ Hz, 2H, H4), $1.95-1.82$ (m, 1H, H8), $1.84-1.68$ (m, 2H, H8'/H9'), 1.02 (t, $J=7.4 \mathrm{~Hz}, 3 \mathrm{H}, \mathrm{H} 1$ ), 0.94 (t, $J=7.6 \mathrm{~Hz}, 3 \mathrm{H}, \mathrm{H} 3$ ) ppm; ${ }^{13} \mathrm{C}$ NMR (151 MHz, $\left.\mathrm{CDCl}_{3}\right): \delta=207.0$ (C11), 144.4 (C5), 141.7 (C17), 140.4 (C12), 131.2 (C15), 129.9 (C16), 128.5 (C13), 126.5 (C14), 120.8 (C6), 48.9 (C5), 34.1 (C10), 32.3 (C8), 29.2 (C2), 25.6 (C9), 23.9 (C4), 13.5 (C2), 12.9 (C1) ppm; IR (ATR): $\tilde{v}=2963(\mathrm{~m}), 2933(\mathrm{~m})$, 2874(m), 1684(s), 1599(m), 1448(m), 1372(w), 1346(w), 1276(m), 1242(w), 1217(m), 1200(m), 1160(w), 1102(w), 1073(w), 1052(w), 968(m), 921(w), 864(w), 780(m), 762(m), 738(s), 680(w) cm $\mathrm{cm}^{-1}$; HR-ESI-MS: $m / z: 265.15592\left([M+\mathrm{Na}]^{+}\right.$, calcd. for $\mathrm{C}_{17} \mathrm{H}_{22} \mathrm{ONa}^{+}$: 265.15628).

tert-Butyl 4-((5-oxo-6,7,8,9-tetrahydro-5H-benzo[7]annulen-6-yl)methylene)piperidine-1carboxylate $(( \pm)-20)$

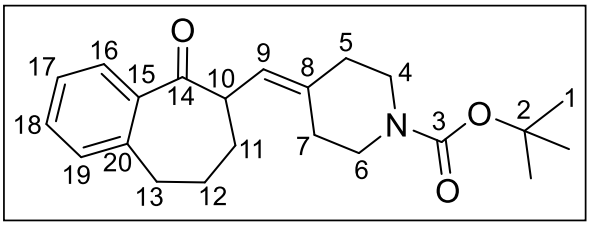

Prepared according to General Procedure B, 41 $(71.1 \mathrm{mg}, 0.20 \mathrm{mmol}, 1.00 \mathrm{eq}$.) was converted to $(\mathbf{\pm})-\mathbf{2 0}$. Purification by column chromatography $\left(\mathrm{SiO}_{2}, n-\right.$ pentane/EtOAc 9:1) yielded the product as a colorless oil.

General Procedure B: $\quad$ 1. Reaction: $(60.3 \mathrm{mg}, 0.17 \mathrm{mmol}, 85 \%)$

2. Reaction: (62.2 mg, $0.17 \mathrm{mmol}, 87 \%)$

$\mathrm{R}_{f}=0.27$ (n-pentane/EtOAc 9:1); ${ }^{1} \mathrm{H} \mathrm{NMR}\left(500 \mathrm{MHz}, \mathrm{CDCl}_{3}\right): \delta=7.60$ (dd, $J=7.7,1.5 \mathrm{~Hz}$, 1H, H16), 7.37 (tt, $J=7.4,1.1 \mathrm{~Hz}, 1 \mathrm{H}, \mathrm{H} 18), 7.26$ (td, $J=7.4,1.2 \mathrm{~Hz}, 1 \mathrm{H}, \mathrm{H} 17), 7.21$ (dd, $J=$ 7.6, 1.2 Hz, 1H, H19), 5.60 (d, $J=8.6 \mathrm{~Hz}, 1 \mathrm{H}, \mathrm{H} 9$ ), 3.75 (ddd, $J=10.5,8.7,5.5 \mathrm{~Hz}, 1 \mathrm{H}, \mathrm{H} 10$ ), $3.41(\mathrm{t}, J=5.8 \mathrm{~Hz}, 2 \mathrm{H}, \mathrm{H} 4), 3.37(\mathrm{t}, J=5.9 \mathrm{~Hz}, 2 \mathrm{H}, \mathrm{H} 6), 3.08-2.92$ (m, 2H, H13), $2.22-2.12$ (m, 4H, H5/H7), 2.12 - 2.04 (m, 1H, H12), 1.92 - 1.81 (m, 1H, H11), $1.82-1.66$ (m, 2H, H11'/H12'), 1.45 (s, 9H, H1) ppm; $\left.{ }^{13} \mathrm{C} \mathrm{NMR} \mathrm{(126} \mathrm{MHz,} \mathrm{CDCl}_{3}\right): \delta=206.1$ (C14), 154.9 (C3), 141.7 (C20), 140.0 (C15), 136.8 (C8), 131.5 (C18), 129.9 (C19), 128.6 (C16), 126.6 (C17), 122.0 (C9), 79.6 (C2), 48.2 (C10), 44.4 (b, C4/C6), 35.9 (C5), 33.8 (C13), 31.9 (C11), 29.1 (C7), 28.5 (C1), 25.4 (C12) ppm; IR (ATR): $\tilde{v}=2935(\mathrm{w}), 1862(\mathrm{w}), 1683(\mathrm{~s}), 1599(\mathrm{~m}), 1448(\mathrm{~m})$, 
1419(m), 1364(m), 1275(m), 1238(m), 1163(s), 1106(m), 990(m), 967(m), 918(w), 865(w), 767(m), 730(s), 673(w) cm ${ }^{-1}$; HR-ESI-MS: m/z: $378.20389\left([M+\mathrm{Na}]^{+}\right.$, calcd. for $\mathrm{C}_{22} \mathrm{H}_{29} \mathrm{NO}_{3} \mathrm{Na}^{+}$: 378.20396).

\section{6-((Tetrahydro-4H-pyran-4-ylidene)methyl)-6,7,8,9-tetrahydro-5H-benzo[7]annulen-5-} one $(( \pm)-21)$

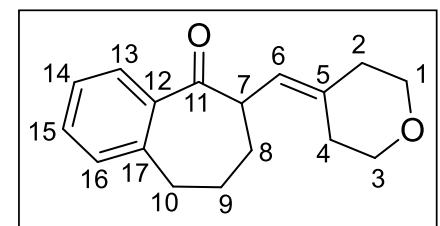

Prepared according to General Procedure B, 42 (51.2 mg, $0.20 \mathrm{mmol}, 1.00$ eq.) was converted to $( \pm)-21$. Purification by column chromatography $\left(\mathrm{SiO}_{2}, n\right.$-pentane/EtOAc 9:1) yielded the product as a colorless oil.

General Procedure B: $\quad$ 1. Reaction: (27.8 mg, $0.14 \mathrm{mmol}, 69 \%)$

2. Reaction: (24.7 mg, $0.12 \mathrm{mmol}, 62 \%)$

$\mathrm{R}_{f}=0.13\left(n\right.$-pentane/EtOAc 9:1); ${ }^{1} \mathrm{H}$ NMR $\left(500 \mathrm{MHz}, \mathrm{CDCl}_{3}\right): \delta=7.61(\mathrm{dd}, J=7.7,1.5 \mathrm{~Hz}$, 1H, H13), 7.38 (td, $J=7.5,1.5 \mathrm{~Hz}, 1 \mathrm{H}, \mathrm{H} 15), 7.29-7.25$ (m, 1H, H14), 7.21 (d, $J=7.5 \mathrm{~Hz}$, 1H, H16), 5.58 (d, $J=8.7 \mathrm{~Hz}, 1 \mathrm{H}, \mathrm{H6}$ ), 3.75 (ddd, $J=10.5,8.7,5.6 \mathrm{~Hz}, 1 \mathrm{H}, \mathrm{H} 7), 3.71$ - 3.61 (m, 4H, H1/H3), 3.07 - 2.93 (m, 2H, H10), 2.30 - 2.23 (m, 2H, H2), 2.24 - 2.20 (m, 2H, H4), $2.12-2.04$ (m, 1H, H9), $1.92-1.84$ (m, 1H, H8), 1.82 - 1.70 (m, 2H, H8'/H9') ppm; ${ }^{13} \mathrm{C} \mathrm{NMR}^{\prime}$ (126 MHz, $\left.\mathrm{CDCl}_{3}\right): \delta=206.2$ (C11), 141.7 (C17), 140.0 (C12), 136.0 (C5), 131.5 (C15), 130.0 (C16), 128.6 (C13), 126.6 (C14), 121.3 (C6), 69.6 (C1), 68.7 (C3), 48.1 (C7), 37.0 (C2), 33.9 (C10), 32.0 (C8), 30.5 (C4), 25.4 (C9) ppm; IR (ATR): $\tilde{v}=$ 2933(w), 2844(w), 1683(s), 1598(w), 1448(w), 1374(w), 1276(w), 1238(w), 1201(w), 1169(w), 1098(s), 1021(w), 998(m), 968(m), 914(w), 848(w), 784(w), 762(w), 734(m), 670(w) $\mathrm{cm}^{-1}$; HR-ESI-MS: m/z: 279.13509 $\left([M+\mathrm{Na}]^{+}\right.$, calcd. for $\left.\mathrm{C}_{17} \mathrm{H}_{20} \mathrm{O}_{2} \mathrm{Na}^{+}: 279.13555\right)$.

\section{Unsuccessful Examples:}

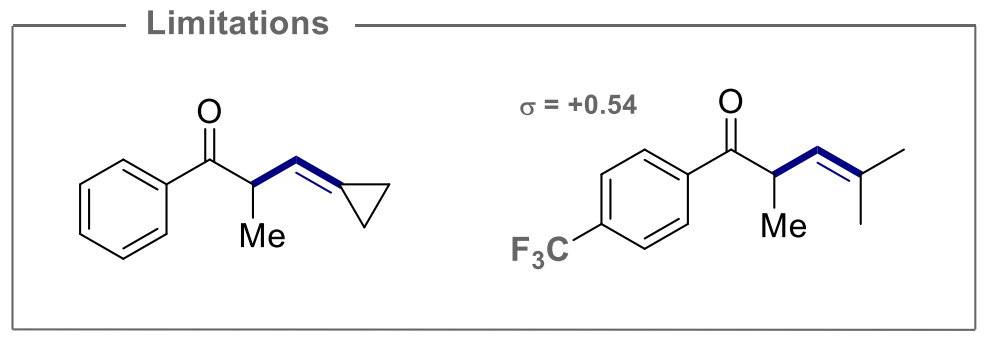


<smiles>[R]C=C([R])C(=O)c1ccc([R])cc1</smiles>

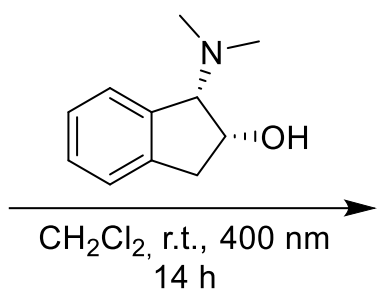<smiles>[R]C=CC([R])C(=O)C1#[R]=CC=C1</smiles>

General procedure $\mathrm{C}$ for the enantioselective photocatalytic deconjugative isomerization of acyclic alkenes (GP C)

A screw-cap vial with septum under argon was charged with the specified alkene $(0.10 \mathrm{mmol}$, 1.00 eq.) and cat. 9 (3.5 mg, $0.02 \mathrm{mmol}, 0.20$ eq.). Subsequently, degassed dichloromethane $(2 \mathrm{~mL})$ was added, the vial was sealed and irradiated at $400 \mathrm{~nm}$ for $14 \mathrm{~h}$. Afterwards, the mixture was concentrated on silica and purified by column chromatography $\left(\mathrm{SiO}_{2}, n\right.$ pentane $/ \mathrm{CH}_{2} \mathrm{Cl}_{2}$ ) to yield the products. Note: reactions were repeated, and the average was used for the Hammett correlation (vide infra).

\section{2,4-Dimethyl-1-phenylpent-3-en-1-one ((+)-2)}

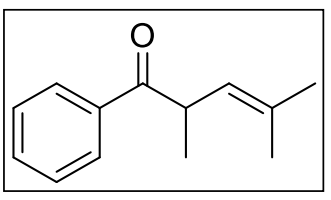

Prepared according to General procedure $\mathbf{C}$ :

1. Reaction: (14.6 mg, $0.08 \mathrm{mmol}, 78 \%)$, e.r. $64: 36$

2. Reaction: (13.1 mg, $0.07 \mathrm{mmol}, 70 \%)$, e.r. $64: 36$

$$
[\alpha]_{\mathrm{D}}^{25^{\circ} \mathrm{C}}=+50.5\left(\mathrm{c}=1.0 \text { in } \mathrm{CHCl}_{3} \text {, e.r. } 64: 36\right)
$$

HPLC (AS-H column, eluent: $n$-hexane $/ i-\mathrm{PrOH}$ 99:1, flow rate: $0.5 \mathrm{~mL} / \mathrm{min}$ ): $\mathrm{t}_{\mathrm{R}}$ (major) $=$ $8.4 \mathrm{~min} ; \mathrm{t}_{\mathrm{R}}($ minor $)=9.5 \mathrm{~min}$. 
racemic sample:

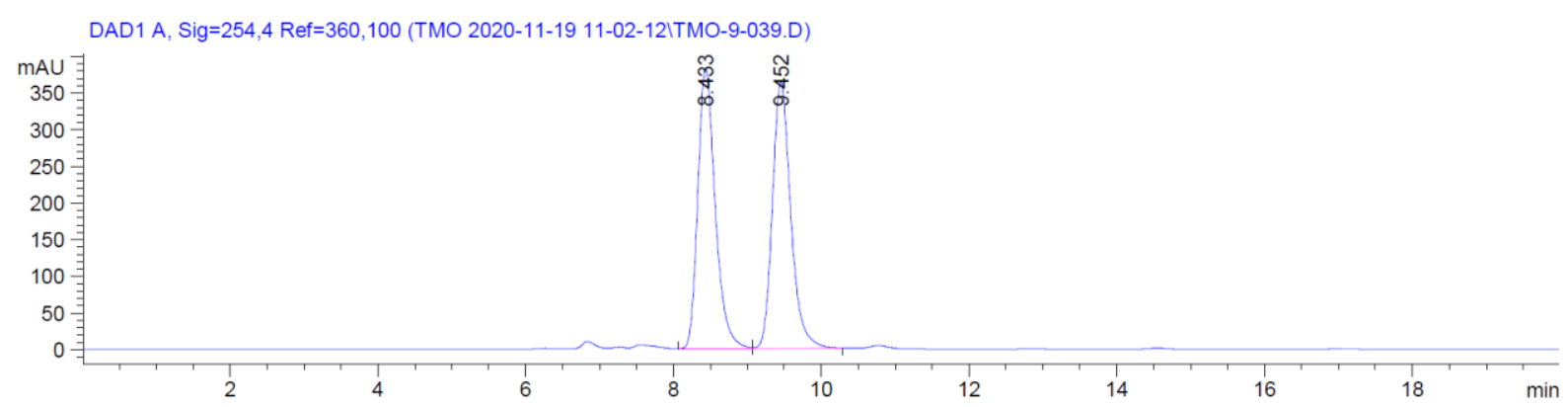

Signal 1: DAD1 A, Sig=254,4 Ref=360,100

\begin{tabular}{|c|c|c|c|c|c|c|}
\hline $\begin{array}{c}\text { Peak } \\
\#\end{array}$ & $\begin{array}{c}\text { RetTime } \\
\text { [min] }\end{array}$ & Type & $\begin{array}{l}\text { Width } \\
\text { [min] }\end{array}$ & $\begin{array}{c}\text { Area } \\
{\left[\mathrm{mAU}^{*} \mathrm{~s}\right]}\end{array}$ & $\begin{array}{l}\text { Height } \\
\text { [mAU] }\end{array}$ & $\begin{array}{c}\text { Area } \\
\frac{\circ}{0}\end{array}$ \\
\hline 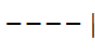 & & & & $1-$ & --- & \\
\hline 1 & 8.43 & & & 6282.85254 & 383.33203 & 446 \\
\hline 2 & 9.452 & & 556 & 6296.79834 & 364.37964 & 0554 \\
\hline
\end{tabular}

enantioselective sample:

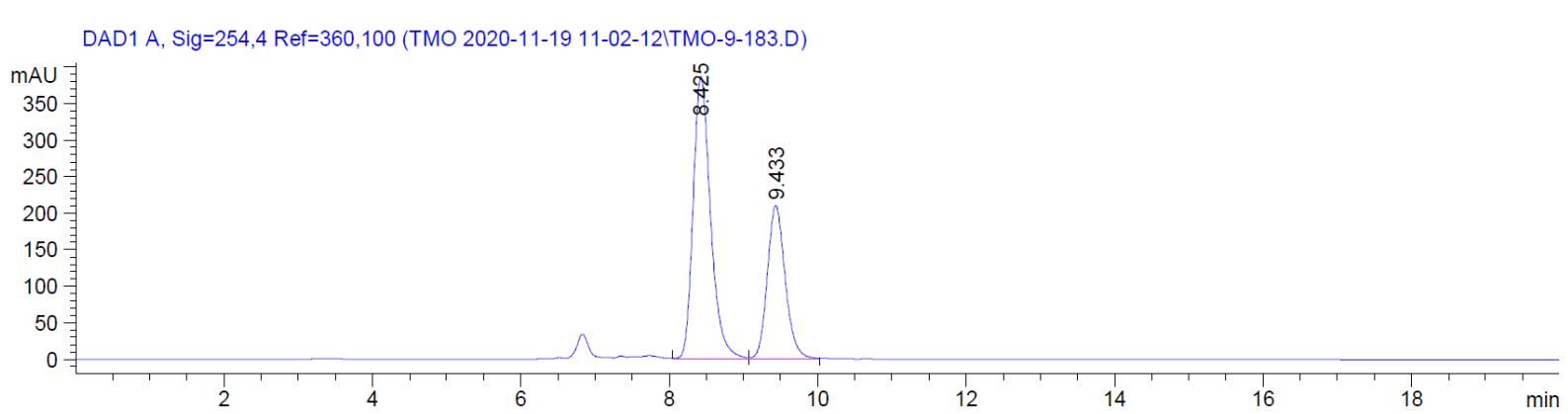

Signal 1: DAD1 A, Sig=254,4 Ref=360,100

\begin{tabular}{|c|c|c|c|c|c|c|}
\hline $\begin{array}{l}\text { eak } \\
\#\end{array}$ & $\begin{array}{c}\text { RetTime } \\
\text { [min] }\end{array}$ & Type & $\begin{array}{l}\text { Width } \\
\text { [min] }\end{array}$ & $\begin{array}{c}\text { Area } \\
{\left[\mathrm{mAU}^{\star} \mathrm{s}\right]}\end{array}$ & $\begin{array}{l}\text { Height } \\
{[\mathrm{mAU}]}\end{array}$ & $\begin{array}{c}\text { Area } \\
\frac{\circ}{\circ}\end{array}$ \\
\hline & 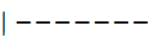 & & & --- & --- & --1 \\
\hline & & & & 2979 & 385.96964 & 39 \\
\hline & 8 & & 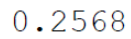 & 1538 & 209.45119 & 6961 \\
\hline
\end{tabular}

\section{1-(4-Methoxyphenyl)-2,4-dimethylpent-3-en-1-one ((+)-3)}

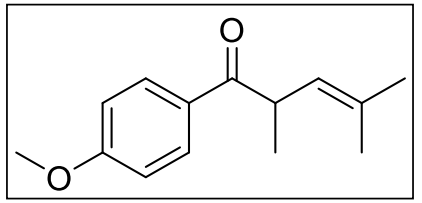

Prepared according to General procedure $\mathbf{C}$ :

1. Reaction: (21.8 mg, $0.10 \mathrm{mmol}$, quant.), e.r. $70: 30$

2. Reaction: (21.9 mg, $0.10 \mathrm{mmol}$, quant.), e.r. $68: 32$

$[\alpha]_{\mathrm{D}}^{25^{\circ} \mathrm{C}}=+58.6\left(\mathrm{c}=1.0\right.$ in $\mathrm{CHCl}_{3}$, e.r. $\left.70: 30\right)$ 
HPLC (AS-H column, eluent: $n$-hexane/ $i$-PrOH 99:1, flow rate: $0.5 \mathrm{~mL} / \mathrm{min}$ ): $\mathrm{t}_{\mathrm{R}}$ (major) $=$ $15.0 \mathrm{~min} ; \mathrm{t}_{\mathrm{R}}($ minor $)=19.3 \mathrm{~min}$.

racemic sample:

DAD1 A, Sig=254,4 Ref=360, 100 (TMO 2020-11-24 15-50-45।TMO-9-060.D)

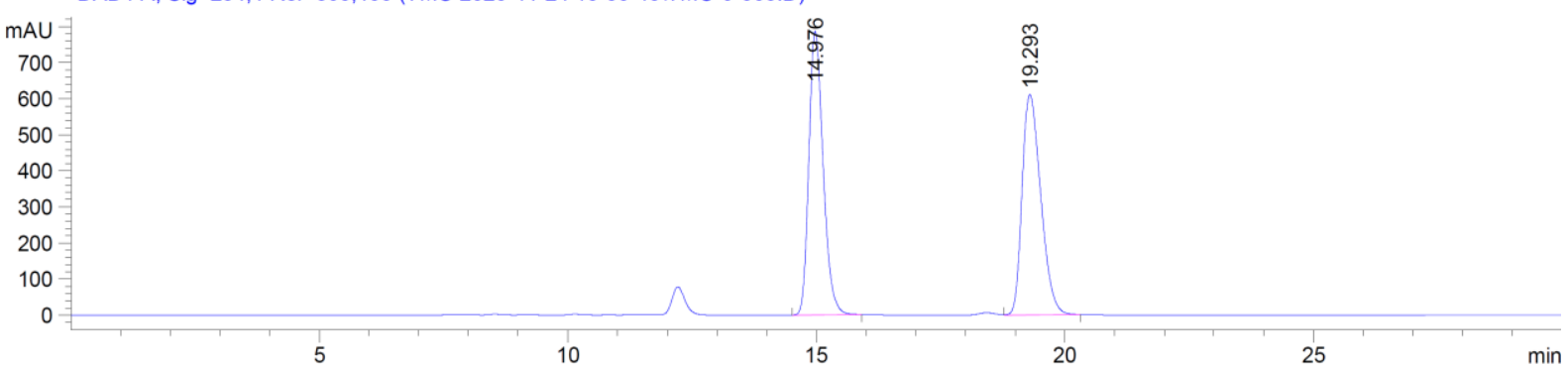

Signal 1: DAD1 A, Sig=254,4 Ref=360,100

\begin{tabular}{|c|c|c|c|c|c|c|}
\hline $\begin{array}{c}\text { Peak } \\
\quad \#\end{array}$ & $\begin{array}{c}\text { RetTime } \\
\text { [min] }\end{array}$ & Type & $\begin{array}{l}\text { Width } \\
\text { [min] }\end{array}$ & $\begin{array}{c}\text { Area } \\
{\left[\mathrm{mAU}{ }^{\star} \mathrm{s}\right]}\end{array}$ & $\begin{array}{l}\text { Height } \\
\text { [mAU] }\end{array}$ & $\begin{array}{c}\text { Area } \\
\quad \%\end{array}$ \\
\hline & & & 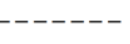 & | --------- & ---- & -------- \\
\hline 1 & 76 & $\mathrm{BB}$ & 12 & $1.58555 \mathrm{e} 4$ & 786.36292 & 49.9583 \\
\hline 2 & 19.293 & VB & 4052 & $1.58820 \mathrm{e} 4$ & 611.10156 & 50.0417 \\
\hline
\end{tabular}

enantioselective sample:

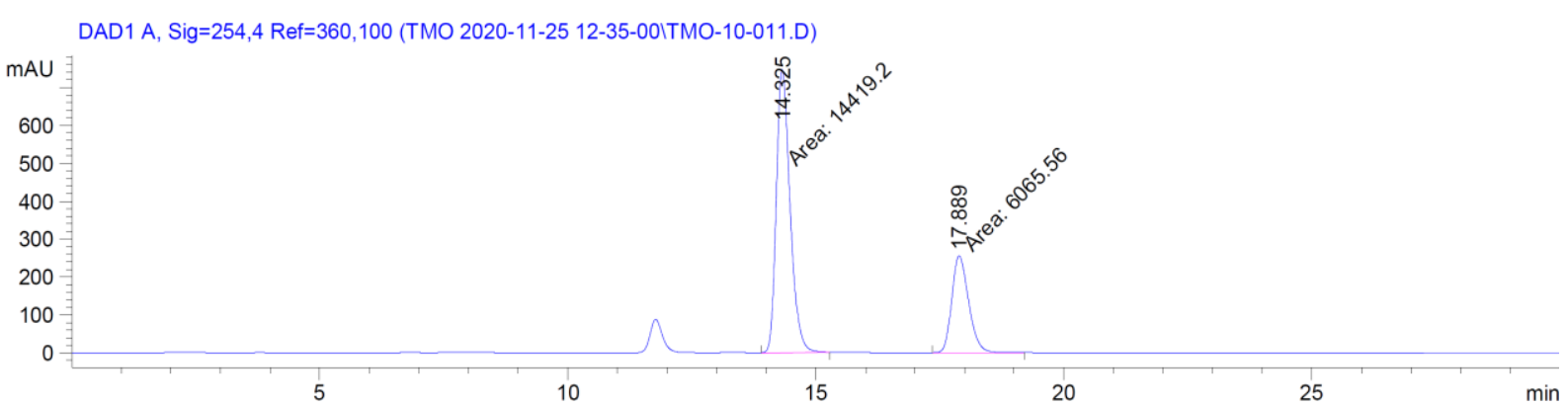

Signal 1: DAD1 A, Sig=254, 4 Ref=360,100

\begin{tabular}{|c|c|c|c|c|c|c|}
\hline $\begin{array}{c}\text { Peak } \\
\#\end{array}$ & $\begin{array}{c}\text { RetTime } \\
\text { [min] }\end{array}$ & Type & $\begin{array}{l}\text { Width } \\
\text { [min] }\end{array}$ & $\begin{array}{c}\text { Area } \\
{\left[\mathrm{mAU}^{\star} \mathrm{S}\right]}\end{array}$ & $\begin{array}{l}\text { Height } \\
{[\mathrm{mAU}]}\end{array}$ & $\begin{array}{c}\text { Area } \\
\quad \%\end{array}$ \\
\hline & & & & & & \\
\hline 1 & .32 & $M$ & 3 & $1.44192 \mathrm{e} 4$ & 75446 & 899 \\
\hline 2 & 17.889 & & & 6065.55664 & .46085 & 101 \\
\hline
\end{tabular}

1-(4-Methylphenyl)-2,4-dimethylpent-3-en-1-one ((+)-4)

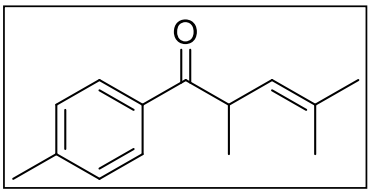

Prepared according to General procedure $\mathbf{C}$ :

1. Reaction: (16.8 mg, $0.08 \mathrm{mmol}, 83 \%)$, e.r. $58: 42$

2. Reaction: (14.8 mg, $0.10 \mathrm{mmol}, 73 \%)$, e.r. $58: 42$

$[\alpha]_{\mathrm{D}}^{25^{\circ} \mathrm{C}}=+28.4\left(\mathrm{c}=1.0\right.$ in $\mathrm{CHCl}_{3}$, e.r. $\left.58: 42\right)$ 
HPLC (AS-H column, eluent: $n$-hexane $/ i-\mathrm{PrOH}$ 99:1, flow rate: $0.5 \mathrm{~mL} / \mathrm{min}$ ): $\mathrm{t}_{\mathrm{R}}$ (major) $=$ $8.9 \mathrm{~min} ; \mathrm{t}_{\mathrm{R}}($ minor $)=10.9 \min$.

racemic sample:

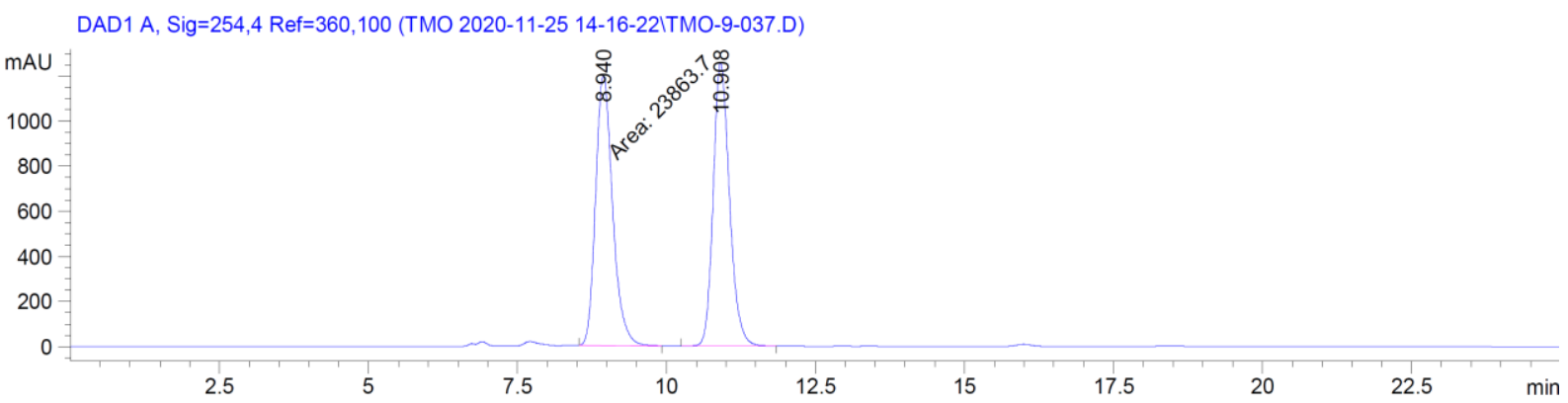

Signal 1: DAD1 A, Sig=254,4 Ref=360, 100

\begin{tabular}{|c|c|c|c|c|c|c|}
\hline $\begin{array}{c}\text { Peak } \\
\text { \# }\end{array}$ & $\begin{array}{c}\text { RetTime } \\
\text { [min] }\end{array}$ & Type & $\begin{array}{l}\text { Width } \\
\text { [min] }\end{array}$ & $\begin{array}{c}\text { Area } \\
{\left[\mathrm{mAU}^{\star} \mathrm{s}\right]}\end{array}$ & $\begin{array}{l}\text { Height } \\
\text { [mAU] }\end{array}$ & $\begin{array}{c}\text { Area } \\
\frac{\circ}{0}\end{array}$ \\
\hline & & & & & & \\
\hline 1 & & IM & & 2.38 & 11 & 49 \\
\hline - & 10 . & $2 \mathrm{R}$ & & $2.38773 e 4$ & 198 & 50 \\
\hline
\end{tabular}

enantioselective sample:

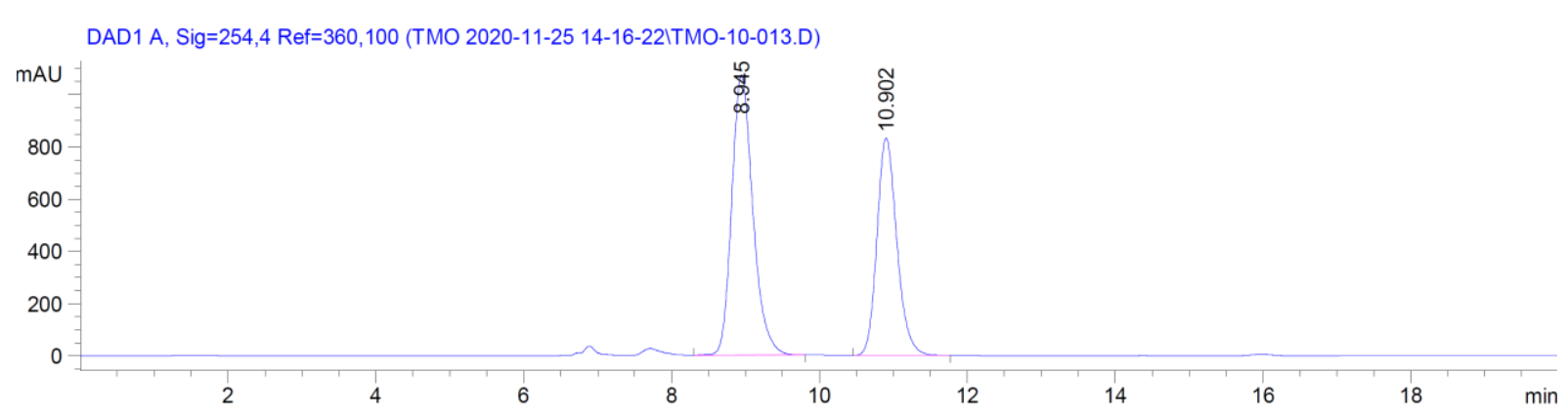

Signal 1: DAD1 A, Sig=254,4 Ref=360,100

\begin{tabular}{|c|c|c|c|c|c|c|}
\hline $\begin{array}{c}\text { Peak } \\
\text { \# }\end{array}$ & $\begin{array}{c}\text { RetTime } \\
\text { [min] }\end{array}$ & Type & $\begin{array}{l}\text { Width } \\
\text { [min] }\end{array}$ & $\begin{array}{c}\text { Area } \\
{\left[\mathrm{mAU}{ }^{*} \mathrm{~S}\right]}\end{array}$ & $\begin{array}{l}\text { Height } \\
\text { [mAU] }\end{array}$ & $\begin{array}{c}\text { Area } \\
\frac{\circ}{0}\end{array}$ \\
\hline & & & & --- & $|----------|$ & $--------\mid$ \\
\hline 1 & 9 & $\mathrm{BB}$ & 63 & $2.13623 e 4$ & 1072.87048 & 57.8581 \\
\hline 2 & 10.902 & $\mathrm{BB}$ & 0.2883 & $1.55596 \mathrm{e} 4$ & 831.38062 & 42.1419 \\
\hline
\end{tabular}

\section{1-(4-Fluorophenyl)-2,4-dimethylpent-3-en-1-one ((+)-5)}

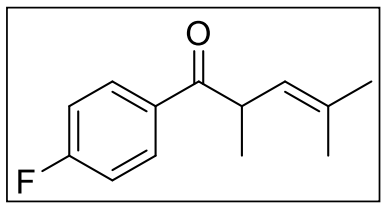

Prepared according to General procedure $\mathbf{C}$ :

1. Reaction: (14.3 mg, $0.07 \mathrm{mmol}, 69 \%)$, e.r. 59:41

2. Reaction: (16.9 mg, $0.08 \mathrm{mmol}, 82 \%)$, e.r. $58: 42$

$[\alpha]_{\mathrm{D}}^{25^{\circ} \mathrm{C}}=+29.5\left(\mathrm{c}=1.0\right.$ in $\mathrm{CHCl}_{3}$, e.r. $\left.58: 42\right)$ 
HPLC (AS-H column, eluent: $n$-hexane $/ i-\mathrm{PrOH}$ 99:1, flow rate: $0.5 \mathrm{~mL} / \mathrm{min}$ ): $\mathrm{t}_{\mathrm{R}}$ (major) $=$ $8.3 \mathrm{~min} ; \mathrm{t}_{\mathrm{R}}($ minor $)=9.6 \mathrm{~min}$.

racemic sample:

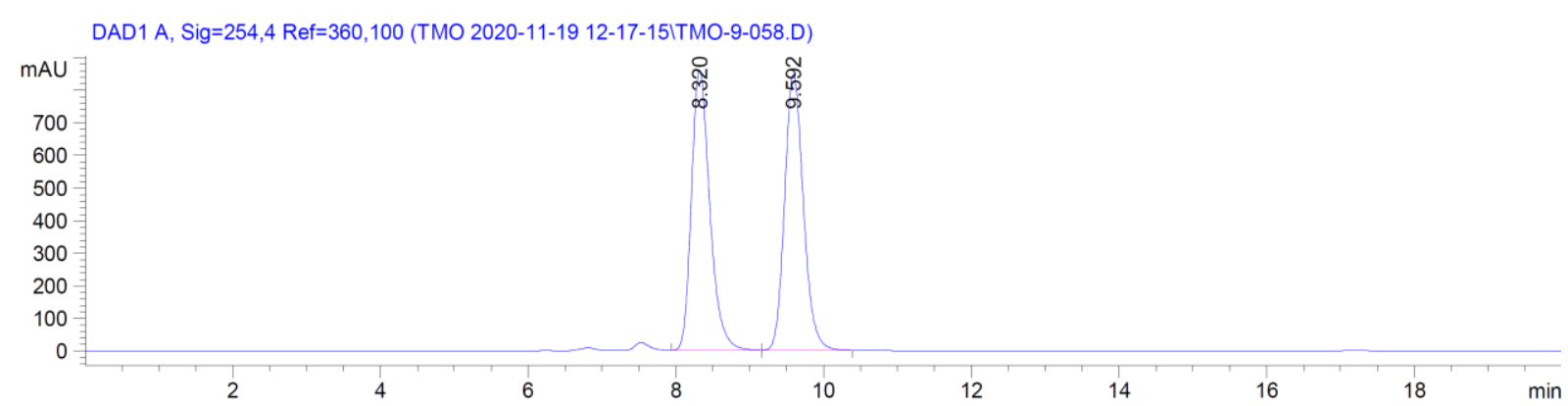

Signal 1: DAD1 A, Sig=254,4 $\operatorname{Ref}=360,100$

\begin{tabular}{|c|c|c|c|c|c|c|}
\hline $\begin{array}{c}\text { Peak } \\
\#\end{array}$ & $\begin{array}{c}\text { RetTime } \\
\text { [min] }\end{array}$ & Type & $\begin{array}{l}\text { Width } \\
\text { [min] }\end{array}$ & $\begin{array}{c}\text { Area } \\
{\left[\mathrm{mAU}^{\star} \mathrm{s}\right]}\end{array}$ & $\begin{array}{l}\text { Height } \\
{[\mathrm{mAU}]}\end{array}$ & $\begin{array}{c}\text { Area } \\
\quad \%\end{array}$ \\
\hline & & & & & $=--1$ & - \\
\hline 1 & & & & $5 e 4$ & 8987 & 598 \\
\hline 2 & & & & 1.49 & 3605 & 402 \\
\hline
\end{tabular}

enantioselective sample:

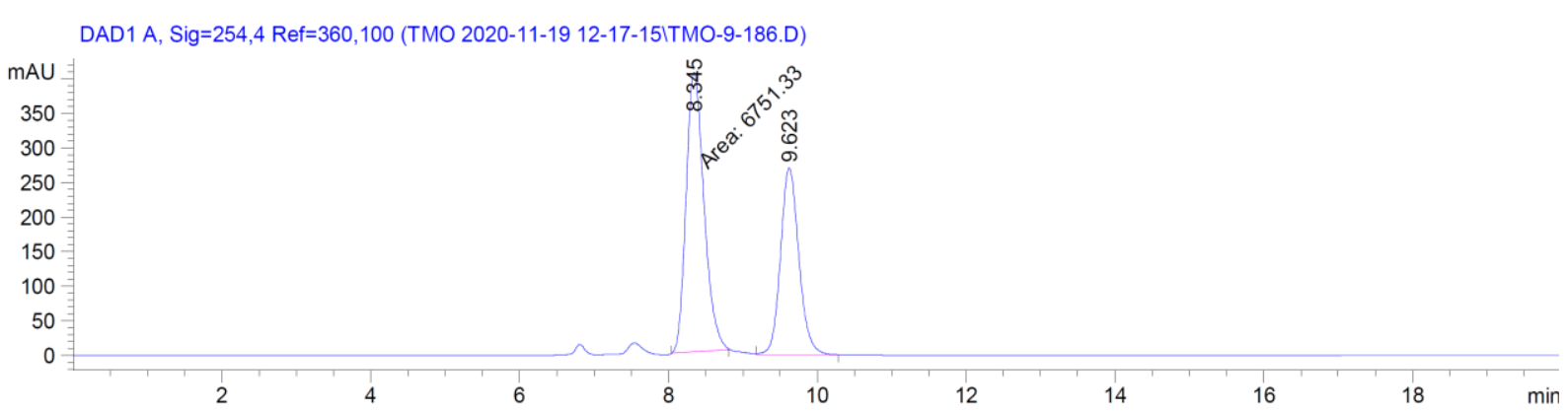

Signal 1: DAD1 A, Sig=254,4 Ref=360,100

\begin{tabular}{|c|c|c|c|c|c|c|}
\hline $\begin{array}{c}\text { Peak } \\
\text { \# }\end{array}$ & $\begin{array}{c}\text { RetTime } \\
\text { [min] }\end{array}$ & Type & $\begin{array}{l}\text { Width } \\
\text { [min] }\end{array}$ & $\begin{array}{c}\text { Area } \\
{\left[\mathrm{mAU}{ }^{\star} \mathrm{s}\right]}\end{array}$ & $\begin{array}{l}\text { Height } \\
\text { [mAU] }\end{array}$ & $\begin{array}{c}\text { Area } \\
\frac{\circ}{0}\end{array}$ \\
\hline & & & & ------- & . & . \\
\hline 1 & & & & 675 & 442 & 50 \\
\hline 2 & & & & 97510 & 1655 & 7690 \\
\hline
\end{tabular}

\section{1-(4-Bromophenyl)-2,4-dimethylpent-3-en-1-one ((+)-6)}

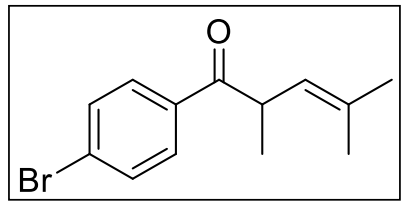

Prepared according to General procedure $\mathbf{C}$ :

1. Reaction: (12.4 mg, $0.05 \mathrm{mmol}, 46 \%)$, e.r. $54: 46$

2. Reaction: (11.1 mg, $0.04 \mathrm{mmol}, 42 \%)$, e.r. $54: 46$

$[\alpha]_{\mathrm{D}}^{25^{\circ} \mathrm{C}}=+10.8\left(\mathrm{c}=1.0\right.$ in $\mathrm{CHCl}_{3}$, e.r. $\left.54: 46\right)$ 
HPLC (AS-H column, eluent: $n$-hexane $/ i-\mathrm{PrOH}$ 99:1, flow rate: $0.5 \mathrm{~mL} / \mathrm{min}$ ): $\mathrm{t}_{\mathrm{R}}$ (major) $=$ $8.6 \mathrm{~min} ; \mathrm{t}_{\mathrm{R}}($ minor $)=10.2 \mathrm{~min}$.

racemic sample:

DAD1 A, Sig=254,4 Ref=360,100 (TMO 2020-11-20 16-17-27\TMO-9-068.D)

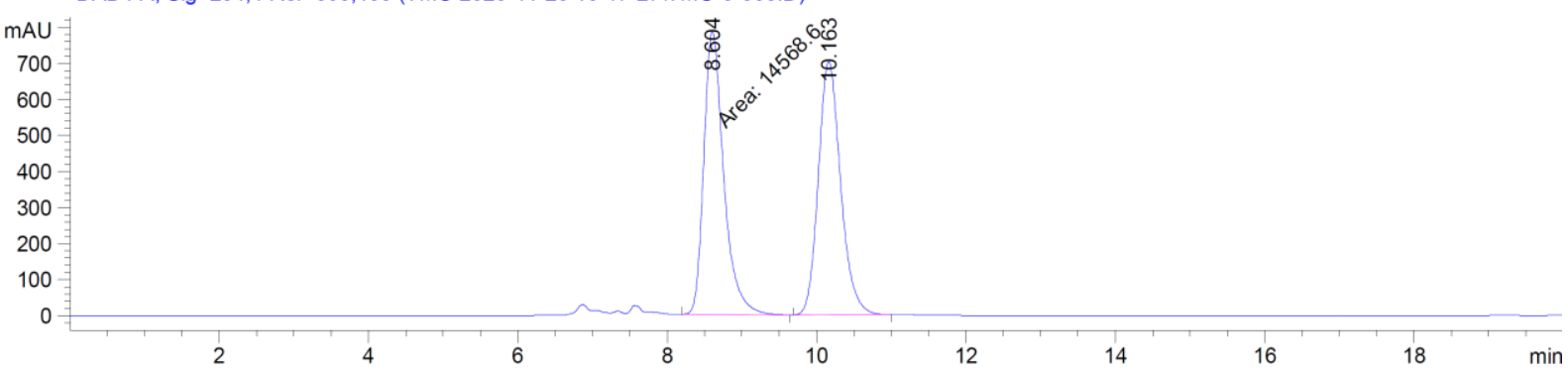

Signal 1: DAD1 A, Sig=254,4 Ref=360,100

\begin{tabular}{|c|c|c|c|c|c|c|}
\hline $\begin{array}{c}\text { Peak } \\
\#\end{array}$ & $\begin{array}{c}\text { RetTime } \\
\text { [min] }\end{array}$ & Type & $\begin{array}{l}\text { Width } \\
\text { [min] }\end{array}$ & $\begin{array}{c}\text { Area } \\
{\left[\mathrm{mAU}{ }^{\star} \mathrm{s}\right]}\end{array}$ & $\begin{array}{l}\text { Height } \\
{[\mathrm{mAU}]}\end{array}$ & $\begin{array}{c}\text { Area } \\
\frac{\circ}{\circ}\end{array}$ \\
\hline & & & & ---- & --- & --1 \\
\hline 1 & 8.604 & MM & 089 & $1.45686 \mathrm{e} 4$ & 786.01007 & 021 \\
\hline 2 & 0.163 & $\mathrm{BB}$ & 80 & $1.45674 \mathrm{e} 4$ & 702.32471 & 49.9979 \\
\hline
\end{tabular}

enantioselective sample:

DAD1 A, Sig=254,4 Ref=360,100 (TMO 2020-11-20 16-17-27ıTMO-10-002.D)

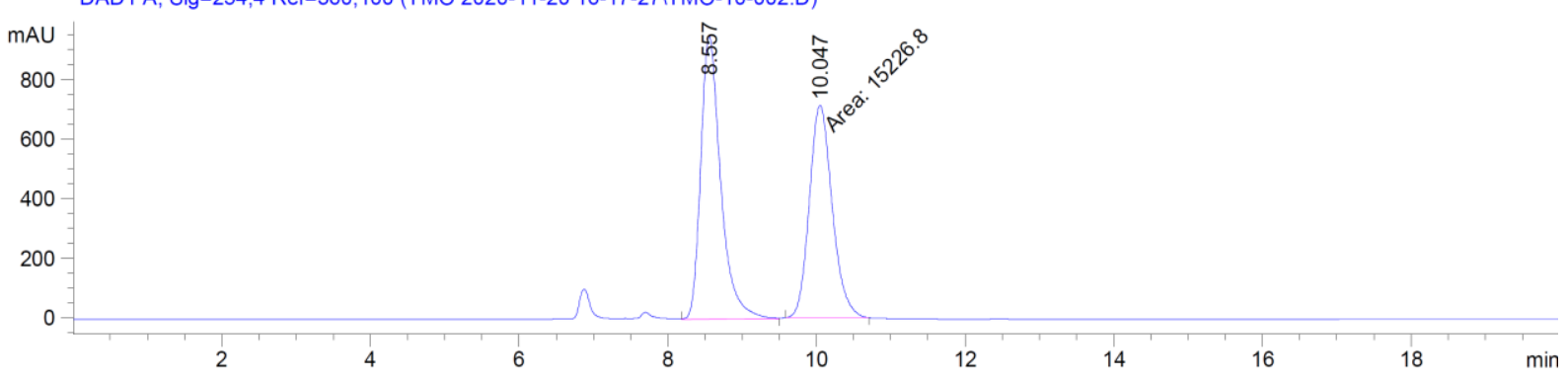

Signal 1: DAD1 A, Sig=254,4 Ref=360,100

\begin{tabular}{|c|c|c|c|c|c|c|}
\hline Peak & RetTime & Type & Width & Area & Height & Area \\
\hline$\#$ & [min] & & [min] & {$\left[\mathrm{mAU}{ }^{*} \mathrm{~s}\right]$} & {$[\mathrm{mAU}]$} & $\%$ \\
\hline & - & --1 & 1 & ---------- & $----------\mid$ & $--------\mid$ \\
\hline 1 & 8.557 & $\mathrm{BB}$ & 0.2823 & $1.78212 \mathrm{e} 4$ & 952.25897 & 53.9253 \\
\hline 2 & 10.047 & $\mathrm{MM}$ & 0.3555 & $1.52267 e 4$ & 713.78241 & 46.0747 \\
\hline
\end{tabular}

\section{Enantioselective deconjugative isomerization of cyclic alkenes}<smiles>[R]C=C1CCCCC1=O</smiles>

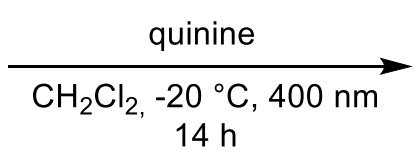<smiles>[R]C=C1CCCCC1=O</smiles> 
General procedure D for the enantioselective photocatalytic deconjugative isomerization of cyclic alkenes

A screw-cap vial with septum under argon was charged with the specified alkene $(0.20 \mathrm{mmol}$, 1.00 eq.) and quinine (13.0 mg, $0.04 \mathrm{mmol}, 0.20$ eq.). Subsequently, degassed dichloromethane $(4 \mathrm{~mL})$ was added, the vial was sealed and the septum was perforated to insert a quartz glass rod. The reaction mixture was cooled to $-20{ }^{\circ} \mathrm{C}$ and irradiated at $400 \mathrm{~nm}$ for $14 \mathrm{~h}$. Afterwards, the mixture was warmed to r.t., concentrated on silica and purified by column chromatography $\left(\mathrm{SiO}_{2}, n\right.$-pentane/ $\mathrm{CH}_{2} \mathrm{Cl}_{2}$ ) to yield the products. Note: reactions for scope entries were repeated and yields and e.r. given in the paper are the average of two independent experiments.

\section{Optimization of the enantioselective deconjugative isomerization of cyclic-alkenes}<smiles>CC(C)C=C1CCc2ccccc2C1=O</smiles>

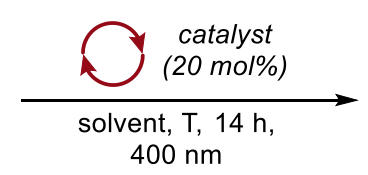<smiles>CC(C)=CC1CCc2ccccc2C1=O</smiles><smiles>CN(C)[C@H]1c2ccccc2C[C@H]1O</smiles>

cat. 9<smiles>[In]NCC1CCCN1</smiles>

cat. 10<smiles>OC(c1cc(C(F)(F)F)cc(C(F)(F)F)c1)(c1cc(C(F)(F)F)cc(C(F)(F)F)c1)C1CCCN1</smiles>

cat. 11<smiles>C=CC1=CN2CCC(CC2)[C@H]1C(O)c1ccnc2ccc(OC)cc12</smiles>

cat. 12

Table S3: Optimization of the enantioselective deconjugative isomerization of alkenes.

\begin{tabular}{ccccccc}
\hline Entry & Solvent & Catalyst & $\mathbf{T}\left[{ }^{\circ} \mathrm{C}\right]$ & Yield & e.r. & Comment \\
\hline \hline 1 & $\mathrm{CH}_{2} \mathrm{Cl}_{2}$ & 9 & 25 & $59 \%$ & $38 / 62$ & \\
2 & $\mathrm{CH}_{2} \mathrm{Cl}_{2}$ & 10 & 25 & $62 \%$ & $39 / 61$ & \\
3 & $\mathrm{CH}_{2} \mathrm{Cl}_{2}$ & 11 & 25 & $57 \%$ & $37 / 63$ & \\
4 & $\mathrm{CH}_{2} \mathrm{Cl}_{2}$ & 12 & 25 & $68 \%$ & $75 / 25$ & \\
5 & $\mathrm{CH}_{2} \mathrm{Cl}_{2}$ & 12 & 25 & $70 \%$ & $72 / 28$ & $3 \AA \AA \mathrm{MS}$ \\
6 & $\mathrm{CH}_{2} \mathrm{Cl}_{2}$ & 12 & 25 & $63 \%$ & $72 / 28$ & 0.2 eq $\mathrm{H}_{2} \mathrm{O}$ \\
7 & $\mathrm{CH}_{2} \mathrm{Cl}_{2}$ & 12 & 25 & $65 \%$ & $72 / 28$ & 1.0 eq $\mathrm{H}_{2} \mathrm{O}$ \\
8 & $\mathrm{CH}_{2} \mathrm{Cl}_{2}$ & 12 & 0 & $87 \%$ & $85 / 15$ & \\
9 & $\mathrm{CH}_{2} \mathrm{Cl}_{2}$ & 12 & -20 & $75 \%$ & $89 / 11$ & \\
10 & $\mathrm{CH}_{2} \mathrm{Cl}_{2}$ & 12 & -40 & $82 \%$ & $85 / 15$ & Precipitation \\
11 & $\mathrm{CH}_{2} \mathrm{Cl}_{2}$ & 12 & -65 & $56 \%$ & $85 / 15$ & Precipitation \\
\hline
\end{tabular}

${ }^{[a]}$ All reactions perfomed on a $0.1 \mathrm{mmol}$ scale. 


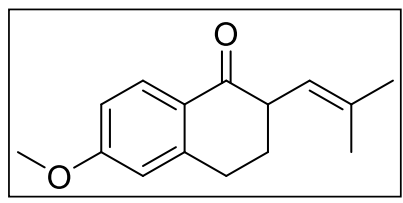

Prepared according to General procedure D:

1. Reaction: (40.8 mg, $0.18 \mathrm{mmol}, 89 \%)$, e.r. $93: 7$

2. Reaction: (39.9 mg, $0.17 \mathrm{mmol}, 87 \%)$, e.r. $93: 7$

$[\alpha]_{\mathrm{D}}^{25^{\circ} \mathrm{C}}=-37.8\left(\mathrm{c}=1.0\right.$ in $\mathrm{CHCl}_{3}$, e.r. $\left.93: 7\right)$

The enantiomer (+)-11 can be obtained using quinidine as catalyst:

1. Reaction: (43.6 mg, $0.19 \mathrm{mmol}, 95 \%)$, e.r. $8: 92$

2. Reaction: (45.5 mg, $0.20 \mathrm{mmol}, 98 \%)$, e.r. 8:92

HPLC (AS-H column, eluent: $n$-hexane $/ i$-PrOH 95:5, flow rate: $0.5 \mathrm{~mL} / \mathrm{min}$ ): $\mathrm{t}_{\mathrm{R}}$ (major) $=$ $17.3 \mathrm{~min} ; \mathrm{t}_{\mathrm{R}}($ minor $)=27.5 \mathrm{~min}$.

racemic sample:

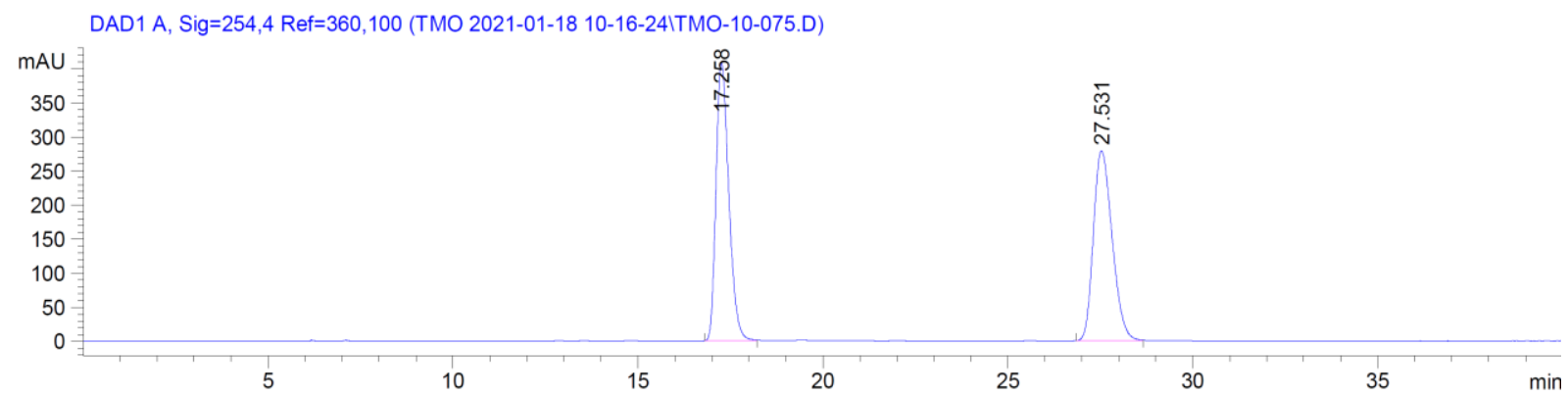

Signal 1: DAD1 A, Sig=254,4 Ref=360,100

\begin{tabular}{|c|c|c|c|c|c|c|}
\hline $\begin{array}{c}\text { Peak } \\
\text { \# }\end{array}$ & $\begin{array}{c}\text { RetTime } \\
\text { [min] }\end{array}$ & Type & $\begin{array}{l}\text { Width } \\
{[\mathrm{min}]}\end{array}$ & $\begin{array}{c}\text { Area } \\
{\left[\mathrm{mAU}{ }^{\star} \mathrm{S}\right]}\end{array}$ & $\begin{array}{l}\text { Height } \\
\text { [mAU] }\end{array}$ & $\begin{array}{c}\text { Area } \\
\frac{\circ}{0}\end{array}$ \\
\hline & & & & --- & ---- & $------\mid$ \\
\hline 1 & 7 & B & & 9801.31934 & 409.43231 & 49.9316 \\
\hline 2 & 27.531 & BB & 31 & 9828.17578 & 278.93649 & 684 \\
\hline
\end{tabular}

enantioselective sample: (-)-11

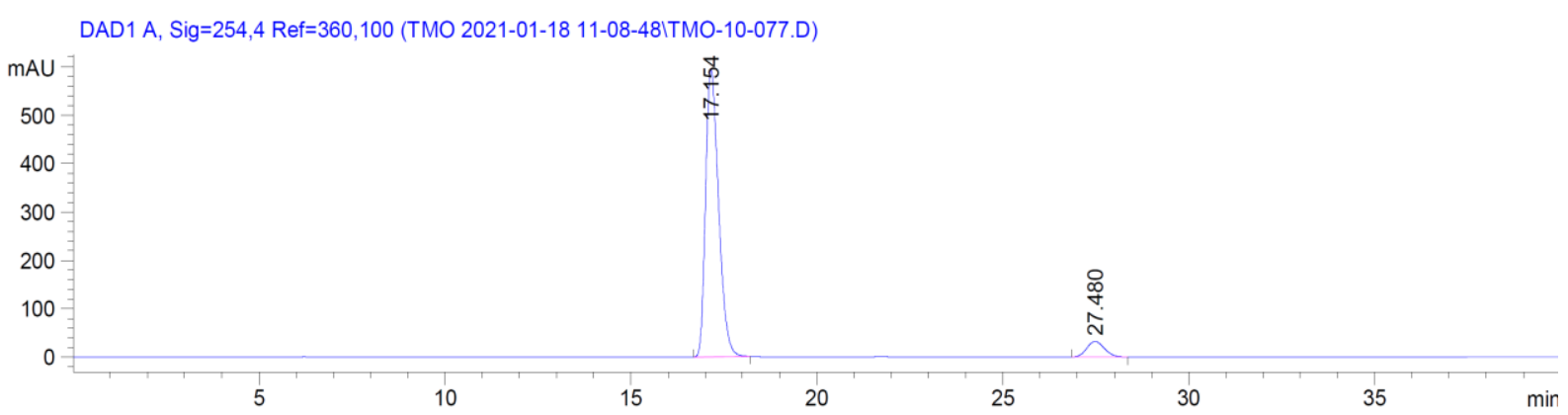


Signal 1: DAD1 A, Sig=254,4 Ref=360,100

\begin{tabular}{|c|c|c|c|c|c|c|}
\hline $\begin{array}{c}\text { Peak } \\
\#\end{array}$ & $\begin{array}{c}\text { RetTime } \\
\text { [min] }\end{array}$ & Type & $\begin{array}{c}\text { Width } \\
\text { [min] }\end{array}$ & $\begin{array}{c}\text { Area } \\
{\left[\mathrm{mAU}^{*} \mathrm{~s}\right]}\end{array}$ & $\begin{array}{l}\text { Height } \\
{[\mathrm{mAU}]}\end{array}$ & $\begin{array}{c}\text { Area } \\
\frac{\circ}{0}\end{array}$ \\
\hline & & & & --- & - & --- \\
\hline 1 & 1 & $\mathrm{BB}$ & 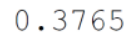 & $1.44153 \mathrm{e} 4$ & 595.24872 & 9744 \\
\hline 2 & 80 & R & 192 & 1089.28735 & 32.64888 & 7.0256 \\
\hline
\end{tabular}

enantioselective sample: $(+)-11$
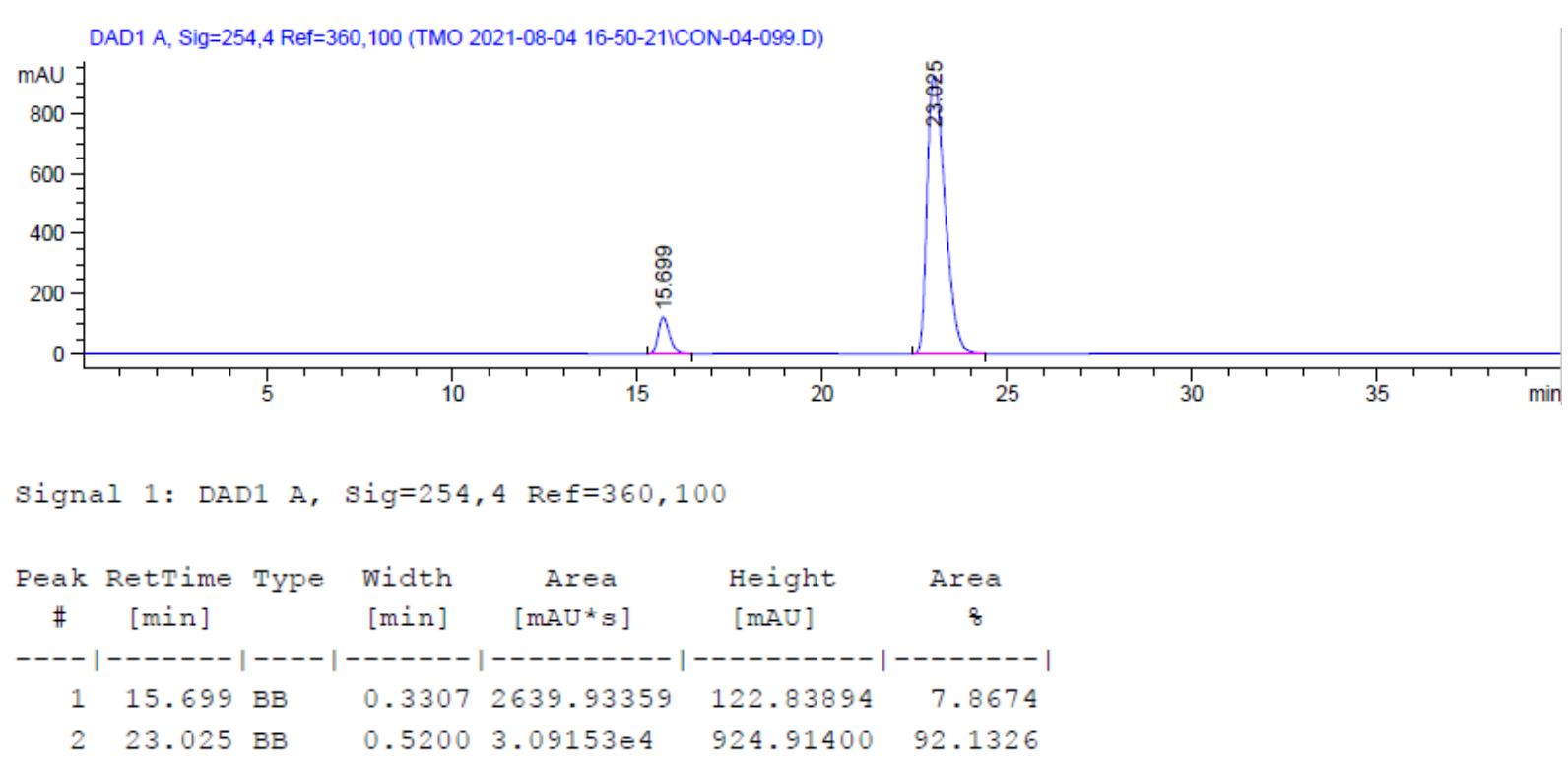

\section{2-(2-Methylprop-1-en-1-yl)-3,4-dihydronaphthalen-1(2H)-one ((-)-12)}

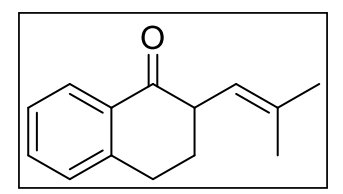

Prepared according to General procedure D:

1. Reaction: (33.2 mg, $0.17 \mathrm{mmol}, 83 \%)$, e.r. $92: 8$

2. Reaction: (33.1 mg, $0.17 \mathrm{mmol}, 83 \%)$, e.r. $92: 8$

$$
[\alpha]_{\mathrm{D}}^{25^{\circ} \mathrm{C}}=-82.5\left(\mathrm{c}=1.0 \text { in } \mathrm{CHCl}_{3} \text {, e.r. } 92: 8\right)
$$

HPLC (AS-H column, eluent: $n$-hexane $/ i$-PrOH 99.8:0.2, flow rate: $0.5 \mathrm{~mL} / \mathrm{min}): \mathrm{t}_{\mathrm{R}}$ (major) $=$ $14.7 \mathrm{~min} ; \mathrm{t}_{\mathrm{R}}(\operatorname{minor})=21.9 \mathrm{~min}$.

racemic sample: 

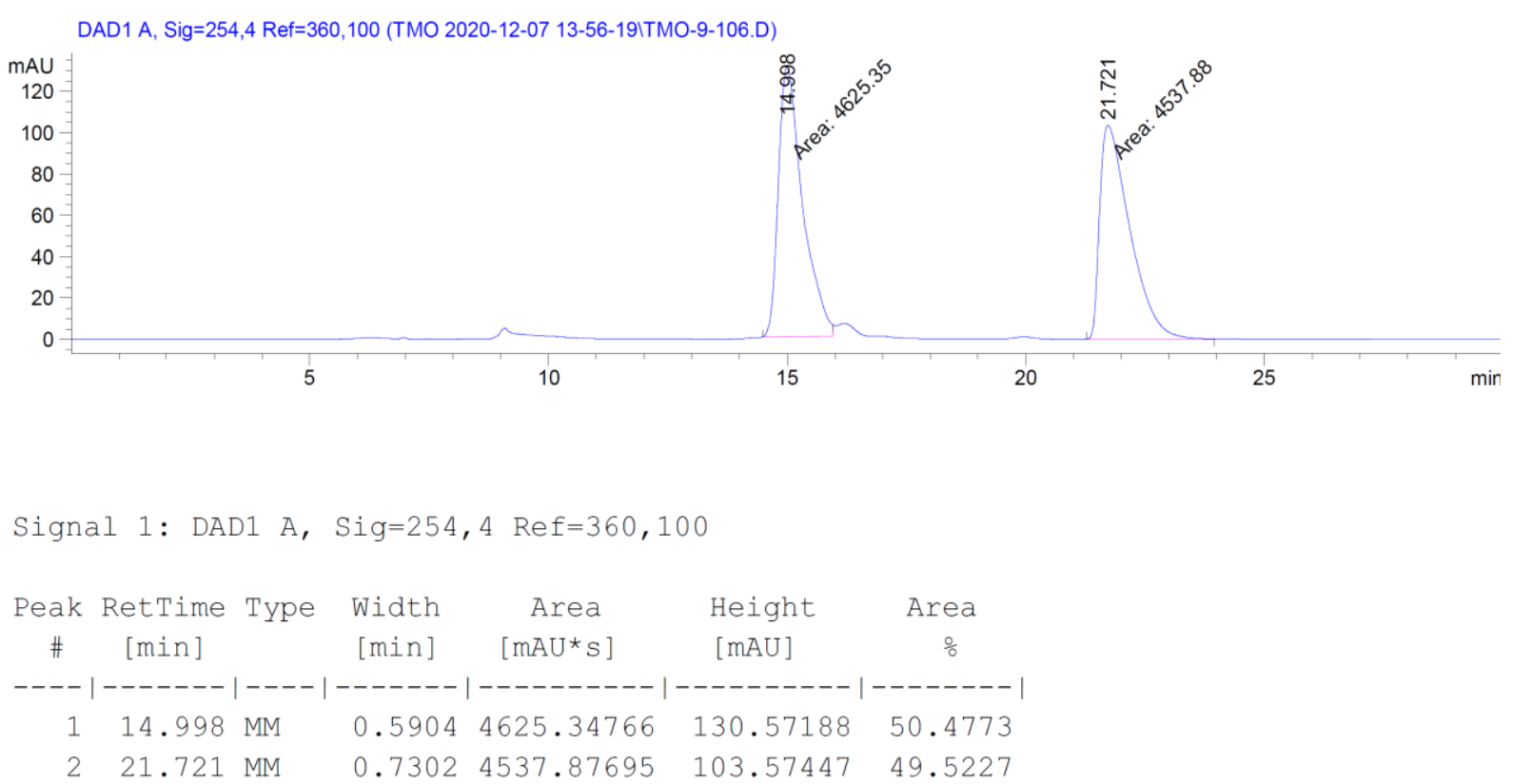

enantioselective sample:

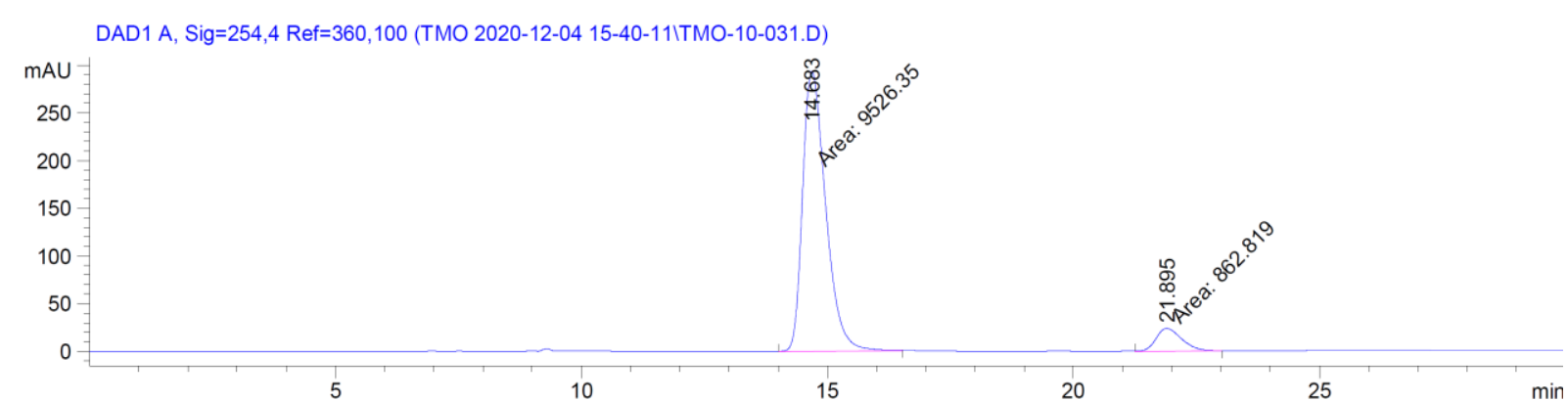

Signal 1: DAD1 A, Sig=254,4 Ref=360,100

\begin{tabular}{|c|c|c|c|c|c|c|}
\hline $\begin{array}{c}\text { Peak } \\
\text { \# }\end{array}$ & $\begin{array}{c}\text { RetTime } \\
\text { [min] }\end{array}$ & Type & $\begin{array}{l}\text { Width } \\
\text { [min] }\end{array}$ & $\begin{array}{c}\text { Area } \\
{\left[\mathrm{mAU}^{\star} \mathrm{S}\right]}\end{array}$ & $\begin{array}{l}\text { Height } \\
\text { [mAU] }\end{array}$ & $\begin{array}{c}\text { Area } \\
\frac{\circ}{0}\end{array}$ \\
\hline & & & & & & \\
\hline 1 & & & & 9526 & & 950 \\
\hline 2 & & & 50 & 862.81885 & 880 & 050 \\
\hline
\end{tabular}

7-Fluoro-2-(2-methylprop-1-en-1-yl)-3,4-dihydronaphthalen-1(2H)-one ((-)-13)

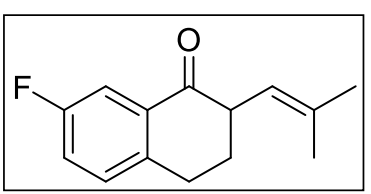

Prepared according to General procedure D:

1. Reaction: (32.7 mg, $0.15 \mathrm{mmol}, 75 \%)$, e.r. $82: 18$

2. Reaction: (31.0 mg, $0.14 \mathrm{mmol}, 71 \%)$, e.r. $82: 18$

$[\alpha]_{\mathrm{D}}^{25^{\circ} \mathrm{C}}=-61.4\left(\mathrm{c}=1.0\right.$ in $\mathrm{CHCl}_{3}$, e.r. $\left.82: 18\right)$

HPLC (AS-H column, eluent: $n$-hexane $/ i$-PrOH 99.8:0.2, flow rate: $0.5 \mathrm{~mL} / \mathrm{min}$ ): $\mathrm{t}_{\mathrm{R}}$ (major) $=$ $12.8 \mathrm{~min} ; \mathrm{t}_{\mathrm{R}}($ minor $)=15.2 \mathrm{~min}$. 
racemic sample:

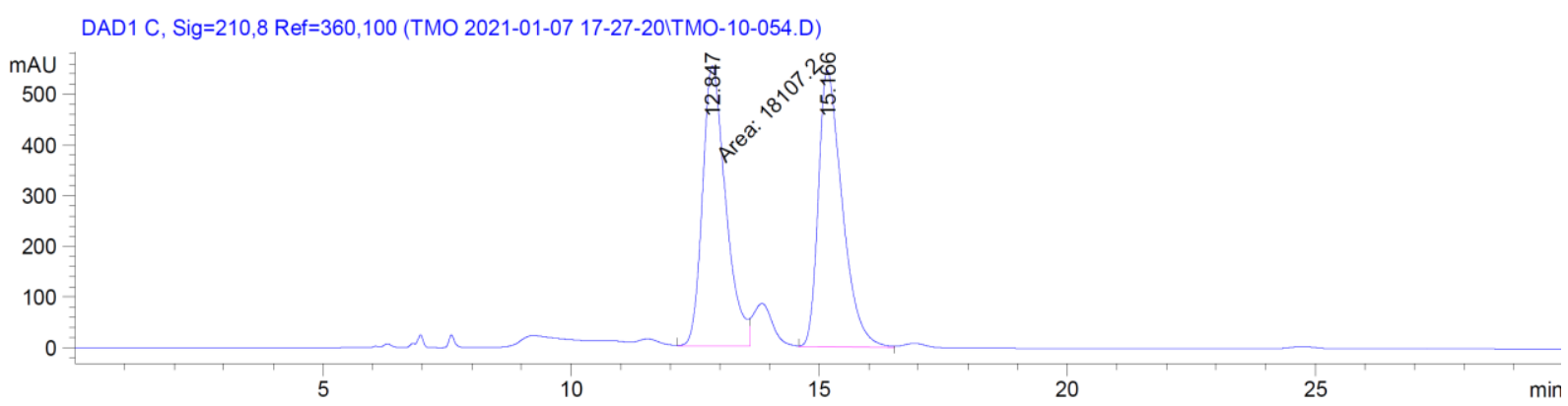

Signal 2: DAD1 C, Sig=210,8 Ref $=360,100$

\begin{tabular}{|c|c|c|c|c|c|c|}
\hline $\begin{array}{c}\text { Peak } \\
\#\end{array}$ & $\begin{array}{c}\text { RetTime } \\
\text { [min] }\end{array}$ & Type & $\begin{array}{l}\text { Width } \\
\text { [min] }\end{array}$ & $\begin{array}{c}\text { Area } \\
{\left[\mathrm{mAU}^{\star} \mathrm{s}\right]}\end{array}$ & $\begin{array}{l}\text { Height } \\
\text { [mAU] }\end{array}$ & $\begin{array}{c}\text { Area } \\
\frac{\circ}{0}\end{array}$ \\
\hline & & & & & & \\
\hline 1 & & IM & & 81 & 552 & 49 \\
\hline 0 & 66 & JB & 6 & $1.82877 \mathrm{e} 4$ & 8510 & 81 \\
\hline
\end{tabular}

enantioselective sample:

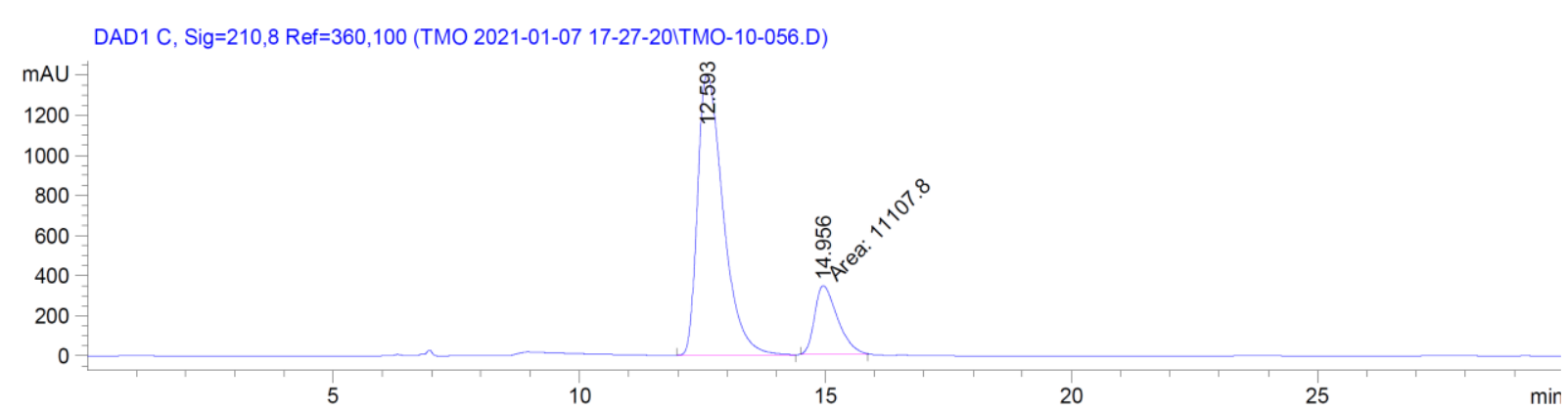

Signal 2: DAD1 C, Sig=210,8 Ref=360,100

\begin{tabular}{|c|c|c|c|c|c|c|}
\hline $\begin{array}{c}\text { Peak } \\
\text { \# }\end{array}$ & $\begin{array}{c}\text { RetTime } \\
\text { [min] }\end{array}$ & Type & $\begin{array}{l}\text { Width } \\
\text { [min] }\end{array}$ & $\begin{array}{c}\text { Area } \\
{\left[\mathrm{mAU}^{\star} \mathrm{s}\right]}\end{array}$ & $\begin{array}{l}\text { Height } \\
\text { [mAU] }\end{array}$ & $\begin{array}{c}\text { Area } \\
\frac{\circ}{0}\end{array}$ \\
\hline & & & & & & 82 \\
\hline 2 & 14.956 & & .5403 & $1.11078 \mathrm{e} 4$ & 342.61609 & 17.8986 \\
\hline
\end{tabular}

7-Bromo-2-(2-methylprop-1-en-1-yl)-3,4-dihydronaphthalen-1(2H)-one ((-)-14)

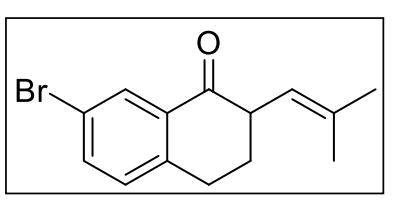

Prepared according to General procedure D:

1. Reaction: (30.7 mg, $0.11 \mathrm{mmol}, 55 \%)$, e.r. $69: 31$

2. Reaction: (28.7 mg, $0.10 \mathrm{mmol}, 51 \%)$, e.r. $69: 31$

$[\alpha]_{\mathrm{D}}^{25^{\circ} \mathrm{C}}=-33.6\left(\mathrm{c}=1.0\right.$ in $\mathrm{CHCl}_{3}$, e.r. $\left.69: 31\right)$

HPLC (AS-H column, eluent: $n$-hexane $/ i$-PrOH 99.8:0.2, flow rate: $0.5 \mathrm{~mL} / \mathrm{min}$ ): $\mathrm{t}_{\mathrm{R}}$ (major) $=$ $14.1 \mathrm{~min} ; \mathrm{t}_{\mathrm{R}}($ minor $)=15.9 \mathrm{~min}$. 
racemic sample:

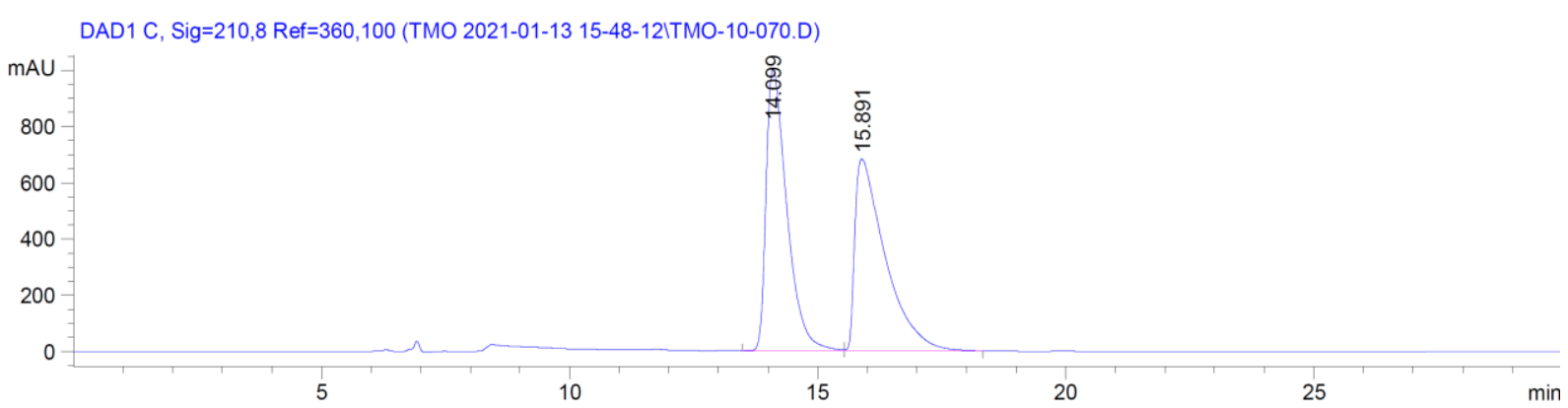

Signal 2: DAD1 C, Sig=210,8 Ref $=360,100$

\begin{tabular}{|c|c|c|c|c|c|c|}
\hline $\begin{array}{c}\text { eak } \\
\#\end{array}$ & $\begin{array}{c}\text { RetTime } \\
\text { [min] }\end{array}$ & Type & $\begin{array}{l}\text { Width } \\
{[\mathrm{min}]}\end{array}$ & $\begin{array}{c}\text { Area } \\
{\left[\mathrm{mAU}^{\star} \mathrm{s}\right]}\end{array}$ & $\begin{array}{l}\text { Height } \\
\text { [mAU] }\end{array}$ & $\begin{array}{c}\text { Area } \\
\quad \%\end{array}$ \\
\hline & & & & & & \\
\hline 1 & & & & & & 45 \\
\hline 2 & 5.8 & & & $2.91835 e 4$ & 682.63403 & 49 \\
\hline
\end{tabular}

enantioselective sample:

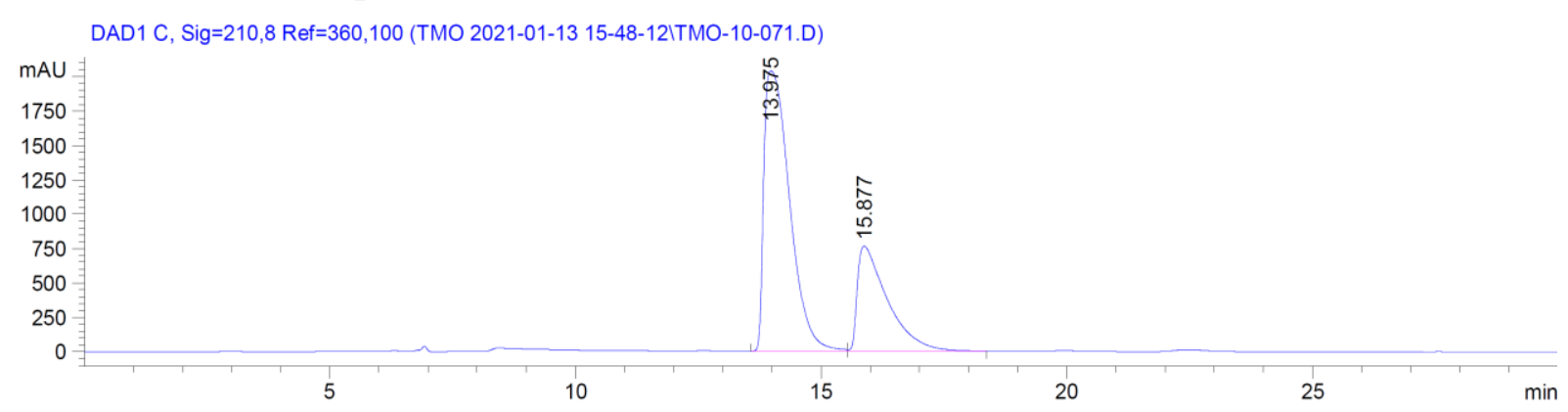

Signal 2: DAD1 C, Sig=210,8 $\operatorname{Ref}=360,100$

\begin{tabular}{|c|c|c|c|c|c|c|}
\hline $\begin{array}{c}\text { Peak } \\
\#\end{array}$ & $\begin{array}{c}\text { RetTime } \\
\text { [min] }\end{array}$ & Type & $\begin{array}{c}\text { Width } \\
\text { [min] }\end{array}$ & $\begin{array}{c}\text { Area } \\
{\left[\mathrm{mAU}{ }^{\star} \mathrm{s}\right]}\end{array}$ & $\begin{array}{l}\text { Height } \\
\text { [mAU] }\end{array}$ & $\begin{array}{c}\text { Area } \\
\frac{\circ}{0}\end{array}$ \\
\hline--- & ------- & & -- & --------- & ---1 & ----1 \\
\hline 1 & 13.975 & BV & 5716 & 7.41141e4 & 2041.37183 & 69.3839 \\
\hline 2 & 15.877 & VB & 0.6222 & $3.27034 \mathrm{e} 4$ & 765.76990 & 30.6161 \\
\hline
\end{tabular}

5,7-Dimethyl-2-(2-methylprop-1-en-1-yl)-3,4-dihydronaphthalen-1(2H)-one ((-)-15)

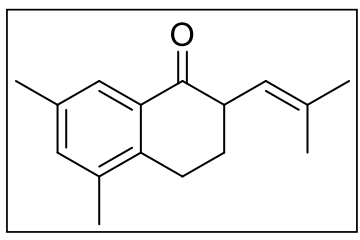

Prepared according to General procedure D:

1. Reaction: (39.7 mg, $0.17 \mathrm{mmol}, 87 \%)$, e.r. $93: 7$

2. Reaction: (38.6 mg, $0.17 \mathrm{mmol}, 84 \%)$, e.r. $93: 7$

$[\alpha]_{\mathrm{D}}^{25^{\circ} \mathrm{C}}=-56.7\left(\mathrm{c}=1.0\right.$ in $\mathrm{CHCl}_{3}$, e.r. $\left.93: 7\right)$ 
The enantiomer (+)-15 can be obtained using quinidine as catalyst:

1. Reaction: (38.0 mg, $0.17 \mathrm{mmol}, 83 \%)$, e.r. 7:93

2. Reaction: (39.9 mg, $0.17 \mathrm{mmol}, 87 \%$ ), e.r. 7:93

HPLC (AS-H column, eluent: $n$-hexane $/ i$-PrOH 99.8:0.2, flow rate: $0.5 \mathrm{~mL} / \mathrm{min}$ ): $\mathrm{t}_{\mathrm{R}}$ (major) $=$ $13.4 \mathrm{~min} ; \mathrm{t}_{\mathrm{R}}(\operatorname{minor})=17.6 \mathrm{~min}$.

racemic sample:

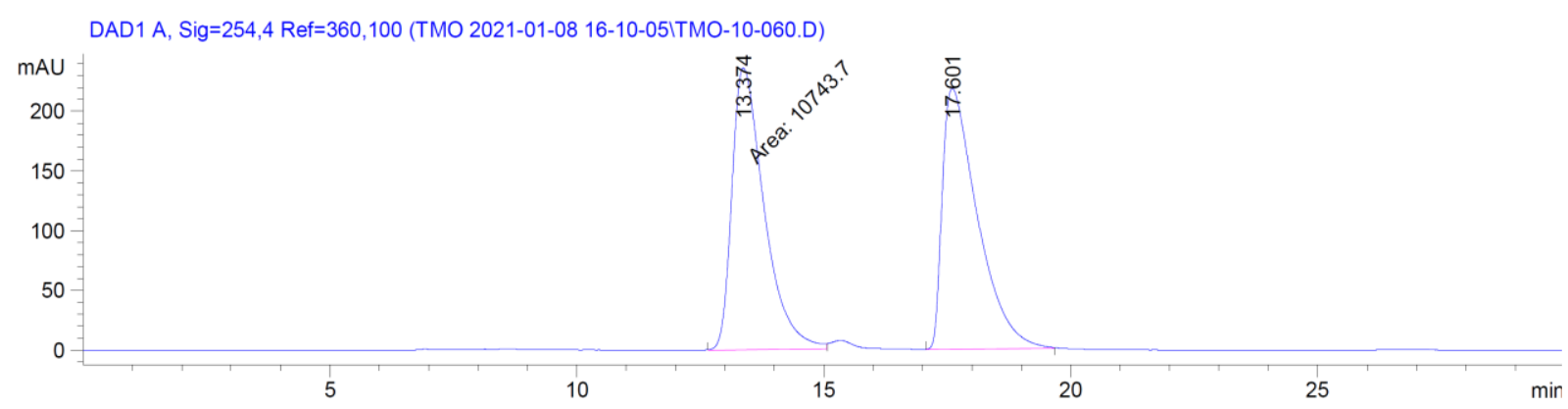

Signal 1: DAD1 A, Sig=254,4 Ref=360,100

\begin{tabular}{|c|c|c|c|c|c|c|}
\hline $\begin{array}{c}\text { Peak } \\
\text { \# }\end{array}$ & $\begin{array}{c}\text { RetTime } \\
\text { [min] }\end{array}$ & Type & $\begin{array}{l}\text { Width } \\
\text { [min] }\end{array}$ & $\begin{array}{c}\text { Area } \\
{\left[\mathrm{mAU}^{*} \mathrm{~s}\right]}\end{array}$ & $\begin{array}{l}\text { Height } \\
{[\mathrm{mAU}]}\end{array}$ & $\begin{array}{c}\text { Area } \\
\frac{\circ}{0}\end{array}$ \\
\hline & & & 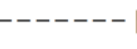 & --- & ---------- & $-------\mid$ \\
\hline 1 & 3.374 & & .7583 & $1.07437 \mathrm{e} 4$ & 236.13434 & 49.8607 \\
\hline 2 & 17.601 & & .7417 & $1.08037 \mathrm{e} 4$ & 218.19437 & 50.1393 \\
\hline
\end{tabular}

enantioselective sample: (-)-15

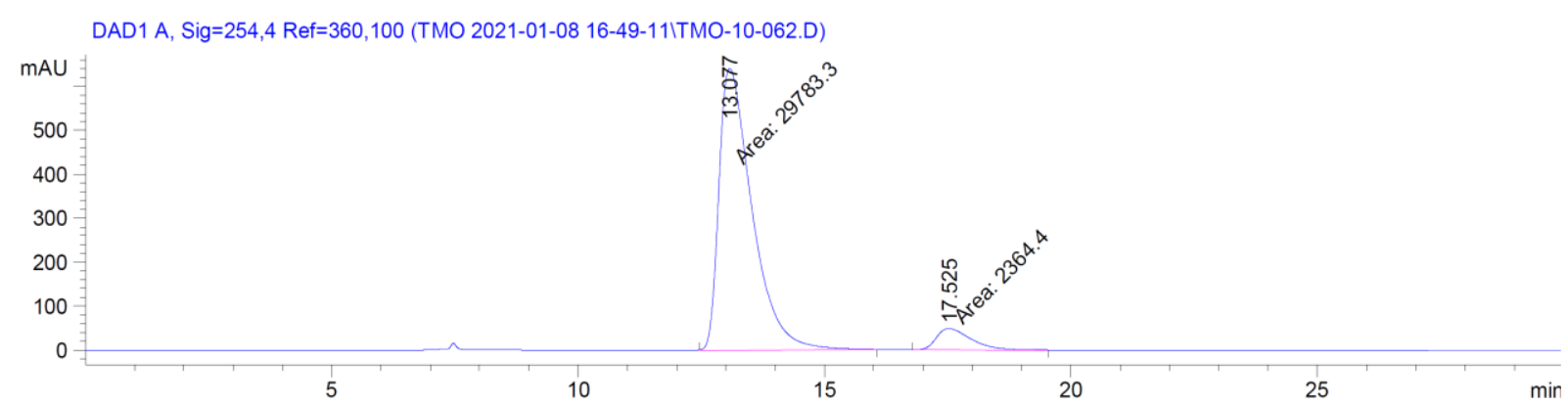

Signal 1: DAD1 A, Sig=254,4 Ref=360,100

\begin{tabular}{|c|c|c|c|c|c|c|}
\hline $\begin{array}{c}\text { Peak } \\
\#\end{array}$ & $\begin{array}{c}\text { RetTime } \\
\text { [min] }\end{array}$ & Type & $\begin{array}{l}\text { Width } \\
\text { [min] }\end{array}$ & $\begin{array}{c}\text { Area } \\
{\left[\mathrm{mAU}^{*} \mathrm{~s}\right]}\end{array}$ & $\begin{array}{l}\text { Height } \\
\text { [mAU] }\end{array}$ & $\begin{array}{c}\text { Area } \\
\frac{\circ}{0}\end{array}$ \\
\hline & & & & $\mid----$ & ---- & $--------\mid$ \\
\hline 1 & 13.077 & MM & & $2.97833 e 4$ & 639.35852 & 6452 \\
\hline 2 & 17.525 & MM & 0.8107 & 2364.39819 & 48.60907 & .3548 \\
\hline
\end{tabular}


enantioselective sample: $(+)-15$

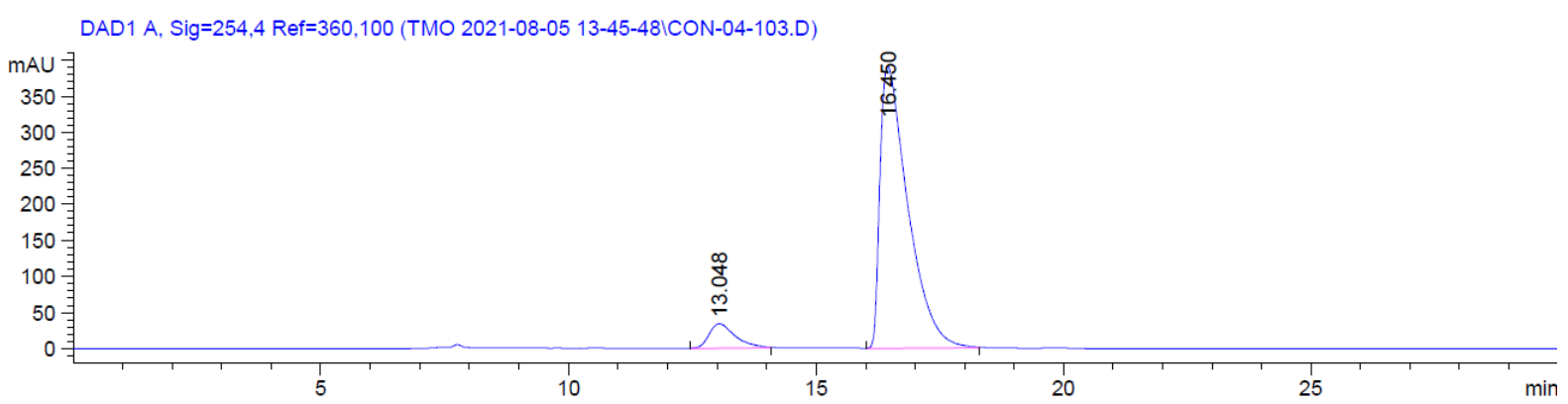

Signal 1: DAD1 A, Sig=254,4 Ref=360,100

\begin{tabular}{|c|c|c|c|c|c|c|}
\hline $\begin{array}{c}\text { Peak } \\
\#\end{array}$ & $\begin{array}{c}\text { RetTime } \\
\text { [min] }\end{array}$ & Type & $\begin{array}{l}\text { Width } \\
\text { [min] }\end{array}$ & $\begin{array}{c}\text { Area } \\
{\left[\mathrm{mAU}^{*} \mathrm{~s}\right]}\end{array}$ & $\begin{array}{l}\text { Height } \\
{[\mathrm{mAU}]}\end{array}$ & $\begin{array}{c}\text { Area } \\
\%\end{array}$ \\
\hline & 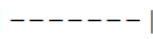 & & 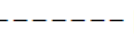 & --------- & -- & - \\
\hline 1 & 13.048 & $\mathrm{BB}$ & 0.5482 & 1223.65601 & 33.65299 & 7.3952 \\
\hline 2 & 16.450 & $3 \mathrm{R}$ & 0.5915 & $1.53230 \mathrm{e} 4$ & 390.73984 & 92.6048 \\
\hline
\end{tabular}

\section{3-(2-Methylprop-1-en-1-yl)chroman-4-one ((-)-16)}

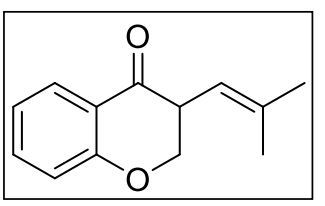

Prepared according to General procedure D:

1. Reaction: (39.8 mg, $0.20 \mathrm{mmol}, 98 \%)$, e.r. $81: 19$

2. Reaction: (37.1 mg, $0.18 \mathrm{mmol}, 92 \%)$, e.r. $80: 20$

$$
[\alpha]_{\mathrm{D}}^{25^{\circ} \mathrm{C}}=-75.0\left(\mathrm{c}=1.0 \text { in } \mathrm{CHCl}_{3} \text {, e.r. } 80: 20\right)
$$

HPLC (AS-H column, eluent: $n$-hexane $/ i$-PrOH 99:1, flow rate: $0.5 \mathrm{~mL} / \mathrm{min}$ ): $\mathrm{t}_{\mathrm{R}}$ (major) $=$ $12.9 \mathrm{~min} ; \mathrm{t}_{\mathrm{R}}(\operatorname{minor})=15.0 \mathrm{~min}$.

racemic sample:

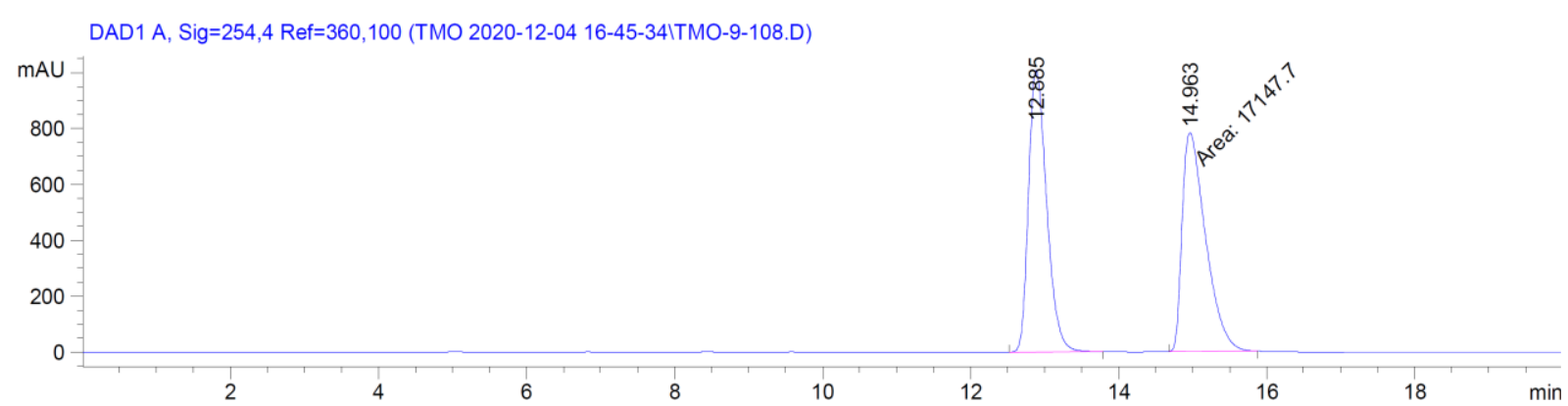

Signal 1: DAD1 A, Sig=254,4 Ref=360,100

\begin{tabular}{|c|c|c|c|c|c|c|}
\hline $\begin{array}{c}\text { eak } \\
\#\end{array}$ & $\begin{array}{c}\text { RetTime } \\
\text { [min] }\end{array}$ & Type & $\begin{array}{c}\text { Width } \\
\text { [min] }\end{array}$ & $\begin{array}{c}\text { Area } \\
{\left[\mathrm{mAU}^{\star} \mathrm{s}\right]}\end{array}$ & $\begin{array}{l}\text { Height } \\
\text { [mAU] }\end{array}$ & $\begin{array}{c}\text { Area } \\
\quad \circ\end{array}$ \\
\hline & & & & & $\mid---$ & \\
\hline & & & & $5 e 4$ & .85663 & 580 \\
\hline 2 & 4.963 & & 655 & $1.71477 \mathrm{e} 4$ & 781.82953 & 49.9420 \\
\hline
\end{tabular}


enantioselective sample:

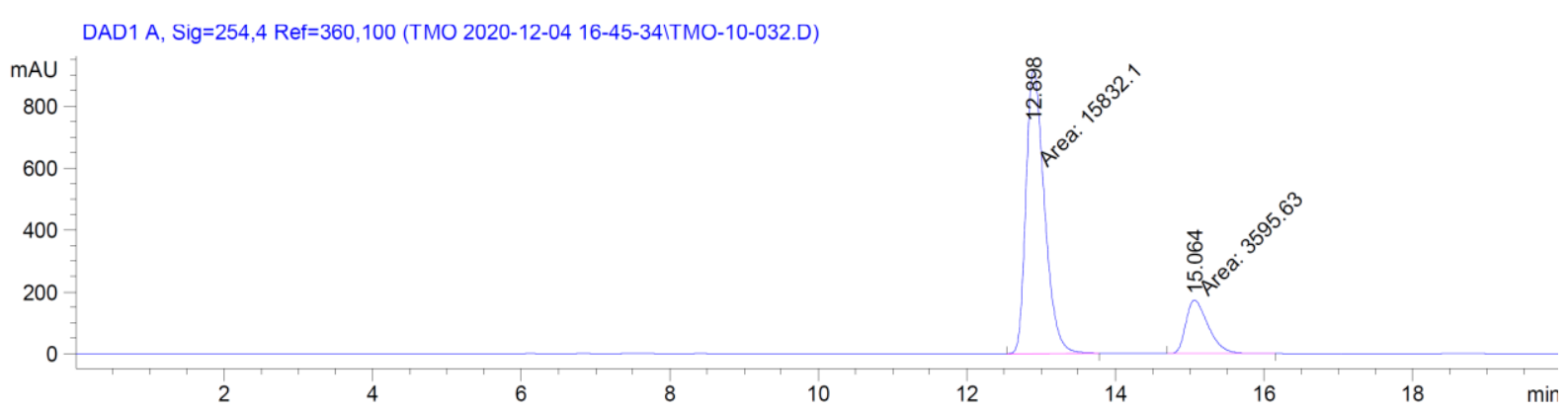

Signal 1: DAD1 A, Sig=254,4 Ref=360,100

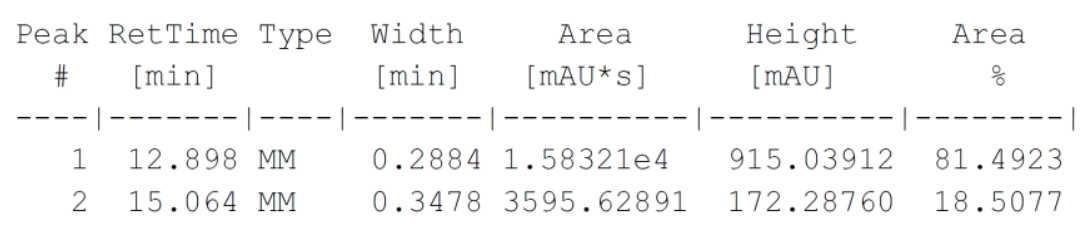

6-(2-Methylprop-1-en-1-yl)-6,7,8,9-tetrahydro-5H-benzo[7]annulen-5-one ((+)-18)

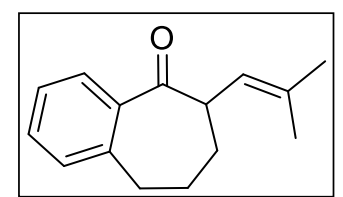

Prepared according to General procedure D:

1. Reaction: (39.3 mg, $0.18 \mathrm{mmol}, 92 \%)$, e.r. $95: 5$

2. Reaction: (38.1 mg, $0.18 \mathrm{mmol}, 89 \%)$, e.r. $95: 5$

$[\alpha]_{\mathrm{D}}^{25^{\circ} \mathrm{C}}=+41.9\left(\mathrm{c}=1.0\right.$ in $\mathrm{CHCl}_{3}$, e.r. $\left.95: 5\right)$

The enantiomer (-)-18 can be obtained using quinidine as catalyst:

1. Reaction: (42.4 mg, $0.20 \mathrm{mmol}, 99 \%)$, e.r. 7:93

2. Reaction: (42.8 mg, $0.20 \mathrm{mmol}$, quant.), e.r. 7:93

HPLC (AS-H column, eluent: $n$-hexane $/ i$-PrOH 99.8:0.2, flow rate: $0.5 \mathrm{~mL} / \mathrm{min}): \mathrm{t}_{\mathrm{R}}($ major $)=$ $12.0 \min ; \mathrm{t}_{\mathrm{R}}(\operatorname{minor})=13.9 \mathrm{~min}$.

racemic sample: 
DAD1 A, Sig=254,4 Ref=360, 100 (TMO 2020-12-07 13-56-19ITMO-10-110.D)

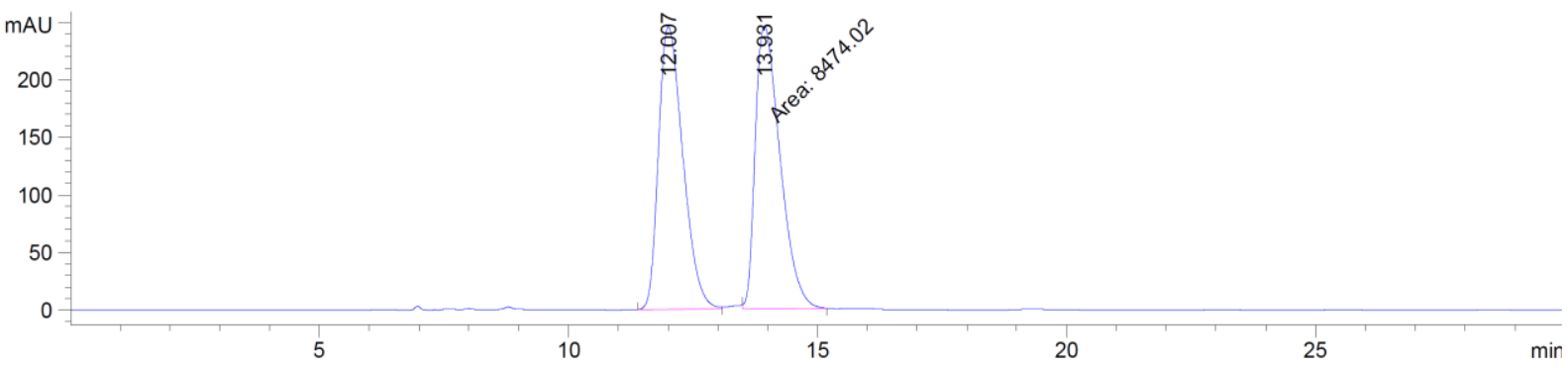

Signal 1: DAD1 A, Sig=254, 4 Ref=360,100

\begin{tabular}{|c|c|c|c|c|c|c|}
\hline $\begin{array}{c}\text { eak } \\
\#\end{array}$ & $\begin{array}{c}\text { RetTime } \\
\text { [min] }\end{array}$ & Type & $\begin{array}{l}\text { Width } \\
\text { [min] }\end{array}$ & $\begin{array}{c}\text { Area } \\
{\left[\mathrm{mAU}{ }^{*} \mathrm{~s}\right]}\end{array}$ & $\begin{array}{l}\text { Height } \\
{[\mathrm{mAU}]}\end{array}$ & $\begin{array}{c}\text { Area } \\
\quad \%\end{array}$ \\
\hline & & & & | ----------- & --- & --- \\
\hline 7 & דתחק & $\mathrm{BB}$ & 349 & 8399.67676 & 245.67989 & 797 \\
\hline 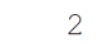 & 3.931 & MM & 749 & 8474.02051 & 245.67204 & 203 \\
\hline
\end{tabular}

enantioselective sample: (+)-18

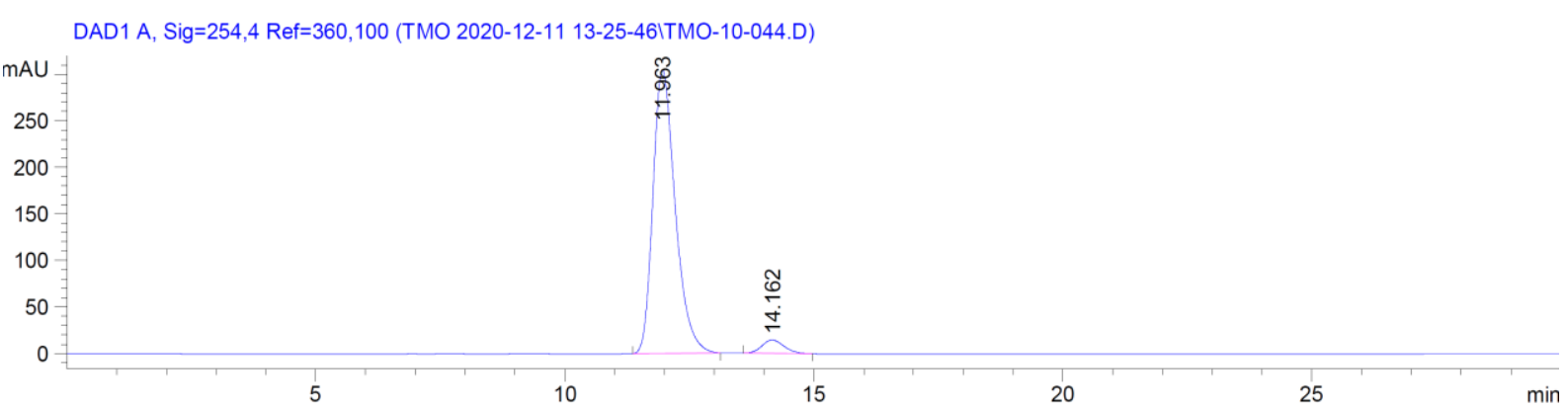

Signal 1: DAD1 A, Sig=254,4 Ref=360,100

\begin{tabular}{|c|c|c|c|c|c|c|}
\hline $\begin{array}{c}\text { Peak } \\
\#\end{array}$ & $\begin{array}{c}\text { RetTime } \\
\text { [min] }\end{array}$ & Type & $\begin{array}{l}\text { Width } \\
\text { [min] }\end{array}$ & $\begin{array}{c}\text { Area } \\
{\left[\mathrm{mAU}^{*} \mathrm{~s}\right]}\end{array}$ & $\begin{array}{l}\text { Height } \\
{[\mathrm{mAU}]}\end{array}$ & $\begin{array}{c}\text { Area } \\
\frac{\circ}{\circ}\end{array}$ \\
\hline & $\mid-------$ & 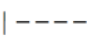 & & ----------- & $----------\mid$ & $--------\mid$ \\
\hline 1 & 11.963 & $\mathrm{BB}$ & 0.4791 & 9541.51758 & 304.90732 & 95.4252 \\
\hline 2 & 14.162 & $\mathrm{BB}$ & 0.4863 & 457.43231 & 14.56915 & 4.5748 \\
\hline
\end{tabular}

enantioselective sample: (-)-18

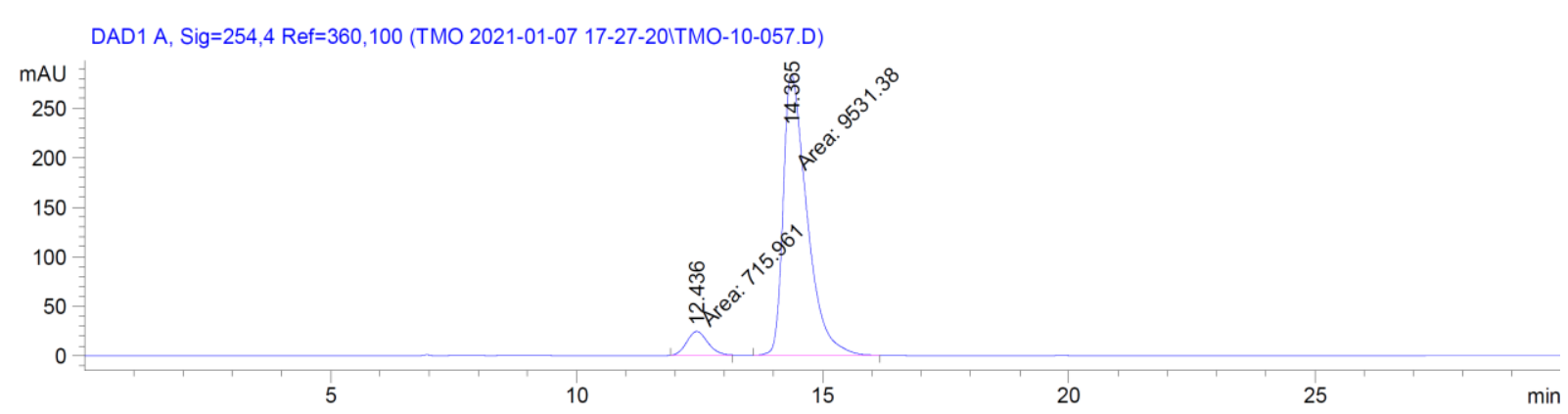


Signal 1: DAD1 A, Sig=254,4 Ref $=360,100$

\begin{tabular}{|c|c|c|c|c|c|c|}
\hline $\begin{array}{c}\text { eak } \\
\#\end{array}$ & $\begin{array}{l}\text { RetTime } \\
\text { [min] }\end{array}$ & Type & $\begin{array}{l}\text { Width } \\
\text { [min] }\end{array}$ & $\begin{array}{c}\text { Area } \\
{\left[\mathrm{mAU}^{\star} \mathrm{s}\right]}\end{array}$ & $\begin{array}{l}\text { Height } \\
\text { [mAU] }\end{array}$ & $\begin{array}{c}\text { Area } \\
\frac{\circ}{0}\end{array}$ \\
\hline 1 & & & & & & 68 \\
\hline 2 & 14.365 & t. & 03 & 9531.37891 & 283.53250 & 132 \\
\hline
\end{tabular}

6-(2-Ethylbut-1-en-1-yl)-6,7,8,9-tetrahydro-5H-benzo[7]annulen-5-one ((+)-19)

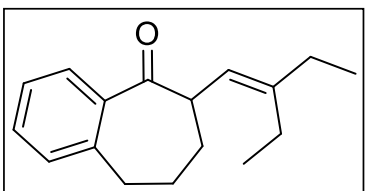

Prepared according to General procedure D:

1. Reaction: (45.2 mg, $0.19 \mathrm{mmol}, 93 \%)$, e.r. $92: 8$

2. Reaction: (42.5 mg, $0.18 \mathrm{mmol}, 88 \%)$, e.r. $93: 7$

$[\alpha]_{\mathrm{D}}^{25^{\circ} \mathrm{C}}=+16.2\left(\mathrm{c}=1.0\right.$ in $\mathrm{CHCl}_{3}$, e.r. $\left.93: 7\right)$

The enantiomer (-)-19 can be obtained using quinidine as catalyst:

1. Reaction: (41.8 mg, $0.17 \mathrm{mmol}, 86 \%)$, e.r. $6: 94$

2. Reaction: (40.8 mg, $0.17 \mathrm{mmol}, 84 \%)$, e.r. $7: 93$

HPLC (AS-H column, eluent: $n$-hexane $/ i$-PrOH 99.9:0.1, flow rate: $0.5 \mathrm{~mL} / \mathrm{min}): \mathrm{t}_{\mathrm{R}}($ major $)=$ $25.0 \mathrm{~min} ; \mathrm{t}_{\mathrm{R}}($ minor $)=30.2 \mathrm{~min}$.

racemic sample:

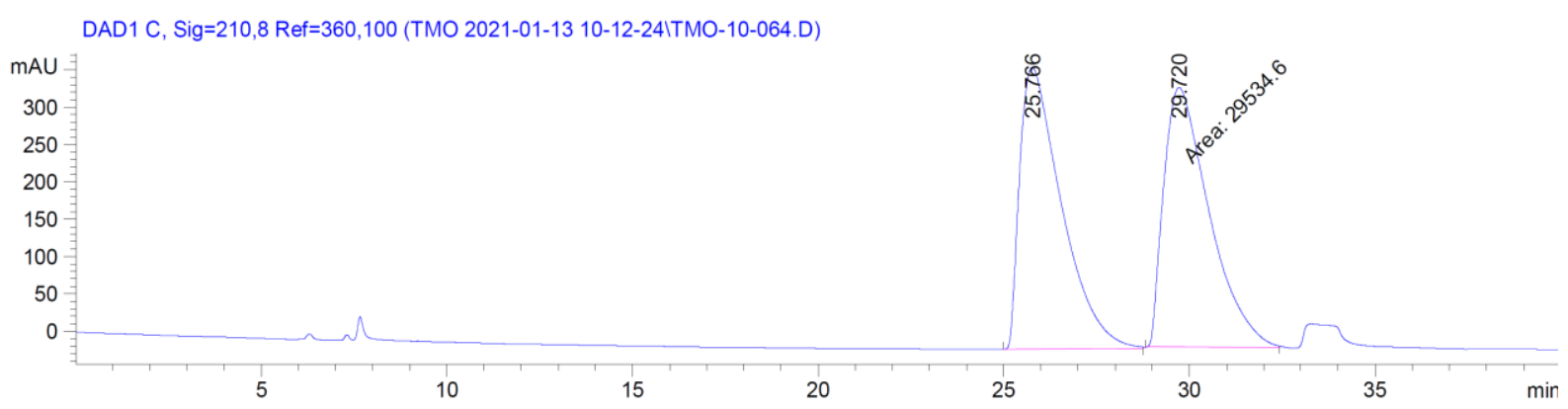

Signal 1: DAD1 A, Sig=254,4 Ref=360,100

\begin{tabular}{|c|c|c|c|c|c|c|}
\hline $\begin{array}{c}\text { Peak } \\
\quad \#\end{array}$ & $\begin{array}{c}\text { RetTime } \\
\text { [min] }\end{array}$ & Type & $\begin{array}{l}\text { Width } \\
\text { [min] }\end{array}$ & $\begin{array}{c}\text { Area } \\
{\left[\mathrm{mAU}^{\star} \mathrm{s}\right]}\end{array}$ & $\begin{array}{l}\text { Height } \\
\text { [mAU] }\end{array}$ & $\begin{array}{c}\text { Area } \\
\frac{\circ}{0}\end{array}$ \\
\hline & & & & $-----\mid$ & $---------\mid$ & $--------\mid$ \\
\hline 1 & & $4 \mathrm{M}$ & 09 & 4405.56152 & 56.44123 & 50.4995 \\
\hline 2 & 29.720 & MM & 47 & 4318.40332 & 51.60574 & 49.5005 \\
\hline
\end{tabular}


enantioselective sample: (+)-19

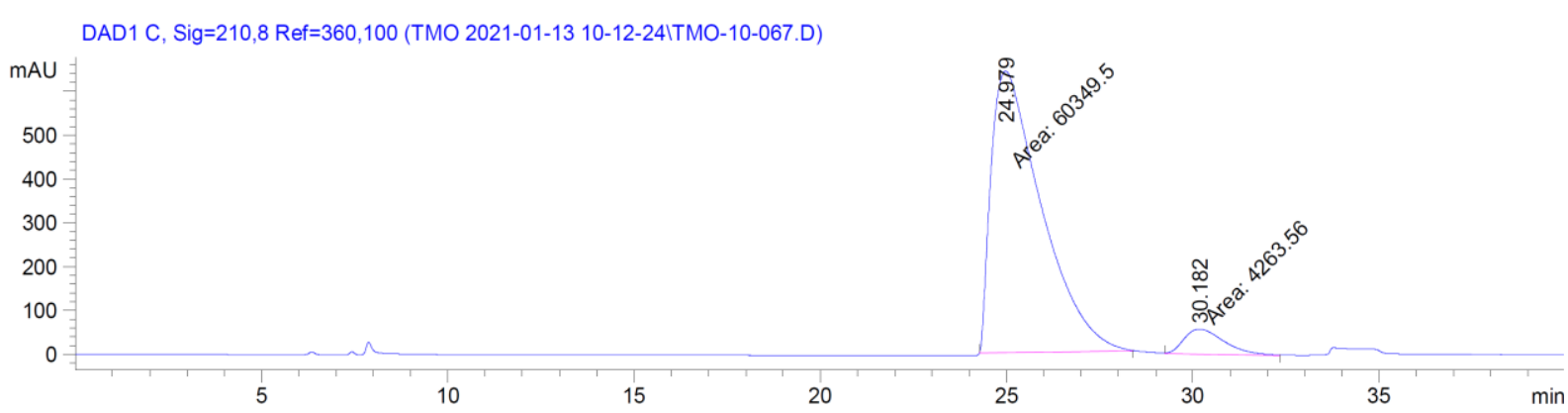

Signal 2: DAD1 C, Sig=210,8 Ref=360,100

\begin{tabular}{|c|c|c|c|c|c|c|}
\hline $\begin{array}{c}\text { Peak } \\
\#\end{array}$ & $\begin{array}{c}\text { RetTime } \\
\text { [min] }\end{array}$ & Type & $\begin{array}{l}\text { Width } \\
\text { [min] }\end{array}$ & $\begin{array}{c}\text { Area } \\
{\left[\mathrm{mAU}{ }^{*} \mathrm{~S}\right]}\end{array}$ & $\begin{array}{l}\text { Height } \\
{[\mathrm{mAU}]}\end{array}$ & $\begin{array}{c}\text { Area } \\
\quad \frac{\circ}{0}\end{array}$ \\
\hline & & & 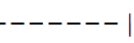 & -------- & --- & 1 \\
\hline 1 & 24.979 & MM & 1.5660 & $6.03495 e 4$ & 642.27386 & 93.4014 \\
\hline 2 & 30.182 & $\mathrm{MM}$ & 1.2423 & 4263.56396 & 0154 & 986 \\
\hline
\end{tabular}

enantioselective sample: $(-)-19$

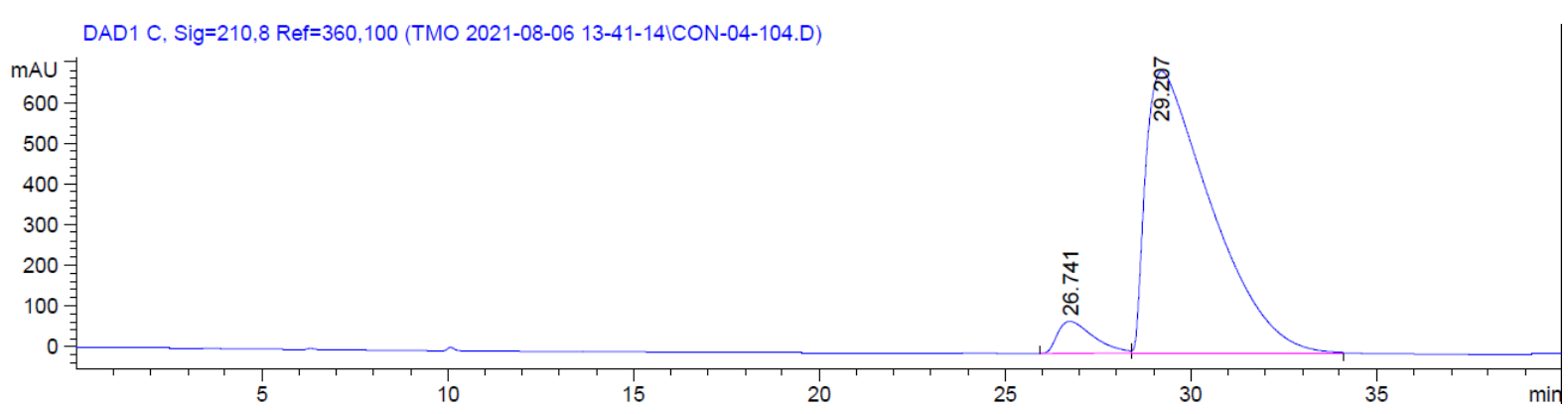

Signal 2: DAD1 C, Sig=210,8 Ref=360,100

\begin{tabular}{|c|c|c|c|c|c|c|}
\hline $\begin{array}{c}\text { Peak } \\
\quad \#\end{array}$ & $\begin{array}{c}\text { RetTime } \\
\text { [min] }\end{array}$ & Type & $\begin{array}{l}\text { Width } \\
\text { [min] }\end{array}$ & $\begin{array}{c}\text { Area } \\
{\left[\mathrm{mAU}^{*} \mathrm{~s}\right]}\end{array}$ & $\begin{array}{l}\text { Height } \\
{[\mathrm{mAU}]}\end{array}$ & $\begin{array}{c}\text { Area } \\
\quad \%\end{array}$ \\
\hline & & & & ---- & ----- & . \\
\hline 1 & 26.741 & BV & .0084 & 5297.95508 & 78.59802 & 344 \\
\hline 0 & 29.207 & VB & 40 & $8.47358 e 4$ & 1869 & 56 \\
\hline
\end{tabular}

tert-Butyl 4-((5-oxo-6,7,8,9-tetrahydro-5H-benzo[7]annulen-6-yl)methylene)piperidine-1carboxylate $((+)-20)$

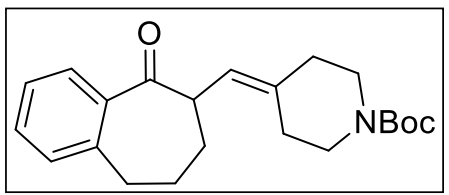

Prepared according to General procedure D:

1. Reaction: (64.0 mg, $0.18 \mathrm{mmol}, 90 \%)$, e.r. $90: 10$

2. Reaction: (62.6 mg, $0.18 \mathrm{mmol}, 88 \%)$, e.r. $90: 10$

$[\alpha]_{\mathrm{D}}^{25^{\circ} \mathrm{C}}=+7.7\left(\mathrm{c}=1.0\right.$ in $\mathrm{CHCl}_{3}$, e.r. $\left.90: 10\right)$ 
The enantiomer (-)-20 can be obtained using quinidine as catalyst:

1. Reaction: (69.1 mg, $0.19 \mathrm{mmol}, 97 \%)$, e.r. 8:92

2. Reaction: (72.0 mg, $0.20 \mathrm{mmol}$, quant.), e.r. 8:92

HPLC (AS-H column, eluent: $n$-hexane $/ i-\mathrm{PrOH}$ 90:10, flow rate: $0.5 \mathrm{~mL} / \mathrm{min}$ ): $\mathrm{t}_{\mathrm{R}}$ (major) $=$ $9.4 \mathrm{~min} ; \mathrm{t}_{\mathrm{R}}($ minor $)=10.5 \mathrm{~min}$.

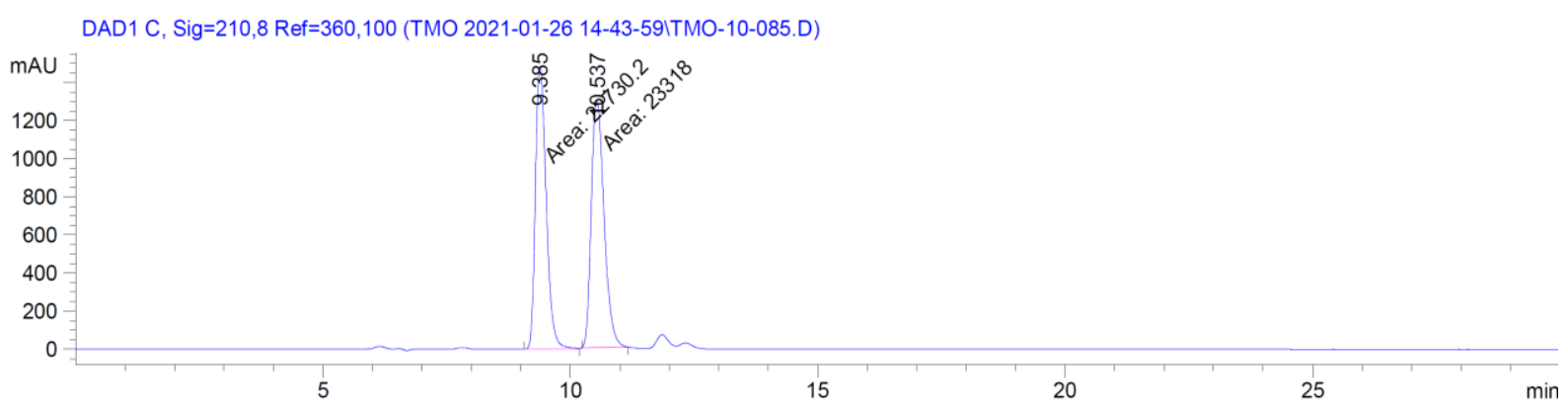

Signal 2: DAD1 C, Sig=210,8 Ref $=360,100$

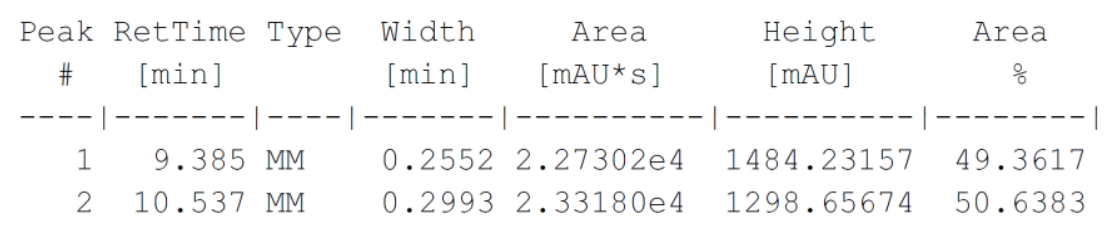

enantioselective sample: $(+)-20$

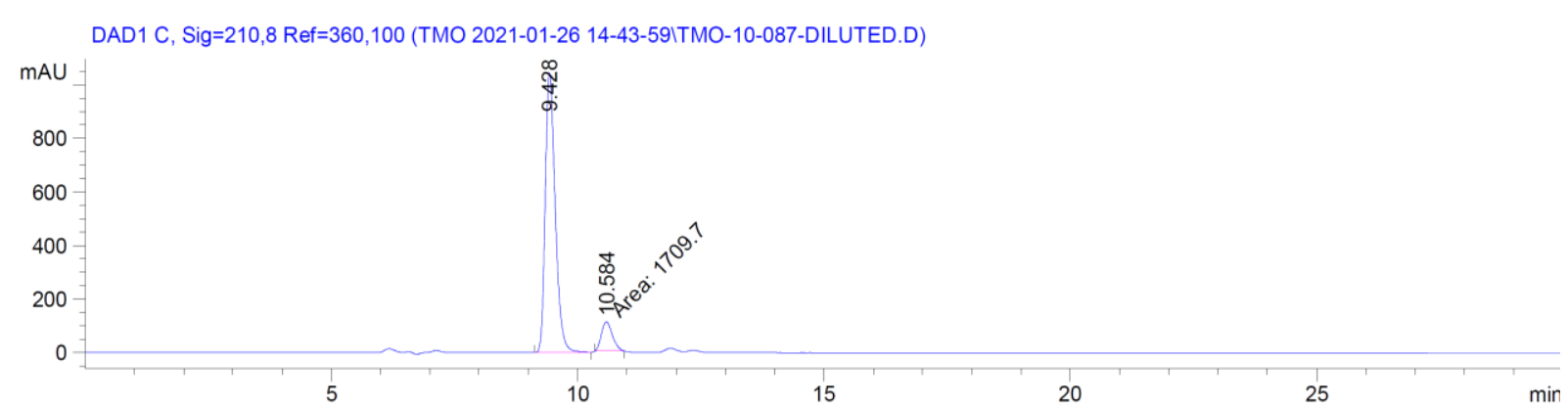

Signal 2: DAD1 C, Sig=210,8 Ref $=360,100$

\begin{tabular}{|c|c|c|c|c|c|c|}
\hline $\begin{array}{c}\text { eak } \\
\#\end{array}$ & $\begin{array}{c}\text { RetTime } \\
\text { [min] }\end{array}$ & Type & $\begin{array}{l}\text { Width } \\
\text { [min] }\end{array}$ & $\begin{array}{c}\text { Area } \\
{\left[\mathrm{mAU}{ }^{*} \mathrm{~s}\right]}\end{array}$ & $\begin{array}{l}\text { Height } \\
\text { [mAU] }\end{array}$ & $\begin{array}{c}\text { Area } \\
\frac{\circ}{0}\end{array}$ \\
\hline--1 & . & & & ---------- & $\mid----$ & $-------\mid$ \\
\hline 1 & 428 & BB & 4 & $1.48008 e 4$ & .87988 & 6448 \\
\hline 2 & 10.584 & MM & 583 & 1709.69897 & 110.33563 & 10.3552 \\
\hline
\end{tabular}


enantioselective sample: $(-)-20$

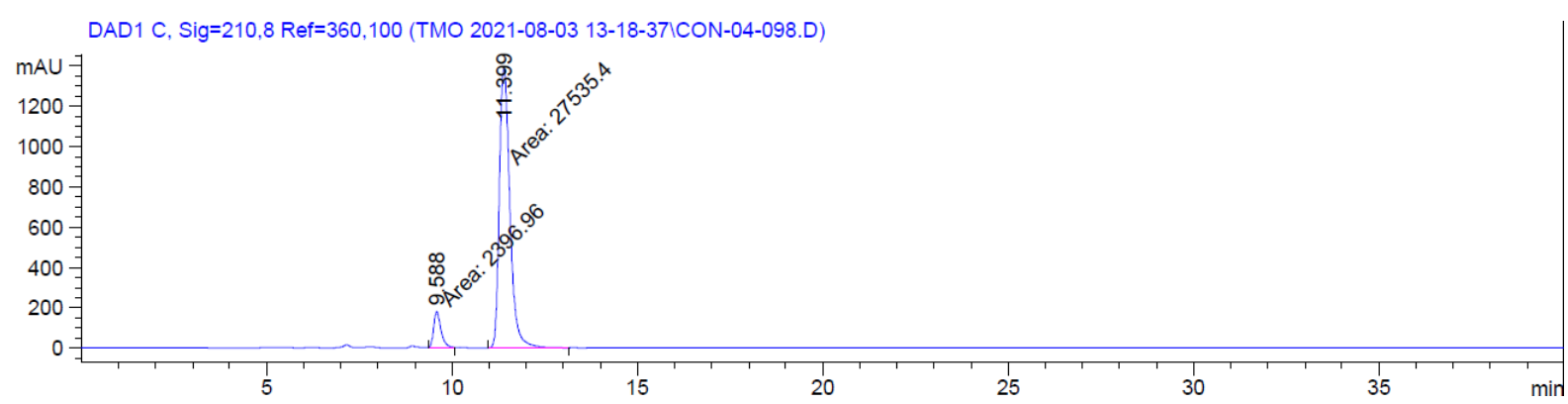

Signal 2: DAD1 C, Sig=210,8 Ref=360,100

\begin{tabular}{|c|c|c|c|c|c|c|}
\hline $\begin{array}{c}\text { Peak } \\
\quad \#\end{array}$ & $\begin{array}{c}\text { RetTime } \\
\text { [min] }\end{array}$ & Type & $\begin{array}{l}\text { Width } \\
\text { [min] }\end{array}$ & $\begin{array}{c}\text { Area } \\
{\left[\mathrm{mAU}^{\star} \mathrm{s}\right]}\end{array}$ & $\begin{array}{l}\text { Height } \\
{[\mathrm{mAU}]}\end{array}$ & $\begin{array}{c}\text { Area } \\
\frac{8}{8}\end{array}$ \\
\hline & ----- & ---1 & $-------\mid$ & $----------\mid$ & $----------\mid$ & $-------\mid$ \\
\hline 1 & 9.588 & $\mathrm{MM}$ & 0.2251 & 2396.96289 & 177.49065 & 8.0079 \\
\hline 2 & 11.399 & $\mathrm{MM}$ & 0.3306 & $2.75354 e 4$ & 1388.14368 & 91.9921 \\
\hline
\end{tabular}

6-((Tetrahydro-4H-pyran-4-ylidene)methyl)-6,7,8,9-tetrahydro-5H-benzo[7]annulen-5one $((+)-21)$

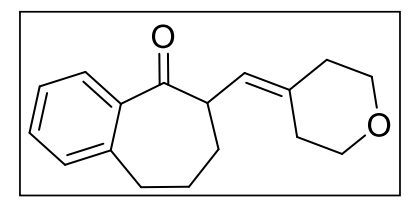

Prepared according to General procedure D:

1. Reaction: (47.2 mg, $0.18 \mathrm{mmol}, 92 \%)$, e.r. $91: 9$

2. Reaction: (44.8 mg, $0.17 \mathrm{mmol}, 87 \%)$, e.r. $92: 8$

$[\alpha]_{\mathrm{D}}^{25^{\circ} \mathrm{C}}=+17.4\left(\mathrm{c}=1.0\right.$ in $\mathrm{CHCl}_{3}$, e.r. $\left.91: 9\right)$

The enantiomer (-)-21 can be obtained using quinidine as catalyst:

1. Reaction: (49.6 mg, $0.19 \mathrm{mmol}, 97 \%)$, e.r. 10:90

2. Reaction: (49.0 mg, $0.19 \mathrm{mmol}, 96 \%)$, e.r. 8:92

HPLC (OJ-H column, eluent: $n$-hexane/i-PrOH 95:5, flow rate: $0.5 \mathrm{~mL} / \mathrm{min}): \mathrm{t}_{\mathrm{R}}$ (major) $=$ $22.7 \mathrm{~min} ; \mathrm{t}_{\mathrm{R}}($ minor $)=32.7 \mathrm{~min}$.

racemic sample:

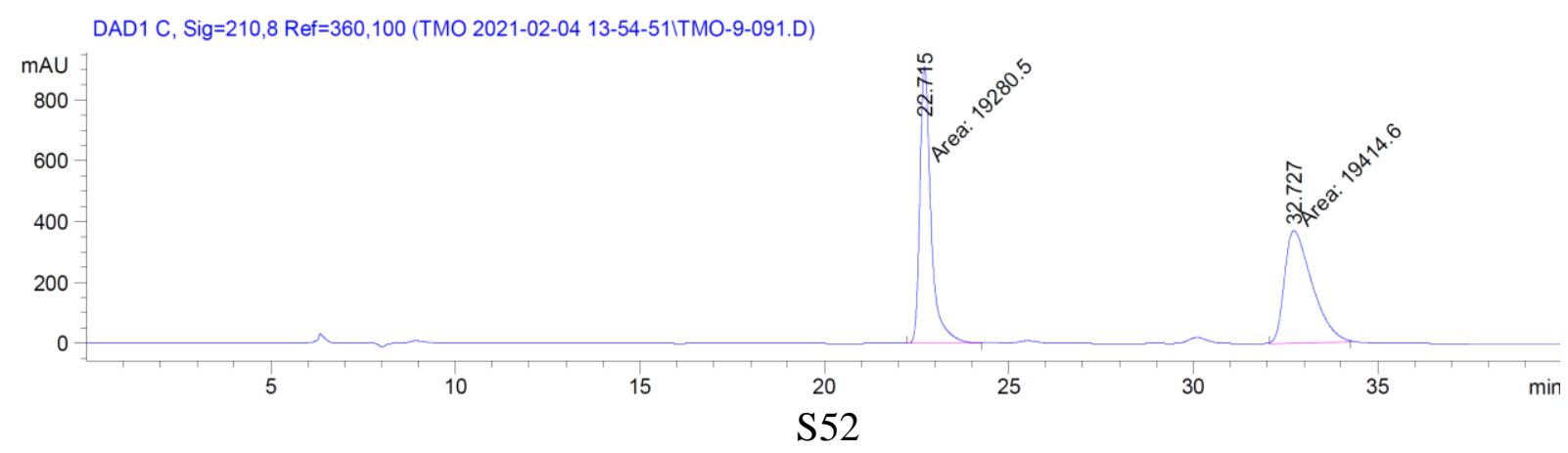


Signal 2: DAD1 C, Sig=210,8 Ref=360,100

\begin{tabular}{|c|c|c|c|c|c|c|}
\hline $\begin{array}{c}\text { eak } \\
\text { \# }\end{array}$ & $\begin{array}{c}\text { RetTime } \\
\text { [min] }\end{array}$ & Type & $\begin{array}{l}\text { Width } \\
\text { [min] }\end{array}$ & $\begin{array}{c}\text { Area } \\
{\left[\mathrm{mAU}{ }^{*} \mathrm{~s}\right]}\end{array}$ & $\begin{array}{l}\text { Height } \\
{[\mathrm{mAU}]}\end{array}$ & $\begin{array}{c}\text { Area } \\
\frac{\circ}{0}\end{array}$ \\
\hline & & & & & & \\
\hline 1 & & & & & & \\
\hline 2 & 32.727 & & 15 & $1.94146 \mathrm{e} 4$ & 371.30167 & 132 \\
\hline
\end{tabular}

enantioselective sample: (+)-21

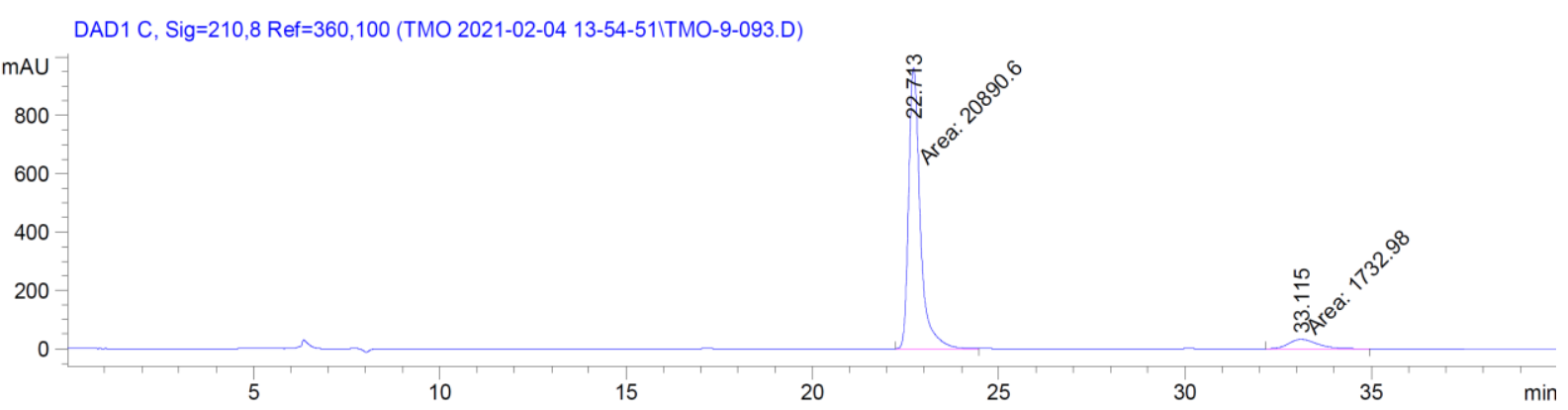

Signal 2: DAD1 C, Sig=210,8 $\operatorname{Ref}=360,100$

\begin{tabular}{|c|c|c|c|c|c|c|}
\hline $\begin{array}{c}\text { Peak } \\
\text { \# }\end{array}$ & $\begin{array}{c}\text { RetTime } \\
\text { [min] }\end{array}$ & Type & $\begin{array}{l}\text { Width } \\
\text { [min] }\end{array}$ & $\begin{array}{c}\text { Area } \\
{\left[\mathrm{mAU}^{*} \mathrm{~s}\right]}\end{array}$ & $\begin{array}{l}\text { Height } \\
\text { [mAU] }\end{array}$ & $\begin{array}{c}\text { Area } \\
\frac{\circ}{0}\end{array}$ \\
\hline & & & & --- & --- & \\
\hline 1 & 22 . & & & $2.08906 \mathrm{e} 4$ & 963.12280 & 399 \\
\hline 2 & 33.115 & & 847 & 1732.98169 & 32.64735 & 7.6601 \\
\hline
\end{tabular}

enantioselective sample: $(-)-21$

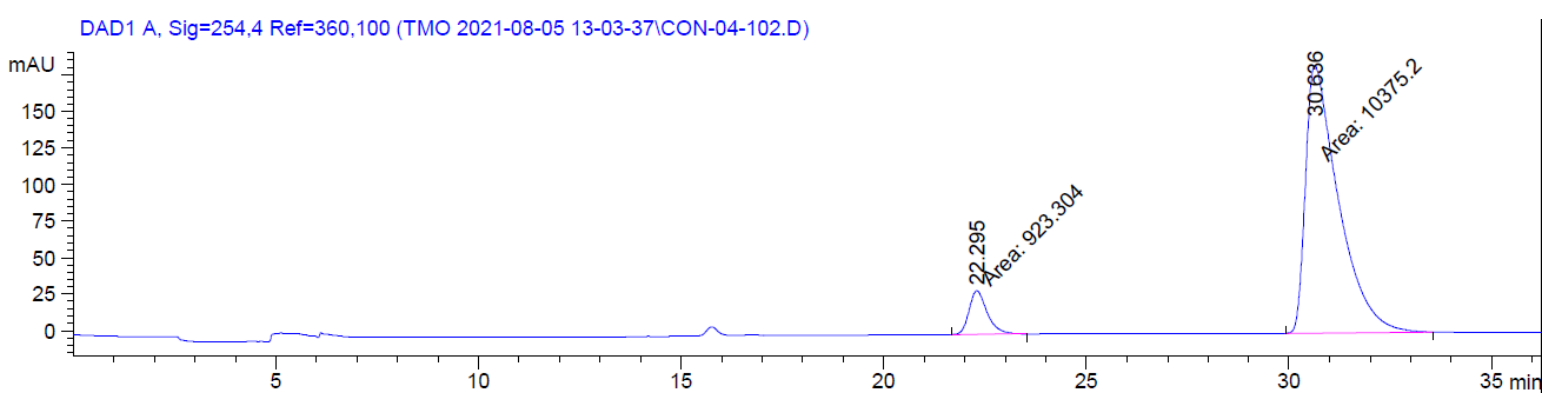

Signal 1: DAD1 A, Sig=254,4 Ref=360,100

\begin{tabular}{|c|c|c|c|c|c|c|}
\hline $\begin{array}{c}\text { Peak } \\
\#\end{array}$ & $\begin{array}{c}\text { RetTime } \\
\text { [min] }\end{array}$ & Type & $\begin{array}{l}\text { Width } \\
\text { [min] }\end{array}$ & $\begin{array}{c}\text { Area } \\
{\left[\mathrm{mAU}^{\star} \mathrm{S}\right]}\end{array}$ & $\begin{array}{l}\text { Height } \\
\text { [mAU] }\end{array}$ & $\begin{array}{c}\text { Area } \\
\frac{8}{8}\end{array}$ \\
\hline & & & & --- & -------- & -- \\
\hline 1 & 2. & $\mathbb{M}$ & 58 & 923.30383 & 29 & 19 \\
\hline 2 & 30.636 & M & 444 & $1.03752 \mathrm{e} 4$ & 183.09172 & 8281 \\
\hline
\end{tabular}




\section{Mechanistic Investigations}

\section{UV/vis Spectroscopy}

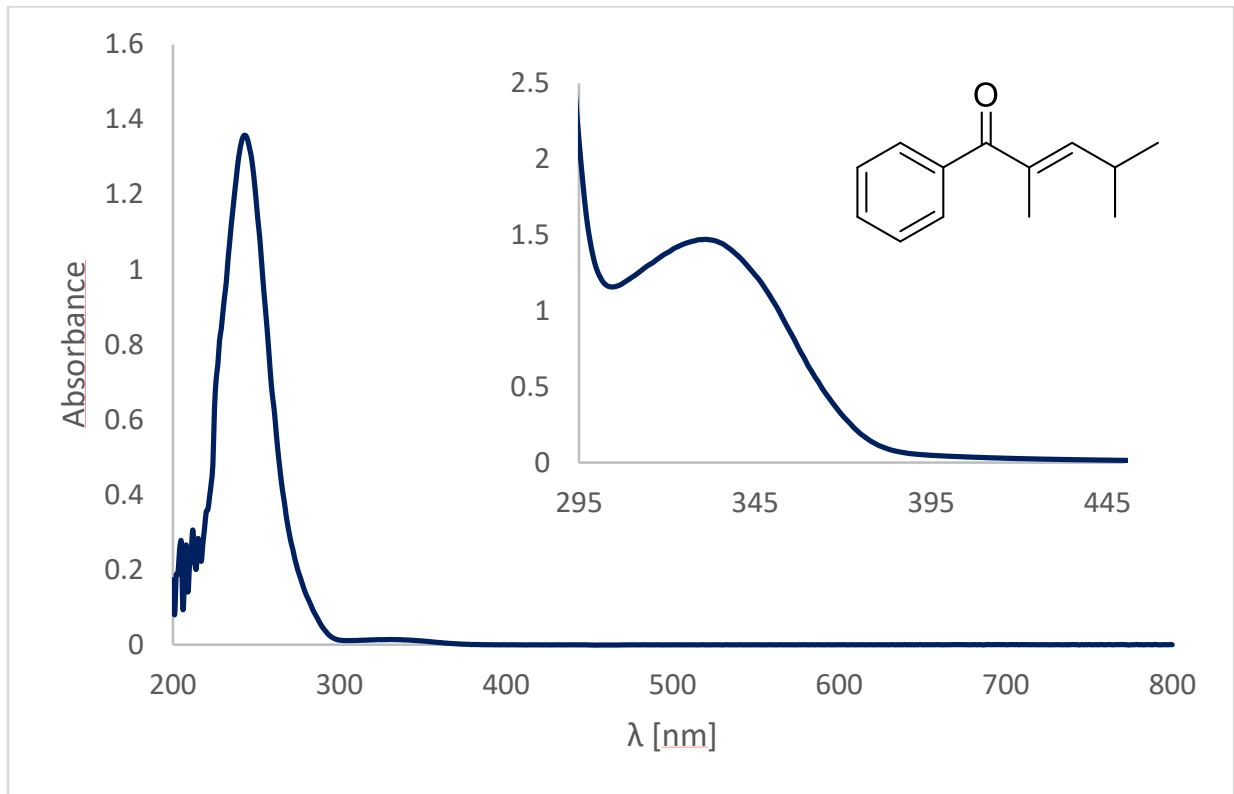

Figure S3: Absorption spectrum of 1 in $\mathrm{CH}_{2} \mathrm{Cl}_{2}$ at $0.1 \mathrm{mM}$; Inlet: at $10 \mathrm{mM}$.

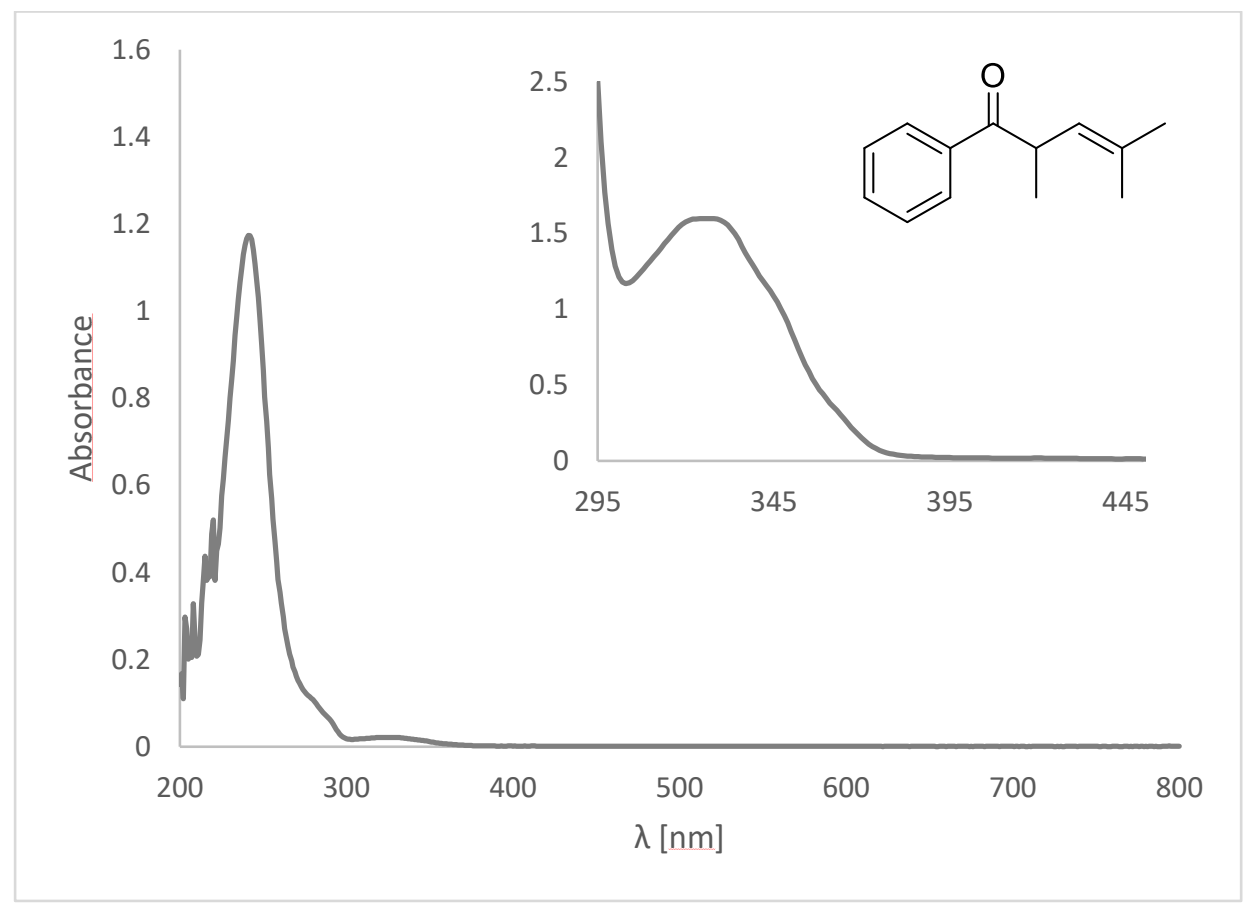

Figure S4: Absorption spectrum of $( \pm)-2$ in $\mathrm{CH}_{2} \mathrm{Cl}_{2}$ at $0.1 \mathrm{mM}$; Inlet: at $10 \mathrm{mM}$. 


\section{Reaction Monitoring}<smiles>CC(=CC(C)C)C(=O)c1ccccc1</smiles>

1

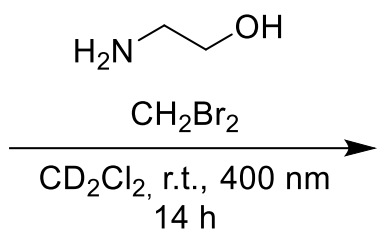

$14 \mathrm{~h}$<smiles>CC(C)=CC(C)C(=O)c1ccccc1</smiles>

2

In a round bottom flask under argon $1(94.1 \mathrm{mg}, 0.50 \mathrm{mmol}, 1.00$ eq. $)$, ethanolamine $(5.99 \mu \mathrm{L}$, $0.10 \mathrm{mmol}, 0.20$ eq.) and dibromomethane ( $17.5 \mu \mathrm{L}, 0.25 \mathrm{mmol}, 0.50$ eq.) were dissolved in degassed $\mathrm{CD}_{2} \mathrm{Cl}_{2}$. The reaction mixture was divided into 9 aliquots and transferred into screwcap vials with septum. The aliquots were irradiated at $400 \mathrm{~nm}$ at r.t. for $0,15,30,60,120,180$, 300, 420 and 540 min before being transferred into a NMR tube. The ${ }^{1} \mathrm{H}-\mathrm{NMR}$ yield of $(E)-\mathbf{1}$, (Z)-1 and $\mathbf{2}$ was determined by utilizing dibromomethane as internal standard and was corrected using the $\mathrm{t}=0 \mathrm{~min}$ datapoint as reference so that $[E]=100 \%$. The experiment was repeated and the average of both experiments for each datapoint was used for the graphics below and in the manuscript.

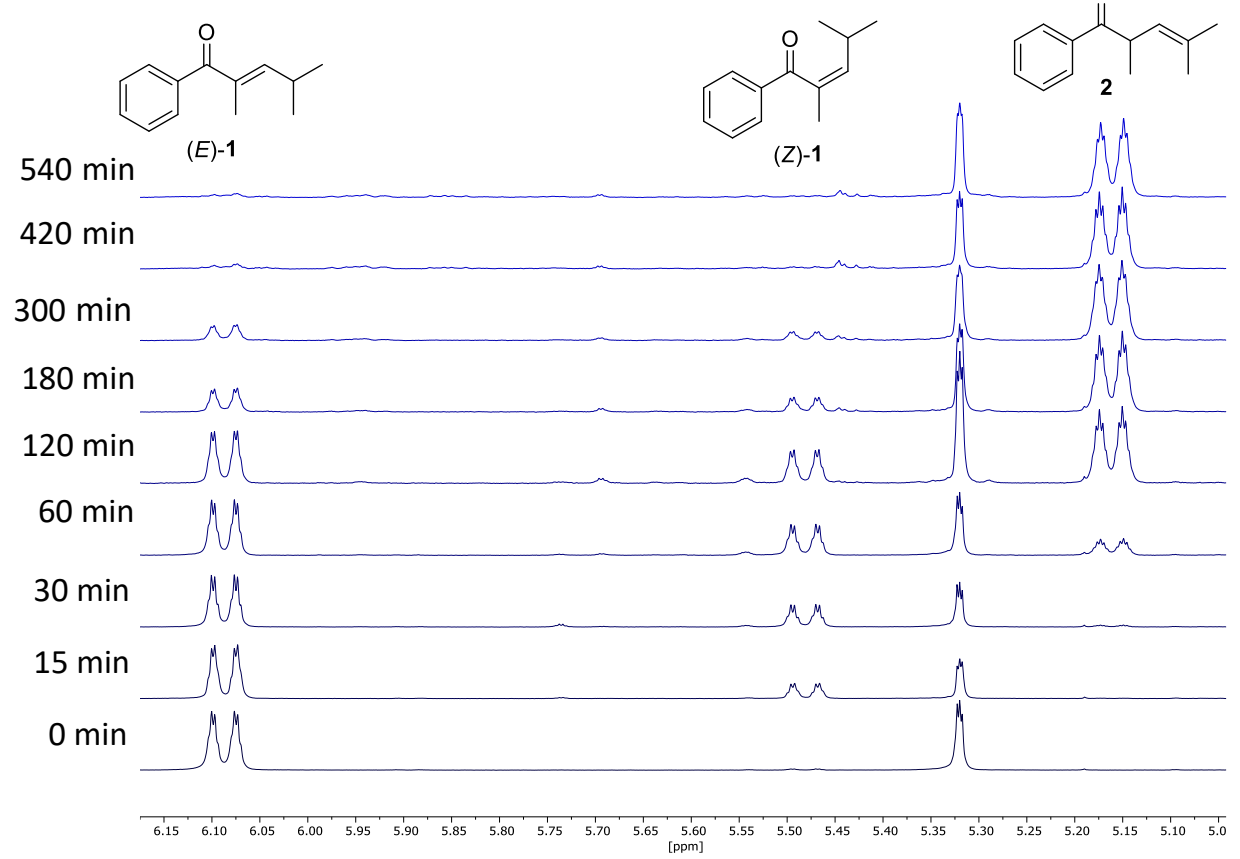

Figure S5: ${ }^{1} \mathrm{H}$ NMR spectra of the reaction mixture at different time points between 0 and $540 \mathrm{~min}$. For ${ }^{1} \mathrm{H}$ NMR data of the $Z$-isomer see reference. ${ }^{[11]}$ 
1. Experiment

\begin{tabular}{c|ccc|c} 
time [min] & E-1 [\%] & Z-1 [\%] & 2 [\%] & Z/E \\
\hline \hline $\mathbf{0}$ & 100 & 0 & 0 & 0.000 \\
$\mathbf{1 5}$ & 81 & 24 & 0 & 0.298 \\
$\mathbf{3 0}$ & 67 & 30 & 4 & 0.442 \\
$\mathbf{6 0}$ & 50 & 31 & 18 & 0.606 \\
$\mathbf{1 2 0}$ & 28 & 19 & 46 & 0.692 \\
$\mathbf{1 8 0}$ & 17 & 12 & 70 & 0.708 \\
$\mathbf{3 0 0}$ & 13 & 9 & 70 & 0.632 \\
$\mathbf{4 2 0}$ & 0 & 0 & 78 & \\
$\mathbf{5 4 0}$ & 0 & 0 & 76 & \\
\hline
\end{tabular}

2. Experiment

\begin{tabular}{c|ccc|c} 
time [min] & E-1 [\%] & Z-1 [\%] & 2 [\%] & Z/E \\
\hline \hline $\mathbf{0}$ & 100 & 0 & 0 & 0.000 \\
$\mathbf{1 5}$ & 78 & 23 & 0 & 0.301 \\
$\mathbf{3 0}$ & 63 & 29 & 3 & 0.461 \\
$\mathbf{6 0}$ & 50 & 30 & 8 & 0.600 \\
$\mathbf{1 2 0}$ & 33 & 22 & 38 & 0.667 \\
$\mathbf{1 8 0}$ & 19 & 13 & 66 & 0.652 \\
$\mathbf{3 0 0}$ & 15 & 10 & 68 & 0.667 \\
$\mathbf{4 2 0}$ & 0 & 0 & 76 & \\
$\mathbf{5 4 0}$ & 0 & 0 & 76 & \\
\hline
\end{tabular}

3. Average of $1+2$

\begin{tabular}{c|ccc|c} 
time [min] & E-1 [\%] & Z-1 [\%] & 2 [\%] & Z/E \\
\hline \hline $\mathbf{0}$ & 100 & 0 & 0 & 0.000 \\
$\mathbf{1 5}$ & 79 & 24 & 0 & 0.300 \\
$\mathbf{3 0}$ & 65 & 29 & 3 & 0.451 \\
$\mathbf{6 0}$ & 50 & 30 & 13 & 0.603 \\
$\mathbf{1 2 0}$ & 30 & 20 & 42 & 0.679 \\
$\mathbf{1 8 0}$ & 18 & 12 & 68 & 0.680 \\
$\mathbf{3 0 0}$ & 14 & 9 & 69 & 0.649 \\
$\mathbf{4 2 0}$ & 0 & 0 & 77 & \\
$\mathbf{5 4 0}$ & 0 & 0 & 76 & \\
\hline
\end{tabular}




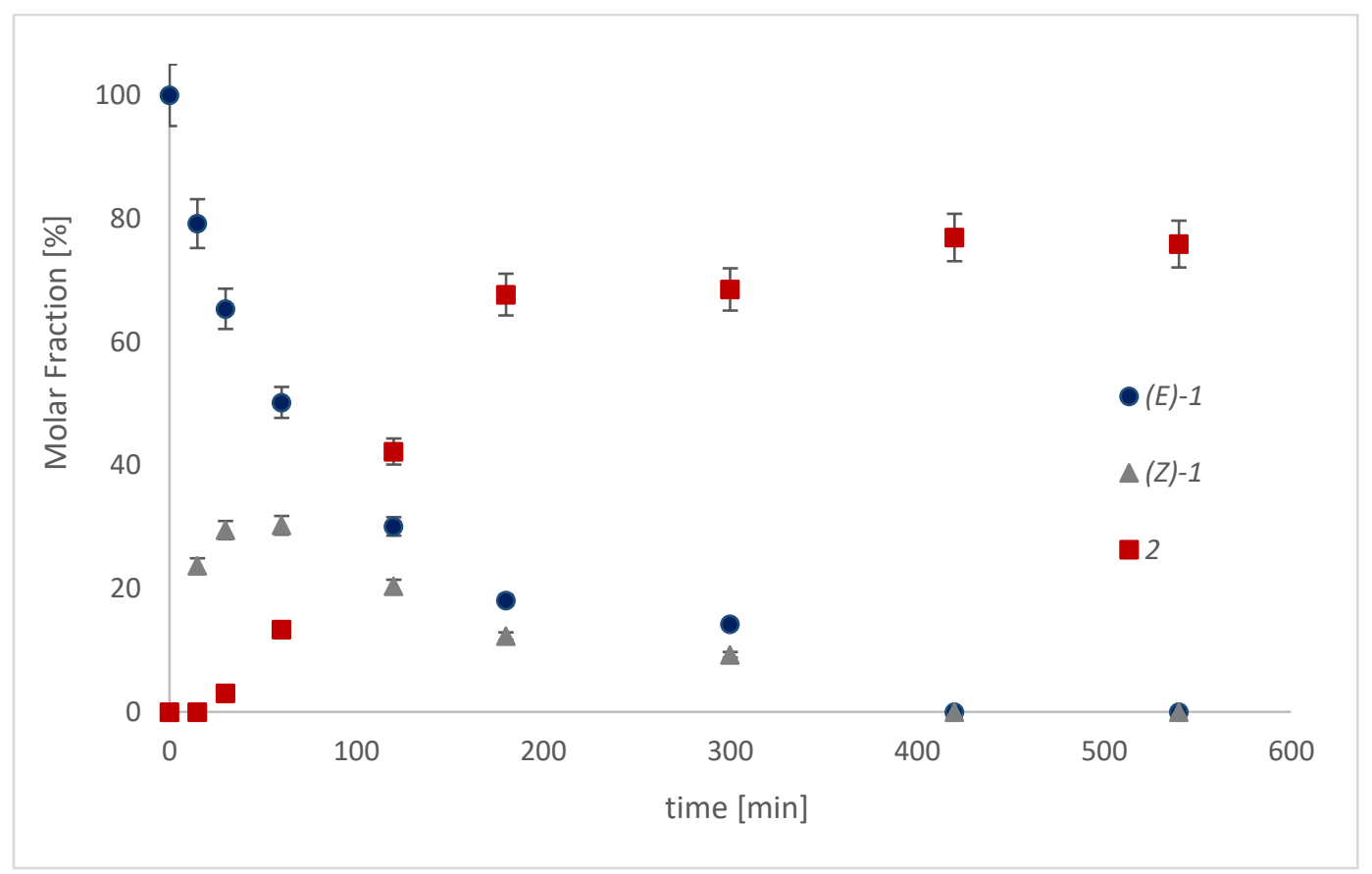

Figure S6: Reaction progress monitoring of the deconjugative isomerization of $\mathbf{1}$.

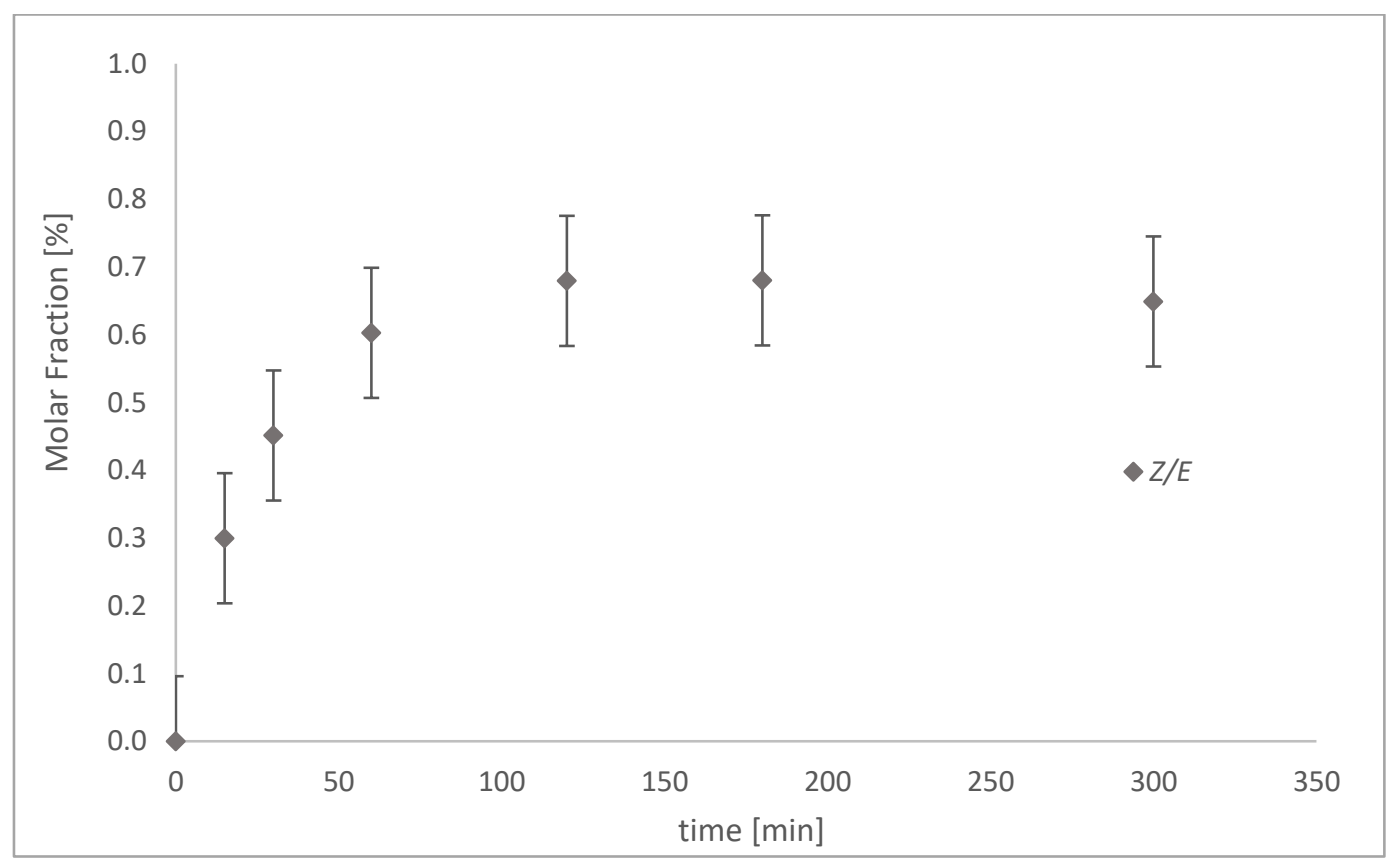

Figure S7: Progress of the $Z / E$-ratio over the course of the reaction. 


\section{Isotope labeling experiments}

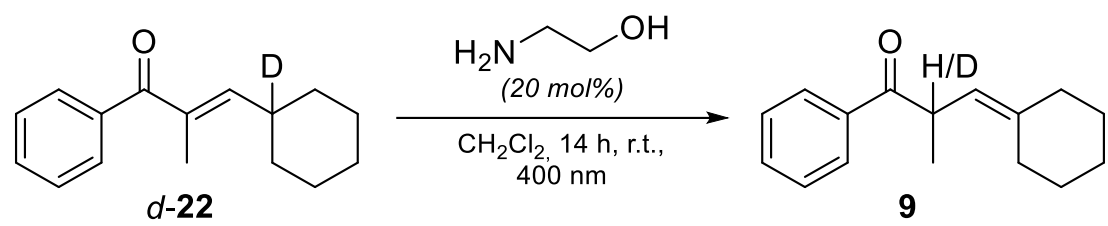

A screw-cap vial with septum under argon was charged with $d-22$ (45.9 $\mathrm{mg}, 0.20 \mathrm{mmol}$, 1.00 eq.), ethanolamine ( $2.40 \mu \mathrm{L}, 0.04 \mathrm{mmol}, 0.20$ eq. $)$ and the specified amount of $\mathrm{D}_{2} \mathrm{O}$. Subsequently, degassed dichloromethane $(4 \mathrm{~mL})$ was added, the vial was sealed and irradiated at $400 \mathrm{~nm}$ for $14 \mathrm{~h}$. Afterwards, the mixture was concentrated on silica and purified by column chromatography $\left(\mathrm{SiO}_{2}, n\right.$-pentane/ $\left.\mathrm{CH}_{2} \mathrm{Cl}_{2} 7: 3\right)$ to yield the product. Note: every reaction was repeated and yields given in the paper are the average of two independent experiments.

\begin{tabular}{c|c|cc|cc}
\multirow{2}{*}{ entry } & $\begin{array}{c}\mathbf{D}_{2} \mathbf{O} \\
{[\mathbf{m o l} \%]}\end{array}$ & $\begin{array}{c}\text { Yield } \\
{[\%]}\end{array}$ & $\begin{array}{c}1^{\text {st }} \text { reaction } \\
\text { incorporation } \\
{[\%]^{[\mathrm{a}]}}\end{array}$ & $\begin{array}{c}\text { Yield } \\
{[\%]}\end{array}$ & $\begin{array}{c}2^{\text {nd }} \\
\text { reaction } \\
\text { incorporation } \\
{[\%]^{[\mathrm{a}]}}\end{array}$ \\
\hline \hline $\mathbf{1}$ & 0 & 72 & $<5$ & 76 & $<5$ \\
$\mathbf{2}$ & 20 & 83 & 10 & 73 & 10 \\
$\mathbf{3}$ & 100 & 72 & 30 & 68 & 30 \\
\hline
\end{tabular}

${ }^{[a]}$ determined by ${ }^{1} \mathrm{H}-\mathrm{NMR}$ spectroscopy
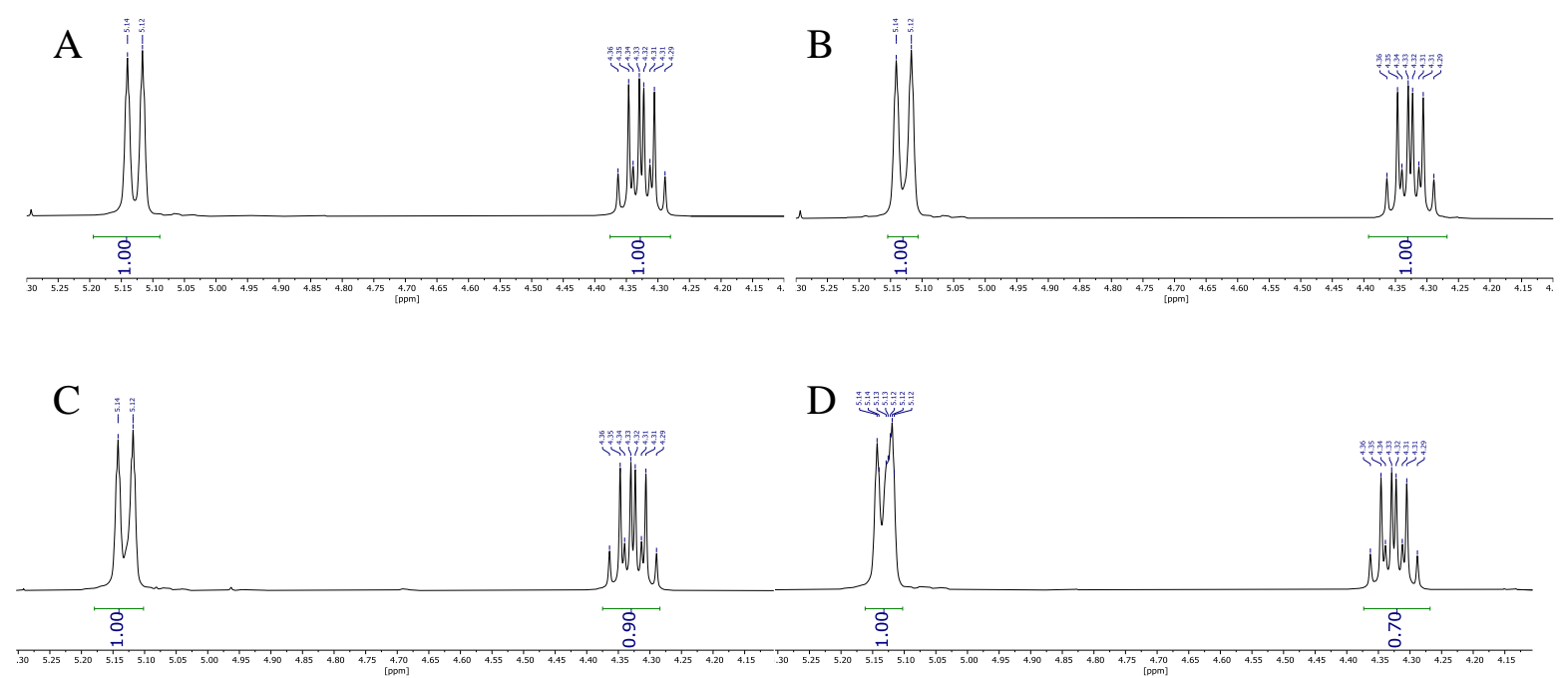

Figure S8: ${ }^{1} \mathrm{H}$ NMR spectra of ( \pm -9 after deconjugative isomerization of $d-22$ in the presence of varying amounts of $\mathrm{D}_{2} \mathrm{O}$. A: ${ }^{1} \mathrm{H}$ NMR spectrum of parent (土)-9; B: ${ }^{1} \mathrm{H}$ NMR spectrum of entry 1 ; C: ${ }^{1} \mathrm{H}$ NMR spectrum of entry 2; D: ${ }^{1} \mathrm{H}$ NMR spectrum of entry 3. 


\section{Kinetic isotope effect}<smiles>C/C(=C\C1(O)CCCCC1)C(=O)c1ccccc1</smiles>

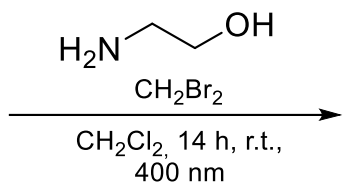<smiles>CC(O)(C(=O)c1ccccc1)C1CCCCC1</smiles>

9

In a round bottom flask under argon 22 or $d-22(0.25 \mathrm{mmol}, 1.00$ eq. $)$, ethanolamine $(2.99 \mu \mathrm{L}$, $0.05 \mathrm{mmol}, 0.20$ eq.) and dibromomethane $(8.74 \mu \mathrm{L}, 0.13 \mathrm{mmol}, 0.50$ eq.) were dissolved in degassed $\mathrm{CD}_{2} \mathrm{Cl}_{2}$. The reaction mixture was divided into 5 aliquots and transferred into screwcap vials with septum. The aliquots were irradiated at $400 \mathrm{~nm}$ at r.t. for 0, 30, 60, 90 and 120 min before being transferred into a NMR tube. The ${ }^{1} \mathrm{H}-\mathrm{NMR}$ yield of the corresponding $E$ and $Z$ isomer and ( \pm )-9 was determined by utilising dibromomethane as internal standard and was corrected using the $\mathrm{t}=0 \mathrm{~min}$ datapoint as reference so that $[E]=100 \%$. The experiment was repeated and the average of both experiments for each datapoint was used for the graphics below and in the manuscript.

Monitoring of $d-22$<smiles>[2H]C1(/C=C(\C)C(=O)c2ccccc2)CCCCC1</smiles>

d-22

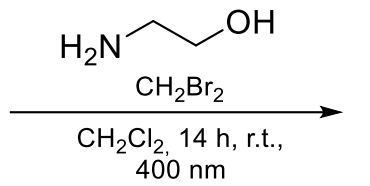
$400 \mathrm{~nm}$<smiles>CC(C=C1CCCCC1)C(=O)c1ccccc1</smiles>

9

\begin{tabular}{|c|c|c|c|c|c|c|}
\hline & \multicolumn{5}{|c|}{ time $[\mathrm{min}]$} \\
\hline & & 0 & 30 & 60 & 90 & 120 \\
\hline \multirow{3}{*}{$\begin{array}{c}1 . \\
\text { Experiment }\end{array}$} & $( \pm)-9$ [\%] & 0 & 7 & 23 & 31 & 39 \\
\hline & $E-22[\%]$ & 100 & 75 & 46 & 36 & 37 \\
\hline & $Z-22$ [\%] & 0 & 36 & 33 & 28 & 35 \\
\hline \multirow{3}{*}{$\begin{array}{c}2 . \\
\text { Experiment }\end{array}$} & $( \pm)-9[\%]$ & 0 & 5 & 18 & 32 & 38 \\
\hline & $E-22[\%]$ & 100 & 66 & 48 & 36 & 35 \\
\hline & $Z-22$ [\%] & 0 & 32 & 32 & 28 & 29 \\
\hline \multirow{3}{*}{$\varnothing$} & $( \pm)-9[\%]$ & 0 & 6 & 21 & 32 & 39 \\
\hline & $E-22[\%]$ & 100 & 70 & 47 & 36 & 36 \\
\hline & Z-22 [\%] & 0 & 34 & 33 & 28 & 32 \\
\hline
\end{tabular}




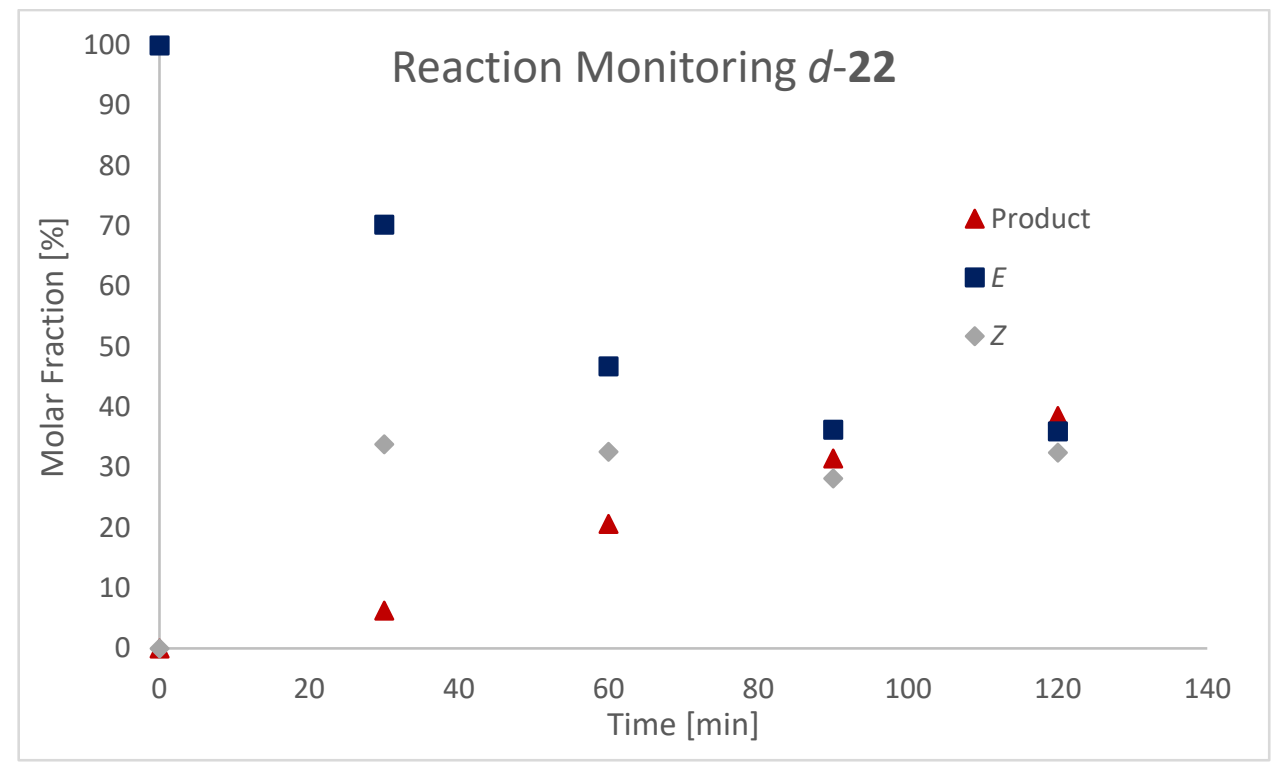

Figure S9: Reaction progress of the deconjugative isomerization of $d \mathbf{- 2 2}$.

Monitoring of 22<smiles>[Z20]C(=CC1CCCCC1)C(=O)c1ccccc1</smiles>

\begin{tabular}{|c|c|c|c|c|c|c|}
\hline & & \multicolumn{5}{|c|}{ time [min] } \\
\hline & & 0 & 30 & 60 & 90 & 120 \\
\hline \multirow{3}{*}{$\begin{array}{c}1 . \\
\text { Experiment }\end{array}$} & $( \pm)-9$ [\%] & 0 & 3 & 14 & 28 & 36 \\
\hline & $E-22[\%]$ & 100 & 73 & 43 & 29 & 24 \\
\hline & Z-22 [\%] & 0 & 31 & 32 & 25 & 25 \\
\hline \multirow{3}{*}{$\begin{array}{c}2 . \\
\text { Experiment }\end{array}$} & $( \pm)-9$ [\%] & 0 & 5 & 17 & 20 & 35 \\
\hline & $E-22$ [\%] & 100 & 66 & 44 & 42 & 26 \\
\hline & Z-22 [\%] & 0 & 33 & 32 & 32 & 25 \\
\hline \multirow{3}{*}{$\varnothing$} & $( \pm)-9$ [\%] & 0 & 4 & 16 & 24 & 35 \\
\hline & $E-22$ [\%] & 100 & 70 & 44 & 36 & 25 \\
\hline & Z-22 [\%] & 0 & 32 & 32 & 28 & 25 \\
\hline
\end{tabular}




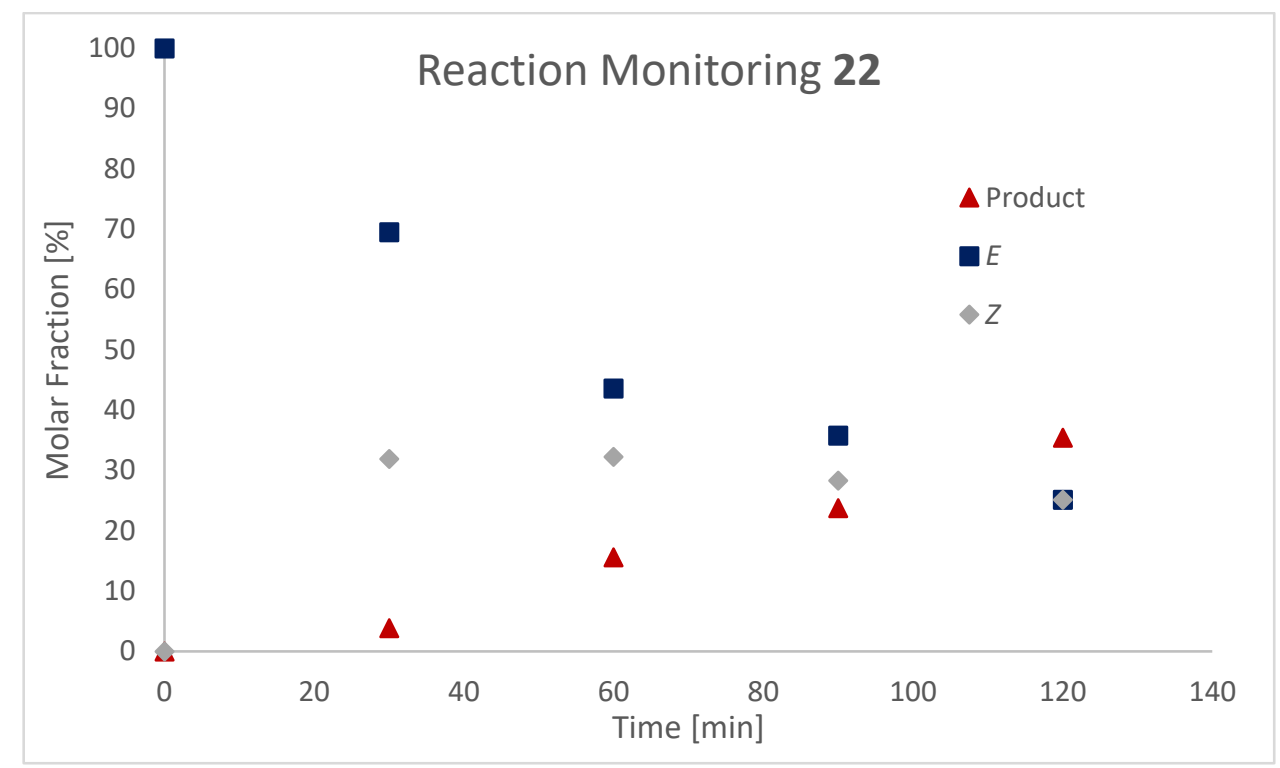

Figure S10: Reaction progress of the deconjugative isomerization of 22.

Determination of the kinetic isotope effect (KIE) is achieved by plotting the linear region of product formation of both reactions and comparison of the rates:

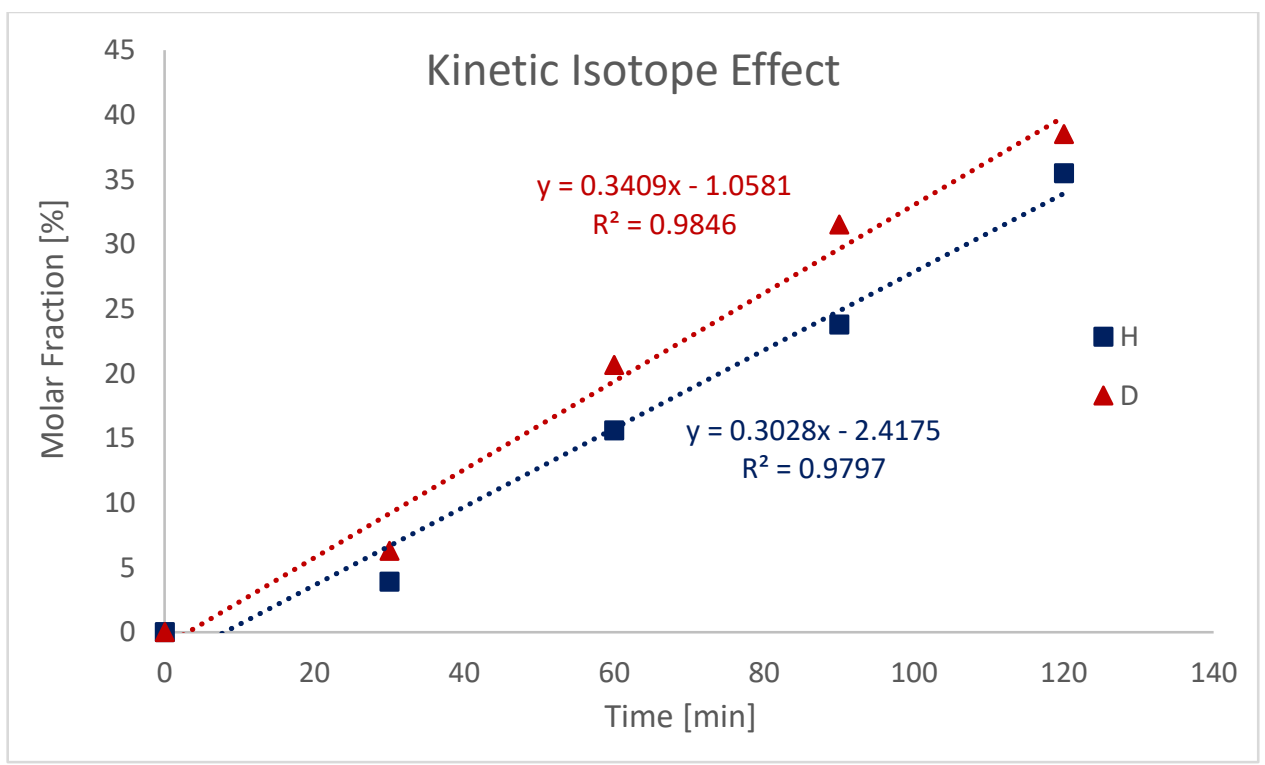

Figure S11: Plot of the rate of formation of ( \pm )-9 from 22 (blue) and $d-\mathbf{2 2}$ (red).

$$
\text { KIE: } \quad \frac{k_{H}}{k_{D}}=0.9
$$

Note: During the reaction progress monitoring of $d-22$ via ${ }^{1} \mathrm{H}$ NMR spectroscopy, the alkene signal of the Z-isomer shows a change in multiplicity over time, indicating a 
deuterium/hydrogen exchange in the neighboring position as the reaction proceeds. However, no change of multiplicity is observed for the alkene signal of the $E$-isomer:<smiles>[2H]C1(/C=C(\C)C(=O)c2ccccc2)CCCCC1</smiles>

$d-(E)-22$

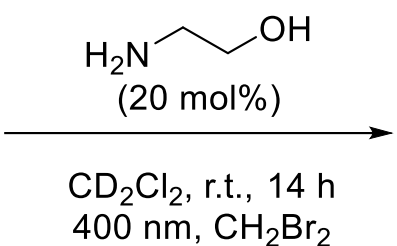

$400 \mathrm{~nm}, \mathrm{CH}_{2} \mathrm{Br}_{2}$<smiles>CC(C=C1CCCCC1)C(=O)c1ccccc1</smiles>

$( \pm)-9$

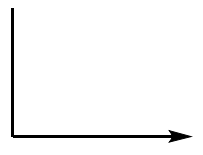<smiles>[R]C1(/C=C(/C)C(=O)c2ccccc2)CCCCC1</smiles>

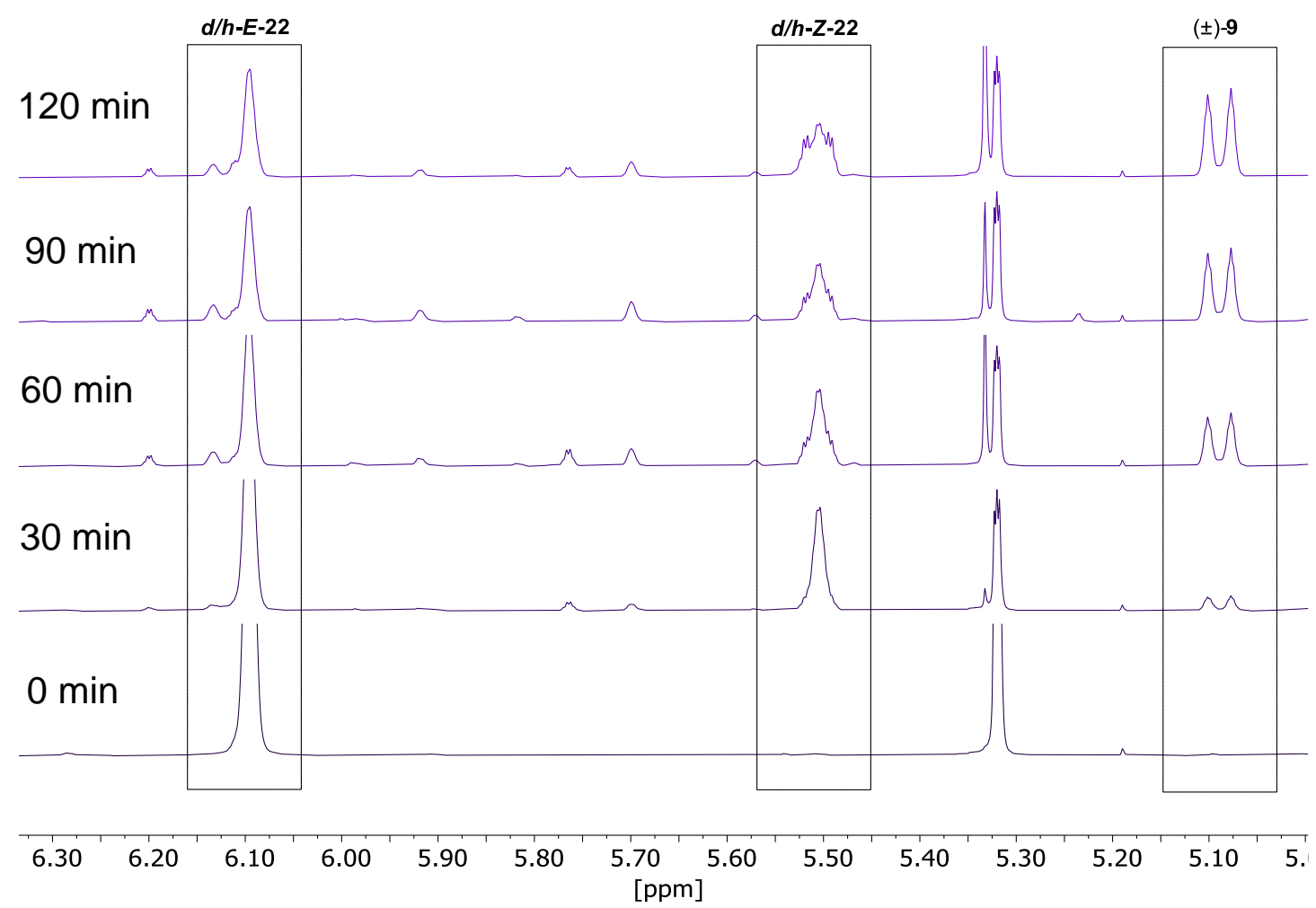

Figure S12: ${ }^{1} \mathrm{H}$ NMR spectrum of the reaction progress of $d-\mathbf{2 2}$. 
Hammett correlation for acyclic alkenes

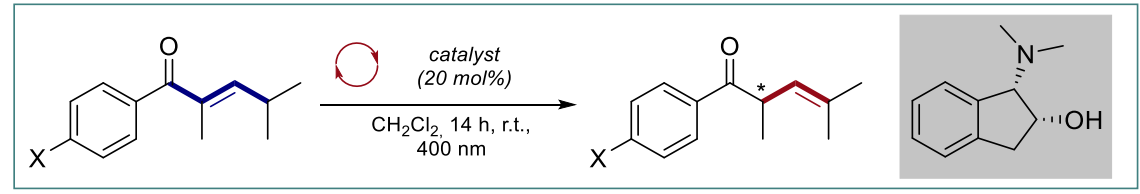

\begin{tabular}{c|c|c|c}
$\mathbf{X}$ & $\boldsymbol{\sigma}^{[12]}$ & e.r. & $\log ($ e.r. $)$ \\
\hline \hline $\mathrm{Br}$ & 0.232 & $54: 46$ & 0.07 \\
$\mathbf{F}$ & 0.062 & $59: 41$ & 0.15 \\
$\mathbf{H}$ & 0.000 & $64: 36$ & 0.25 \\
$\mathrm{Me}$ & -0.170 & $58: 42$ & 0.14 \\
OMe & -0.268 & $69: 31$ & 0.35 \\
\hline
\end{tabular}

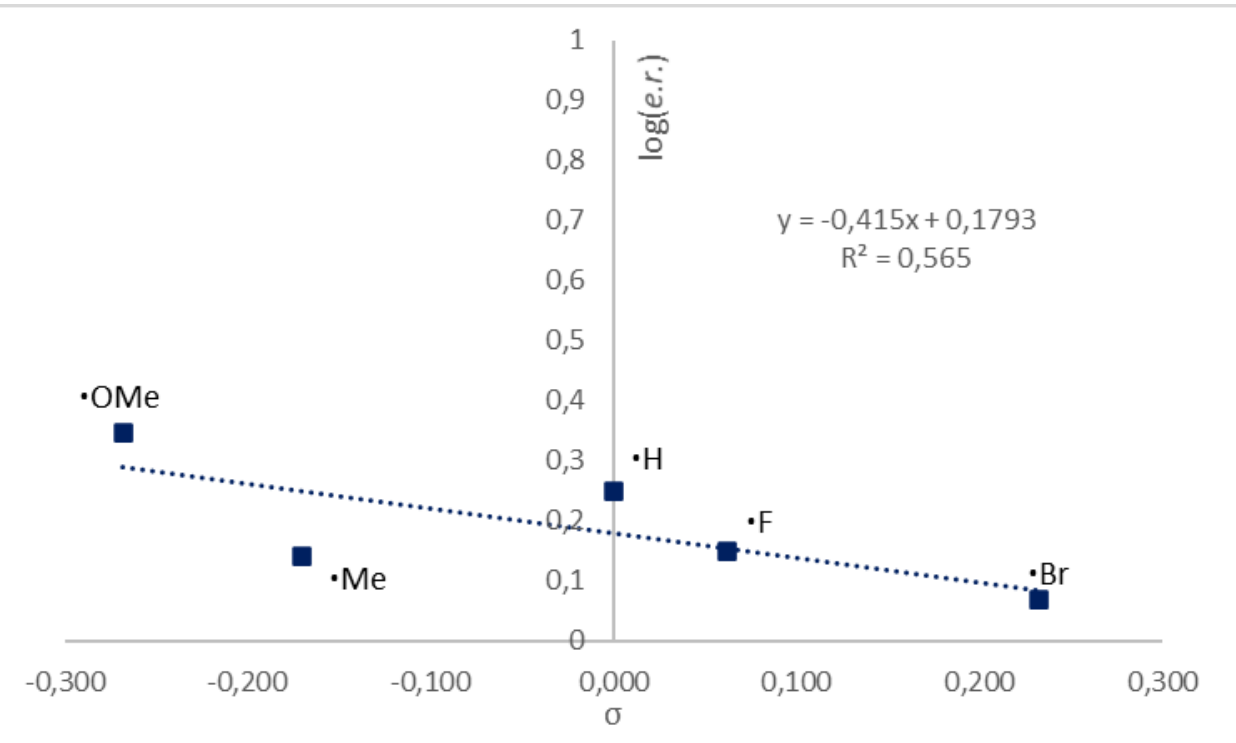

Figure S13: Hammett correlation for the enantioselective deconjugative isomerization of acyclic alkenes. 
Hammett correlation for cyclic alkenes

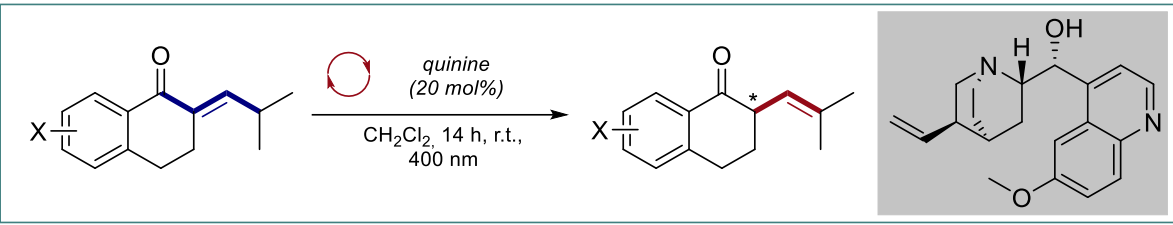

\begin{tabular}{c|c|c|c}
$\mathbf{X}$ & $\boldsymbol{\sigma}^{[12]}$ & e.r. & $\log ($ e.r. $)$ \\
\hline \hline $\boldsymbol{m}-\mathrm{Br}$ & 0.393 & $69: 31$ & 0.35 \\
$\boldsymbol{m}-\mathrm{F}$ & 0.337 & $82: 18$ & 0.66 \\
$\mathbf{H}$ & 0.000 & $92: 8$ & 1.06 \\
$\boldsymbol{p}-\mathrm{OMe}$ & -0.268 & $93: 7$ & 1.12 \\
\hline
\end{tabular}

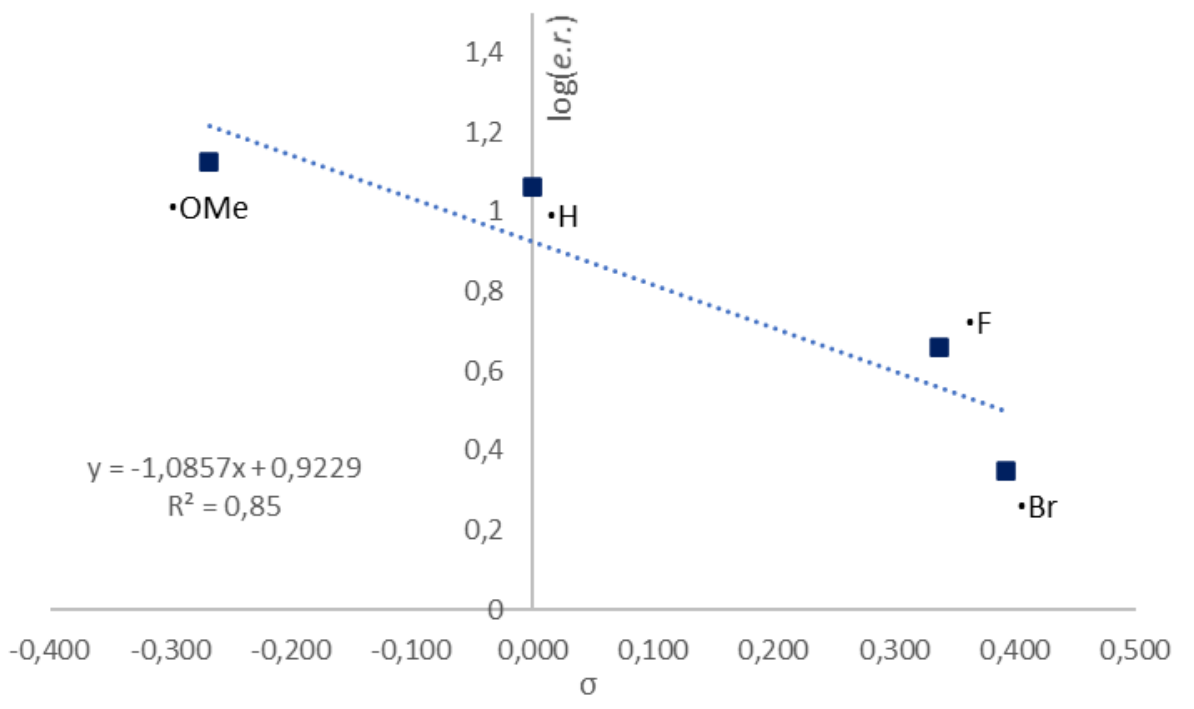

Figure S14: Hammett correlation for the enantioselective deconjugative isomerization of cyclic alkenes. 
CD-Spectrum of Compound (-)-12

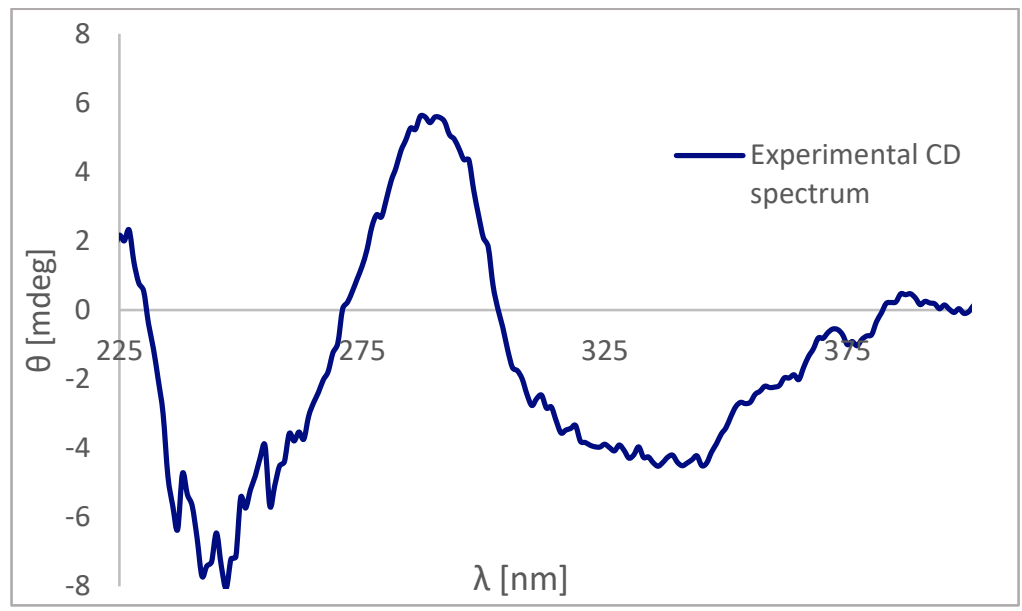

Figure 15: CD-spectrum of (-)-12 in $\mathrm{CH}_{2} \mathrm{Cl}_{2}(\mathrm{c}=0.33 \mathrm{mg} / \mathrm{mL})$. 


\section{DFT Calculations}

\section{Methods}

Geometry optimizations and energy evaluations were performed with the TURBOMOLE $7.5^{[13]}$ program. The structures were optimized without any geometry constraints using the TPSS meta-GGA functiona ${ }^{[14]}$ and an atom-pairwise dispersion correction (D3) ${ }^{[15]}$ A flexible triple zeta basis set (def2TZVP) ${ }^{[16]}$ was used in all calculations. For the calculation of free energy contributions of translation, rotations and harmonic vibrations $\left(\mathrm{G}^{\mathrm{RRHO}}{ }_{298}\right)$, a rotor approximation was applied for vibrational modes with wave numbers below $100 \mathrm{~cm}^{-1}{ }^{117]}$ Single point calculations were performed with the hybrid functional PW6B95(-D3). ${ }^{[18]}$ Free energies of solvation at $298 \mathrm{~K}\left(\mathrm{G}^{\text {solv }}{ }_{298}\right)$ were obtained with the COSMO-RS model ${ }^{[19]}$ using $\mathrm{CH}_{2} \mathrm{Cl}_{2}$ as solvent.

A conformational search of $\alpha$-isoprenyl substituted ketone XXX was conducted with the semiempirical tight-binding method GFN2-xTB, ${ }^{[20,21]}$ the resulting structures were refined with PW6B95-D3//TPSS-D3/def2-TZVP. We obtained three structurally distinct conformers which account for $99 \%$ of the total population. Electronic circular dichroism (CD) spectra of the conformers (Figure S18, Table S6) were obtained using the TDDFT algorithm with the range separated hybrid functional $\omega-\mathrm{B} 97 \mathrm{X}^{[22]}$ as implemented in ORCA. ${ }^{[23]}$ Boltzmann-weighted averaging of the three CD spectra (Figure S19) was done in a spreadsheet table.

To explain the observed stereochemical outcome in the formation of (-)-12, we have optimized several conformers of the $R e$ and $S i$ face complexes of the enol of $\mathbf{1 2}$ with cat. 12. In Figure S6, we report the most stable of each complexes which are mainly interacting by a hydrogen bond between the proton of the enol-OH group and the bridgehead nitrogen of quinine. The less stable $S i$ face complex further interacts with a $\mathrm{OH}-\pi$ bond, which is not present in the Re face complex. Instead, the latter obviously benefits from stabilizing vdW interactions between the isobutenyl and the quinoline groups. Assuming a protonation of the enol from the face opposite to the catalyst, the higher amount of the $R e$ complex would explain the formation of mainly (-)-12 with $(R)$ configuration.

\section{Results}

Table S4: Relative energies of intermediates in the isomerization of $\mathbf{1}$, calculated with DFT ${ }^{[a]}$.

\begin{tabular}{|c|c|c|c|c|c|}
\hline & \multirow{3}{*}{$\begin{array}{c}\text { E(TPSS-D3) } \\
{\left[\mathrm{E}_{\mathrm{h}}\right]}\end{array}$} & \multirow{3}{*}{$\begin{array}{c}\mathrm{E} \text { (PW6B95-D3) } \\
{\left[\mathrm{E}_{\mathrm{h}}\right]}\end{array}$} & \multicolumn{3}{|c|}{$\mathrm{G}^{\text {solv }}{ }_{298}$} \\
\hline & & & $\mathrm{G}^{\mathrm{RRHO}_{298}}$ & $\left(\mathrm{CH}_{2} \mathrm{Cl}_{2}\right)$ & $\begin{array}{c}\Delta G(298)_{\text {solv }}{ }^{[b]} \\
\left(\mathrm{CH}_{2} \mathbf{C l}_{2}\right)\end{array}$ \\
\hline & & & {$[\mathrm{kcal} / \mathrm{mol}]$} & [kcal/mol] & [kcal/mol] \\
\hline 1 & -580.58610367 & -581.20347934 & 132.435 & -11.611 & 0.00 \\
\hline 2 & -580.58434396 & -581.2032242 & 132.298 & -11.006 & 0.63 \\
\hline $\mathrm{Z}-1$ & -580.58390345 & -581.20141177 & 132.585 & -10.902 & 2.16 \\
\hline Dienol & -580.57235145 & -581.19270648 & 132.915 & -10.251 & 8.60 \\
\hline
\end{tabular}

[a] all calculations were performed with the def2-TZVP basis set, structures were optimized with TPSS-D3.

$[\mathrm{b}] \Delta \mathrm{G}(298)_{\text {solv }}=\Delta \mathrm{E}(\mathrm{PW} 6 \mathrm{~B} 95-\mathrm{D} 3 / / \mathrm{TPSS}-\mathrm{D} 3 /$ def2-TZVP $)+\Delta \mathrm{G}^{\mathrm{RRHO}}{ }_{298}+\Delta \mathrm{G}^{\mathrm{solv}_{298}}$ 
Table S5 Relative free energies and ratio of conformers of (-)-12, calculated with DFT ${ }^{[\mathrm{a}]}$.

\begin{tabular}{|c|c|c|c|c|c|c|}
\hline Conformer & E(TPSS-D3) & $\begin{array}{l}\text { E(PW6B95- } \\
\text { D3) }\end{array}$ & $\mathrm{G}^{\mathrm{RRHO}}{ }_{298}$ & $\begin{array}{c}\mathrm{G}^{\mathrm{solv}}{ }_{298} \\
\left(\mathrm{CH}_{2} \mathrm{Cl}_{2}\right)\end{array}$ & $\begin{array}{c}\Delta \mathbf{G}(\mathbf{2 9 8})_{\text {solv }}{ }^{[\mathrm{b}]} \\
\left(\mathrm{CH}_{2} \mathbf{C l}_{2}\right)\end{array}$ & $\mathbf{p i}_{\mathbf{i}}^{[\mathrm{lc}]}$ \\
\hline & {$\left[\mathrm{E}_{\mathrm{h}}\right]$} & {$\left[\mathrm{E}_{\mathrm{h}}\right]$} & {$[\mathrm{kcal} / \mathrm{mol}]$} & {$[\mathrm{kcal} / \mathrm{mol}]$} & [kcal/mol] & [\%] \\
\hline $\mathrm{C} 1$ & -618.7223339 & -619.3852996 & 137.748 & -12.112 & 0.00 & 13 \\
\hline $\mathrm{C} 2$ & -618.7218749 & -619.3853978 & 137.451 & -12.688 & -0.93 & 63 \\
\hline $\mathrm{C} 3$ & -618.7213840 & -619.3847988 & 137.260 & -12.307 & -0.37 & 24 \\
\hline
\end{tabular}

[a] all calculations were performed with the def2-TZVP basis set, structures were optimized with TPSS-D3.

[b] $\Delta \mathrm{G}(298)_{\text {solv }}=\Delta \mathrm{E}\left(\mathrm{PW} 6 \mathrm{~B} 95-\mathrm{D} 3 / / \mathrm{TPSS}-\mathrm{D} 3 /\right.$ def2-TZVP) $+\Delta \mathrm{G}^{\mathrm{RRHO}}{ }_{298}+\Delta \mathrm{G}^{\text {solv }_{298}}$

[c] Boltzmann-weighted ratio $\left(\mathrm{p}_{\mathrm{i}}=\exp \left(\Delta \mathrm{G}_{\mathrm{i}} /(\mathrm{RT})\right) / \Sigma_{\mathrm{j}}\left(\exp \left(\Delta \mathrm{G}_{\mathrm{j}} /(\mathrm{RT})\right)\right)\right.$ of conformer $i, j$ running over all conformers 

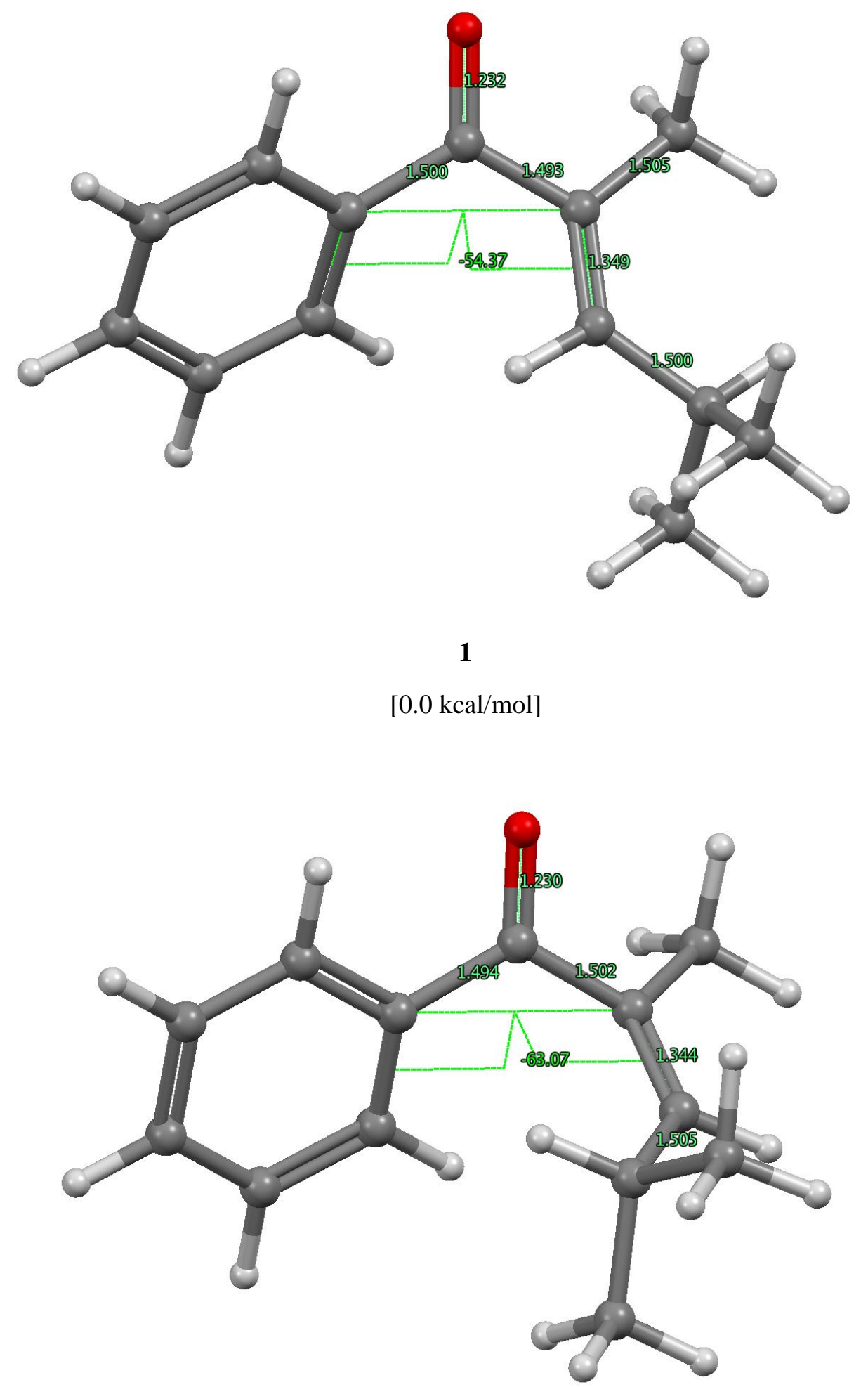

Z-1

[+2.2 kcal $/ \mathrm{mol}]$

Figure S16 Optimized molecular structures (TPSS-D3/def2-TZVP) of intermediates in the isomerization of 1. Interatomic distances as indicated in $\AA$, dihedral angles in degrees. In square brackets: relative free energies $\left(\Delta \mathrm{G}(298)_{\text {solv }}\right)$ in $\mathrm{kcal} / \mathrm{mol}$. 


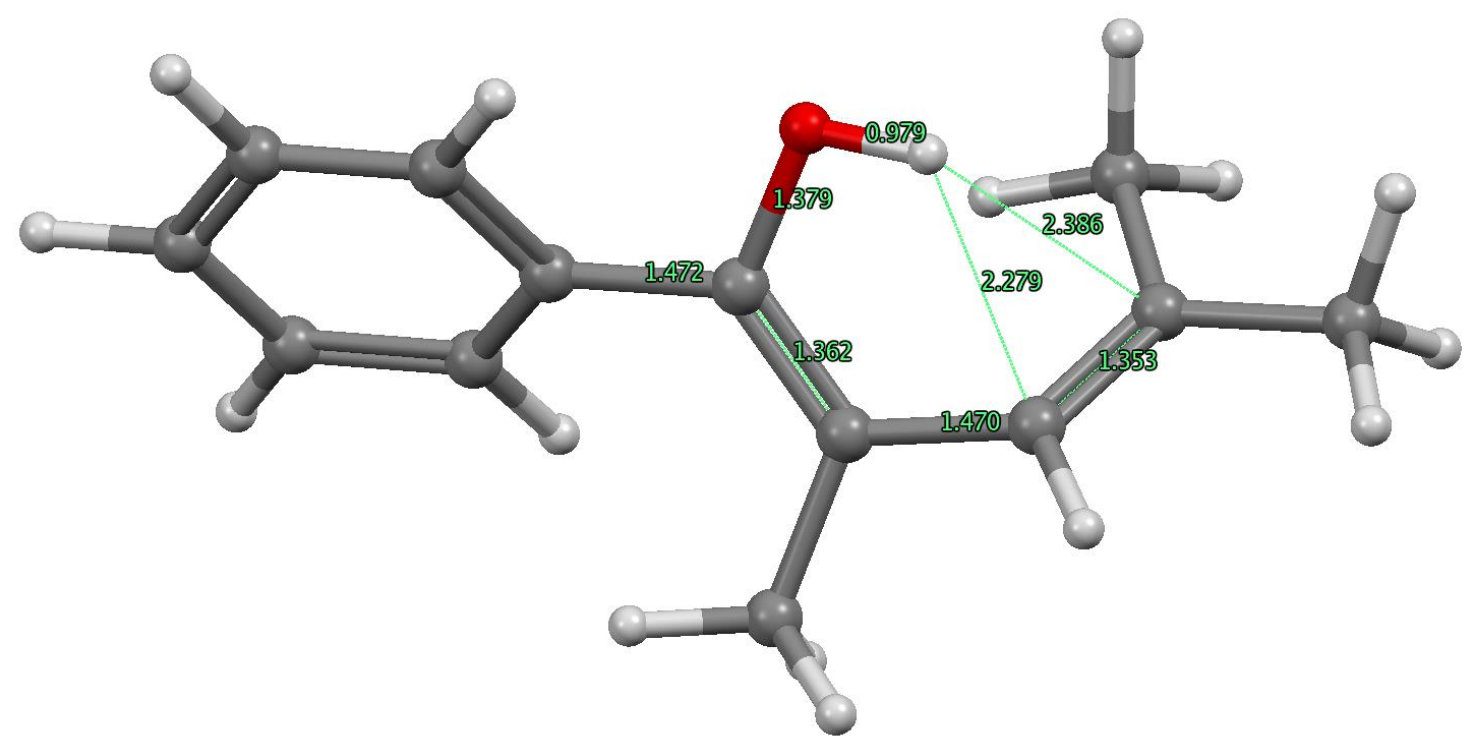

Dienol

$[+8.6 \mathrm{kcal} / \mathrm{mol}]$

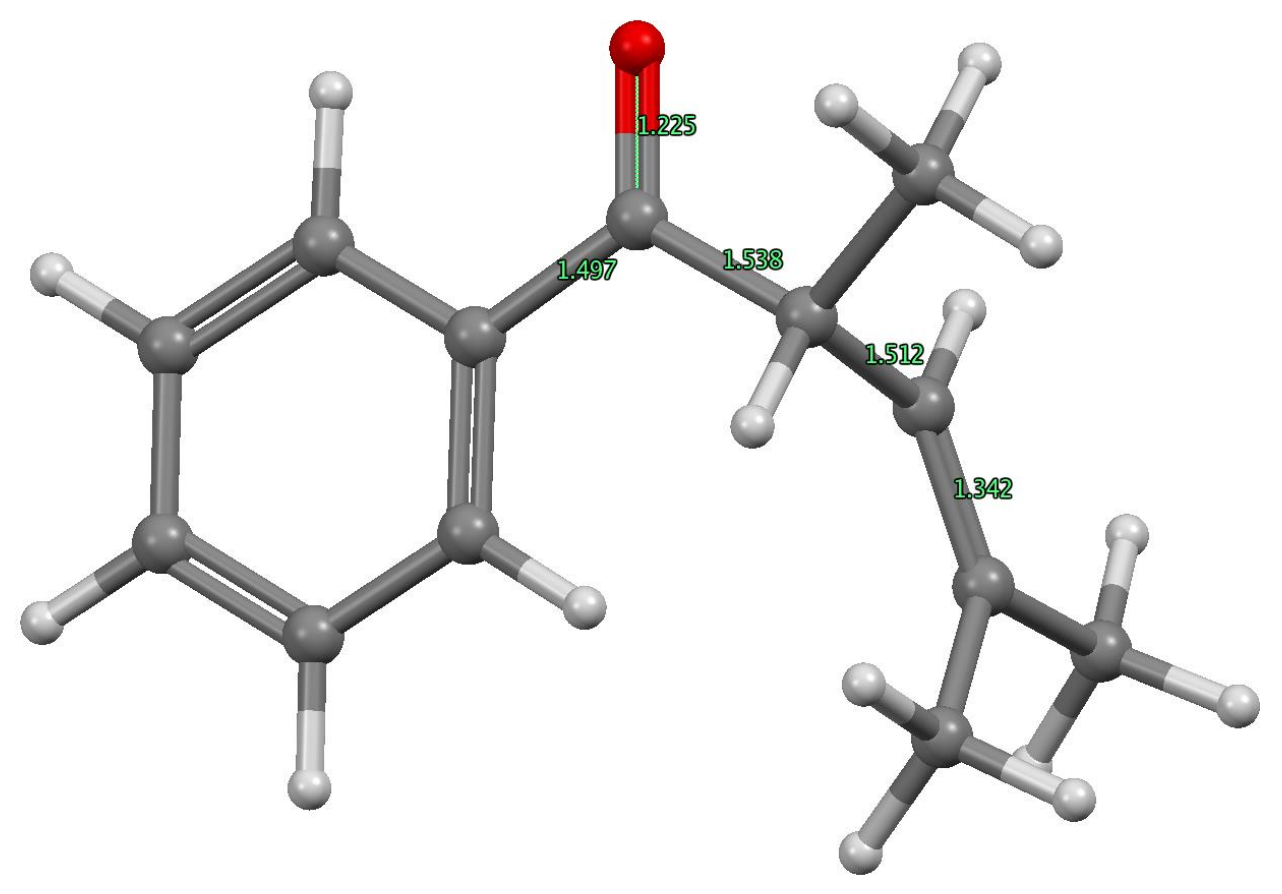

2

$[+0.6 \mathrm{kcal} / \mathrm{mol}]$

Figure S16 (continued) Optimized molecular structures (TPSS-D3/def2-TZVP) of intermediates in the isomerization of $\mathbf{1}$. Interatomic distances as indicated in $\AA$. In square brackets: relative free energies $\left(\Delta \mathrm{G}(298)_{\text {solv }}\right)$ in $\mathrm{kcal} / \mathrm{mol}$. 


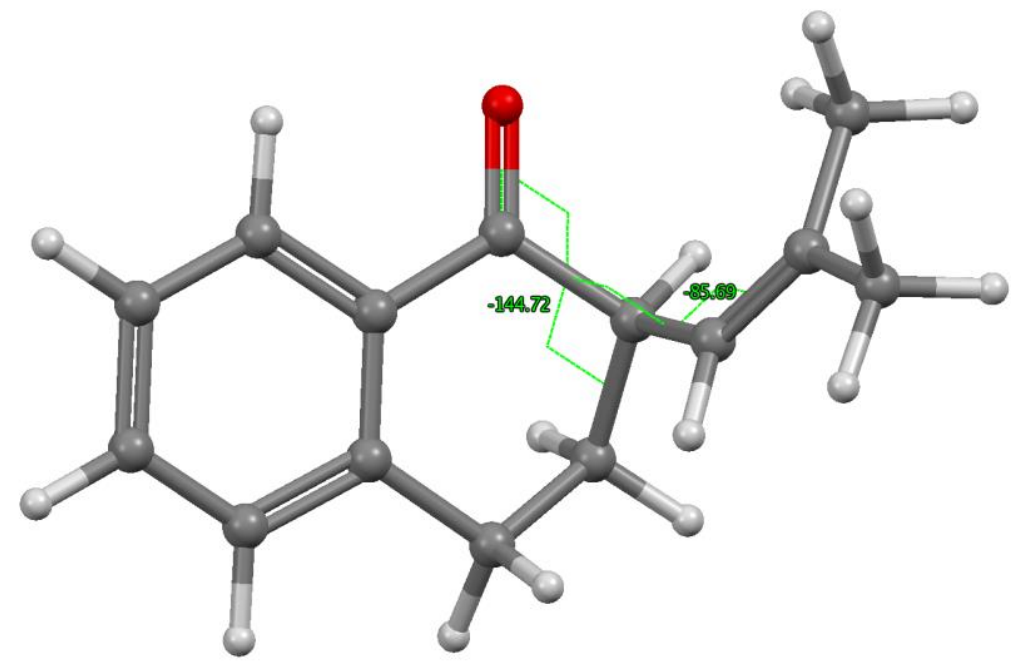

$\mathrm{C} 1[13 \%]$

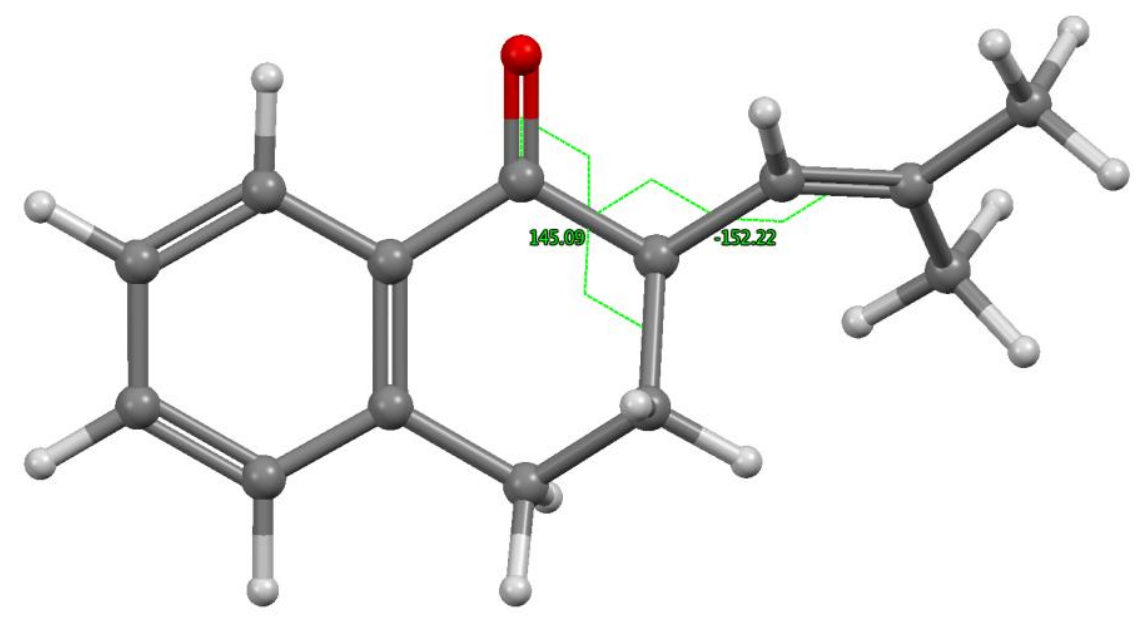

C2 [63\%]

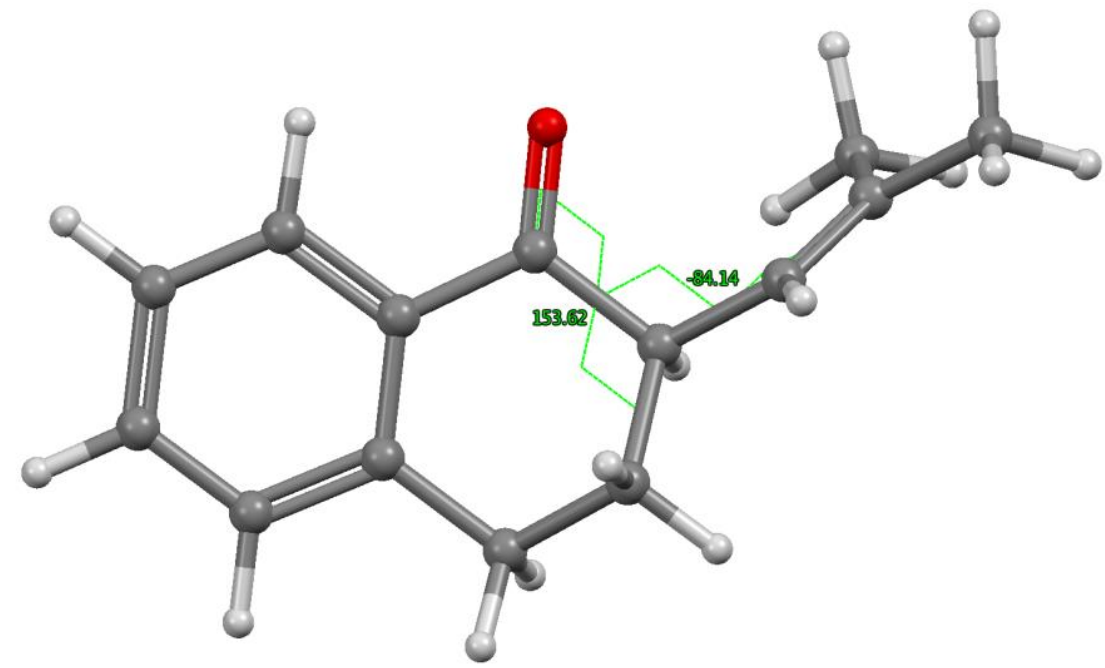

C3 [24\%]

Figure S17 Optimized molecular structures (TPSS-D3/def2-TZVP) of three dominating conformers of (-)-12. Dihedral angles as indicated in $\left[{ }^{\circ}\right]$. In square brackets: conformers equilibrium ratio. 


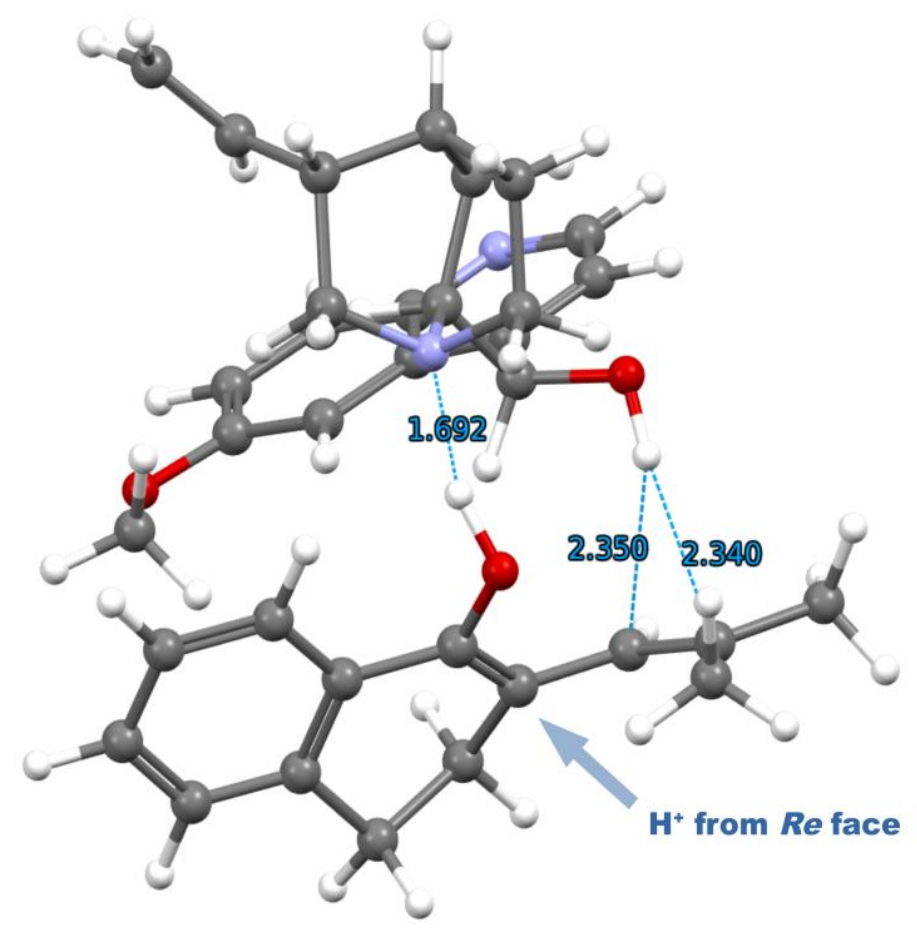

catalyst on the Si face of enol-12

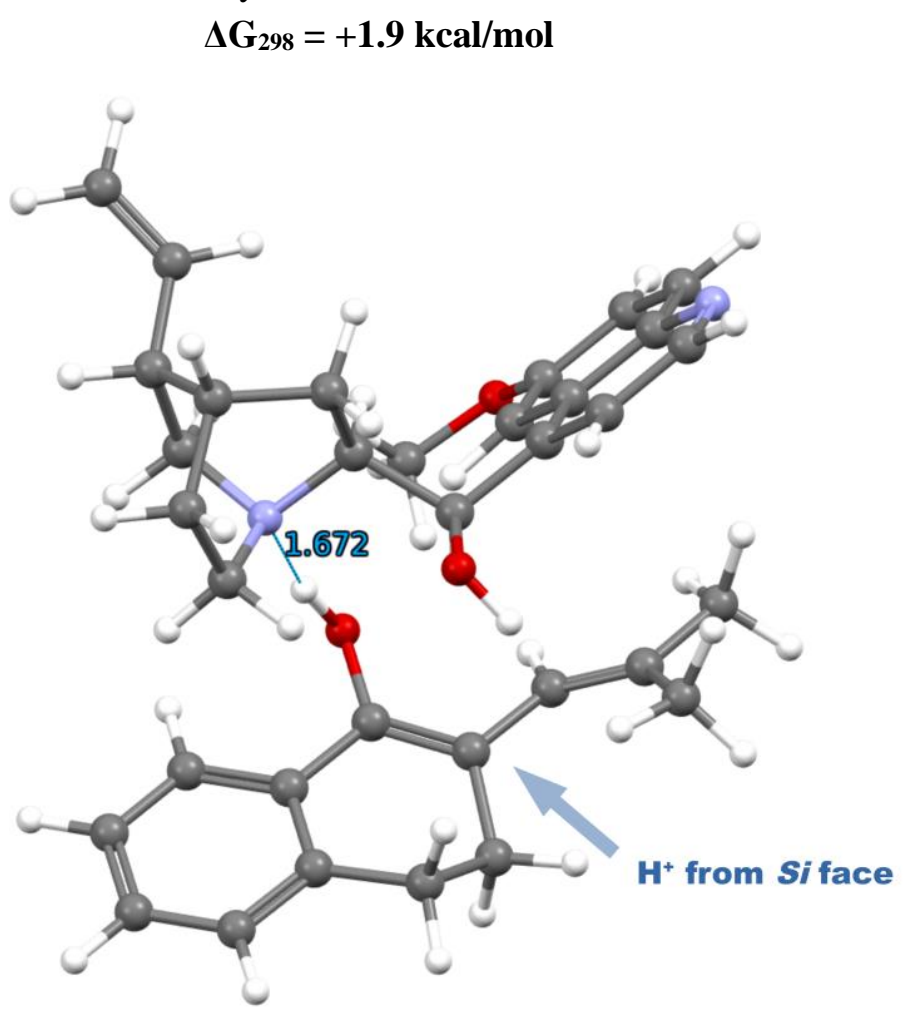

catalyst on the Re face of enol-12

$$
\Delta G_{298}=0.0 \mathrm{kcal} / \mathrm{mol}
$$

Figure S18: Optimized structures of the (most stable) complexes of the enol of $\mathbf{1 2}$ with catalyst cat 12. 


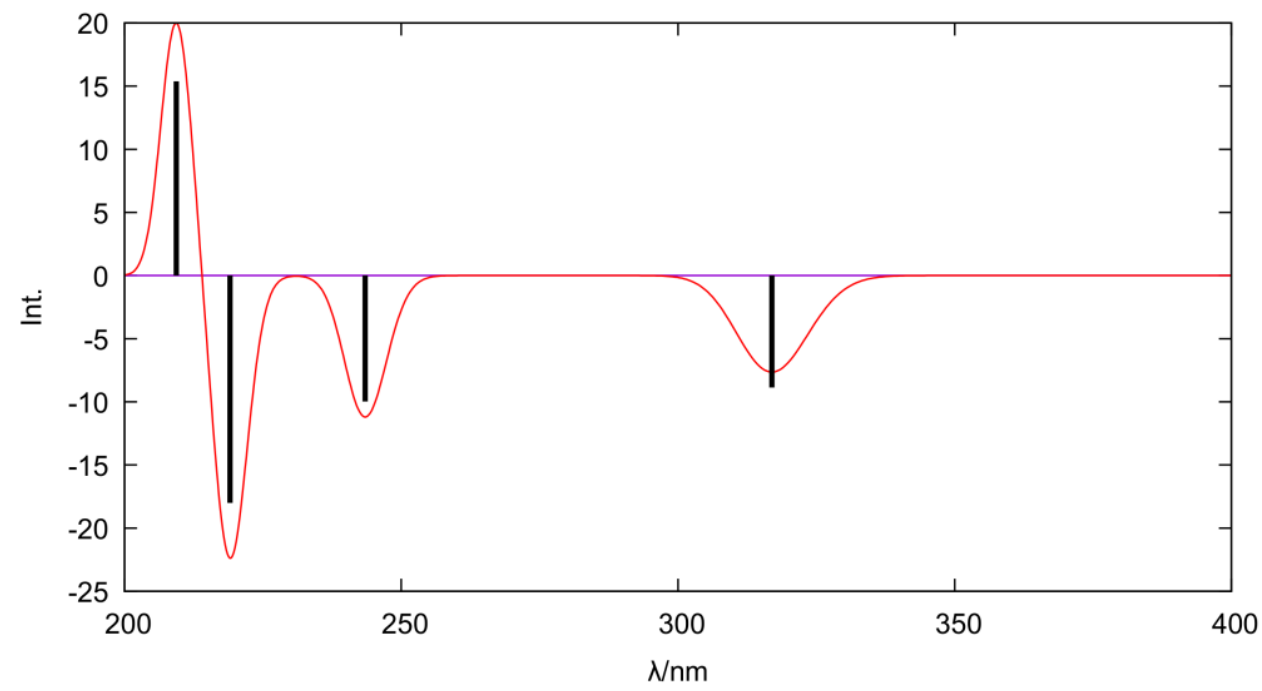

C1

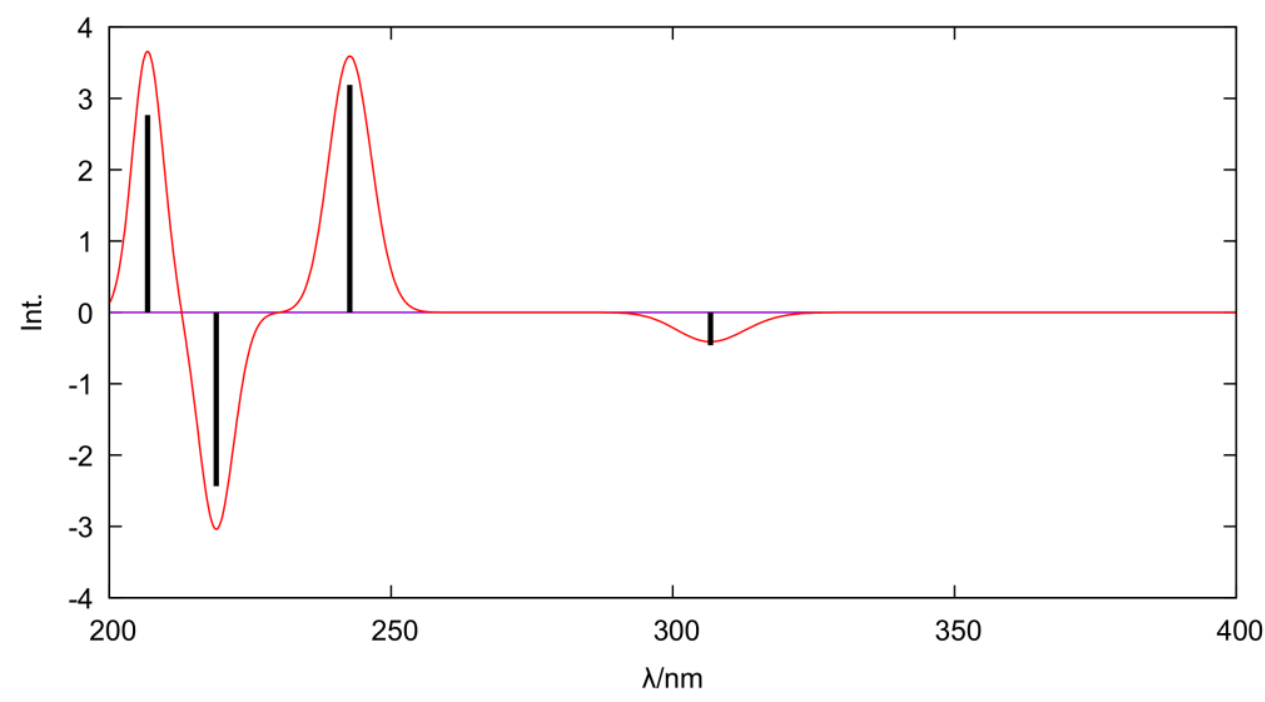

C2

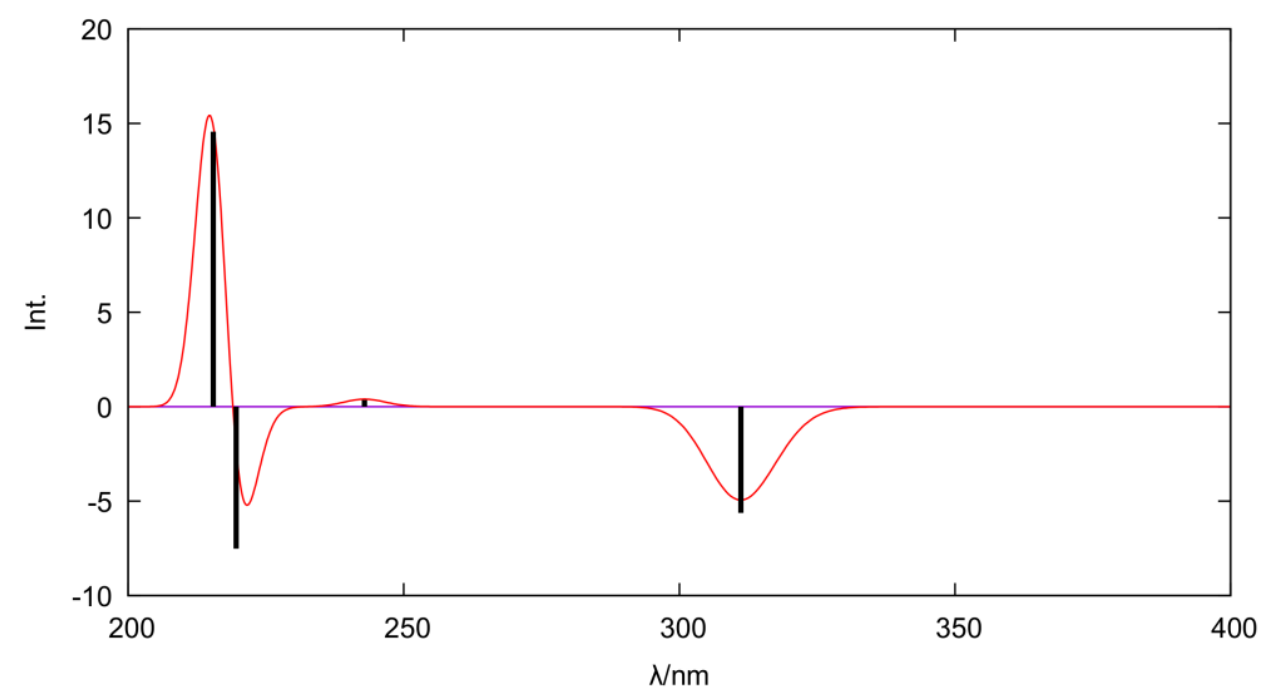

C3

Figure S18 Calculated CD spectra ( $\omega-B 97 X / d e f 2-T Z V P)$ of conformers of (-)-12. 
Table S6 CD intensities of the four transition with $\lambda>200 \mathrm{~nm}$ for conformers C1-C3 of (-)-12

\begin{tabular}{|c|c|c|c|c|c|c|}
\hline & $\begin{array}{l}\text { State Energy } \\
(\mathrm{cm}-1)\end{array}$ & $\begin{array}{l}\text { Wave } \\
(\mathrm{nm})\end{array}$ & $\begin{array}{l}\text { elength } \quad R \\
\left(1 \mathrm{e} 40^{*} \mathrm{cgs}\right)\end{array}$ & $\begin{array}{c}M X \\
\text { (au) }\end{array}$ & $\begin{array}{c}\text { MY } \\
(\mathrm{au}) \quad(\mathrm{au})\end{array}$ & MZ \\
\hline \multicolumn{7}{|l|}{ Conformer $\mathrm{C} 1$} \\
\hline & 131548.4 & 317.0 & -8.86129 & -0.07880 & -0.25811 & -0.29557 \\
\hline & 241070.0 & 243.5 & -9.97100 & -0.09511 & $1-0.12366$ & 0.17464 \\
\hline & 345647.4 & 219.1 & -17.99775 & -0.03966 & $6-0.18150$ & $0 \quad 0.09949$ \\
\hline & $4 \quad 47765.7$ & 209.4 & 15.37776 & 0.02002 & $2-0.14799$ & 90.06570 \\
\hline \multicolumn{7}{|l|}{ Conformer C2 } \\
\hline & 132605.5 & 306.7 & -0.46093 & -0.09352 & $2-0.35630$ & 0.06040 \\
\hline & 241205.0 & 242.7 & 3.18875 & 0.00831 & -0.00879 & 0.21721 \\
\hline & 345664.9 & 219.0 & -2.43445 & -0.00172 & $2-0.01772$ & 0.22880 \\
\hline & $4 \quad 48357.7$ & 206.8 & 2.76628 & -0.03174 & -0.02676 & -0.04109 \\
\hline \multicolumn{7}{|l|}{ Conformer C3 } \\
\hline & 132138.9 & 311.1 & -5.62825 & -0.17575 & $5-0.29814$ & 0.07512 \\
\hline & 241172.9 & 242.9 & 0.35316 & -0.00948 & $3-0.03494$ & -0.21348 \\
\hline & 345538.5 & 219.6 & -7.52172 & -0.00173 & $3-0.06574$ & 0.16709 \\
\hline & $4 \quad 46416.2$ & 215.4 & 14.56126 & -0.01576 & $6-0.13490$ & $\begin{array}{ll}0 & -0.03442\end{array}$ \\
\hline
\end{tabular}

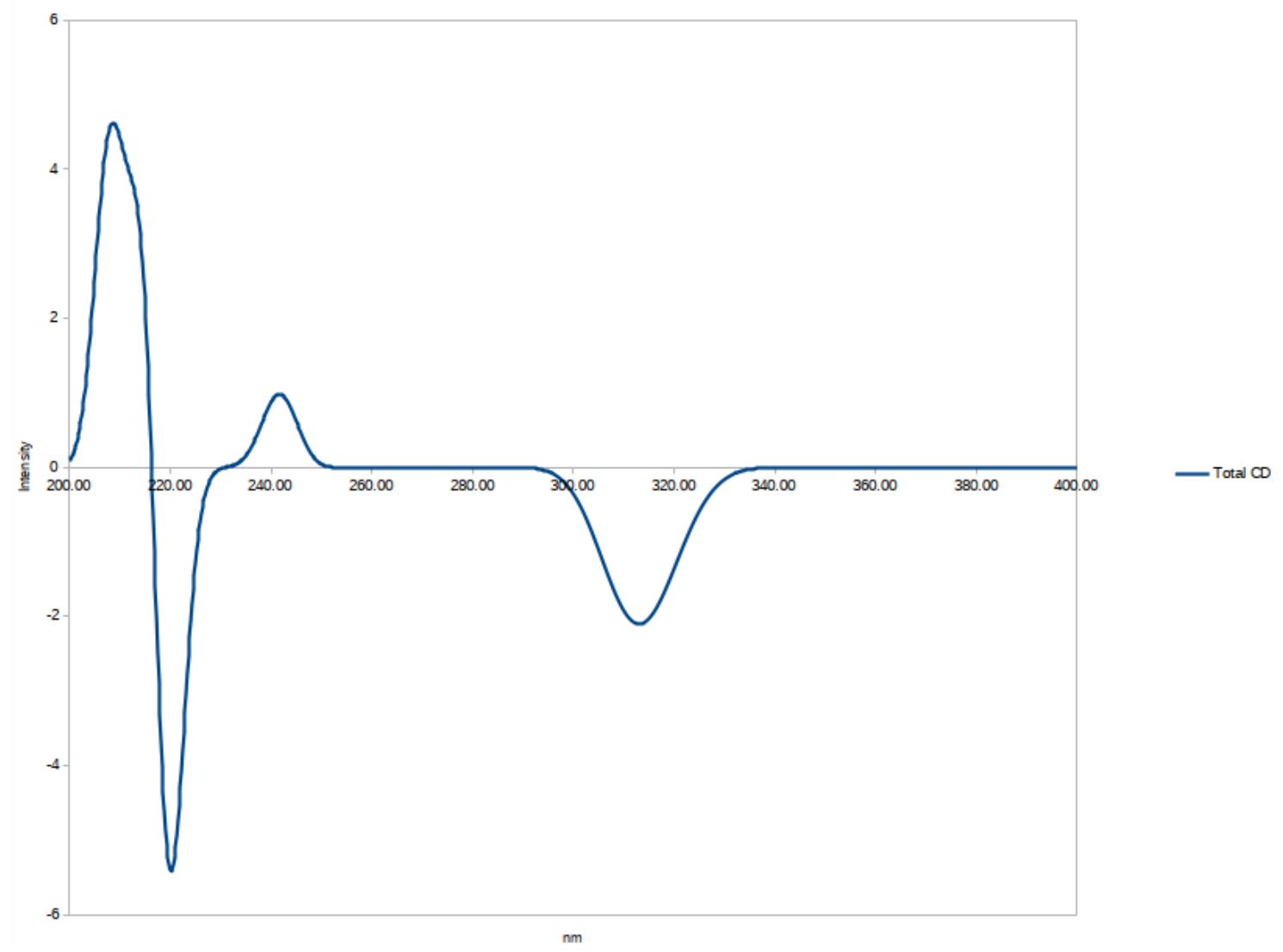

Figure S19 Boltzmann-averaged CD spectrum ( $\omega$-B97X/def2-TZVP), taking three conformers of XXX into consideration. 


\section{DFT optimized (TPSS-D3/def2-TZVP) cartesian coordinates}

\section{1}

$\mathrm{E}(\mathrm{TPSS}-\mathrm{D} 3 / \operatorname{def} 2-\mathrm{TZVP})=-580.5861036747$ (conv)

Lowest Freq. = $24.97 \mathrm{~cm}^{\wedge}-1$

30

1 (003s000/opt)

C $-\quad-2.9606588$

C $\quad-4.0103546$

C $\quad-4.0020368$

C $\quad-2.9387555$

C $\quad-1.8739573$

C $\quad-1.8731740$

C $\quad-0.7955947$

O $\quad-1.0897187$

C $\quad 0.6246296$

C $\quad 1.0511688$

C $\quad 2.4435215$

H $\quad 3.1236338$

C $\quad 2.9344509$

$\mathrm{H} \quad 2.9559494$

$\mathrm{H} \quad 3.9433080$

$\mathrm{H} \quad 2.2717361$

C $\quad 2.4654777$

$\mathrm{H} \quad 2.1564801$

$\mathrm{H} \quad 3.4735175$

$\mathrm{H} \quad 1.7849563$

$\mathrm{H} \quad 0.3136071$

C $\quad 1.4992327$

$\mathrm{H} \quad 2.5429728$

$\mathrm{H} \quad 1.4340578$

$\mathrm{H} \quad 1.1448502$

H $\quad-1.0515982$

$\mathrm{H} \quad-2.9394578$

$\mathrm{H} \quad-4.8265928$

$\mathrm{H} \quad-4.8401544$

$\mathrm{H} \quad-2.9614964$

$-0.9747674$

$-0.0767030$

1.1411142

1.4559505

0.5673934

$-0.6543148$

$-1.6817246$

$-2.8777059$

$-1.2654935$

$-0.0932245$

0.4652967

$-0.2154489$

0.6206754

$-0.3454710$

1.0474076

1.2896539

1.8244699

1.7175101

2.2529169

2.5332221

0.5373602

$-2.3012993$

$-1.9869078$

$-3.2425813$

$-2.5145364$

0.8076178

2.3926930

1.8384300

$-0.3252493$

$-1.9362839$
$-0.7675631$

$-0.9302438$

$-0.2444957$

0.6018190

0.7458852

0.0576505

0.2376663

0.2198882

0.4312389

$-0.0813546$

$-0.0954149$

0.4274583

$-1.5481707$

$-2.0615500$

$-1.5674976$

$-2.1096313$

0.6322677

1.6769328

0.6115646

0.1458843

$-0.5791552$

1.0851466

1.1379381

0.5305811

2.1002986

1.4125339

1.1521045

$-0.3640505$

$-1.5859405$

$-1.2717907$

\section{z-1}

$\mathrm{E}(\mathrm{TPSS}-\mathrm{D} 3 / \operatorname{def} 2-\mathrm{TZVP})=-580.5839034476$ (conv) Lowest Freq. = $31.39 \mathrm{~cm}^{\wedge}-1$

30

Z-1 $(005 \mathrm{c} 3 / \mathrm{opt})$

C $\quad 1.8223596$

C $\quad 1.3547367$

$\begin{array}{ll}\text { C } & 1.3547367 \\ \text { C } & 0.1616118\end{array}$

C $\quad-0.5627217$

C $\quad-0.1053340$

1.0882635

1.6359393

2.8301306

1.9177058

$-0.1997401$

$-1.4828582$

$-0.6627305$

2.7533688

0.7358523

$-0.3884374$

1.2714749

1.3573022

2.0825434

0.0751703

3.3904811

3.6774516

2. 6517171

1.3378292

1.0429301

$-0.3462516$

$-0.5381777$

4.1908865

4.7008354

2.8764161

0.5330206

1.8361986

$-1.5178523$

$-1.7498750$

$-2.4961723$

$-2.0300703$

0.0160119

$-0.6547546$

$-1.2635992$

$-1.1881366$

$-0.5150122$

$-0.4601575$

$-0.2385906$

0.4878231

$-0.7052881$

$-1.7955172$

$-1.6572484$

0.5763803

$-0.7314552$

$-0.0329616$

$-1.7474263$

$-2.7371765$

$-3.3699258$

$-1.8277314$ 


$\begin{array}{lrrr}\mathrm{H} & 2.2762183 & -2.8209121 & -1.4572388 \\ \mathrm{C} & -0.8367526 & -1.7638870 & 2.4119393 \\ \mathrm{C} & -0.9652232 & -0.9532886 & 1.1059540 \\ \mathrm{H} & -0.9269283 & -2.6769159 & -0.2504844 \\ \mathrm{H} & 0.2117735 & -1.9848034 & 2.6332622 \\ \mathrm{H} & -1.3765437 & -2.7151482 & 2.3326213 \\ \mathrm{H} & -1.2602452 & -1.2026080 & 3.2524209 \\ \mathrm{C} & -2.4370134 & -0.5979201 & 0.8347888 \\ \mathrm{H} & -3.0324255 & -1.5068502 & 0.6855672 \\ \mathrm{H} & -2.5366622 & 0.0245337 & -0.0596788 \\ \mathrm{H} & -2.8629201 & -0.0499769 & 1.6823891 \\ \mathrm{H} & -0.3992276 & -0.0242080 & 1.2281289\end{array}$

\section{Dienol}

$\mathrm{E}(\mathrm{TPSS}-\mathrm{D} 3 / \operatorname{def} 2-\mathrm{TZVP})=-580.5723514549$ (conv) Lowest Freq. = $49.14 \mathrm{~cm}^{\wedge}-1$

30

Dienol (006s000/opt)

C $\quad-2.3132890 \quad-0.7183100$

$\begin{array}{lll}\text { C } & -3.6935209 & -0.7670084\end{array}$

C $\quad-4.5161175 \quad 0.1831829$

C $\quad-3.9454697 \quad 1.1919380$

C $\quad-2.5666130 \quad 1.2447438$

C $\quad-1.7307940 \quad 0.2774176$

$\begin{array}{lrr}\text { C } & -0.2751367 & 0.3481067\end{array}$

$\begin{array}{lll}\text { C } & 0.5779194 & -0.6989640\end{array}$

C $\quad 2.0192165 \quad-0.4708715$

$\begin{array}{lll}\text { C } & 2.8549027 & 0.2153434\end{array}$

C $\quad 2.4196439 \quad 0.8250531$

$\mathrm{H} \quad 3.1362535 \quad 0.5645214$

$\mathrm{H} \quad 2.3945858 \quad 1.9224001$

$\begin{array}{lll}\mathrm{H} & 1.4240208 & 0.4819500\end{array}$

$\begin{array}{llr}\text { C } & 4.3150457 & 0.3612318\end{array}$

$\mathrm{H} \quad 4.5589102 \quad-0.1245286$

$\mathrm{H} \quad 4.9419778 \quad-0.0762524$

$\mathrm{H} \quad 4.5990907 \quad 1.4200109$

$\mathrm{H} \quad 2.4657830 \quad-0.9623347$

C $\quad 0.1174183 \quad-2.1230804$

$\mathrm{H} \quad-0.9489725 \quad-2.1863963$

$\mathrm{H} \quad 0.6741114 \quad-2.5666048$

$\mathrm{H} \quad 0.3210196 \quad-2.7503125$

O $\quad 0.1631019 \quad 1.6544874$

$\mathrm{H} \quad 1.0999178 \quad 1.6143647$

$\mathrm{H} \quad-2.1198798 \quad 2.0316467$

$\mathrm{H} \quad-4.5785218 \quad 1.9414724$

$\mathrm{H} \quad-5.5922393 \quad 0.1458000$

$\mathrm{H} \quad-4.1260809-1.5411679$

$\mathrm{H} \quad-1.6762840 \quad-1.4378394 \quad-1.1818917$

\section{2}

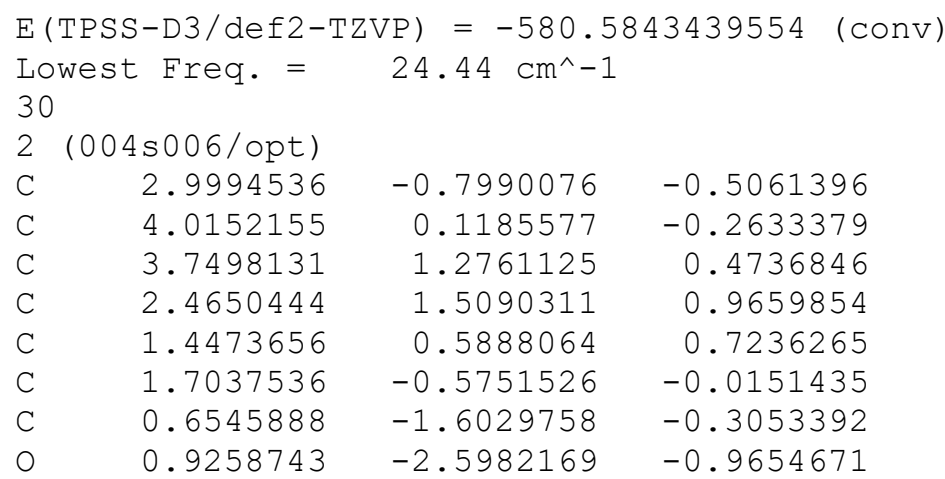




$\begin{array}{lrrr}\mathrm{C} & -0.7781468 & -1.3711265 & 0.2037458 \\ \mathrm{C} & -1.4305822 & -0.3557167 & -0.7062543 \\ \mathrm{C} & -2.0573454 & 0.7797071 & -0.3624334 \\ \mathrm{C} & -2.2217981 & 1.2737576 & 1.0500934 \\ \mathrm{H} & -1.8271952 & 0.5801033 & 1.7965615 \\ \mathrm{H} & -1.7214617 & 2.2432514 & 1.1809467 \\ \mathrm{H} & -3.2836682 & 1.4391438 & 1.2737552 \\ \mathrm{C} & -2.6633995 & 1.6695552 & -1.4174844 \\ \mathrm{H} & -3.7409634 & 1.7992842 & -1.2496300 \\ \mathrm{H} & -2.5153713 & 1.2603710 & -2.4204909 \\ \mathrm{H} & -2.2161437 & 2.6724007 & -1.3834717 \\ \mathrm{H} & -1.3874706 & -0.6145773 & -1.7652287 \\ \mathrm{H} & -0.7196237 & -0.9723034 & 1.2221112 \\ \mathrm{C} & -1.5649494 & -2.6904687 & 0.2202757 \\ \mathrm{H} & -1.6404874 & -3.1038486 & -0.7882840 \\ \mathrm{H} & -1.0648021 & -3.4330858 & 0.8498401 \\ \mathrm{H} & -2.5730273 & -2.5133887 & 0.6067863 \\ \mathrm{H} & 0.4515675 & 0.7853196 & 1.1035022 \\ \mathrm{H} & 2.2559167 & 2.4087190 & 1.5378308 \\ \mathrm{H} & 4.5426953 & 1.9944363 & 0.6631889 \\ \mathrm{H} & 5.0151822 & -0.0638911 & -0.6467380 \\ \mathrm{H} & 3.1799656 & -1.7047972 & -1.0764917\end{array}$

\section{Conformer $\mathrm{Cl}$}

$\mathrm{E}$ (TPSS-D3/def2-TZVP) $=-618.7223338601$ (conv) Lowest Freq. $=25.78 \mathrm{~cm}^{\wedge}-1$

31

C1 $(002 \mathrm{c} 1 / \mathrm{censo} / \mathrm{s} 000)$

$\begin{array}{rrrr}\text { C } & 3.5257366 & -1.3318975 & 0.1369420 \\ \mathrm{C} & 2.2867782 & -1.3656383 & -0.4858390 \\ \mathrm{C} & 1.3821962 & -0.3015686 & -0.3377898 \\ \mathrm{C} & 1.7308351 & 0.8207147 & 0.4393101 \\ \mathrm{C} & 2.9889221 & 0.8429377 & 1.0530945 \\ \mathrm{C} & 3.8772833 & -0.2190649 & 0.9092258 \\ \mathrm{H} & 4.8490344 & -0.1784916 & 1.3939777 \\ \mathrm{H} & 3.2748418 & 1.7098703 & 1.6447156 \\ \mathrm{C} & 0.7779285 & 1.9801215 & 0.6166093 \\ \mathrm{C} & -0.2821025 & 2.0472420 & -0.4870875 \\ \mathrm{H} & -1.0317420 & 2.8096159 & -0.2466027 \\ \mathrm{C} & -0.9719876 & 0.6877099 & -0.7002267 \\ \mathrm{C} & -1.7511268 & 0.2326030 & 0.5143270 \\ \mathrm{C} & -2.8305716 & -0.5659378 & 0.5164560 \\ \mathrm{C} & -3.4522994 & -1.1577136 & -0.7189393 \\ \mathrm{H} & -3.3882682 & -2.2530758 & -0.6808244 \\ \mathrm{H} & -4.5199263 & -0.9048311 & -0.7698570 \\ \mathrm{H} & -2.9610218 & -0.8372338 & -1.6384777 \\ \mathrm{C} & -3.5020968 & -0.9389634 & 1.8130803 \\ \mathrm{H} & -3.5029092 & -2.0286912 & 1.9500400 \\ \mathrm{H} & -4.5535864 & -0.6217358 & 1.8131689 \\ \mathrm{H} & -3.0024912 & -0.4830510 & 2.6726925 \\ \mathrm{H} & -1.3700772 & 0.5687181 & 1.4781327 \\ \mathrm{C} & 0.0667531 & -0.3890048 & -1.0315434 \\ \mathrm{O} & -0.1907271 & -1.2937174 & -1.8168789 \\ \mathrm{H} & -1.6353025 & 0.7416764 & -1.5679544 \\ \mathrm{H} & 0.1979937 & 2.3464445 & -1.4273814 \\ \mathrm{H} & 1.347733 & 2.9157729 & 0.6520537 \\ \mathrm{H} & 0.2907045 & 1.8894030 & 1.5974300 \\ \mathrm{H} & 1.9807143 & -2.2082740 & -1.0984340 \\ \mathrm{H} & 4.2195130 & -2.1600142 & 0.0245713\end{array}$




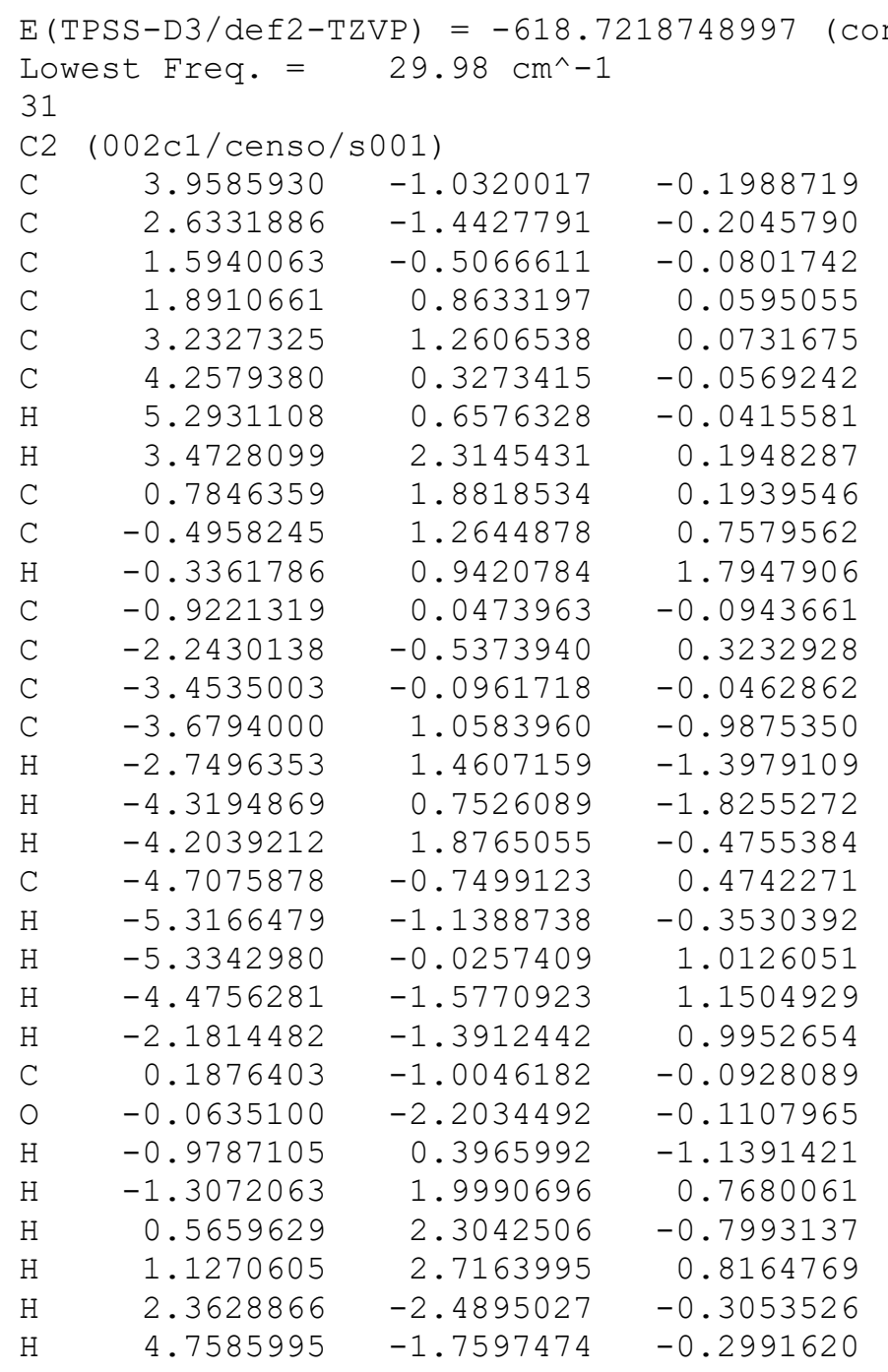

\section{Conformer C3}

$\mathrm{E}(\mathrm{TPSS}-\mathrm{D} 3 / \operatorname{def} 2-\mathrm{TZVP})=-618.7213840157$ (conv) Lowest Freq. $=23.83 \mathrm{~cm}^{\wedge}-1$

31

C3 (002c1/censo/s003)

$\begin{array}{lrrr}\mathrm{C} & 3.6031354 & -1.5447095 & 0.2028772 \\ \mathrm{C} & 2.2187632 & -1.6316262 & 0.2405495 \\ \mathrm{C} & 1.4250660 & -0.4861607 & 0.0663697 \\ \mathrm{C} & 2.0326962 & 0.7664836 & -0.1480575 \\ \mathrm{C} & 3.4297348 & 0.8384841 & -0.1802183 \\ \mathrm{C} & 4.2101226 & -0.3018507 & -0.0078868 \\ \mathrm{H} & 5.2936723 & -0.2227015 & -0.0318521 \\ \mathrm{H} & 3.9077176 & 1.8032360 & -0.3347086 \\ \mathrm{C} & 1.1904306 & 2.0036007 & -0.3409766 \\ \mathrm{C} & -0.1588150 & 1.8806338 & 0.3666228 \\ \mathrm{H} & -0.0028770 & 1.8215506 & 1.4522034 \\ \mathrm{C} & -0.9111825 & 0.6245345 & -0.1043950 \\ \mathrm{C} & -2.2716906 & 0.5119659 & 0.5235734 \\ \mathrm{C} & -3.3607931 & -0.0577476 & -0.0086063 \\ \mathrm{C} & -3.3957347 & -0.7016269 & -1.3679681 \\ \mathrm{H} & -2.4755086 & -0.5455344 & -1.9353463 \\ \mathrm{H} & -3.5372297 & -1.7849090 & -1.2648433 \\ \mathrm{H} & -4.2402826 & -0.3203574 & -1.9570265 \\ \mathrm{C} & -4.6541603 & -0.1192047 & 0.7616141 \\ \mathrm{H} & -4.9640011 & -1.1617266 & 0.9140427 \\ \mathrm{H} & -5.4649883 & 0.3731652 & 0.2077665\end{array}$




$\begin{array}{rrrr}\mathrm{H} & -4.5622719 & 0.3594078 & 1.7407831 \\ \mathrm{H} & -2.3452742 & 0.9184640 & 1.5334765 \\ \mathrm{C} & -0.0588095 & -0.6433517 & 0.1072691 \\ \mathrm{O} & -0.5766125 & -1.7404918 & 0.2625989 \\ \mathrm{H} & -1.0132714 & 0.6932743 & -1.2004490 \\ \mathrm{H} & -0.7750164 & 2.7658381 & 0.1755172 \\ \mathrm{H} & 1.0172613 & 2.1573599 & -1.4173919 \\ \mathrm{H} & 1.7418273 & 2.8821245 & 0.0125309 \\ \mathrm{H} & 1.7130057 & -2.5785520 & 0.4027061 \\ \mathrm{H} & 4.2120705 & -2.4338481 & 0.3383649\end{array}$

\section{Enol-12-cat12 si}

\begin{tabular}{|c|c|c|c|}
\hline \multicolumn{4}{|c|}{$\begin{array}{l}\text { E(TPSS-D3/def2-TZVP) }=-1655 . \\
\text { Lowest Freq. }=17.08 \mathrm{~cm}^{\wedge}-1 \\
79\end{array}$} \\
\hline & $-12-\operatorname{cat} 12$ si & (011c1/opt) & \\
\hline $\mathrm{C}$ & $-3.90420 \overline{8} 0$ & 1.2017319 & -1.6203833 \\
\hline $\mathrm{C}$ & -4.7390279 & 1.8532336 & -0.7126397 \\
\hline $\mathrm{C}$ & -4.1829488 & 2.6965673 & 0.2529213 \\
\hline $\mathrm{C}$ & -2.8046962 & 2.8843595 & 0.3346619 \\
\hline $\mathrm{C}$ & -1.9548169 & 2.2137884 & -0.5745267 \\
\hline $\mathrm{C}$ & -2.5247558 & 1.3891373 & -1.5565903 \\
\hline $\mathrm{C}$ & -0.4984739 & 2.3965273 & -0.4771774 \\
\hline $\mathrm{C}$ & 0.0720813 & 2.9454095 & 0.6345915 \\
\hline $\mathrm{C}$ & -0.8232465 & 3.3171479 & 1.8049726 \\
\hline $\mathrm{C}$ & -2.1803985 & 3.8328363 & 1.3257835 \\
\hline $\mathrm{H}$ & -4.3266268 & 0.5536291 & -2.3834108 \\
\hline $\mathrm{H}$ & -5.8152915 & 1.7131234 & -0.7581875 \\
\hline $\mathrm{H}$ & -4.8304832 & 3.2174462 & 0.9548770 \\
\hline $\mathrm{H}$ & -1.8790527 & 0.9032006 & -2.2798573 \\
\hline $\mathrm{H}$ & -0.3336346 & 4.0801953 & 2.4190050 \\
\hline $\mathrm{H}$ & -2.8562007 & 4.0012713 & 2.1711730 \\
\hline $\mathrm{H}$ & -0.9639196 & 2.4346243 & 2.4505349 \\
\hline $\mathrm{H}$ & -2.0314491 & 4.8048723 & 0.8305167 \\
\hline 0 & 0.2724460 & 1.9475212 & -1.5128468 \\
\hline $\mathrm{C}$ & 1.5106027 & 3.0529850 & 0.8568681 \\
\hline $\mathrm{C}$ & 2.4682526 & 3.5165802 & 0.0191935 \\
\hline $\mathrm{H}$ & 1.8310925 & 2.7791101 & 1.8664799 \\
\hline $\mathrm{C}$ & 3.9120295 & 3.5398674 & 0.4525397 \\
\hline $\mathrm{H}$ & 4.0440912 & 3.1055313 & 1.4476021 \\
\hline $\mathrm{H}$ & 4.2964785 & 4.5686000 & 0.4638827 \\
\hline $\mathrm{H}$ & 4.5422321 & 2.9808837 & -0.2528240 \\
\hline C & 2.1809435 & 4.0926217 & -1.3379715 \\
\hline $\mathrm{H}$ & 1.1230210 & 4.3331357 & -1.4575206 \\
\hline $\mathrm{H}$ & 2.4464965 & 3.3850650 & -2.1331076 \\
\hline $\mathrm{H}$ & 2.7800831 & 4.9996069 & -1.4905302 \\
\hline $\mathrm{H}$ & 0.1504590 & 0.9434259 & -1.6716691 \\
\hline $\mathrm{C}$ & 1.5197148 & -3.0027873 & -2.4279754 \\
\hline $\mathrm{C}$ & -0.0080643 & -3.0545144 & -2.7033744 \\
\hline $\mathrm{C}$ & -0.5440285 & -1.5850352 & -2.5674434 \\
\hline $\mathrm{N}$ & 0.4660938 & -0.6952141 & -1.9512978 \\
\hline $\mathrm{C}$ & 0.8883705 & -1.3531493 & -0.6752557 \\
\hline $\mathrm{C}$ & 1.7991500 & -2.5594474 & -0.9800109 \\
\hline $\mathrm{C}$ & 2.1208350 & -1.9442624 & -3.3654608 \\
\hline $\mathrm{C}$ & 1.6105549 & -0.5568889 & -2.9002729 \\
\hline $\mathrm{C}$ & -0.7395070 & -4.0106742 & -1.8073430 \\
\hline $\mathrm{C}$ & -1.3977743 & -5.0911069 & -2.2298441 \\
\hline $\mathrm{C}$ & 1.4583605 & -0.3544845 & 0.3493839 \\
\hline $\mathrm{C}$ & 1.6507627 & -1.0427576 & 1.6856474 \\
\hline 0 & 2.6852403 & 0.2134924 & -0.1235644 \\
\hline $\mathrm{C}$ & 0.7985744 & -2.1052933 & 3.7113951 \\
\hline
\end{tabular}




$\begin{array}{lrrr}\mathrm{N} & 2.0562599 & -2.4172712 & 4.1363879 \\ \mathrm{C} & 3.0648811 & -2.0400629 & 3.3690027 \\ \mathrm{C} & 2.9128763 & -1.3567012 & 2.1443480 \\ \mathrm{C} & 0.5300499 & -1.4066561 & 2.4893873 \\ \mathrm{C} & -0.8160065 & -1.1102074 & 2.1398552 \\ \mathrm{C} & -1.8526053 & -1.5229666 & 2.9577595 \\ \mathrm{C} & -1.5861482 & -2.2204687 & 4.1655157 \\ \mathrm{C} & -0.2944758 & -2.4963333 & 4.5304362 \\ \mathrm{O} & -3.1751471 & -1.3138418 & 2.6973818 \\ \mathrm{C} & -3.5020528 & -0.7096835 & 1.4379378 \\ \mathrm{H} & 1.9559481 & -3.9872127 & -2.6212158 \\ \mathrm{H} & -0.1483222 & -3.3860713 & -3.7396324 \\ \mathrm{H} & -1.4471058 & -1.5593503 & -1.9495609 \\ \mathrm{H} & -0.7959870 & -1.1751621 & -3.5509842 \\ \mathrm{H} & -0.0428510 & -1.7238487 & -0.2352596 \\ \mathrm{H} & 1.6094215 & -3.3632993 & -0.2629903 \\ \mathrm{H} & 2.8533784 & -2.2758948 & -0.8829795 \\ \mathrm{H} & 1.8263829 & -2.1500939 & -4.4015875 \\ \mathrm{H} & 3.2147117 & -1.9777978 & -3.3215271 \\ \mathrm{H} & 1.2567907 & 0.0485766 & -3.7404557 \\ \mathrm{H} & 2.3876807 & -0.0018773 & -2.3775105 \\ \mathrm{H} & -0.7260026 & -3.7870274 & -0.7394695 \\ \mathrm{H} & -1.4393903 & -5.3524060 & -3.2855192 \\ \mathrm{H} & -1.9152448 & -5.7515980 & -1.5402437 \\ \mathrm{H} & 0.7247042 & 0.4496727 & 0.4744380 \\ \mathrm{H} & 2.5478432 & 1.1819820 & -0.1146528 \\ \mathrm{H} & 4.0625250 & -2.2909756 & 3.7280160 \\ \mathrm{H} & 3.7802102 & -1.0688606 & 1.5599641 \\ \mathrm{H} & -1.0275350 & -0.5442323 & 1.2407076 \\ \mathrm{H} & -2.4290686 & -2.5214154 & 4.7800820 \\ \mathrm{H} & -0.0668965 & -3.0239846 & 5.4514546 \\ \mathrm{H} & -4.5900492 & -0.6685832 & 1.4065761 \\ \mathrm{H} & -3.0925514 & 0.3027039 & 1.3645110 \\ \mathrm{H} & -3.1255852 & -1.3209640 & 0.6088827 \\ & & & \\ & & & \end{array}$

\section{Enol-12-cat12}

$\begin{array}{lccc}\text { E (TPSS-D3/def2-TZVP) }=-1655.859258882 & \text { (conv) } \\ \text { Lowest Freq. }= & 8.61 \mathrm{Cm}^{\wedge}-1 \\ \text { 79 } & & \\ \text { Enol-12-cat12_re } & (012 \mathrm{C} 2 / \text { opt) } \\ \mathrm{C} & -1.1494238 & 2.0050403 & -4.8116043 \\ \mathrm{C} & -0.0188585 & 2.6595921 & -5.3021781 \\ \mathrm{C} & 0.9328061 & 3.1619621 & -4.4105823 \\ \mathrm{C} & 0.7722668 & 3.0158806 & -3.0342546 \\ \mathrm{C} & -0.3655038 & 2.3431913 & -2.5374213 \\ \mathrm{C} & -1.3202659 & 1.8467175 & -3.4381849 \\ \mathrm{C} & -0.5164478 & 2.1635836 & -1.0894331 \\ \mathrm{C} & 0.1901461 & 2.9117044 & -0.1967731 \\ \mathrm{C} & 1.0999169 & 3.9918720 & -0.7572620 \\ \mathrm{C} & 1.7892854 & 3.5152540 & -2.0378126 \\ \mathrm{H} & -1.8998812 & 1.6235072 & -5.4986653 \\ \mathrm{H} & 0.1194422 & 2.7863156 & -6.3722070 \\ \mathrm{H} & 1.8126986 & 3.6764689 & -4.7910497 \\ \mathrm{H} & -2.2008786 & 1.3482432 & -3.0443561 \\ \mathrm{H} & 1.8388532 & 4.2960766 & -0.0128436 \\ \mathrm{H} & 2.3978583 & 4.3113642 & -2.4804103 \\ \mathrm{H} & 0.4977411 & 4.8880208 & -0.9764434 \\ \mathrm{H} & 2.4721564 & 2.6868739 & -1.7847589 \\ \mathrm{O} & -1.4298656 & 1.2314454 & -0.6618289 \\ \mathrm{C} & -0.0487702 & 2.7869854 & 1.2433372 \\ \mathrm{C} & 0.8644045 & 2.7632464 & 2.2367304 \\ \mathrm{H} & -1.0921443 & 2.6722886 & 1.5370865 \\ & & & \end{array}$




\begin{tabular}{|c|c|c|c|}
\hline C & 0.4343082 & 2.6900629 & 3.6764403 \\
\hline $\mathrm{H}$ & -0.6542202 & 2.6597838 & 3.7679526 \\
\hline $\mathrm{H}$ & 0.8054741 & 3.5592161 & 4.2375637 \\
\hline $\mathrm{H}$ & 0.8474061 & 1.7985347 & 4.1652007 \\
\hline C & 2.3534556 & 2.7298977 & 2.0134008 \\
\hline $\mathrm{H}$ & 2.6122554 & 2.5847567 & 0.9603829 \\
\hline $\mathrm{H}$ & 2.7935119 & 1.9097185 & 2.5953144 \\
\hline $\mathrm{H}$ & 2.8392242 & 3.6537154 & 2.3560636 \\
\hline $\mathrm{H}$ & -1.1528691 & 0.3231204 & -1.0617888 \\
\hline C & 0.5443088 & -3.4786837 & -1.9057420 \\
\hline C & -1.0033137 & -3.5692458 & -1.9789437 \\
\hline C & -1.5517009 & -2.1123016 & -1.7984745 \\
\hline $\mathrm{N}$ & -0.4764729 & -1.1543723 & -1.456691 \\
\hline C & 0.2006511 & -1.6633056 & -0.2237940 \\
\hline C & 0.9952842 & -2.9557113 & -0.5321264 \\
\hline C & 1.0129104 & -2.4777906 & -2.9741012 \\
\hline C & 0.4689483 & -1.0715217 & -2.6046664 \\
\hline C & -1.5865897 & -4.5134679 & -0.9702543 \\
\hline C & -2.2413285 & -5.6363953 & -1.26908 \\
\hline C & 1.0263792 & -0.5748562 & 0.48631 \\
\hline C & 1.2265403 & -0.9389318 & 1.9437266 \\
\hline O & 2.2774794 & -0.4058921 & -0.2 \\
\hline $\mathrm{C}$ & 0.4023817 & -1.1787897 & 4.2293 \\
\hline $\mathrm{N}$ & 1.6075053 & -1.6456711 & 4.6667 \\
\hline$C$ & 2.5773224 & -1.7486264 & $3.7^{7}$ \\
\hline $\mathrm{C}$ & 2.4355363 & -1.4101276 & 2.4 \\
\hline C & 0.1497500 & -0.8058415 & 2.869654 \\
\hline C & -1.1308621 & -0.3127234 & 2.504817 \\
\hline C & -2.1243158 & -0.2035040 & 3.4598986 \\
\hline C & -1.8785789 & -0.5757437 & 4.8081667 \\
\hline C & -0.6462916 & -1.0478167 & $5.1^{\circ}$ \\
\hline O & -3.3824136 & 0.2651553 & $3.2138^{\prime}$ \\
\hline C & -3.7026084 & 0.5737686 & 1.848991 \\
\hline $\mathrm{H}$ & 0.9713765 & -4.4685962 & -2.0931349 \\
\hline $\mathrm{H}$ & -1.2686628 & -3.9305619 & -2.980112 \\
\hline $\mathrm{H}$ & -2.2994801 & -2.0787463 & -0.998946 \\
\hline $\mathrm{H}$ & -2.0269808 & -1.7598714 & -2.7190 \\
\hline $\mathrm{H}$ & -0.6214916 & -1.8994664 & 0.4596 \\
\hline $\mathrm{H}$ & 0.8307007 & -3.6968062 & 0.255236 \\
\hline $\mathrm{H}$ & 2.0677232 & -2.7415403 & -0.5644472 \\
\hline $\mathrm{H}$ & 0.6527116 & -2.7897453 & -3.961537 \\
\hline $\mathrm{H}$ & 2.1072216 & -2.4619787 & -3.012261 \\
\hline $\mathrm{H}$ & -0.0757114 & -0.6160064 & -3.435040 \\
\hline $\mathrm{H}$ & 1.2729575 & -0.3966149 & -2.315497 \\
\hline $\mathrm{H}$ & -1.4628650 & -4.2373633 & 0.078396 \\
\hline $\mathrm{H}$ & -2.3930494 & -5.9458105 & -2.301357 \\
\hline $\mathrm{H}$ & -2.6478523 & -6.2843726 & -0.498083 \\
\hline $\mathrm{H}$ & 0.4641177 & 0.3628407 & 0.437092 \\
\hline $\mathrm{H}$ & 2.6259444 & 0.4608352 & 0.056668 \\
\hline $\mathrm{H}$ & 3.5325258 & -2.1222876 & 4.141573 \\
\hline $\mathrm{H}$ & 3.2733231 & -1.5152588 & 1.728977 \\
\hline $\mathrm{H}$ & -1.3089603 & 0.0256611 & 1.490550 \\
\hline $\mathrm{H}$ & -2.6869556 & -0.4708949 & 5.525363 \\
\hline $\mathrm{H}$ & -0.4345365 & -1.3332545 & 6.204588 \\
\hline $\mathrm{H}$ & -4.7358871 & 0.9205850 & 1.864971 \\
\hline $\mathrm{H}$ & -3.0452121 & 1.3508680 & 1.447271 \\
\hline $\mathrm{H}$ & -3.6155603 & -0.3236579 & 1.2 \\
\hline
\end{tabular}


NMR-Spectra

${ }^{1} \mathrm{H}$ NMR (400 MHz, $\left.\mathrm{CDCl}_{3}\right): \mathbf{1}$

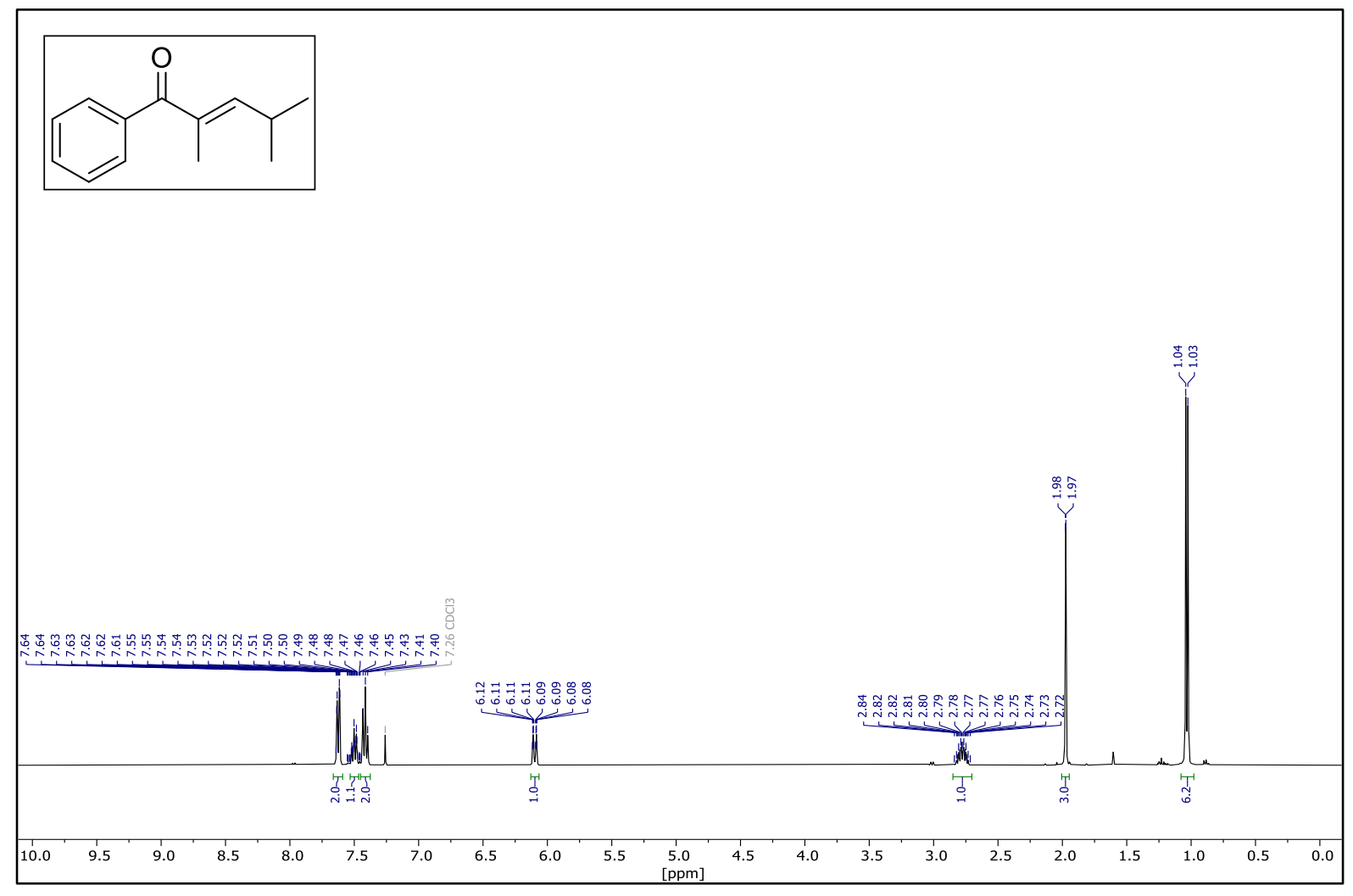

${ }^{1} \mathrm{H}$ NMR (500 MHz, $\mathrm{CDCl}_{3}$ ): 23

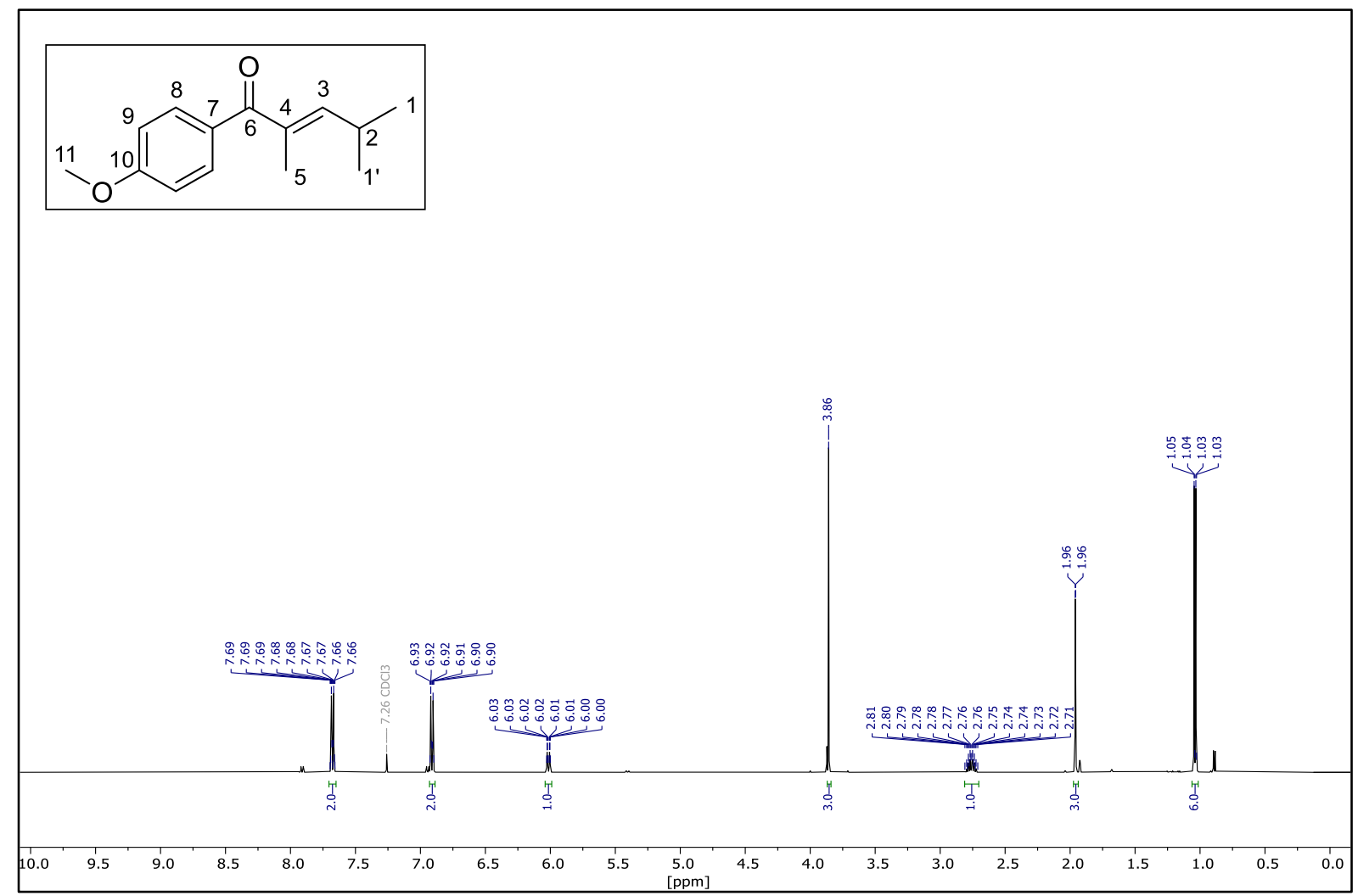


${ }^{13} \mathrm{C}$ NMR (126 MHz, $\left.\mathrm{CDCl}_{3}\right): 23$

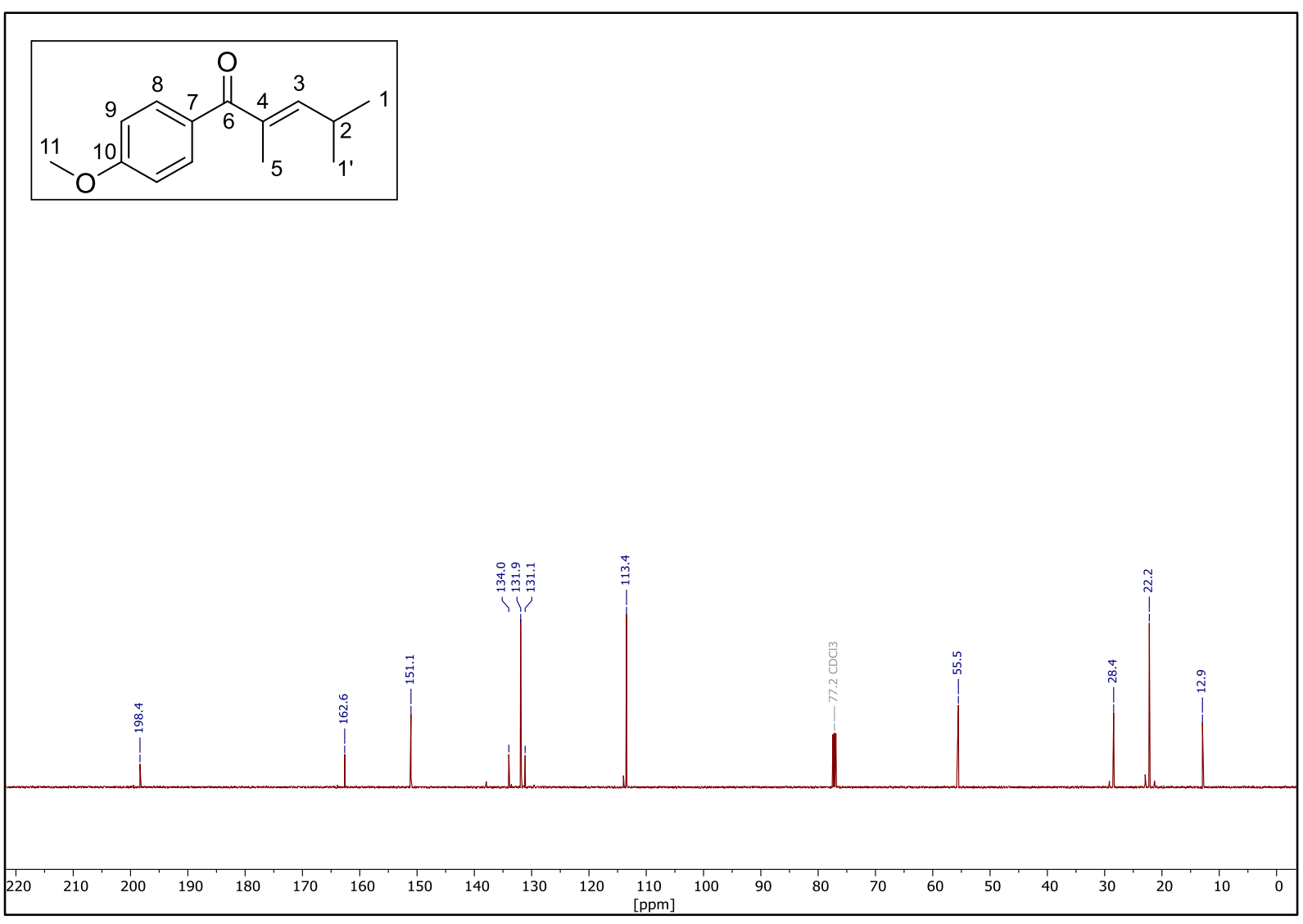

${ }^{1} \mathrm{H}$ NMR (600 MHz, $\left.\mathrm{CDCl}_{3}\right): 24$

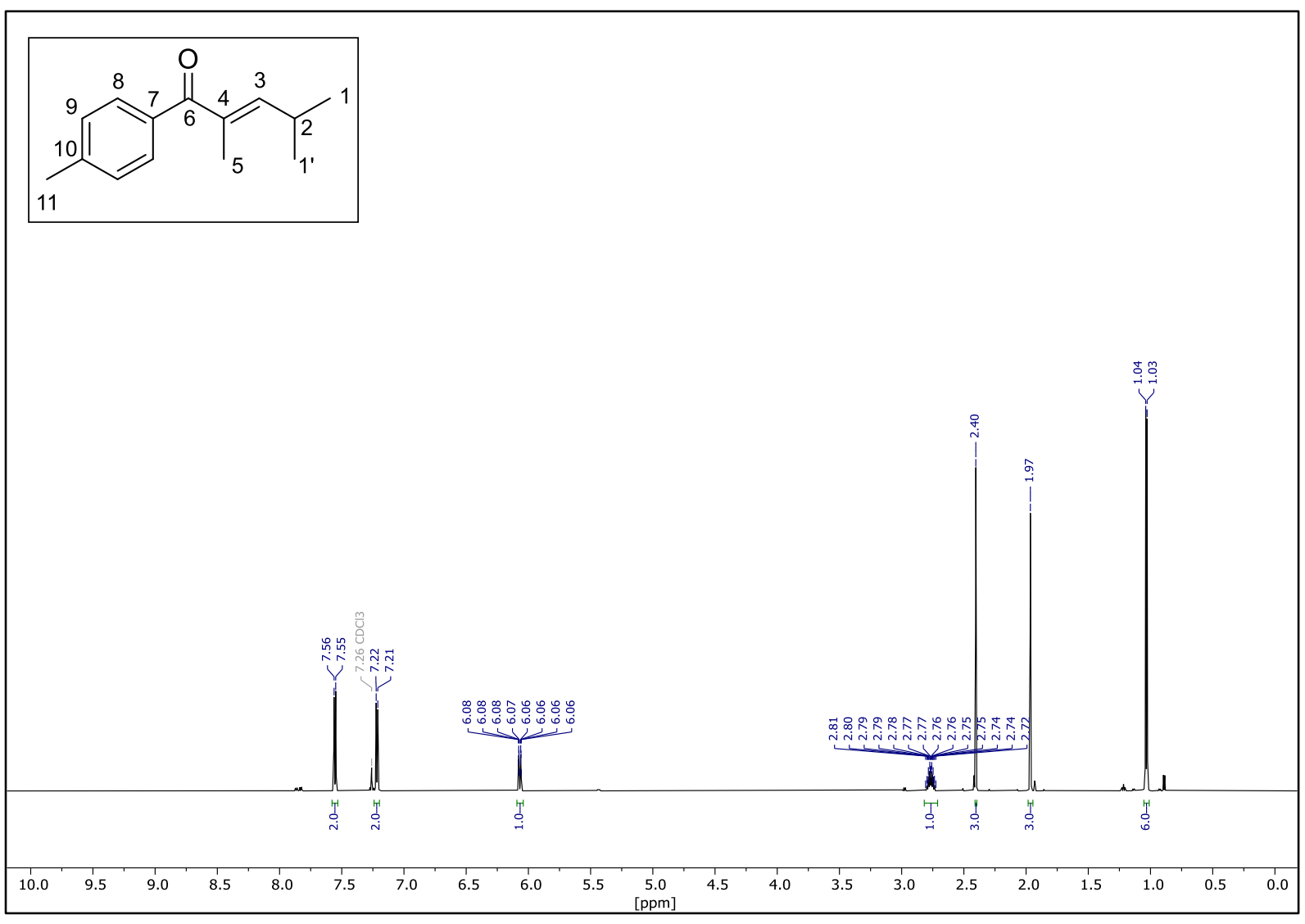


${ }^{13} \mathrm{C}$ NMR (151 MHz, $\left.\mathrm{CDCl}_{3}\right): 24$

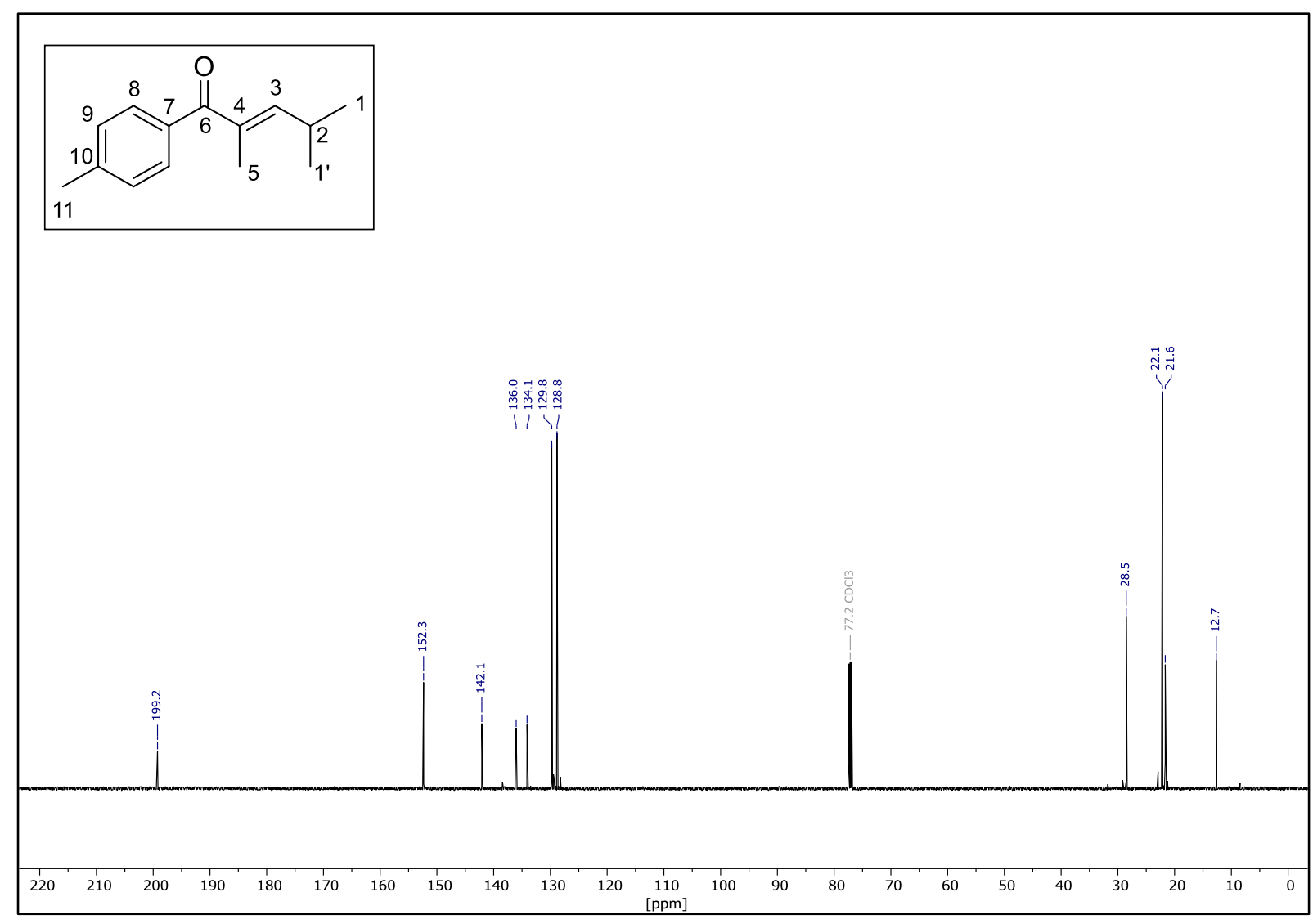

${ }^{1} \mathrm{H}$ NMR (600 MHz, $\left.\mathrm{CDCl}_{3}\right): 25$

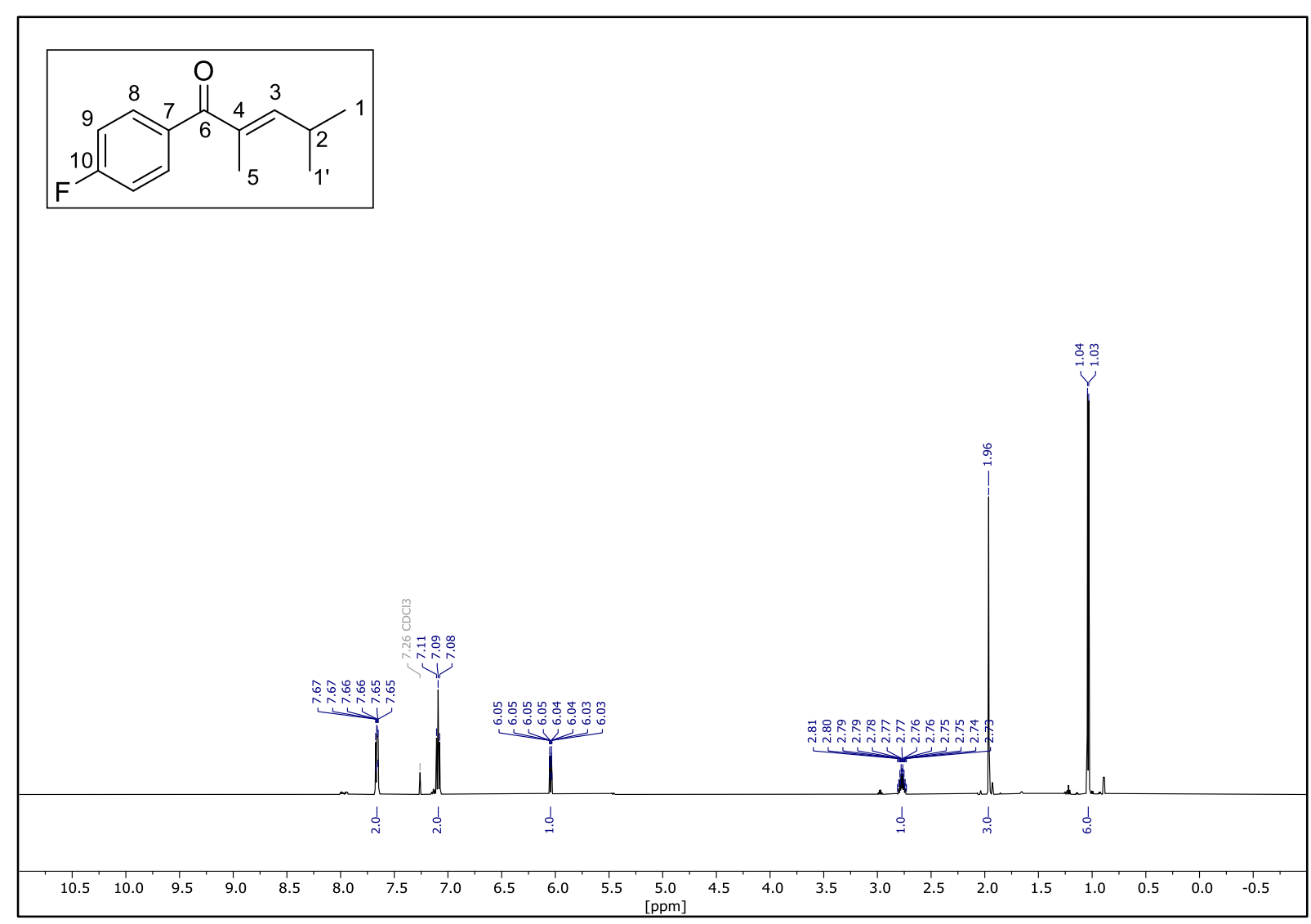


${ }^{13} \mathrm{C}$ NMR (151 MHz, $\left.\mathrm{CDCl}_{3}\right): 25$

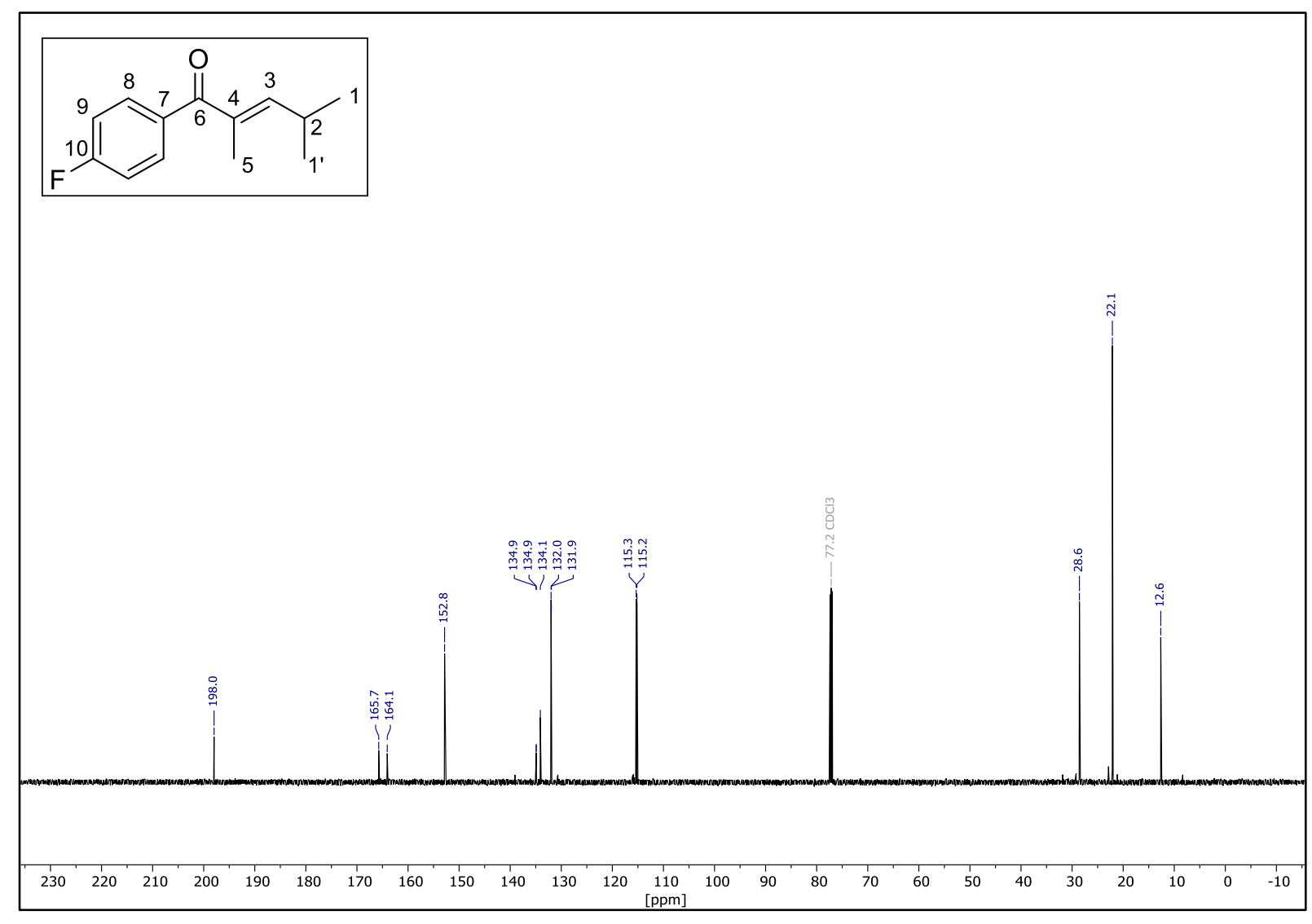

${ }^{19} \mathrm{~F}$ NMR (564 MHz, $\left.\mathrm{CDCl}_{3}\right): 25$

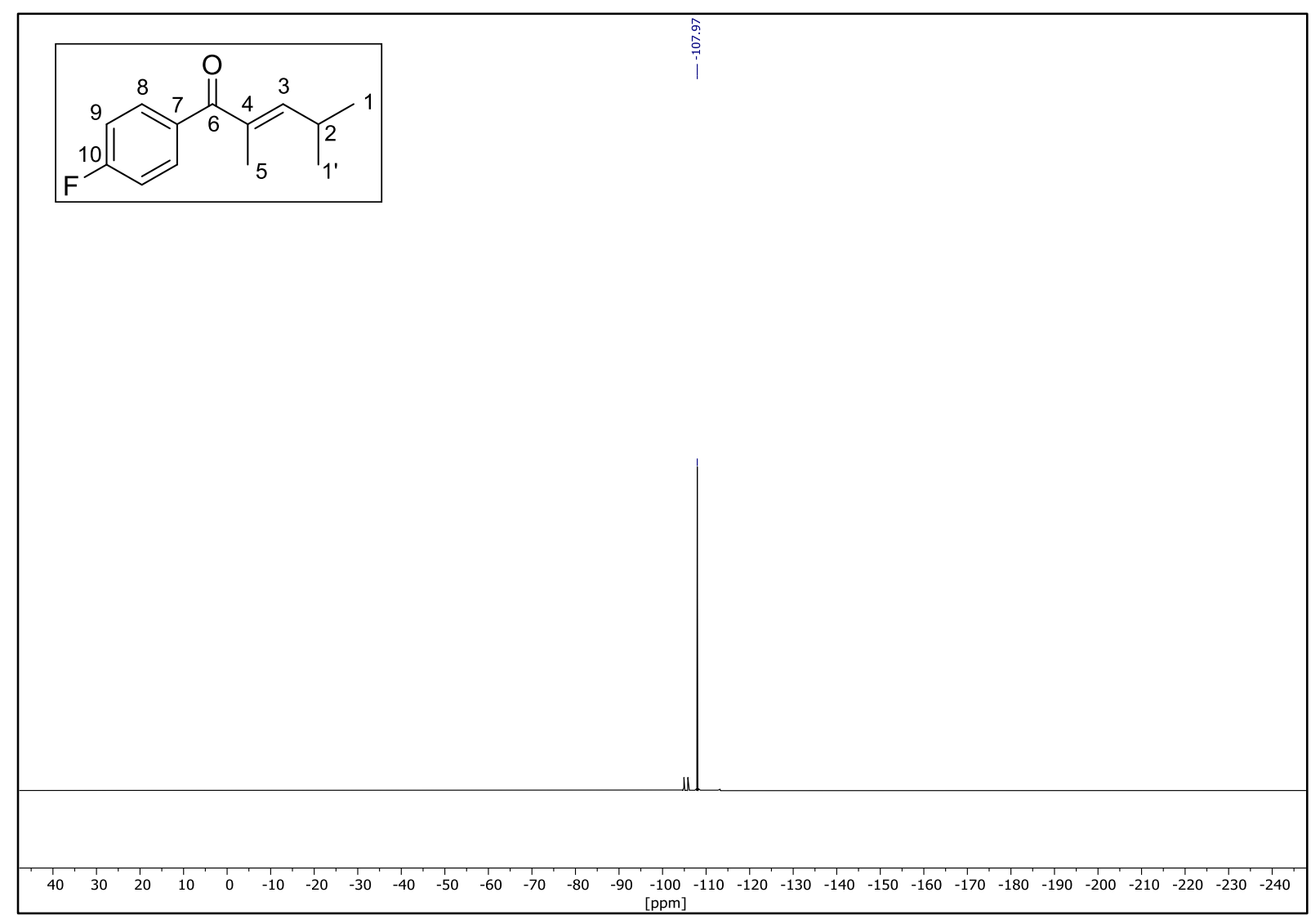


${ }^{1} \mathrm{H}$ NMR (600 MHz, $\left.\mathrm{CDCl}_{3}\right): 26$

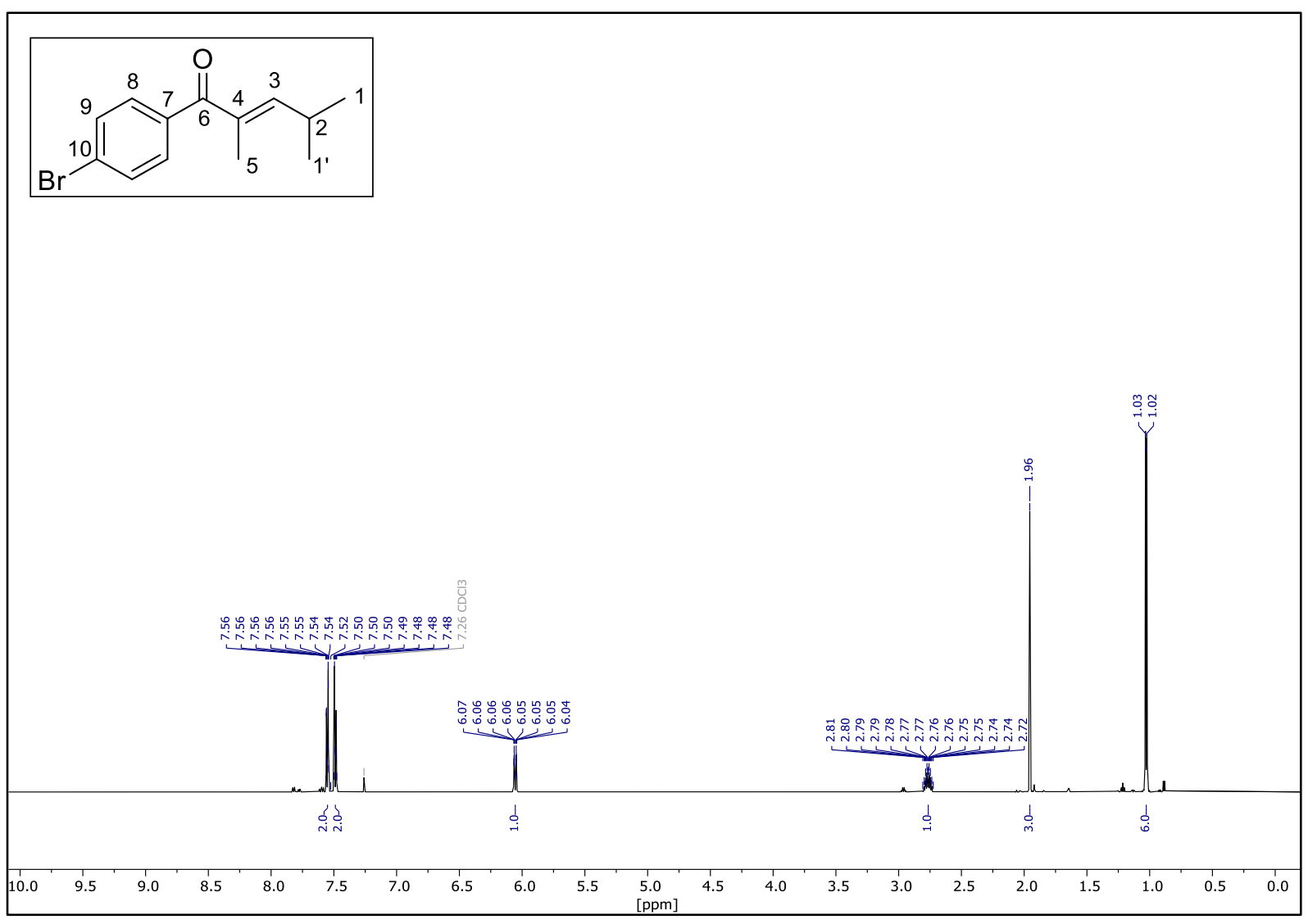

${ }^{13} \mathrm{C}$ NMR (151 MHz, $\left.\mathrm{CDCl}_{3}\right): 26$
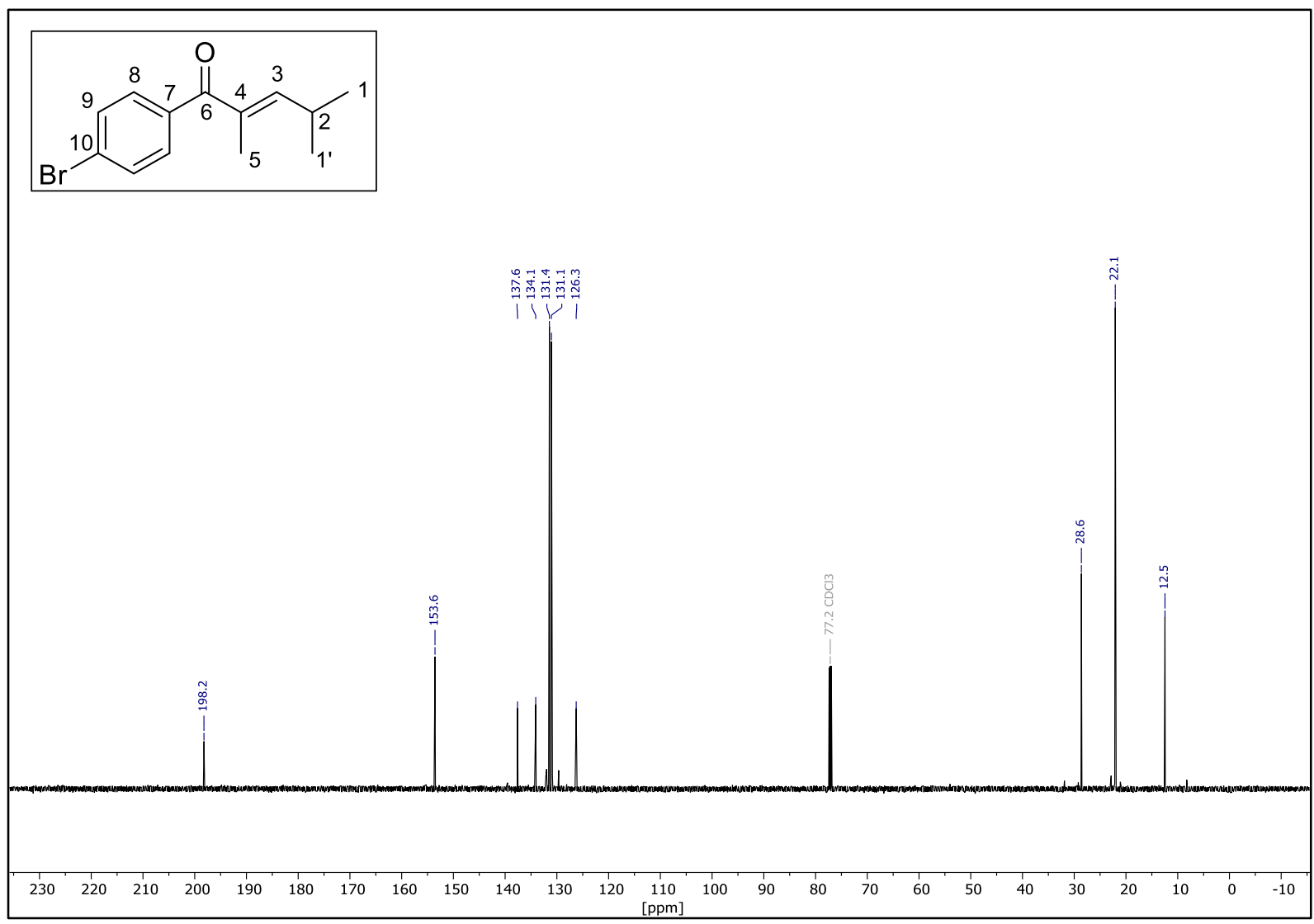
${ }^{1} \mathrm{H}$ NMR (600 MHz, $\mathrm{CDCl}_{3}$ ): 27

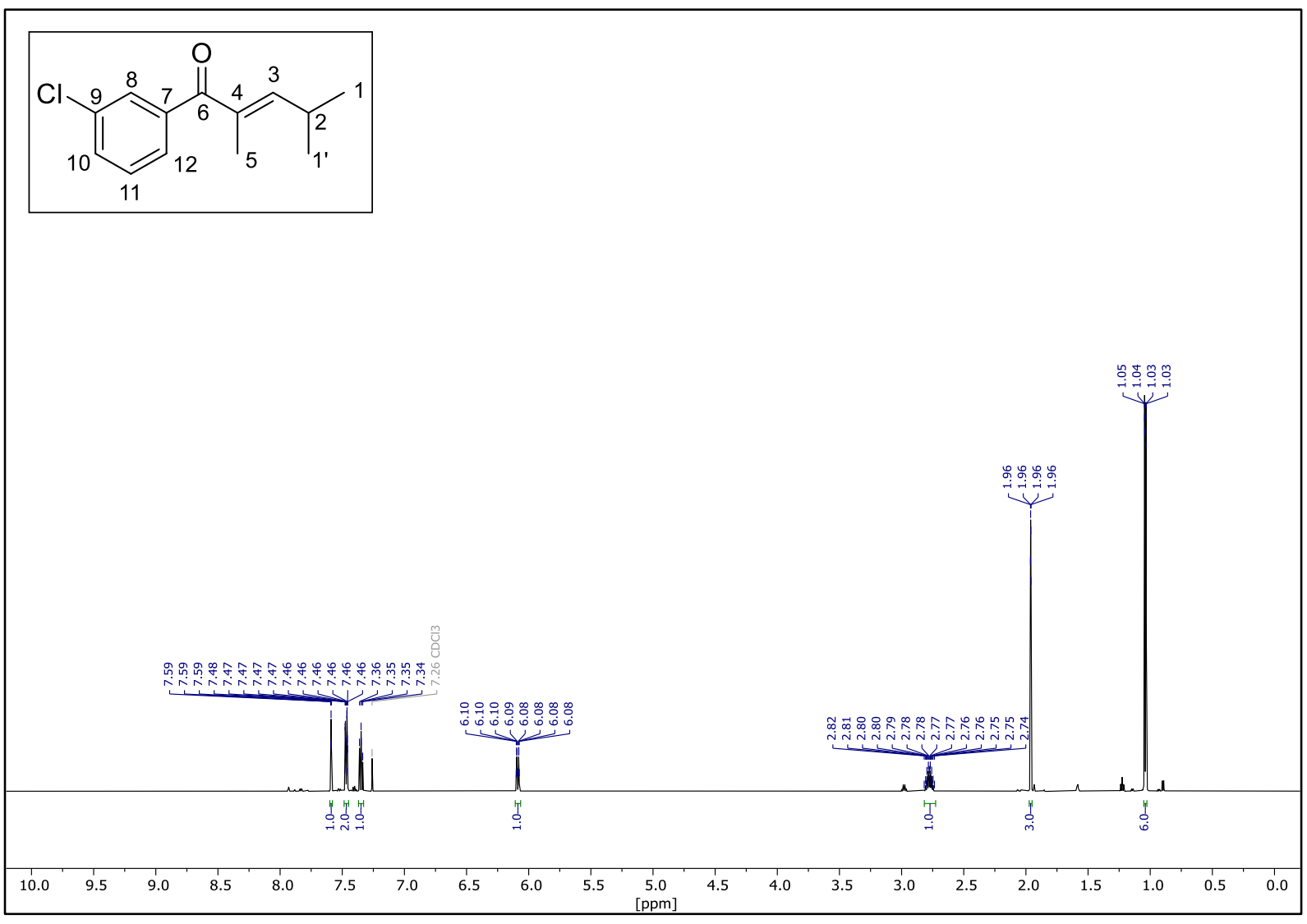

${ }^{13} \mathrm{C}$ NMR (151 MHz, $\left.\mathrm{CDCl}_{3}\right): 27$
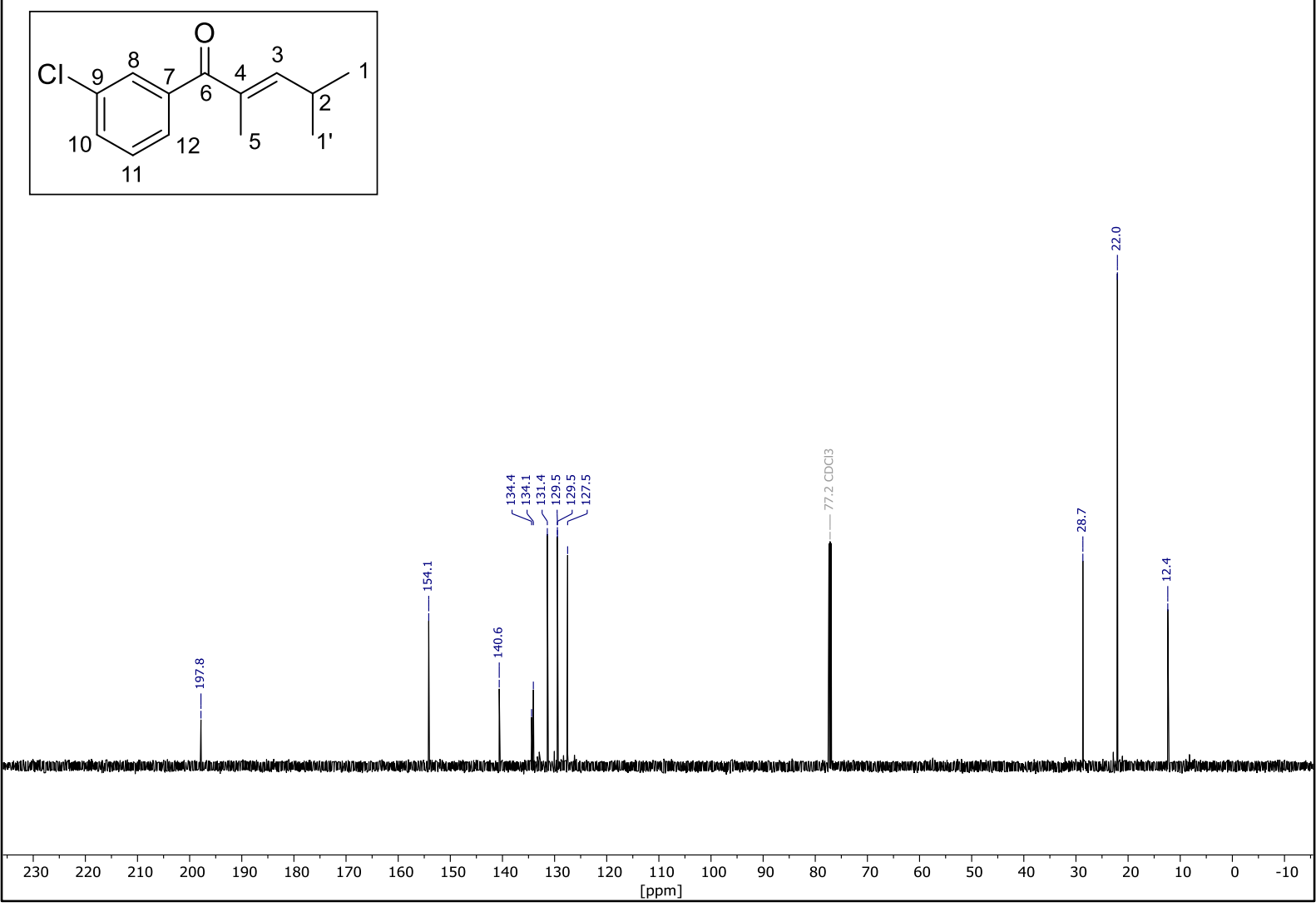
${ }^{1} \mathrm{H}$ NMR (400 MHz, $\mathrm{CDCl}_{3}$ ): 28

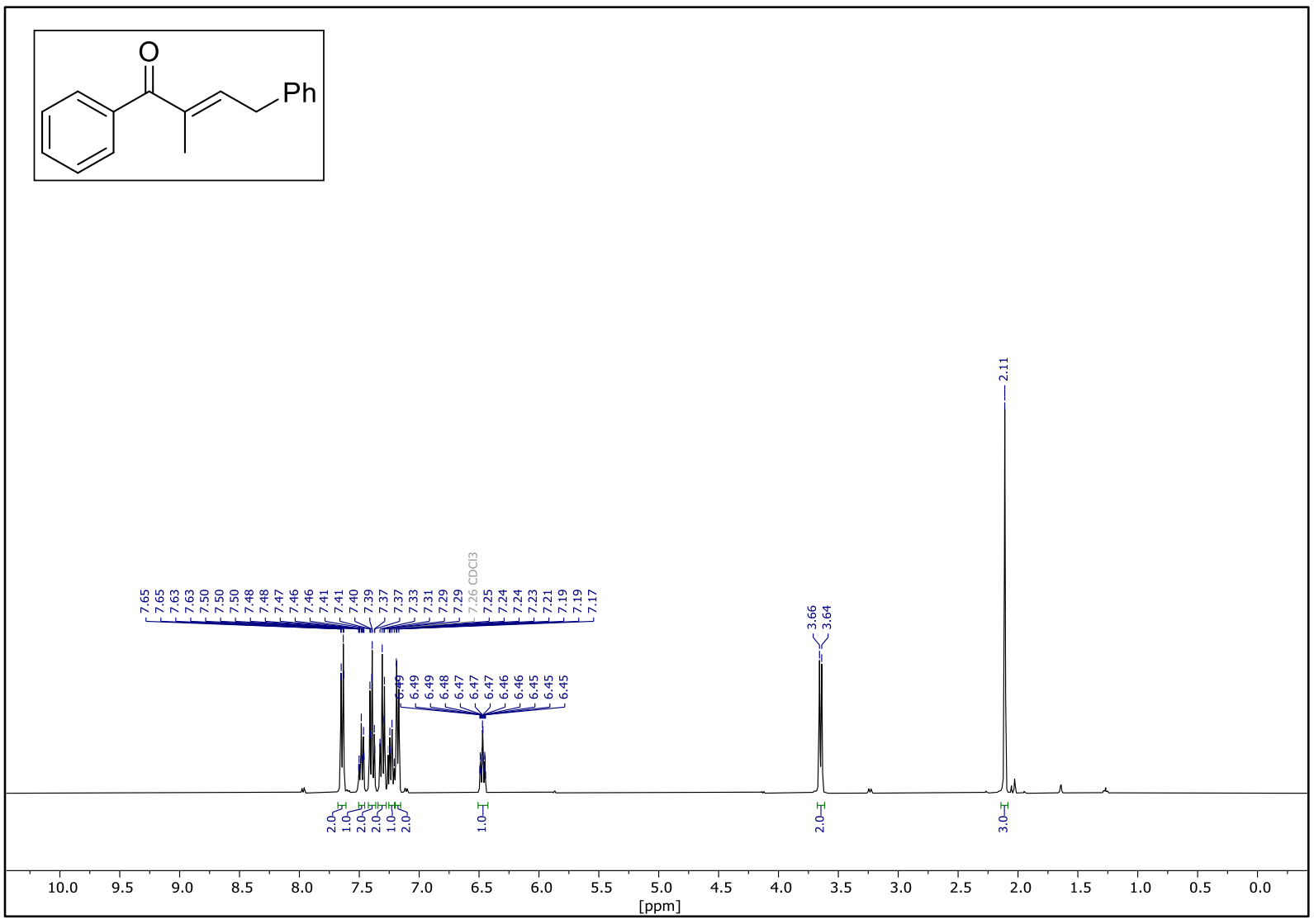

${ }^{1} \mathrm{H}$ NMR (400 MHz, $\mathrm{CDCl}_{3}$ ): 22

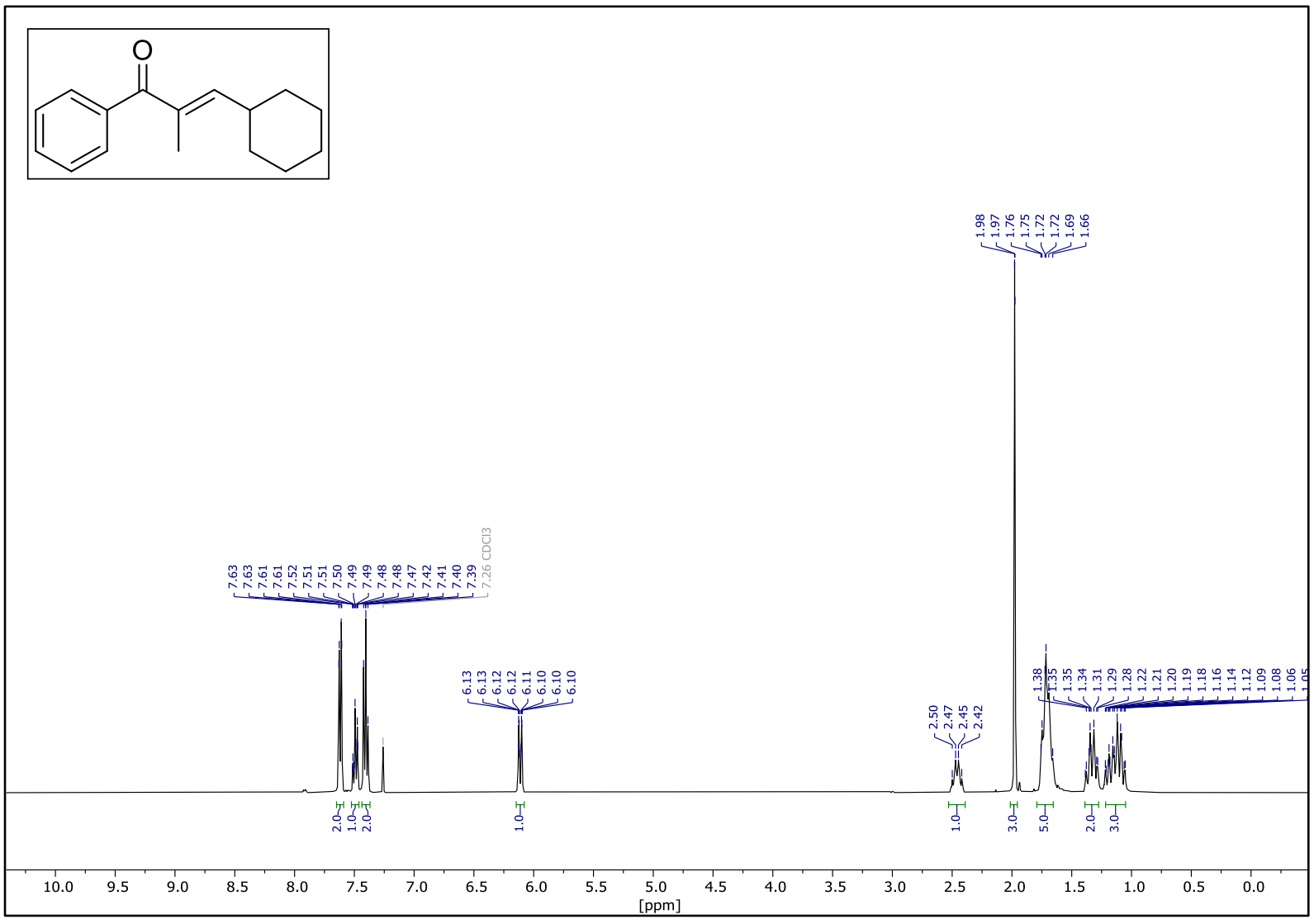


${ }^{1} \mathrm{H}$ NMR (600 MHz, $\left.\mathrm{CDCl}_{3}\right): 29$

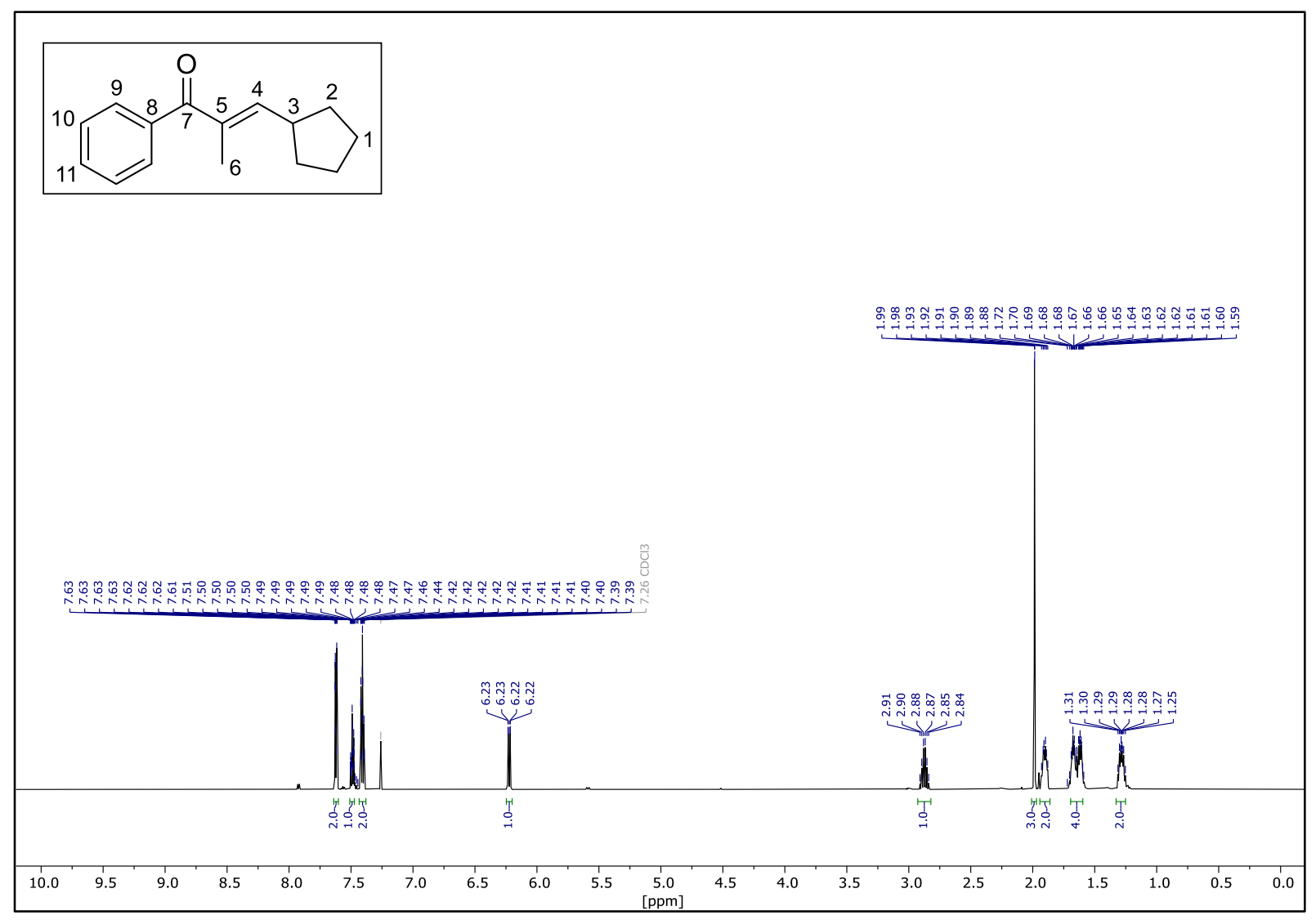

${ }^{13} \mathrm{C}$ NMR (151 MHz, $\left.\mathrm{CDCl}_{3}\right): 29$

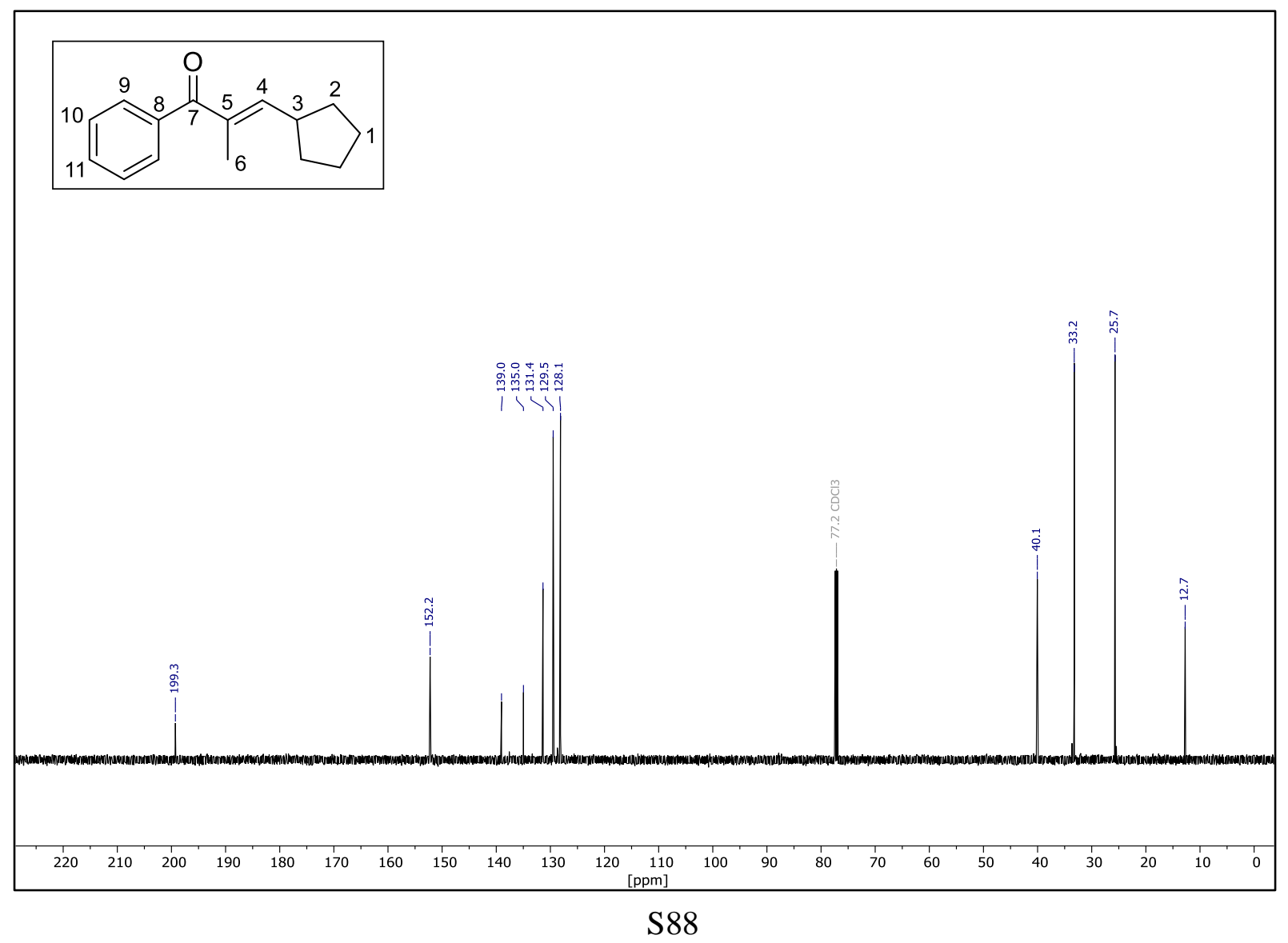


${ }^{1} \mathrm{H}$ NMR (600 MHz, $\left.\mathrm{CDCl}_{3}\right): 30$

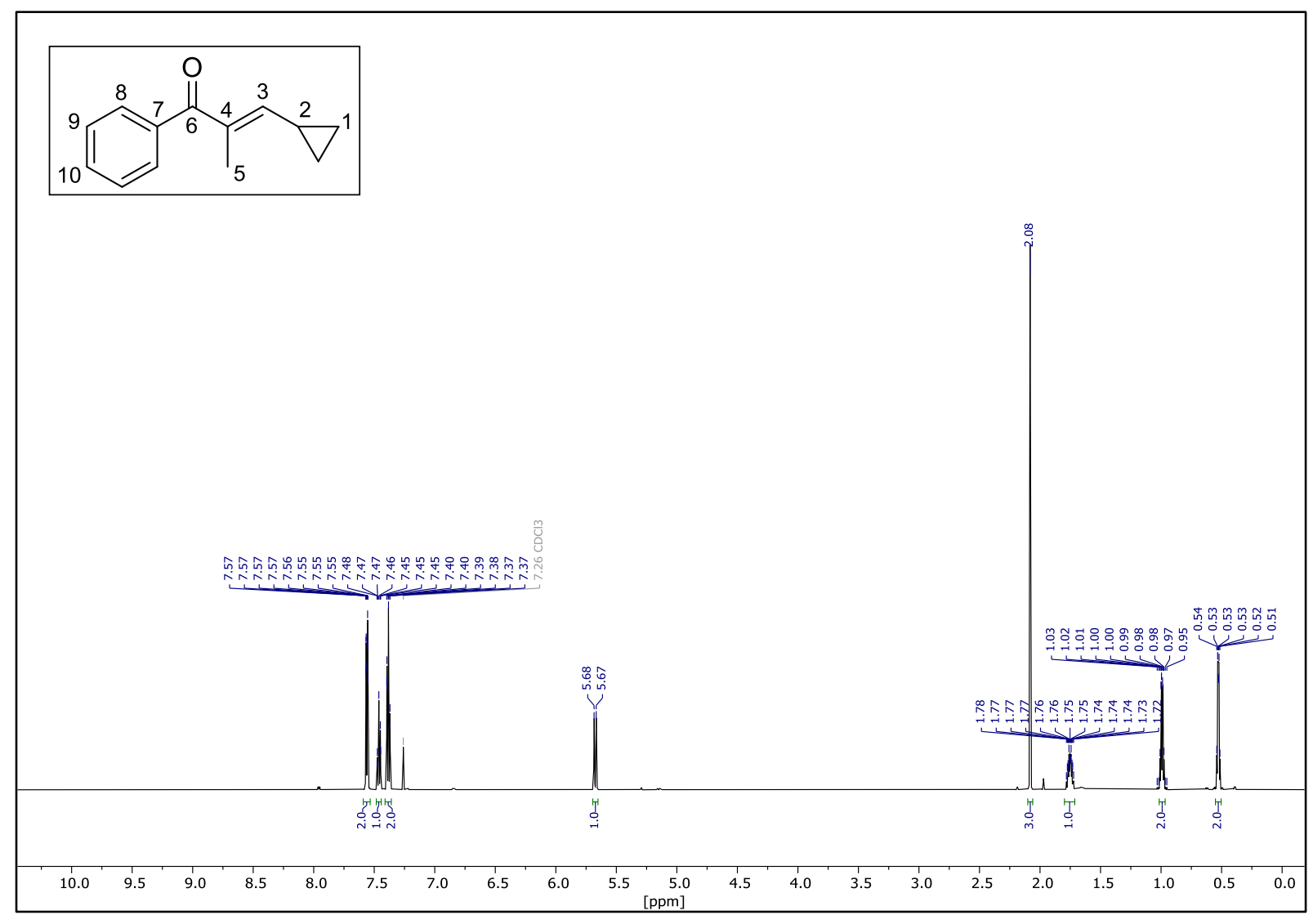

${ }^{13} \mathrm{C}$ NMR (151 MHz, $\left.\mathrm{CDCl}_{3}\right): 30$
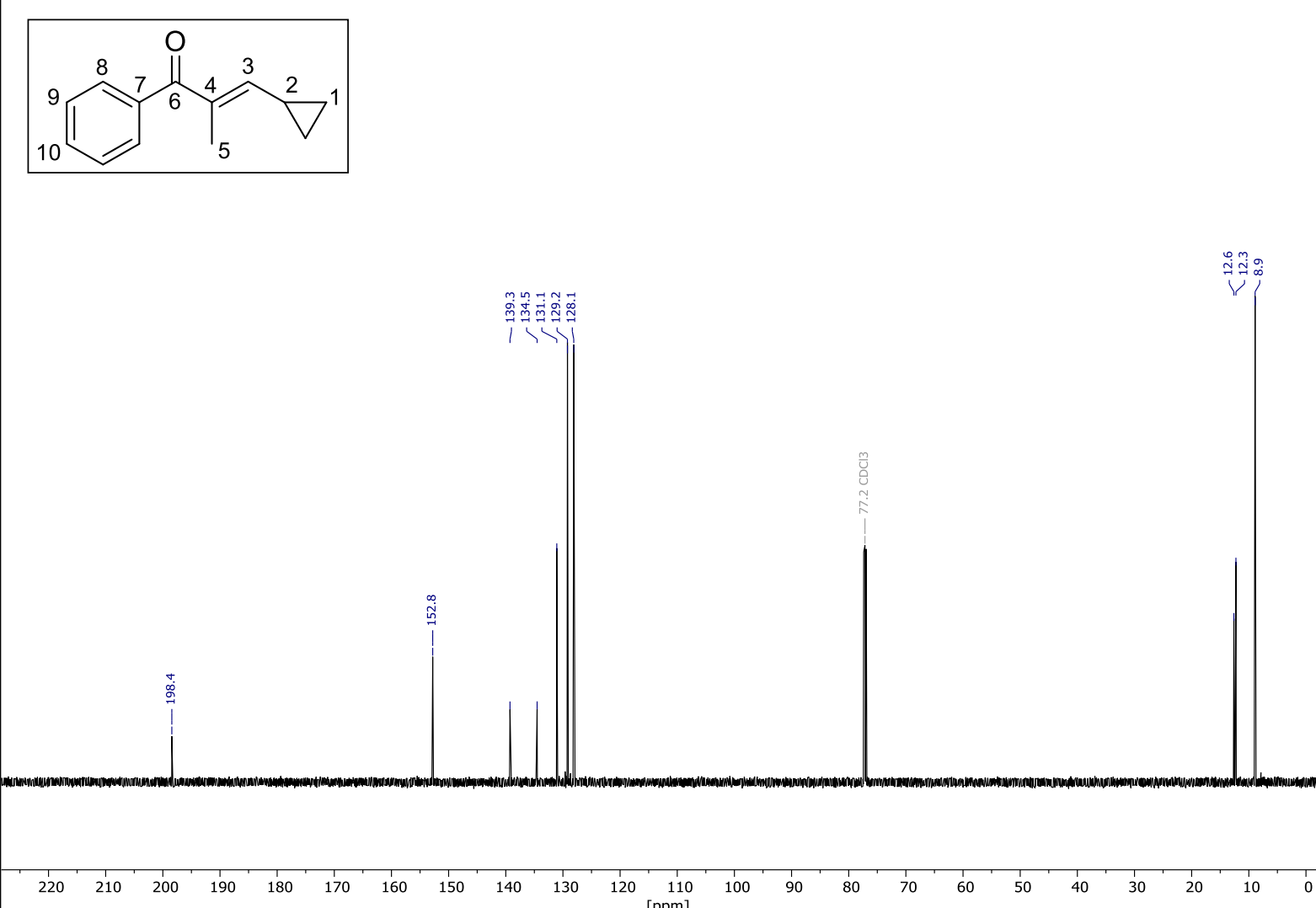
${ }^{1} \mathrm{H}$ NMR (500 MHz, $\left.\mathrm{CDCl}_{3}\right): 31$

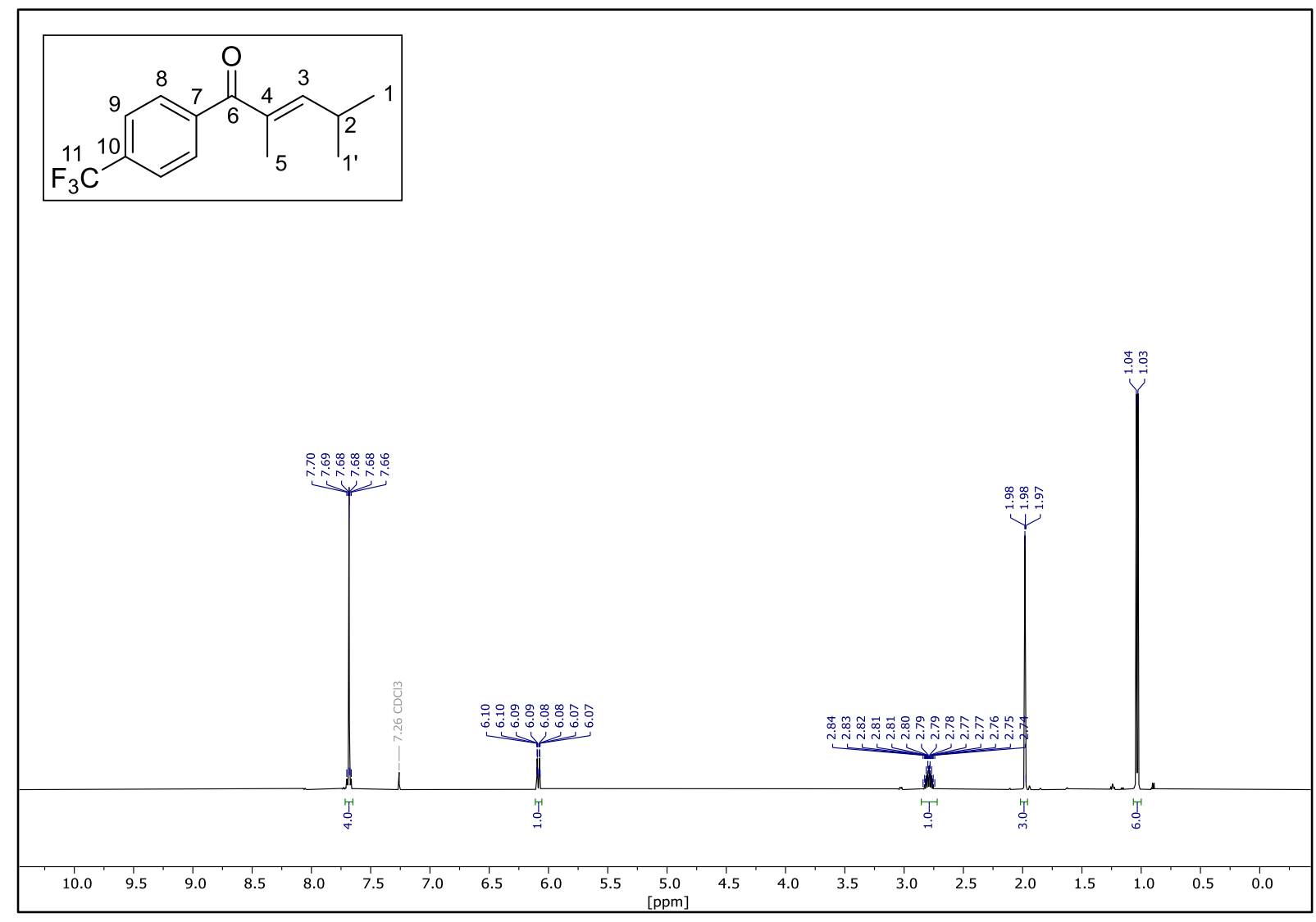

${ }^{13} \mathrm{C}$ NMR (126 MHz, $\left.\mathrm{CDCl}_{3}\right): 31$

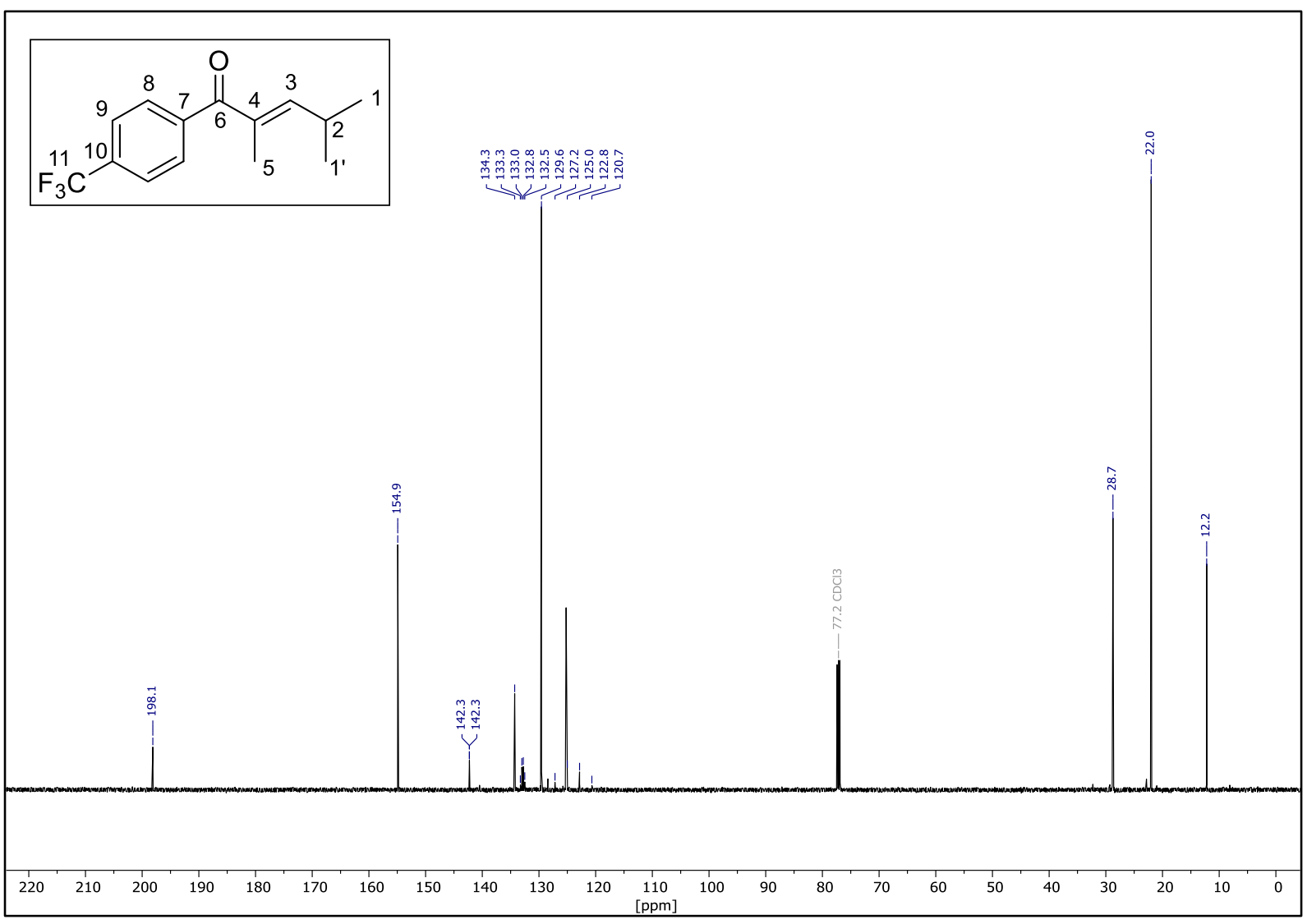


${ }^{19} \mathrm{~F} \mathrm{NMR}\left(470 \mathrm{MHz}, \mathrm{CDCl}_{3}\right): 31$

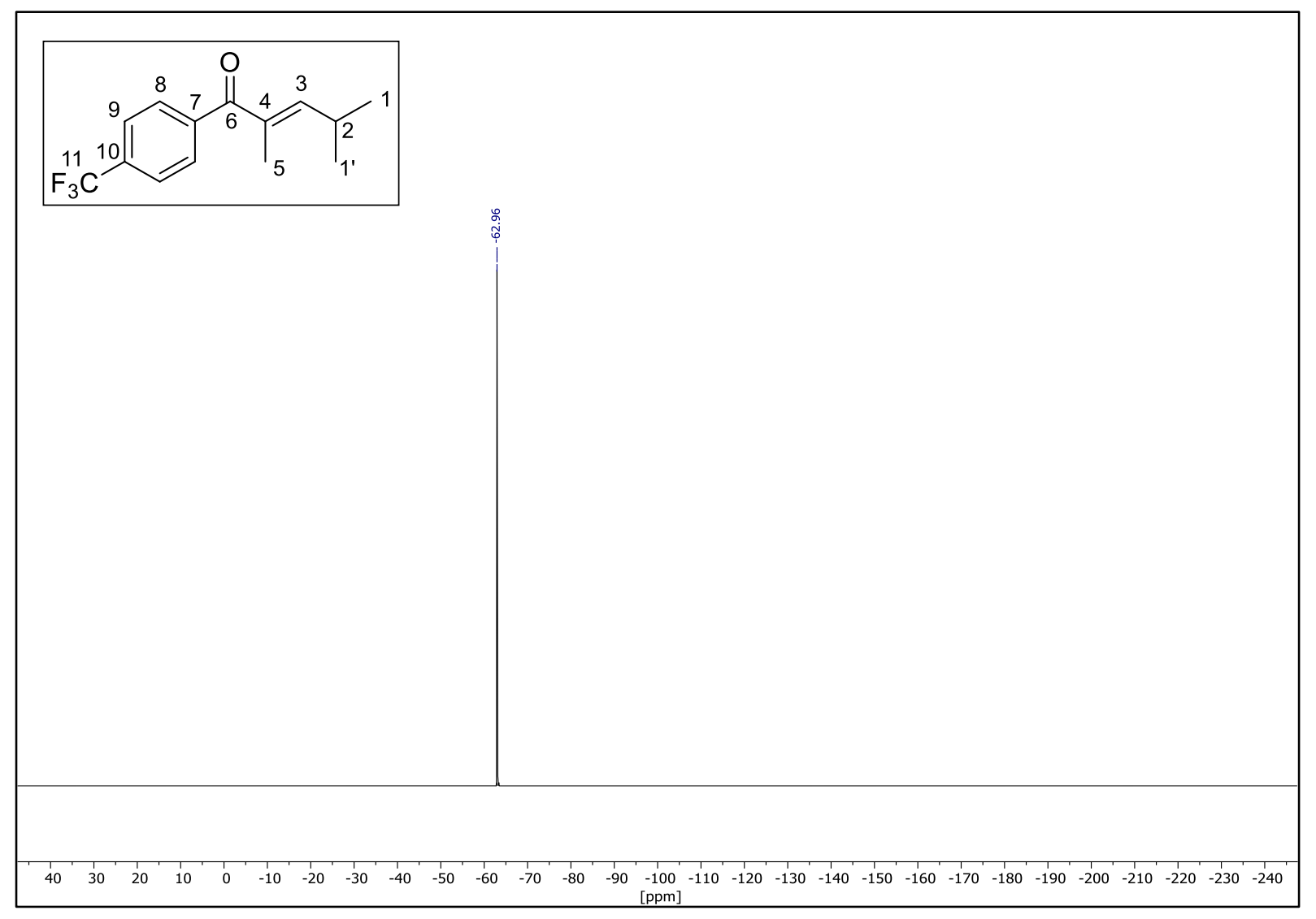

${ }^{1} \mathrm{H}$ NMR (500 MHz, $\left.\mathrm{CDCl}_{3}\right): 32$

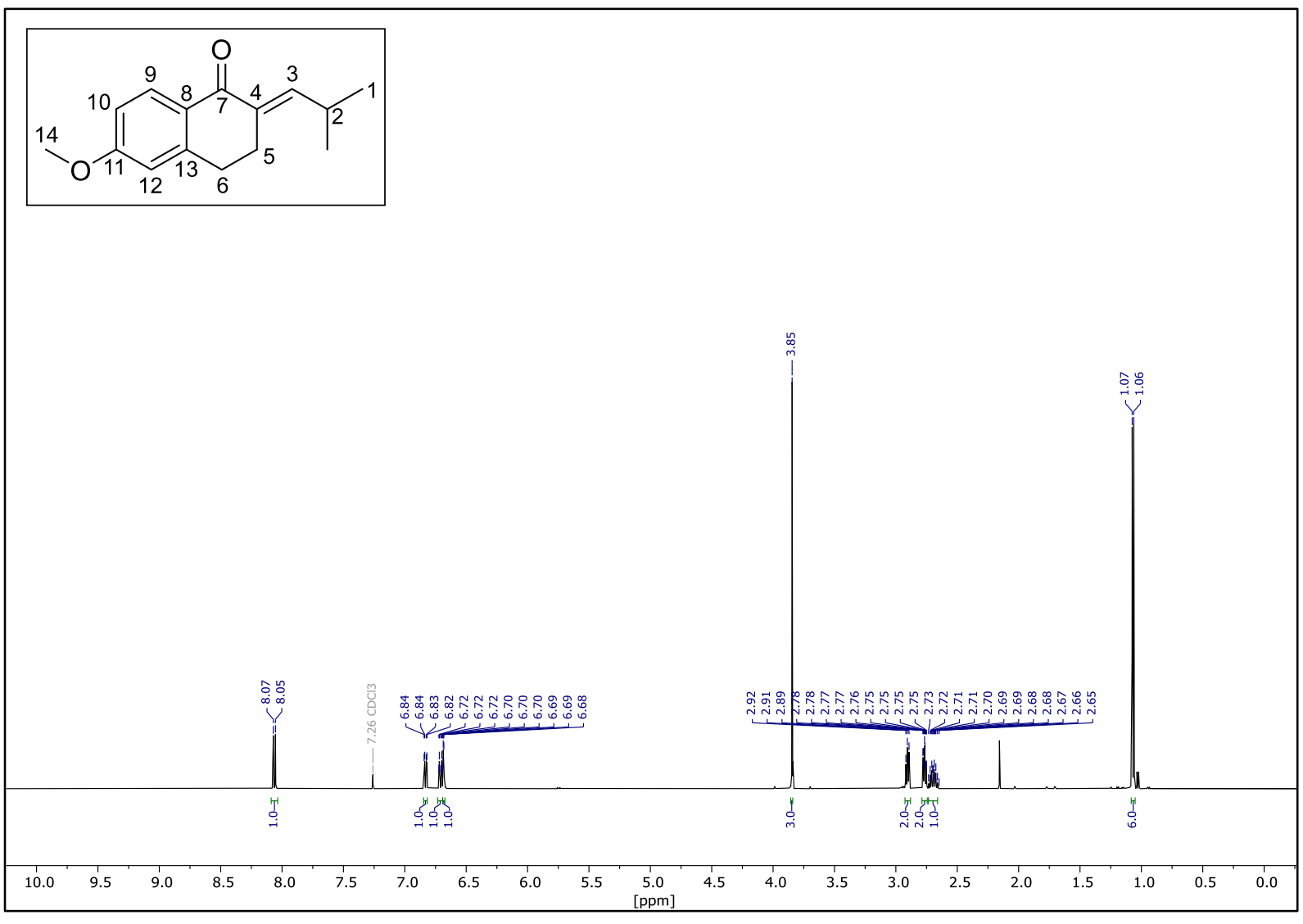


${ }^{13} \mathrm{C}$ NMR (126 MHz, $\left.\mathrm{CDCl}_{3}\right): 32$

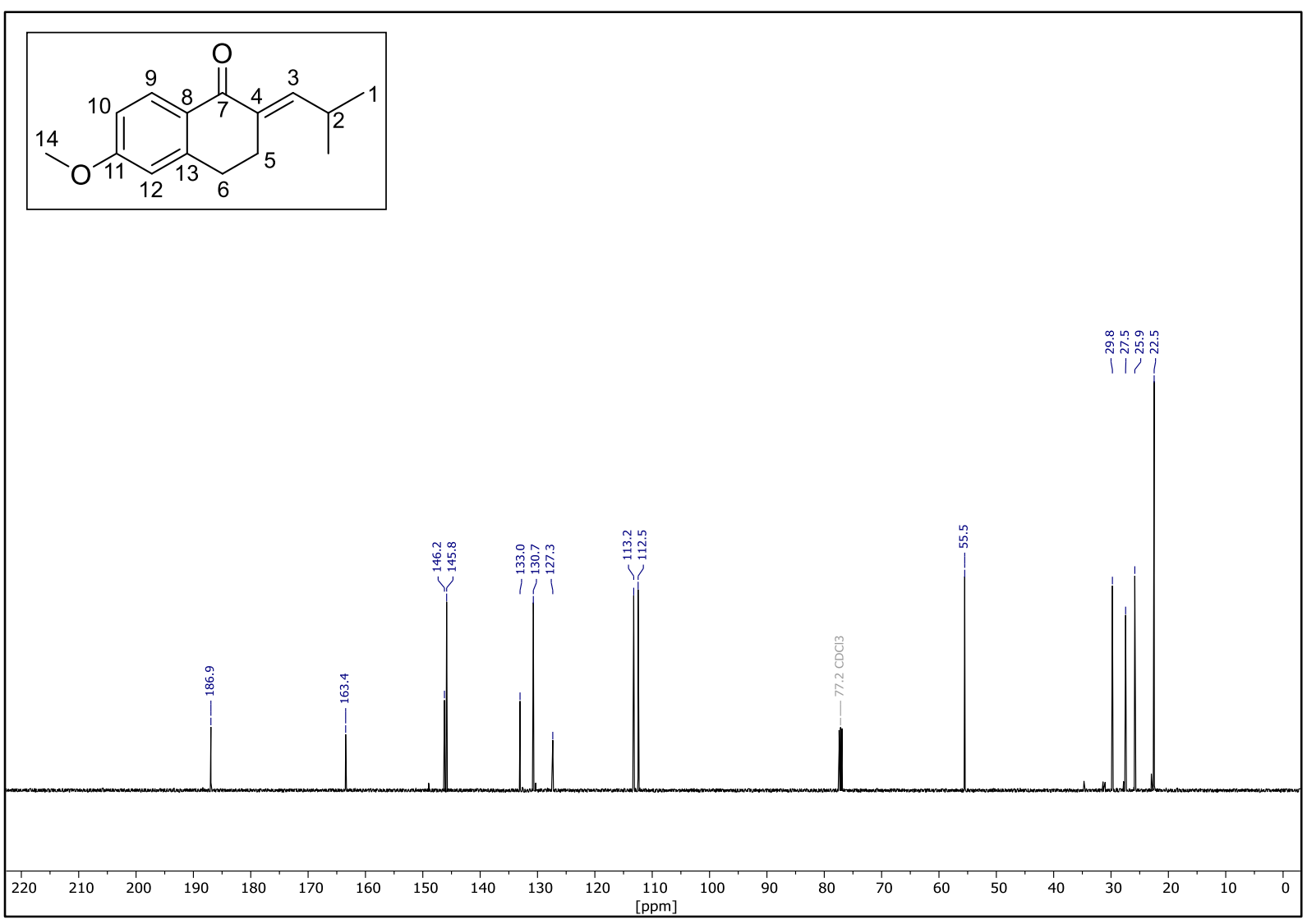

${ }^{1} \mathrm{H}$ NMR (400 MHz, $\left.\mathrm{CDCl}_{3}\right): 33$
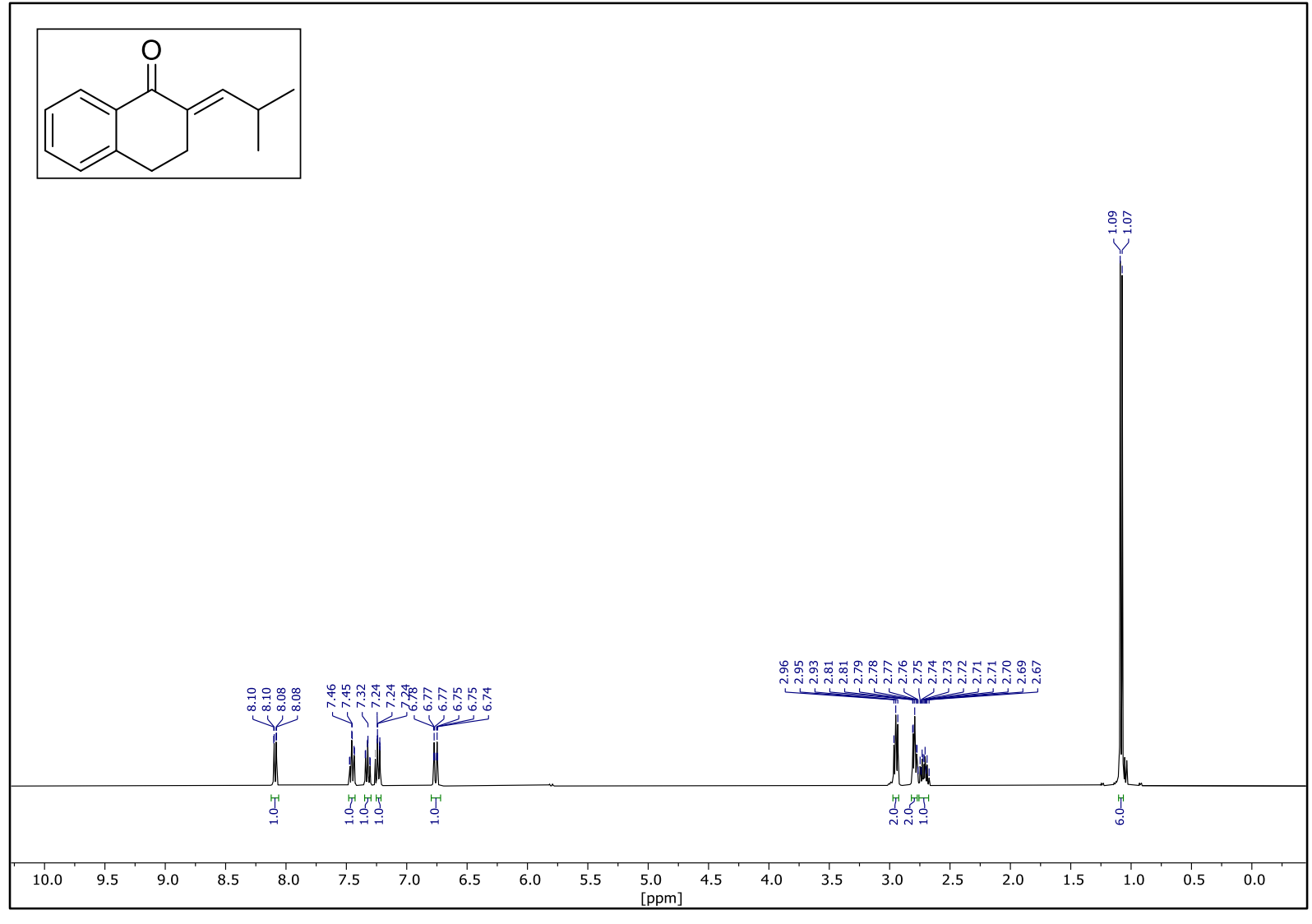
${ }^{1} \mathrm{H}$ NMR (500 MHz, $\left.\mathrm{CDCl}_{3}\right): 34$

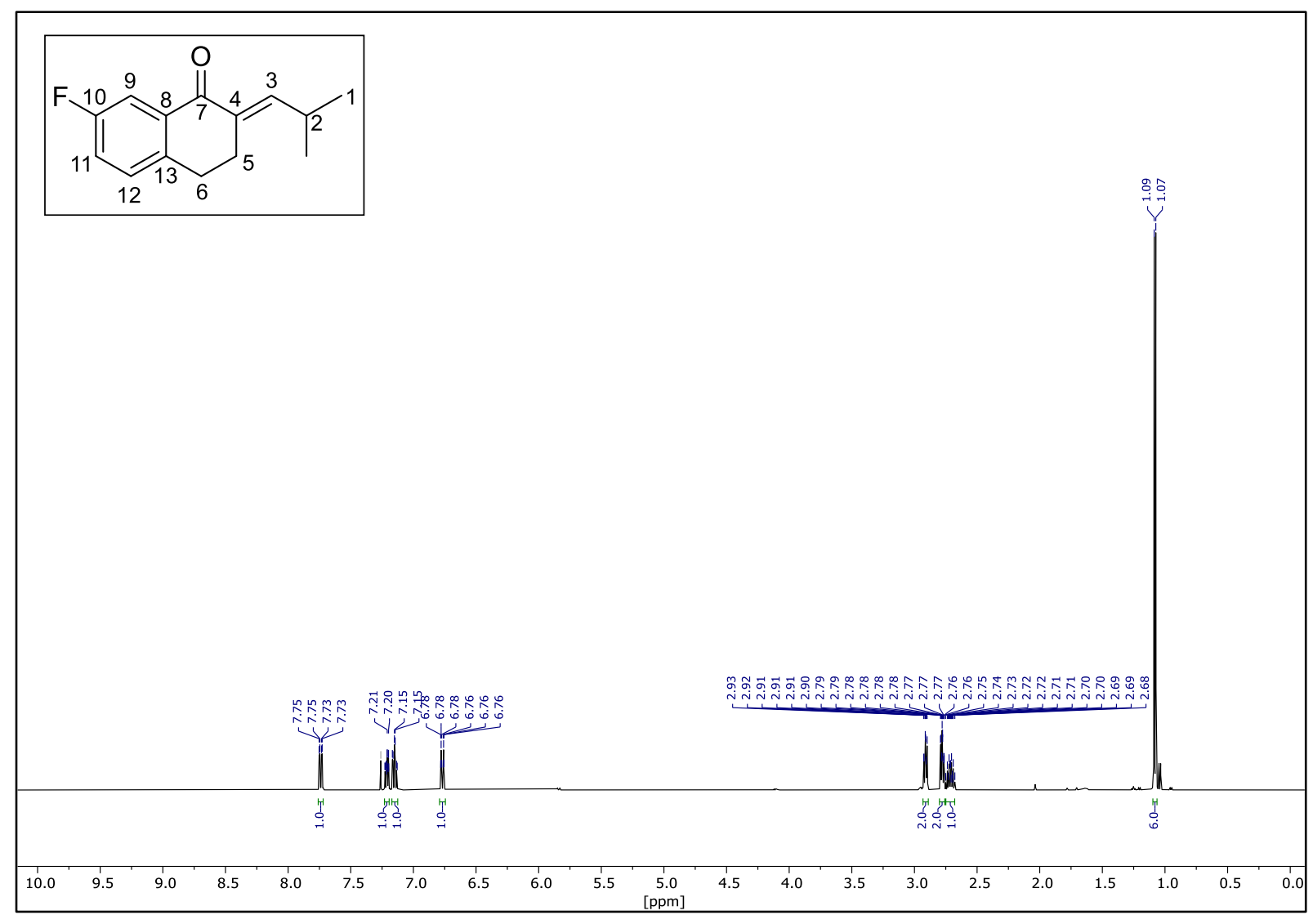

${ }^{13} \mathrm{C}$ NMR (126 MHz, $\left.\mathrm{CDCl}_{3}\right): 34$

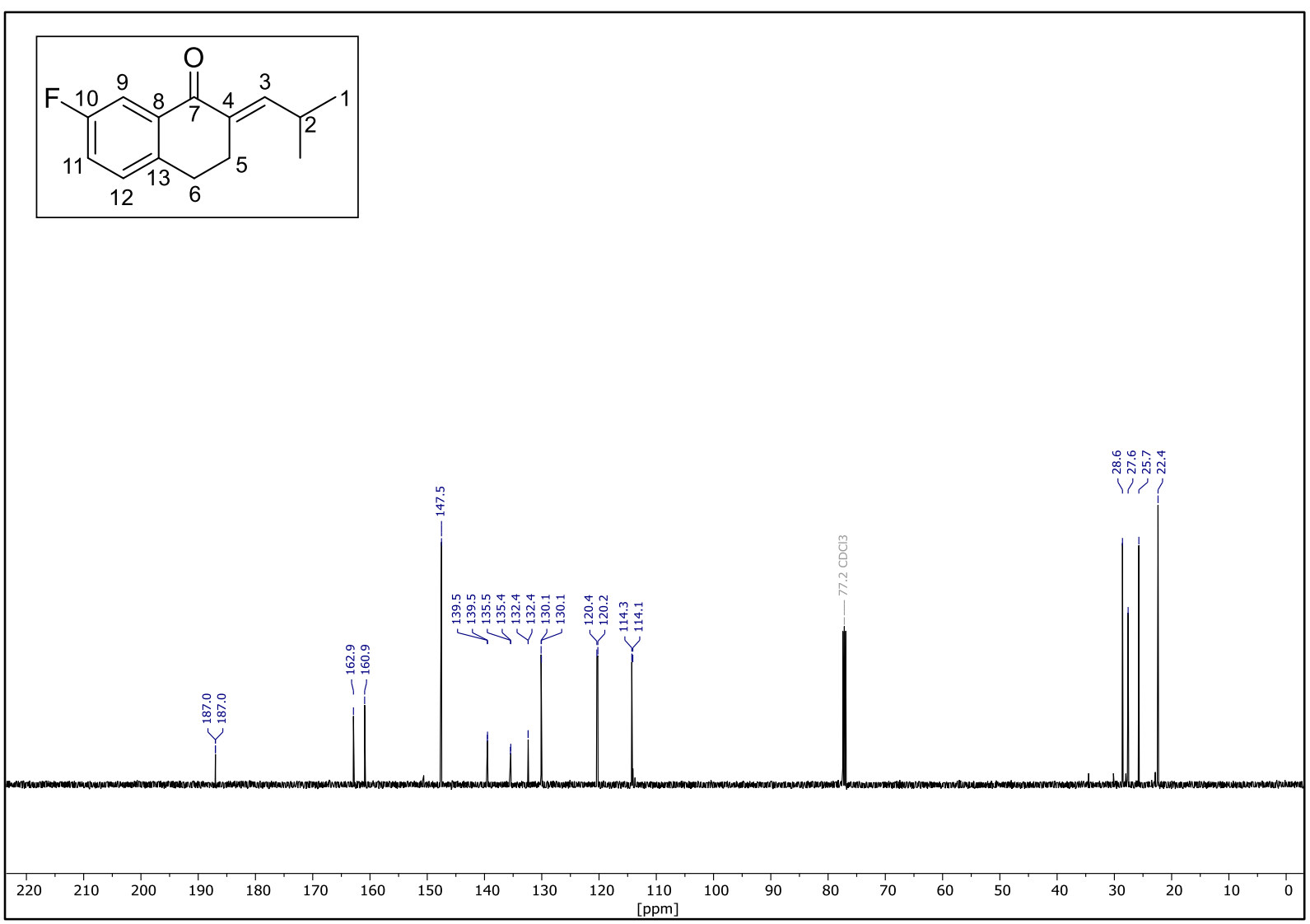


${ }^{19}$ F NMR (470 MHz, $\left.\mathrm{CDCl}_{3}\right): 34$

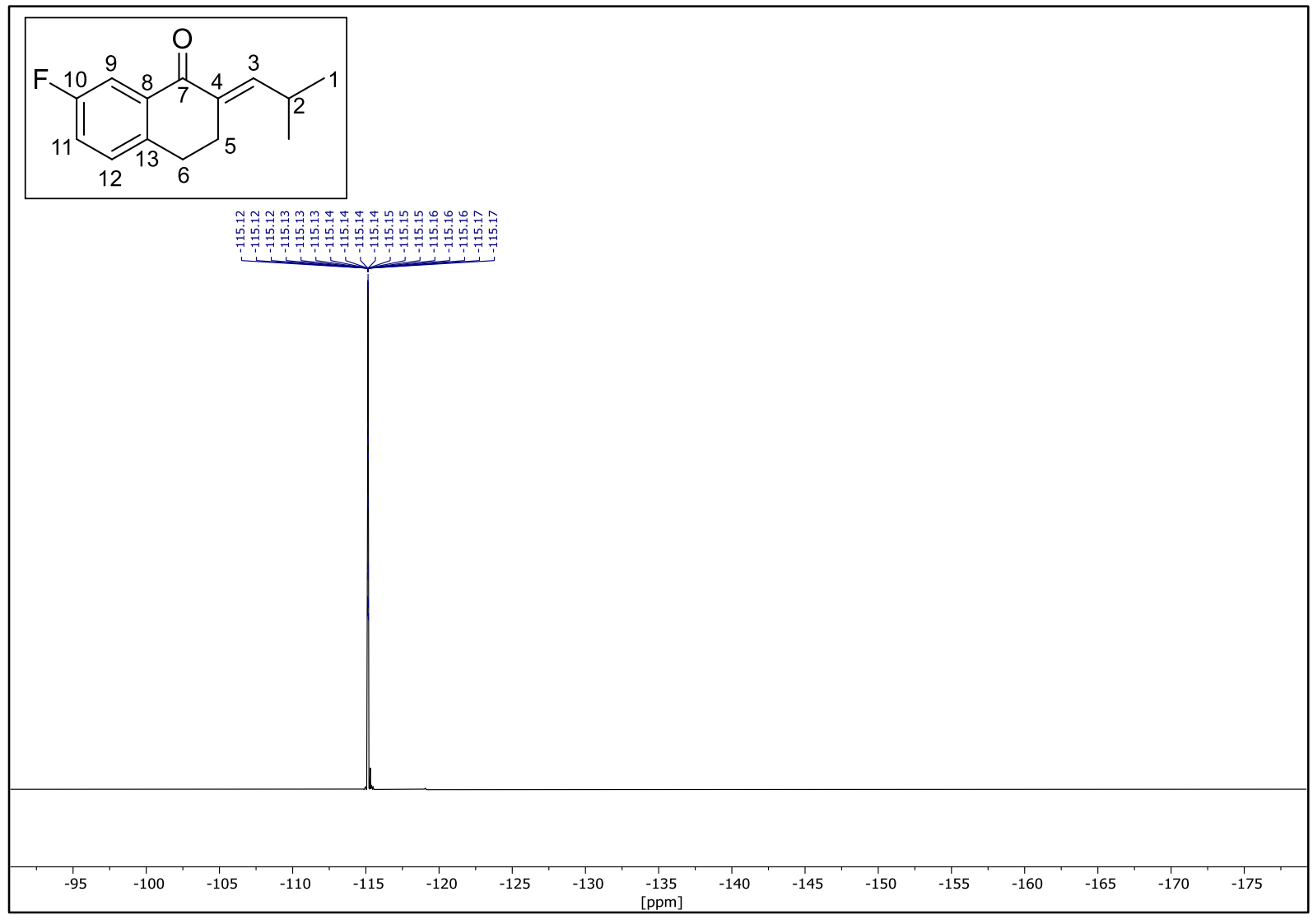

${ }^{1} \mathrm{H}$ NMR (600 MHz, $\left.\mathrm{CDCl}_{3}\right): 35$

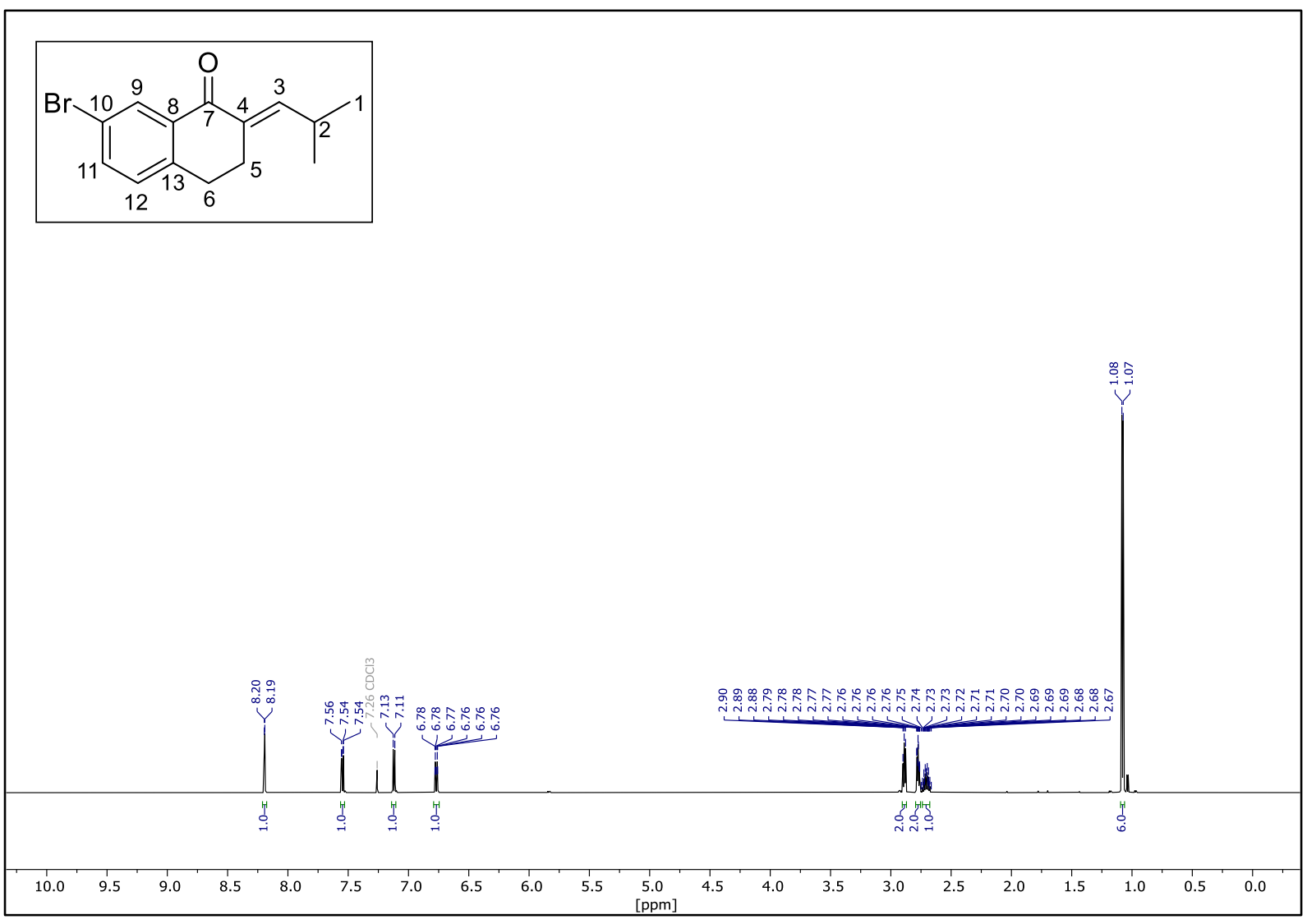


${ }^{13} \mathrm{C}$ NMR (151 MHz, $\left.\mathrm{CDCl}_{3}\right): 35$

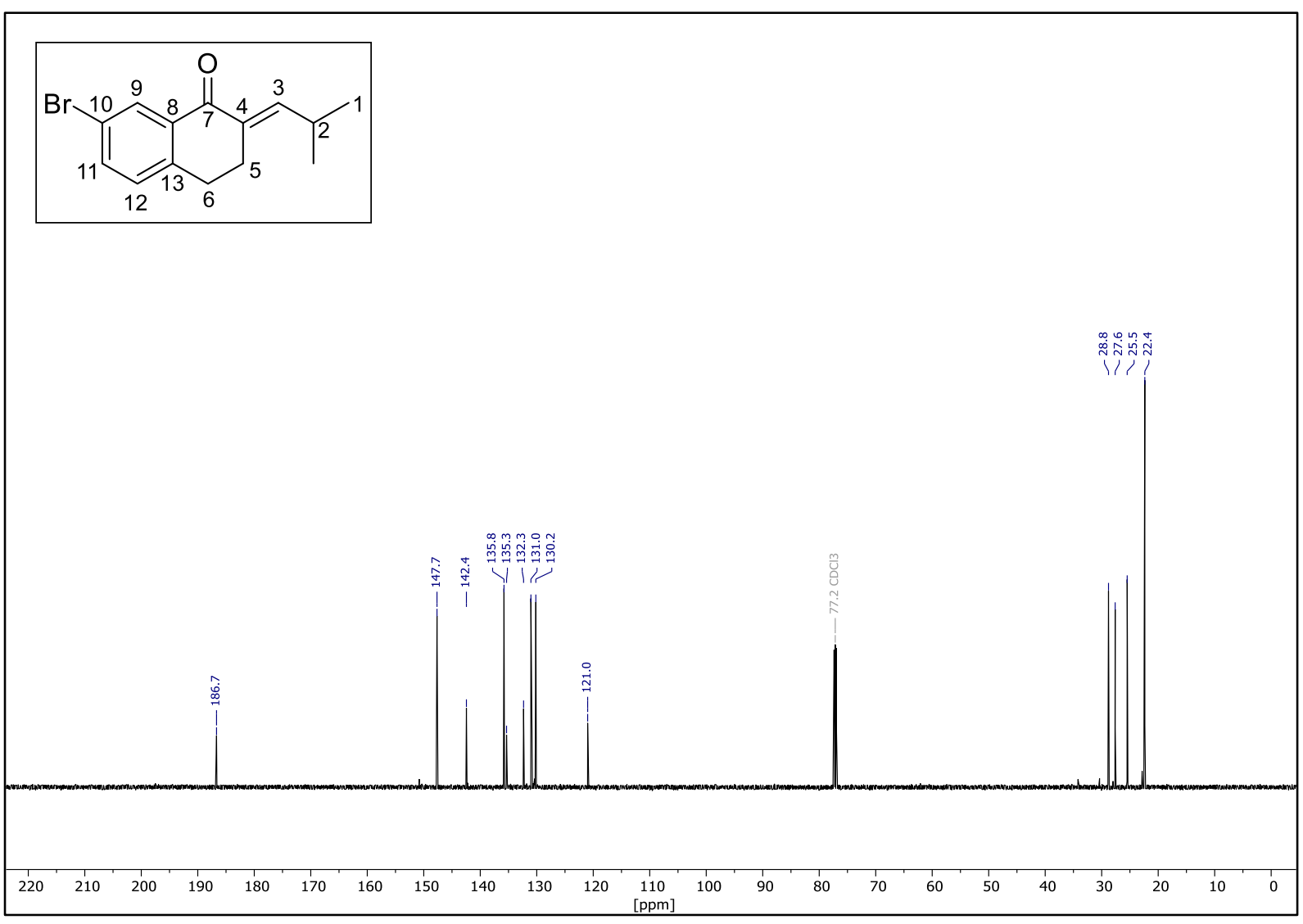

${ }^{1} \mathrm{H}$ NMR (600 MHz, $\left.\mathrm{CDCl}_{3}\right): 36$

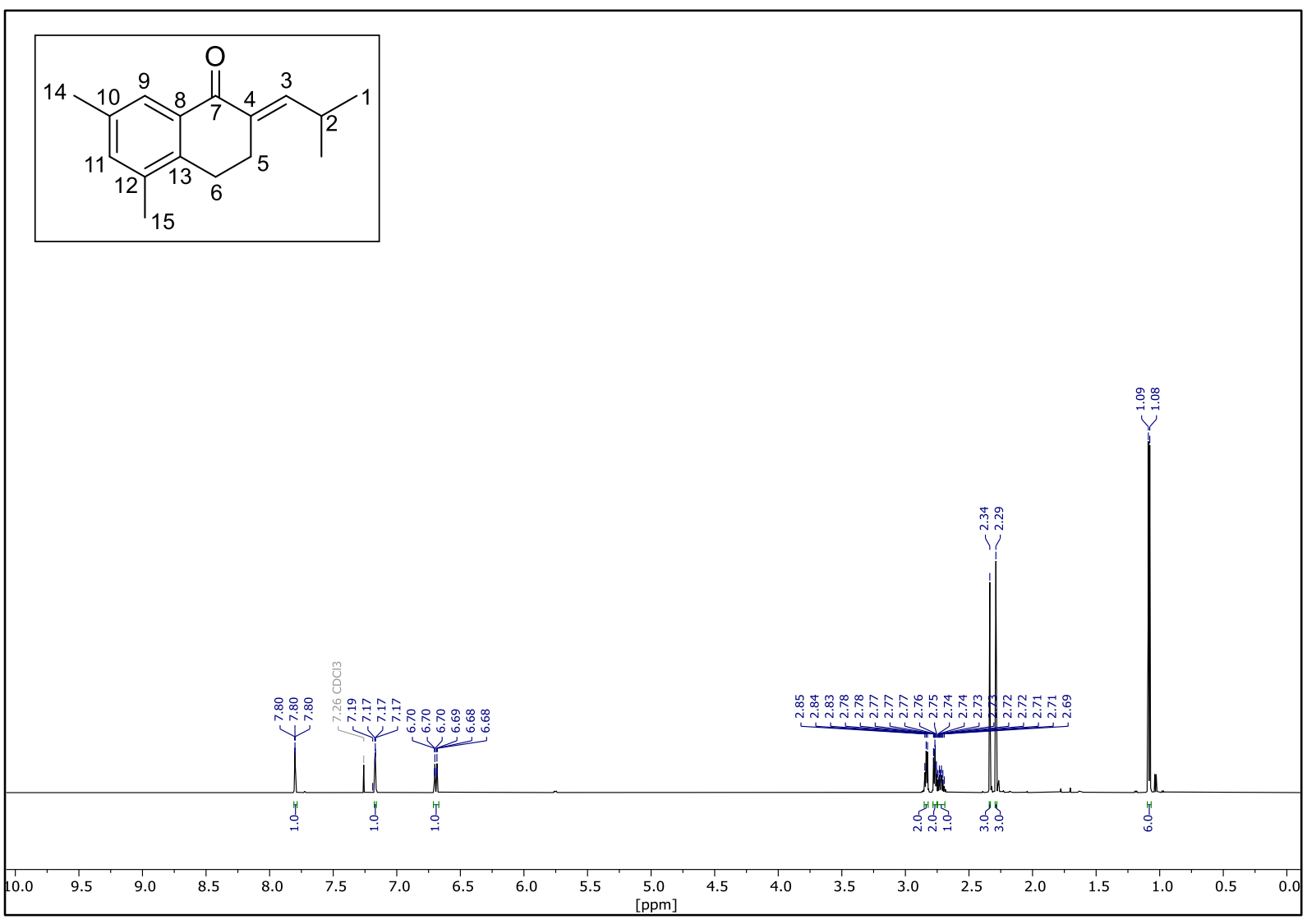


${ }^{13} \mathrm{C}$ NMR (151 MHz, $\left.\mathrm{CDCl}_{3}\right): 36$

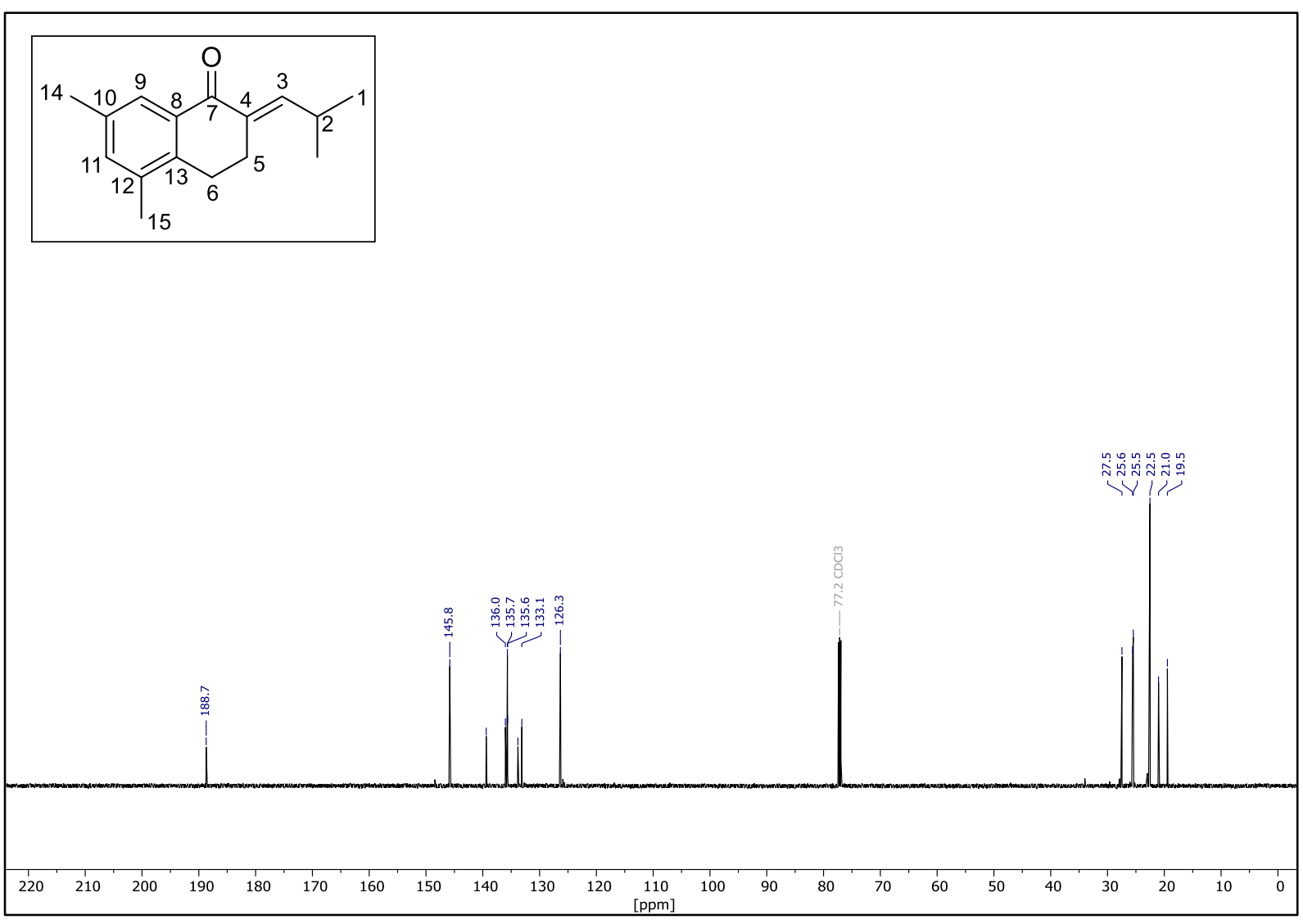

${ }^{1} \mathrm{H}$ NMR (500 MHz, $\mathrm{CDCl}_{3}$ ): 37

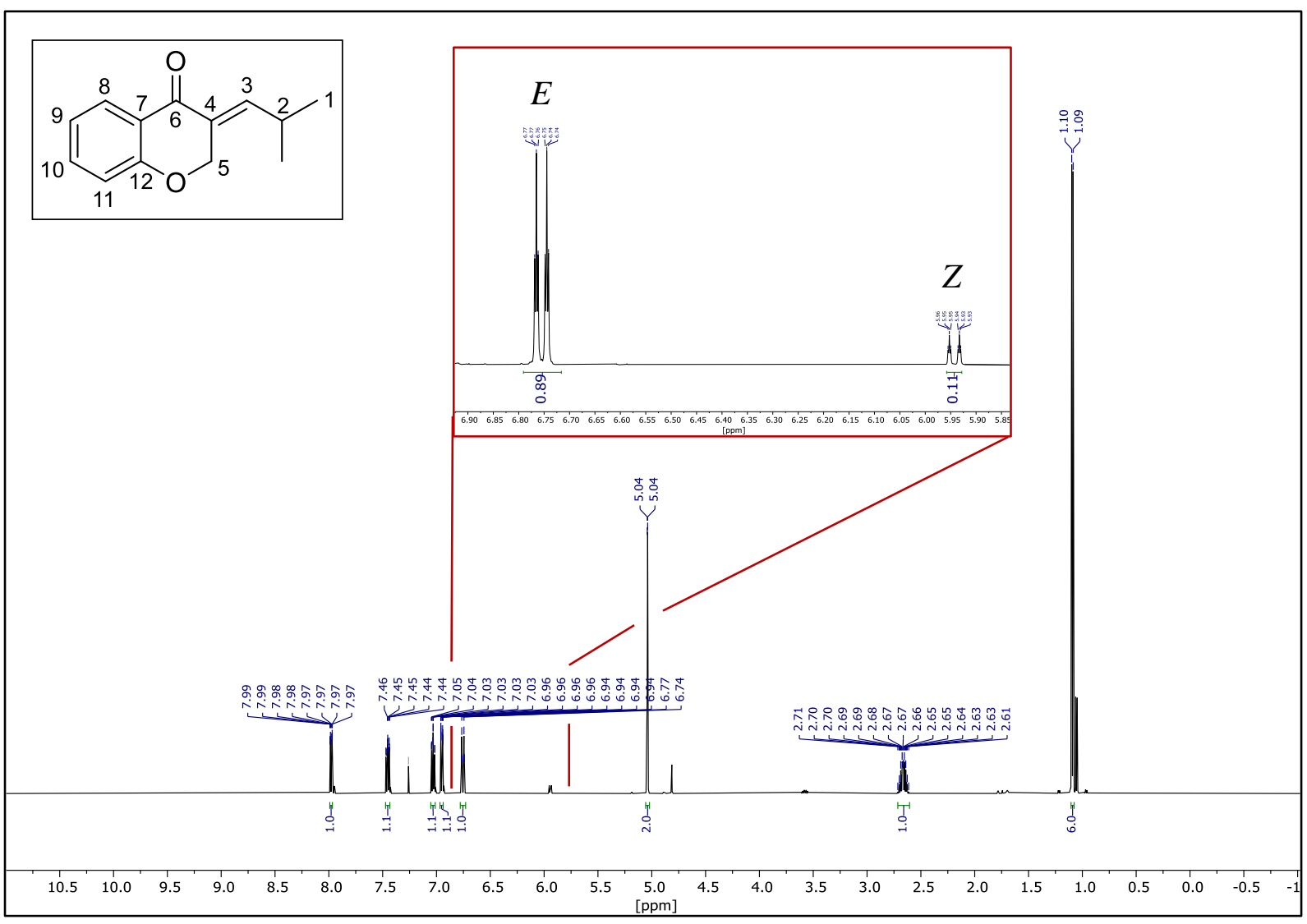


${ }^{13} \mathrm{C}$ NMR (126 MHz, $\left.\mathrm{CDCl}_{3}\right): 37$

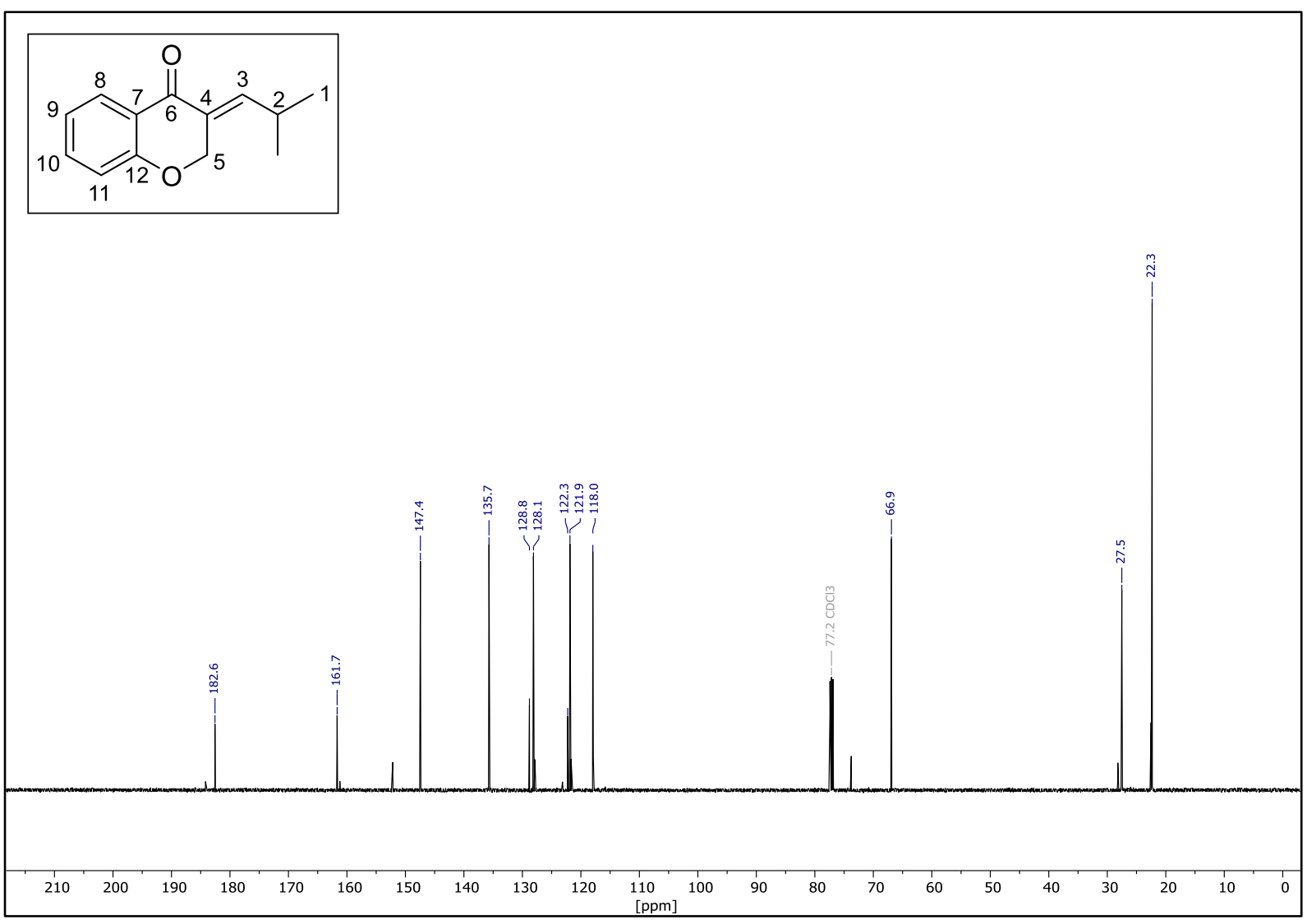

${ }^{1} \mathrm{H},{ }^{1} \mathrm{H} \operatorname{COSY}\left(500 \mathrm{MHz}, \mathrm{CDCl}_{3}\right): 37$

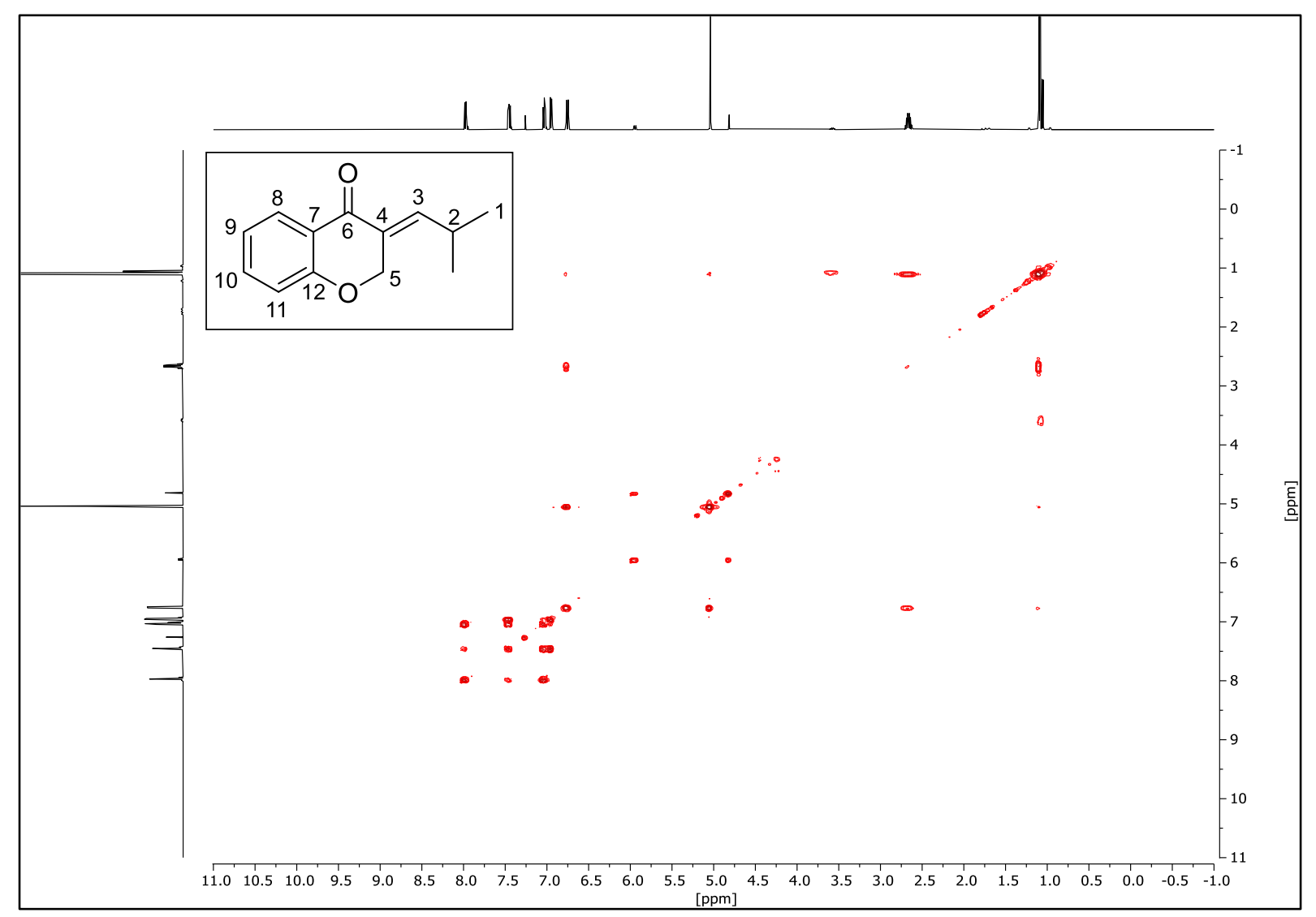


${ }^{1} \mathrm{H},{ }^{13} \mathrm{C}$ HMBC $\left(500 \mathrm{MHz}, \mathrm{CDCl}_{3}\right): 37$

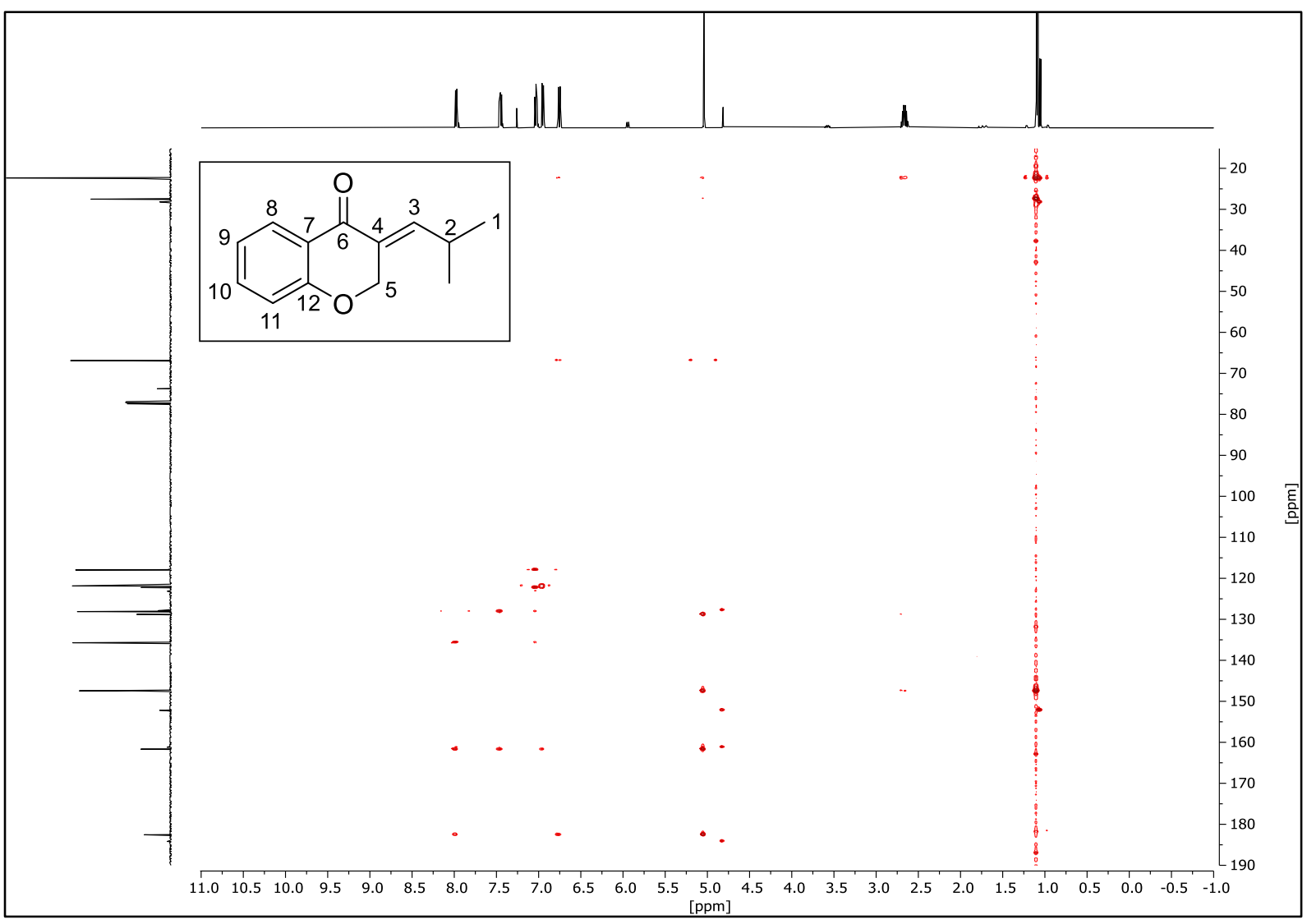

${ }^{1} \mathrm{H},{ }^{13} \mathrm{C}$ HSQC (500 MHz, $\left.\mathrm{CDCl}_{3}\right): 37$

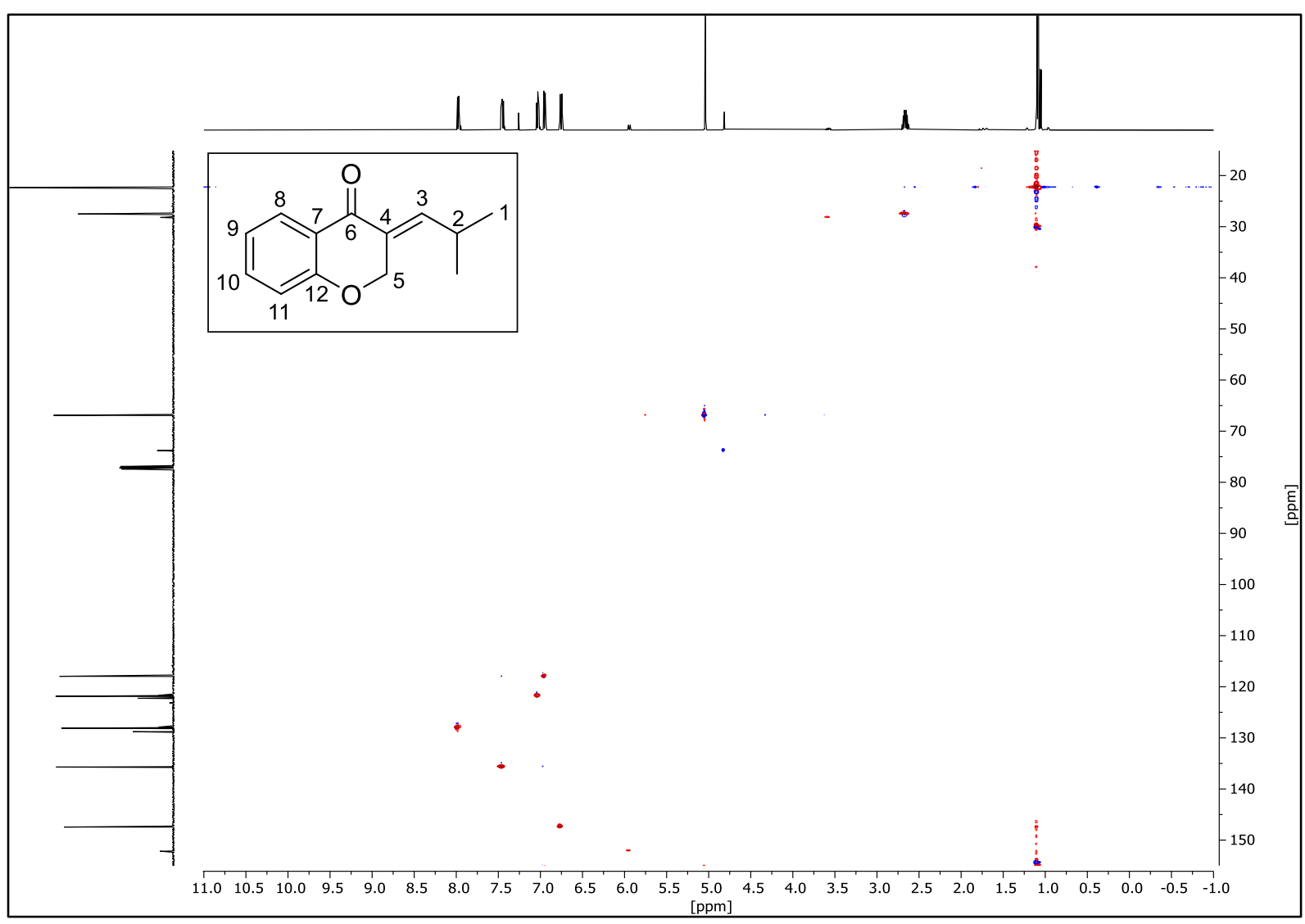


1D NOESY (500 MHz, $\left.\mathrm{CDCl}_{3}\right): 37$

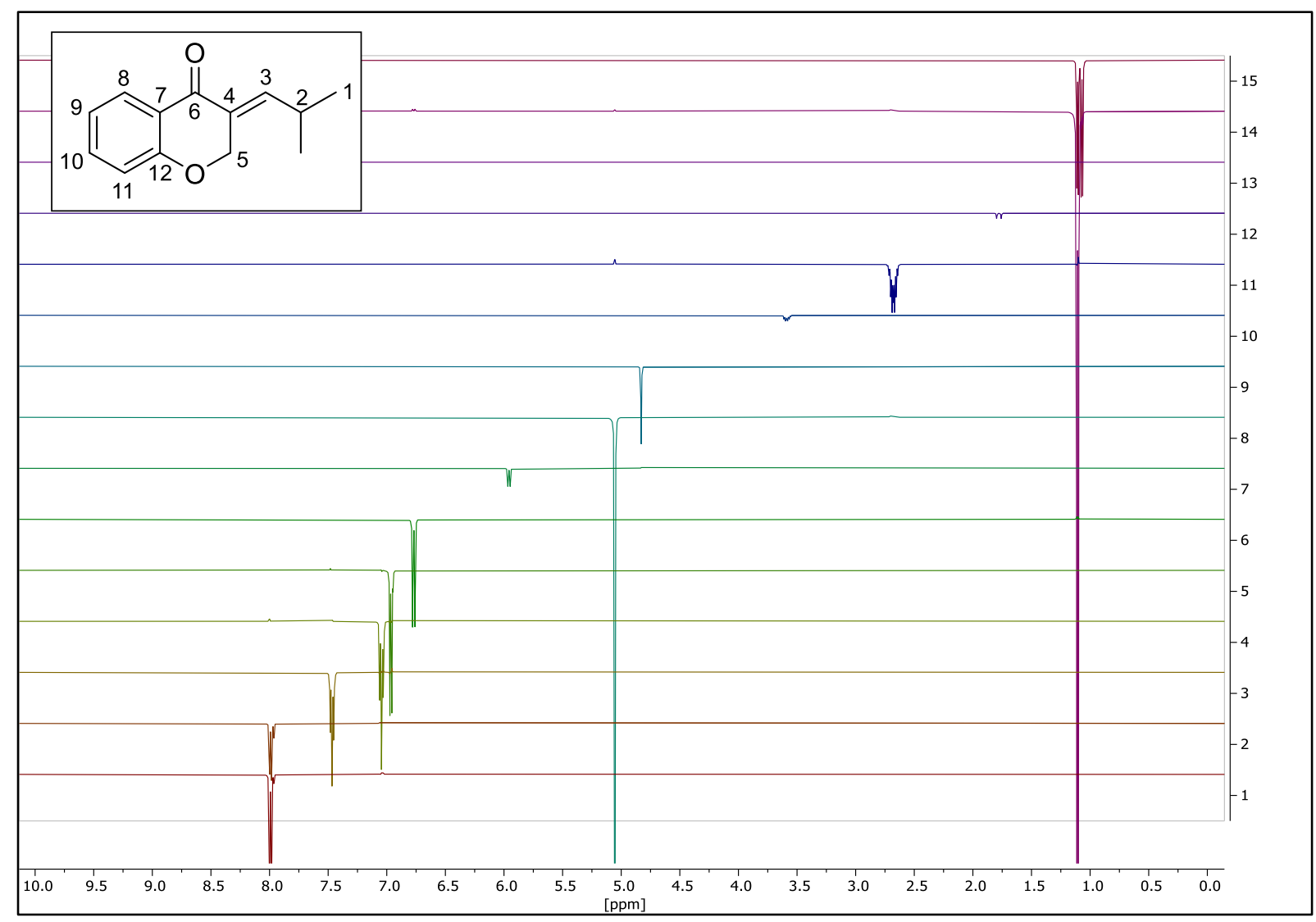

${ }^{1} \mathrm{H}$ NMR (400 MHz, $\left.\mathrm{CDCl}_{3}\right): 38$

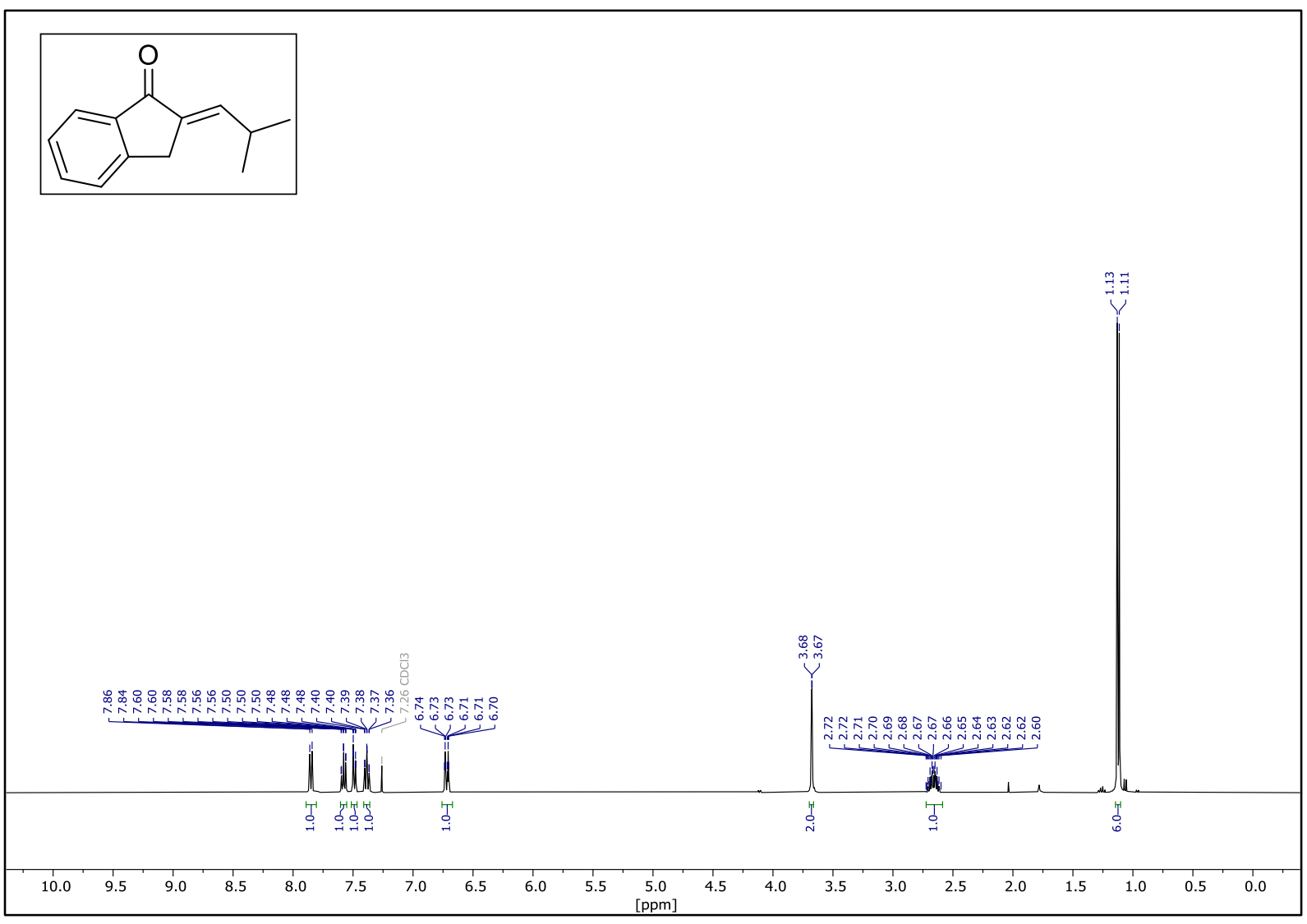


${ }^{1} \mathrm{H}$ NMR (500 MHz, $\left.\mathrm{CDCl}_{3}\right): 39$

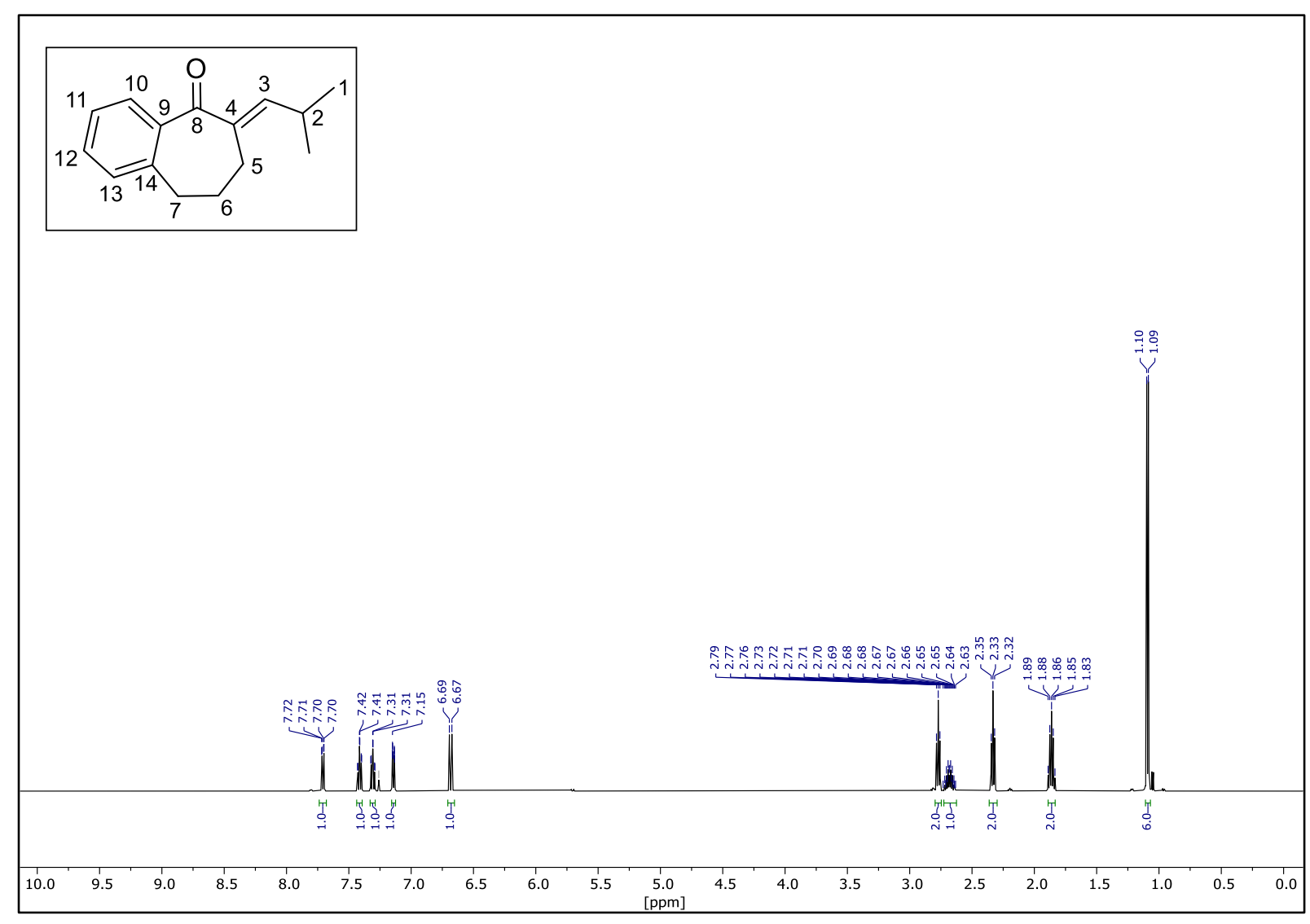

${ }^{13} \mathrm{C}$ NMR (126 MHz, $\left.\mathrm{CDCl}_{3}\right): 39$

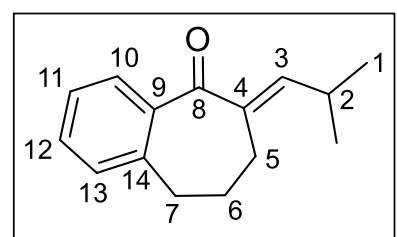

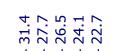


${ }^{1} \mathrm{H}$ NMR $\left(600 \mathrm{MHz}, \mathrm{CDCl}_{3}\right): 40$

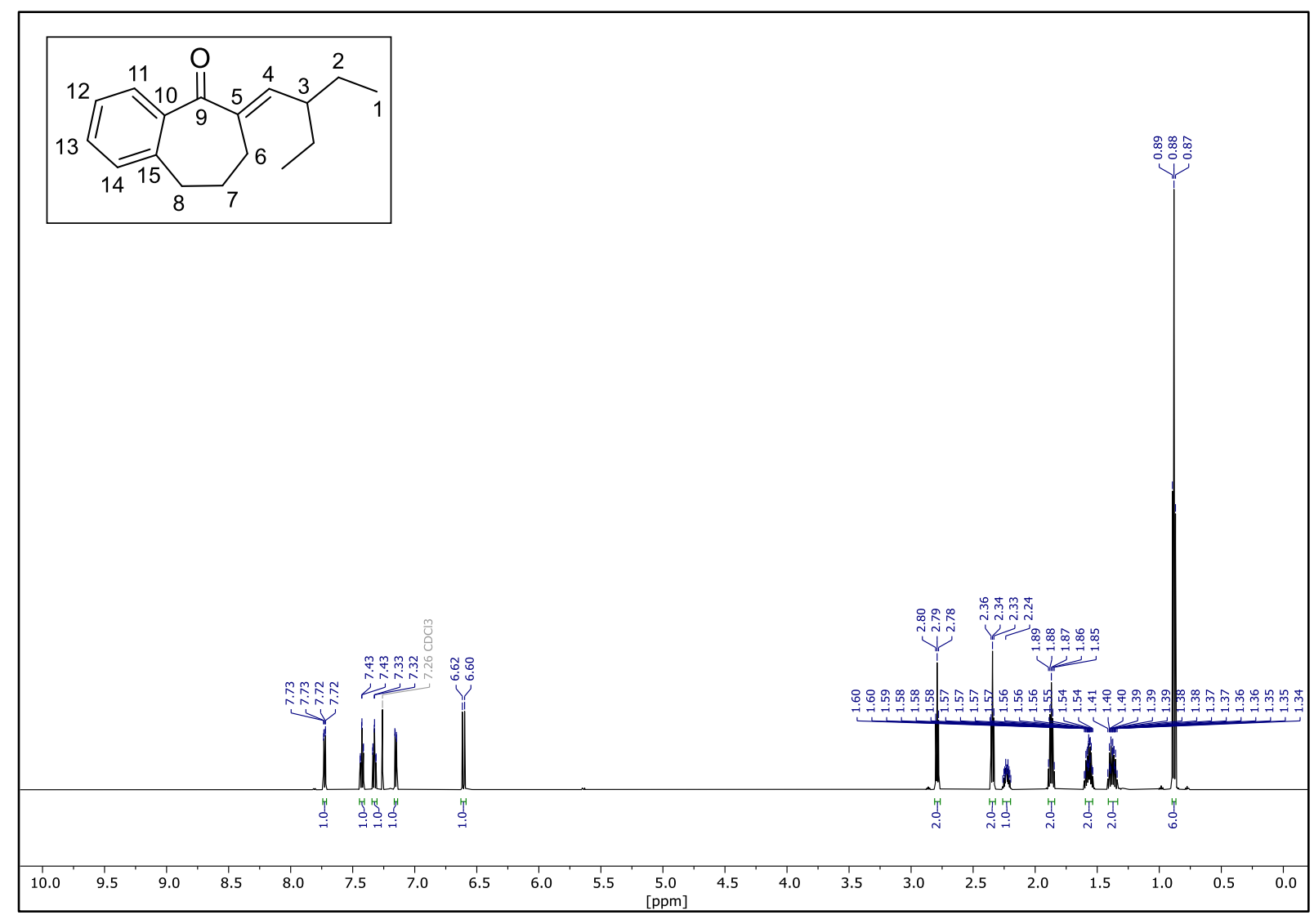

${ }^{13} \mathrm{C}$ NMR (151 MHz, $\left.\mathrm{CDCl}_{3}\right): 40$

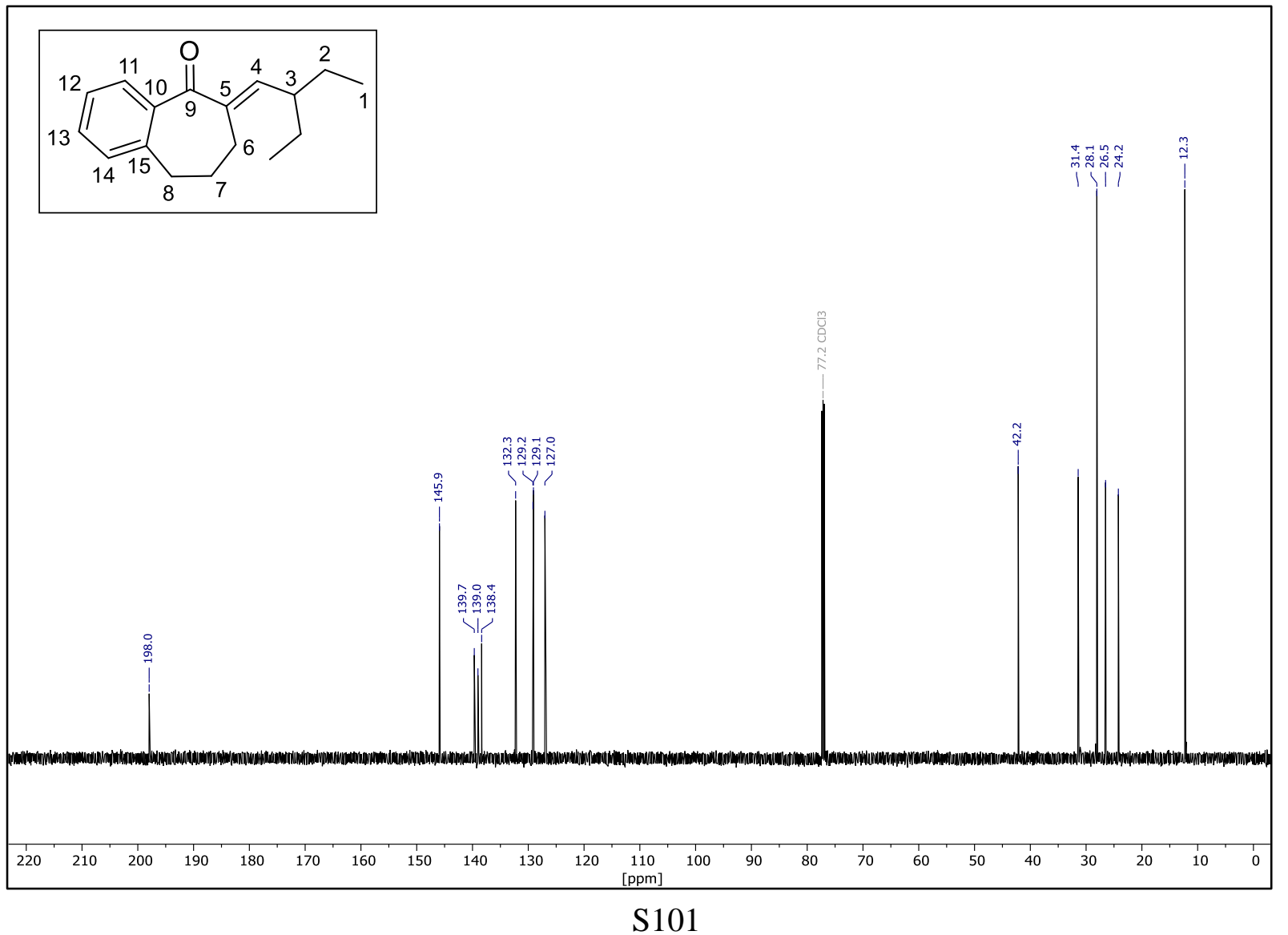


${ }^{1} \mathrm{H}$ NMR (600 MHz, $\left.\mathrm{CDCl}_{3}\right): 41$

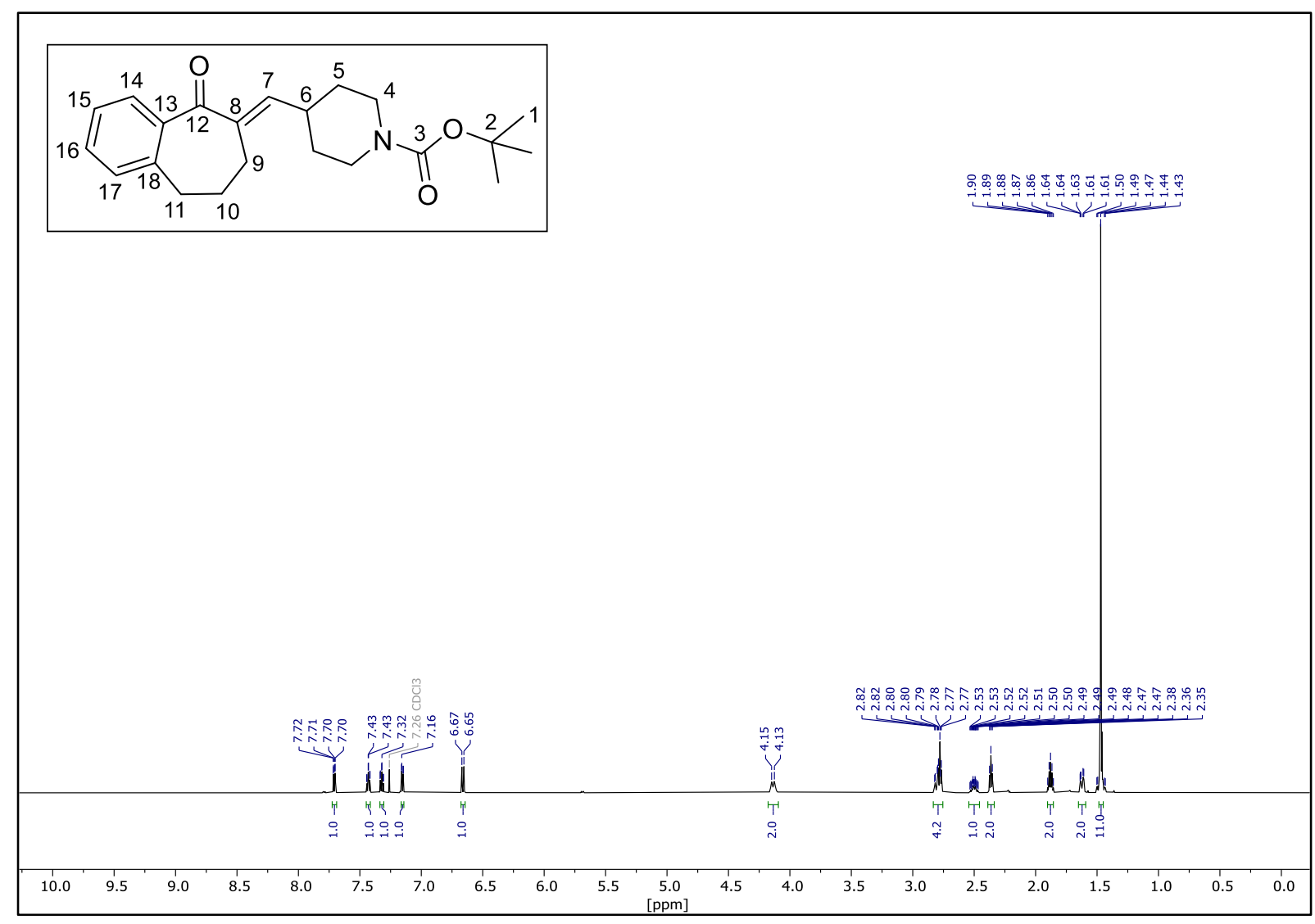

${ }^{13} \mathrm{C}$ NMR (151 MHz, $\left.\mathrm{CDCl}_{3}\right): 41$

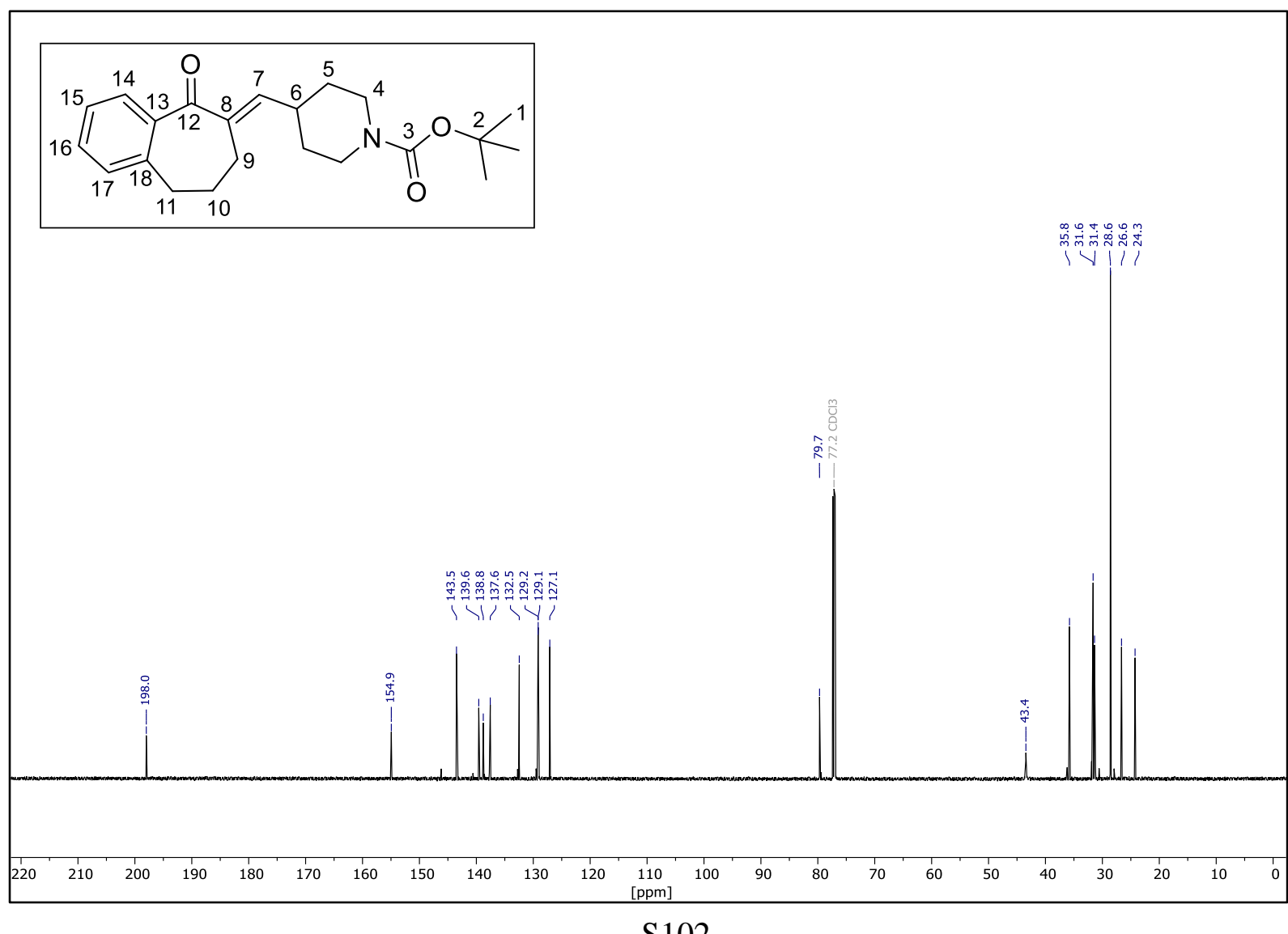


${ }^{1} \mathrm{H}$ NMR (600 MHz, $\left.\mathrm{CDCl}_{3}\right): 42$
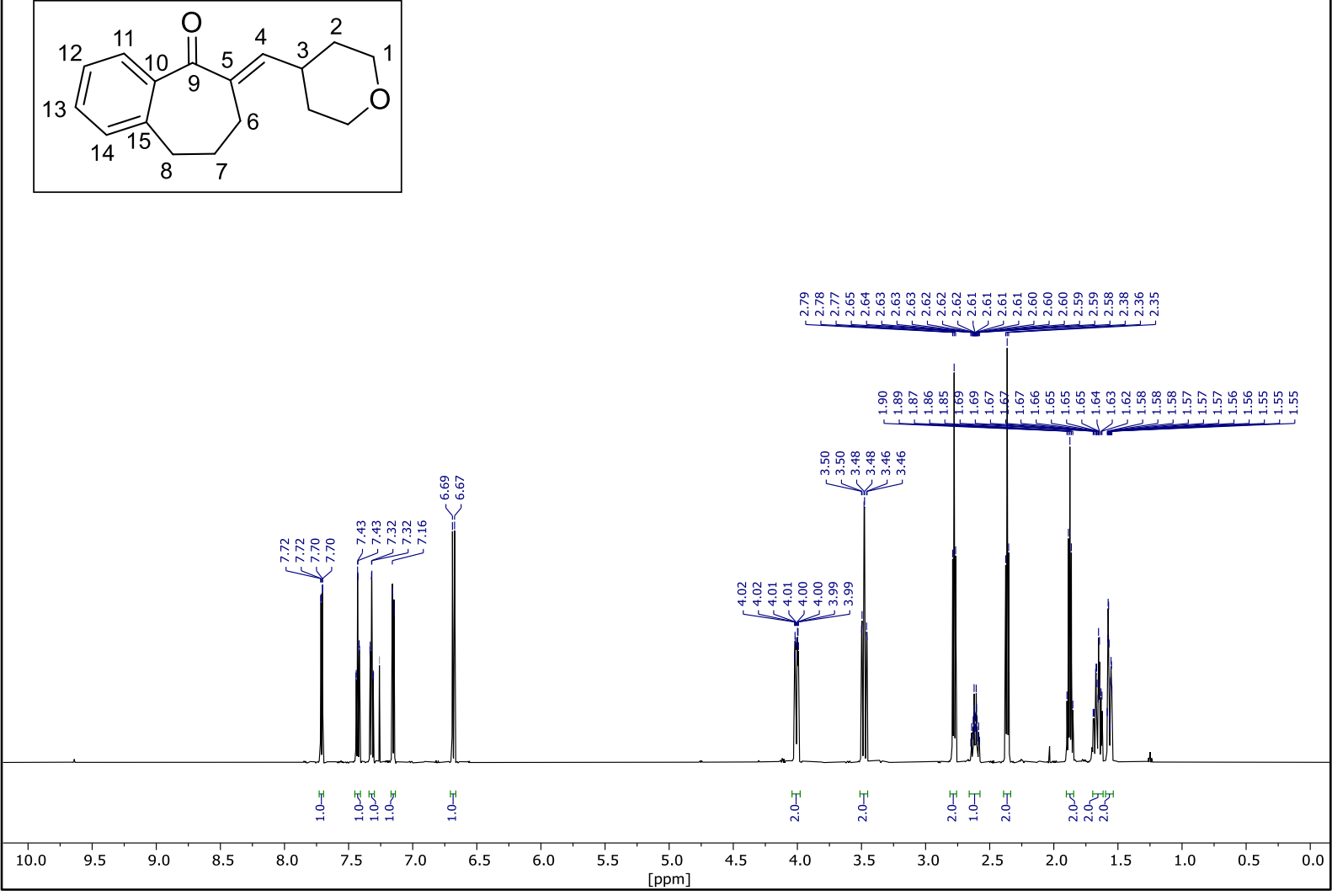

${ }^{13} \mathrm{C}$ NMR $\left(151 \mathrm{MHz}, \mathrm{CDCl}_{3}\right): 42$

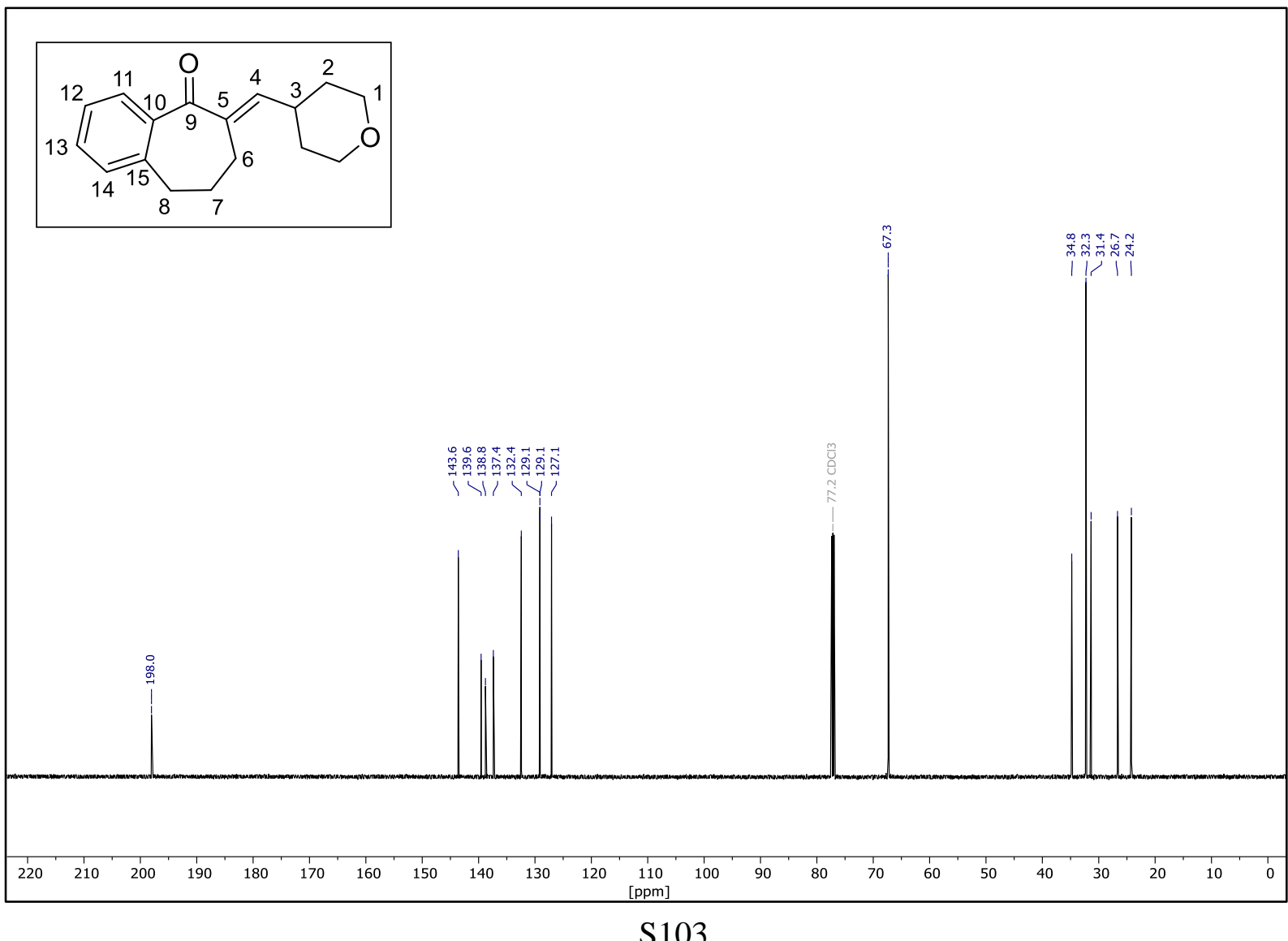


${ }^{1} \mathrm{H}$ NMR (400 MHz, $\left.\mathrm{CDCl}_{3}\right): 43$

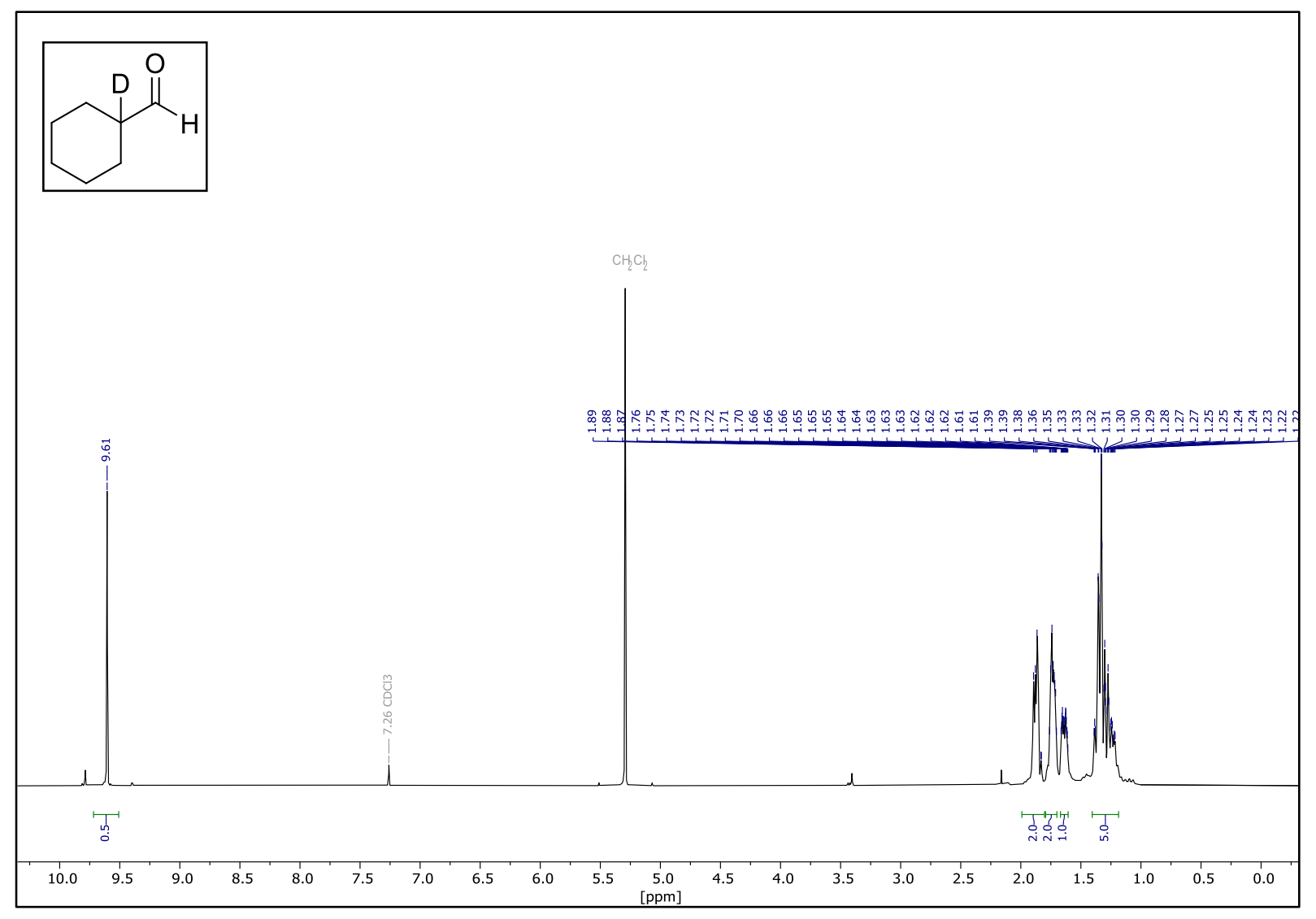

${ }^{1} \mathrm{H}$ NMR (500 MHz, $\left.\mathrm{CDCl}_{3}\right): d-22$

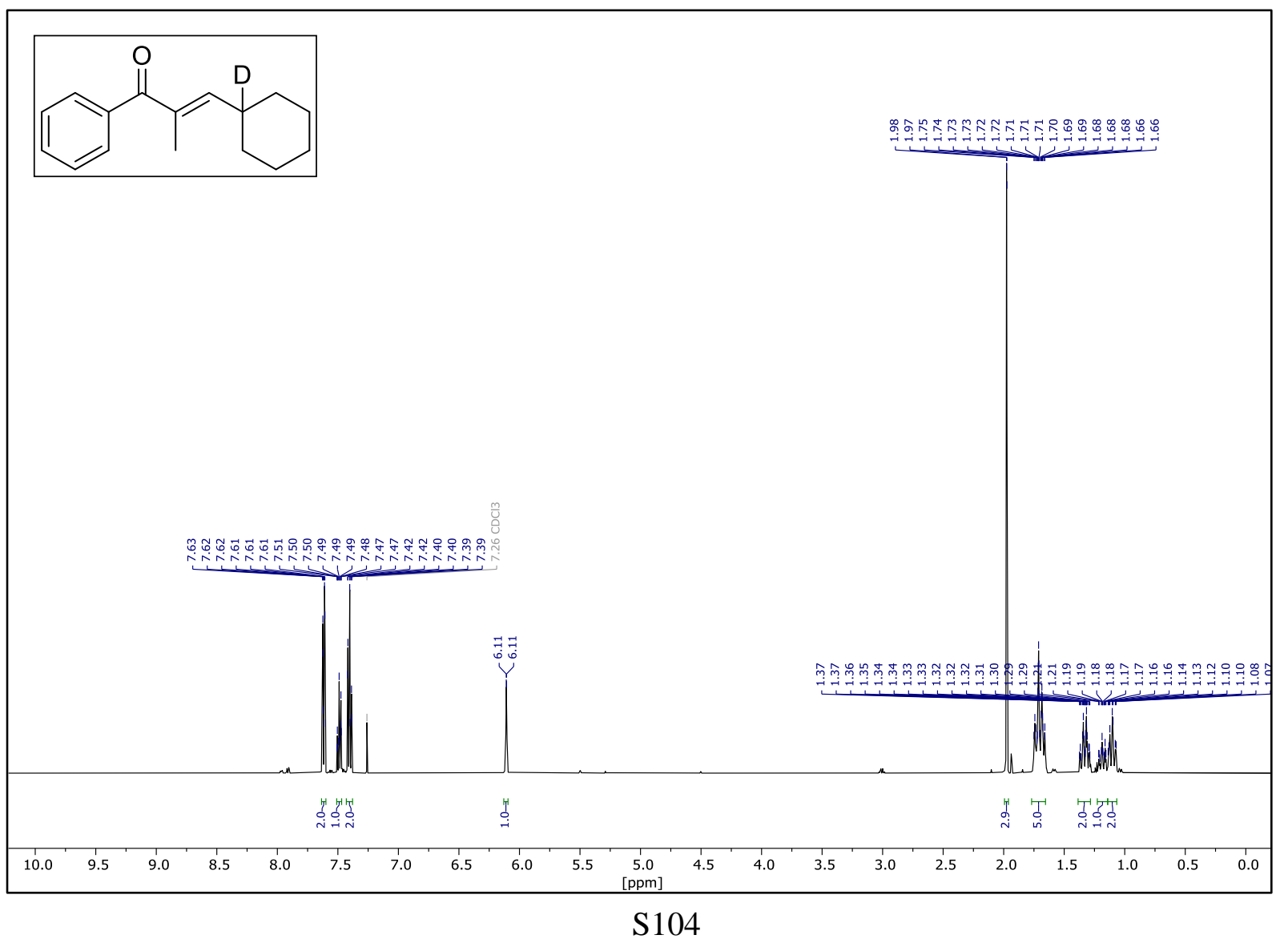


${ }^{13} \mathrm{C}$ NMR (126 MHz, $\left.\mathrm{CDCl}_{3}\right): d-\mathbf{2 2}$

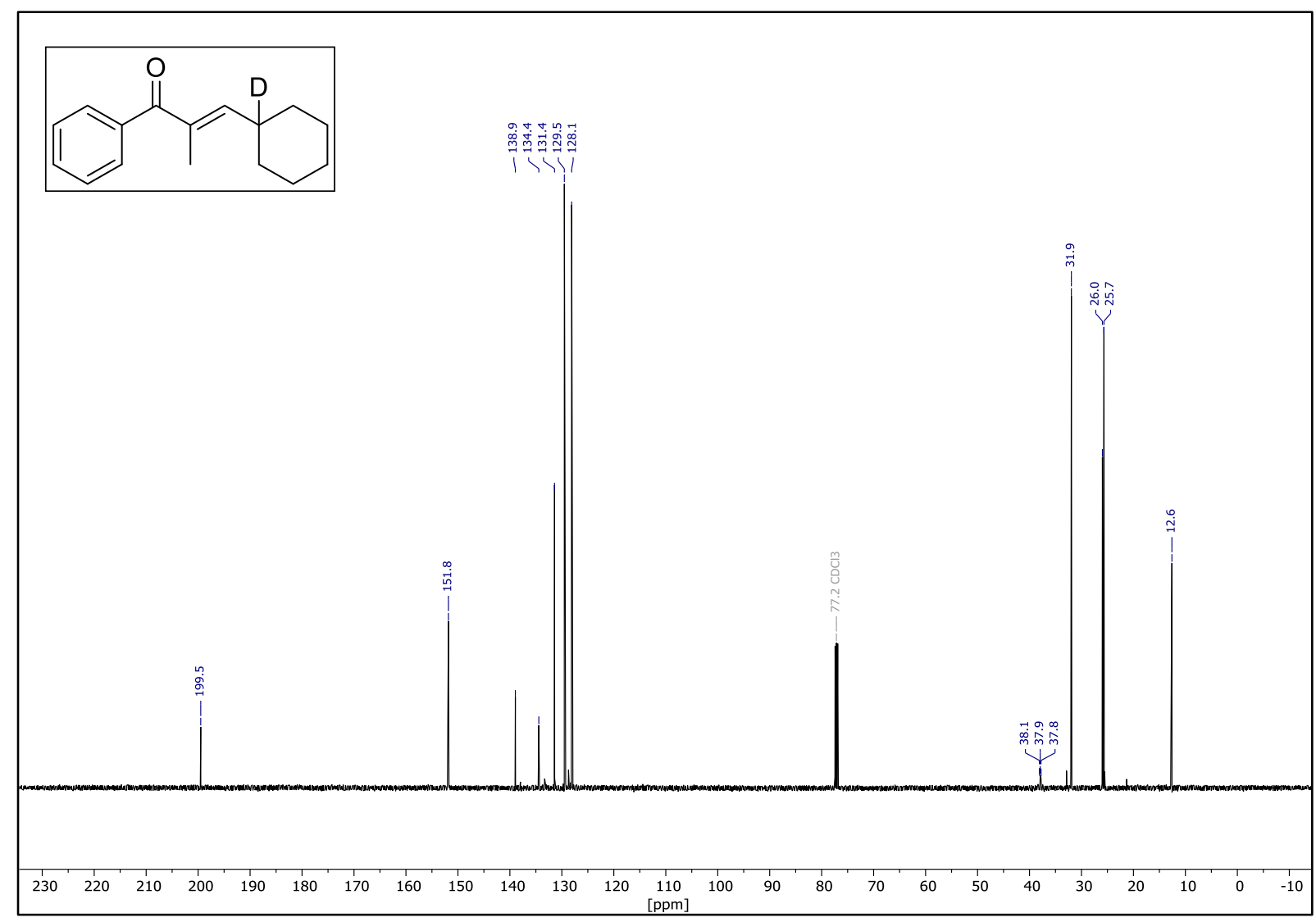

${ }^{1} \mathrm{H}$ NMR (400 MHz, $\mathrm{CDCl}_{3}$ ): cat. 9

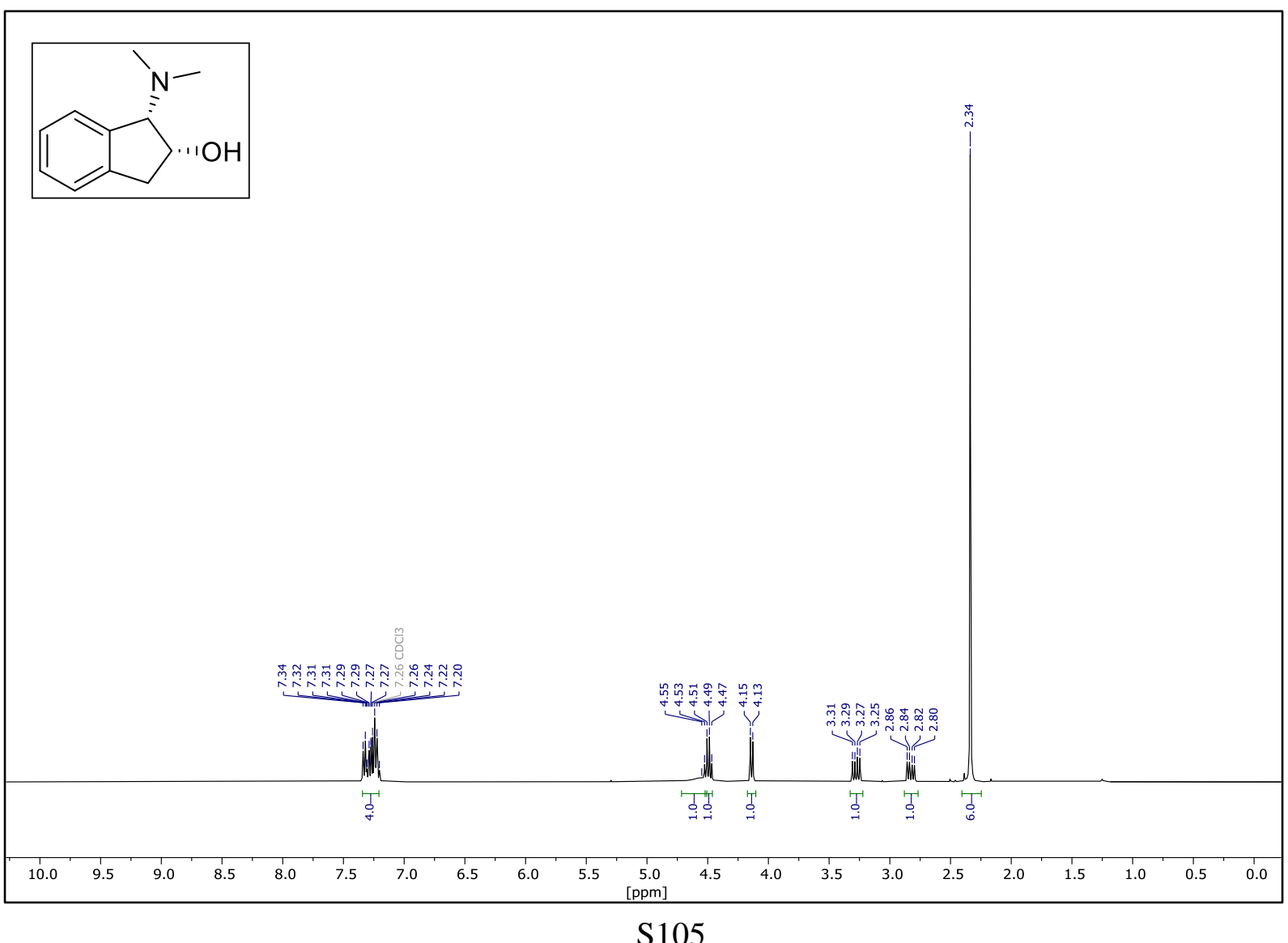


${ }^{1} \mathrm{H}$ NMR (400 MHz, $\left.\mathrm{CDCl}_{3}\right)$ : ( \pm )-2

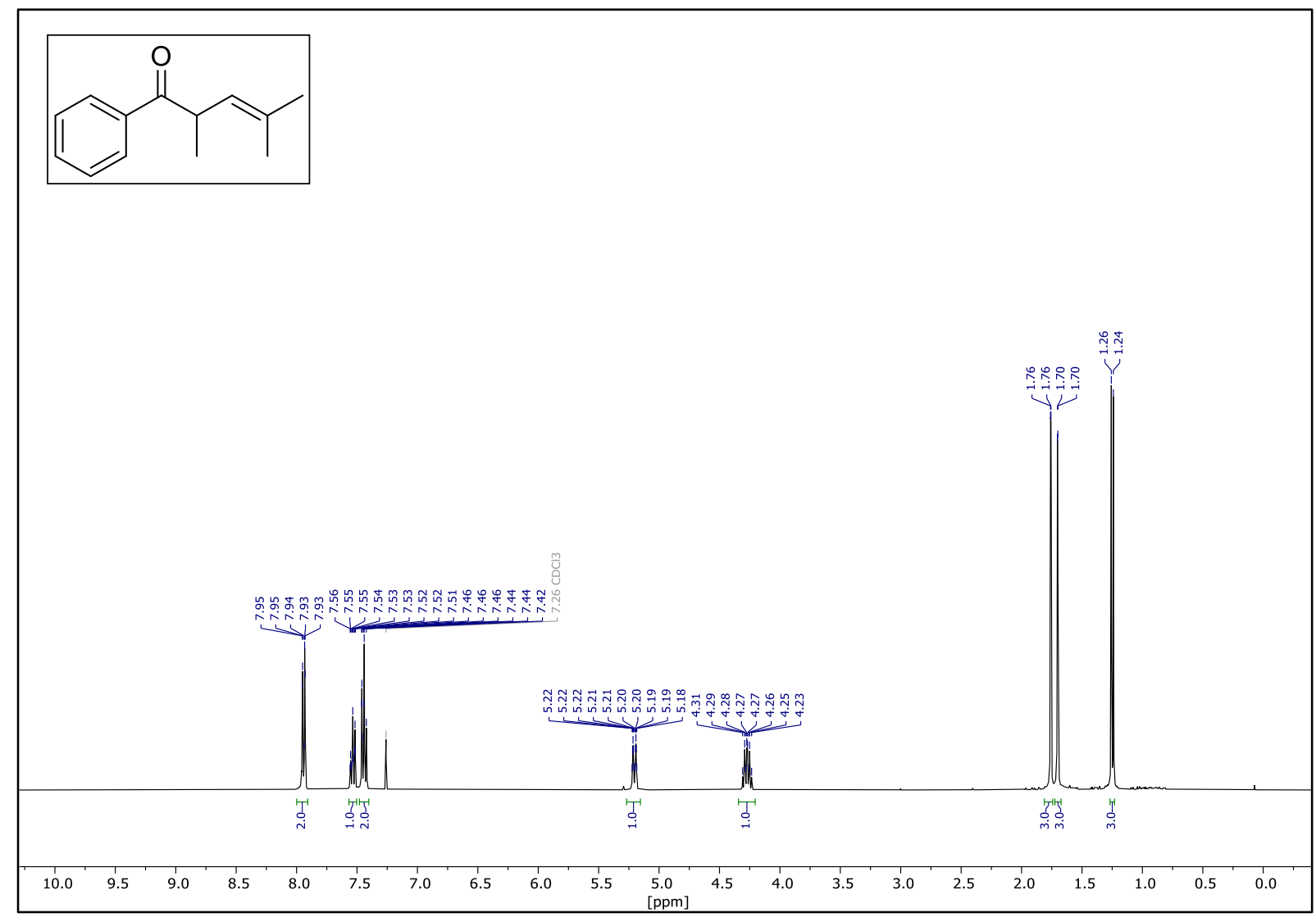

${ }^{1} \mathrm{H}$ NMR (400 MHz, $\left.\mathrm{CDCl}_{3}\right):( \pm)-3$

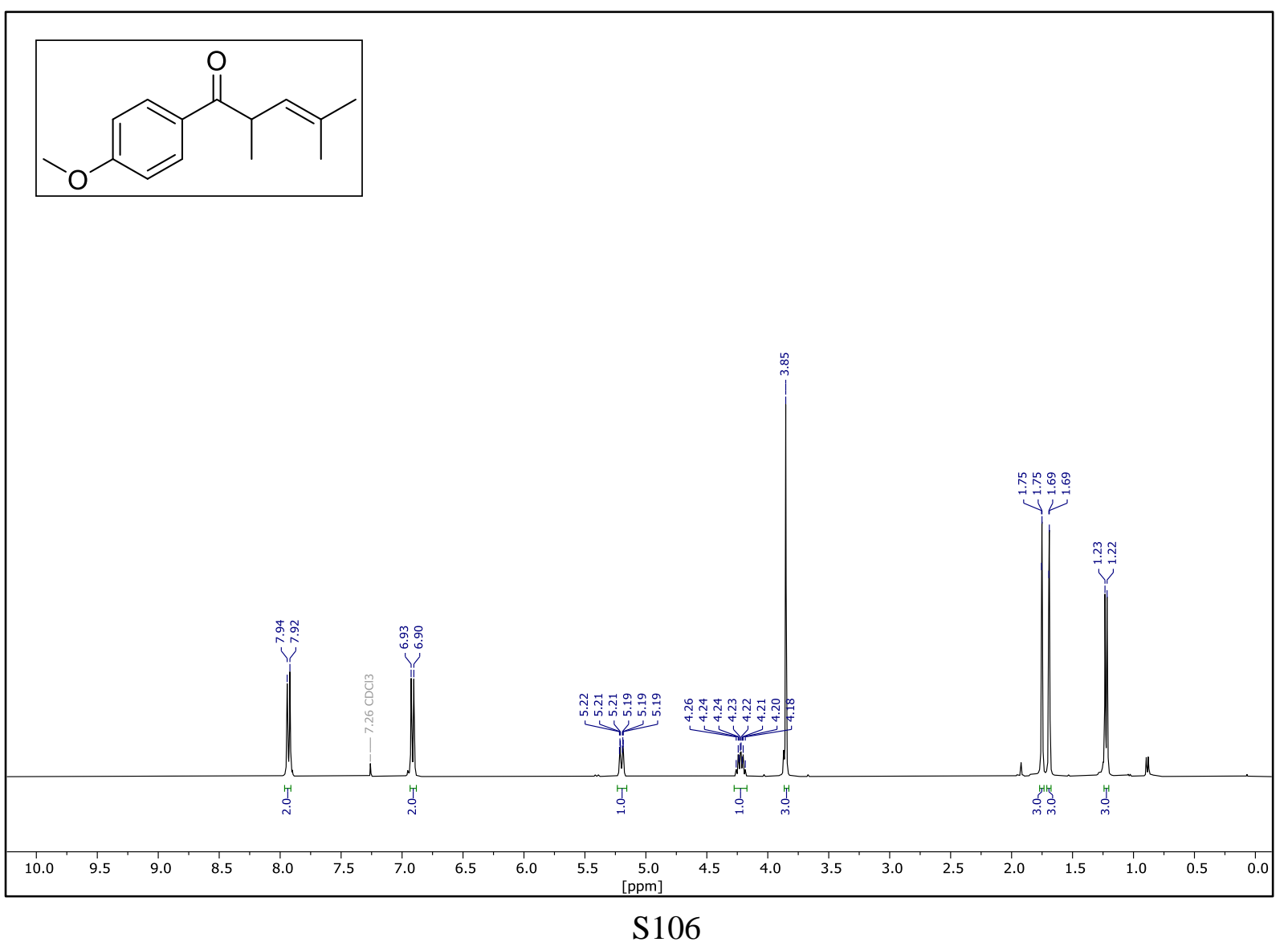


${ }^{1} \mathrm{H}$ NMR (600 MHz, $\left.\mathrm{CDCl}_{3}\right):( \pm)-4$

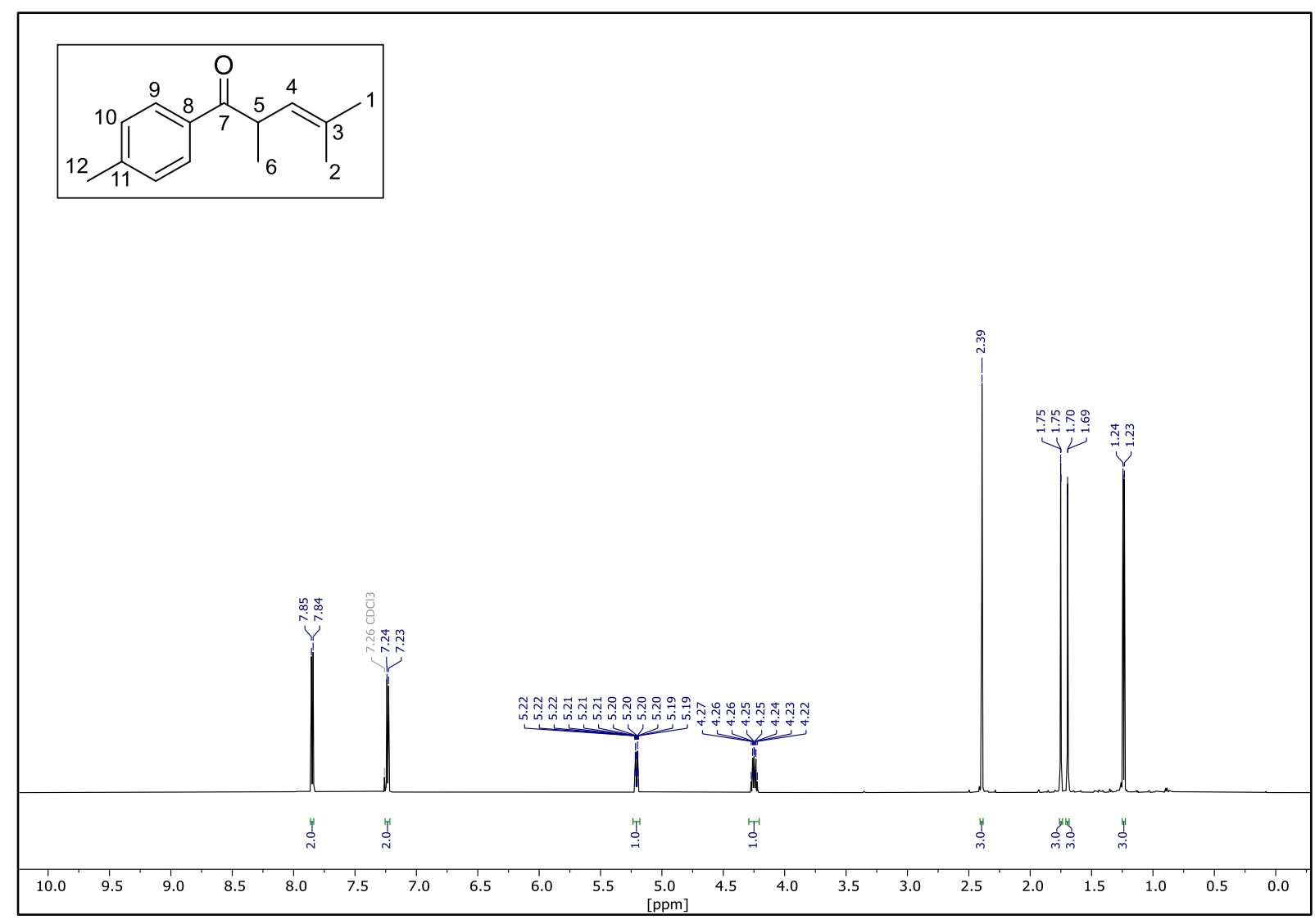

${ }^{13} \mathrm{C}$ NMR (151 MHz, $\left.\mathrm{CDCl}_{3}\right)$ : ( \pm )-4

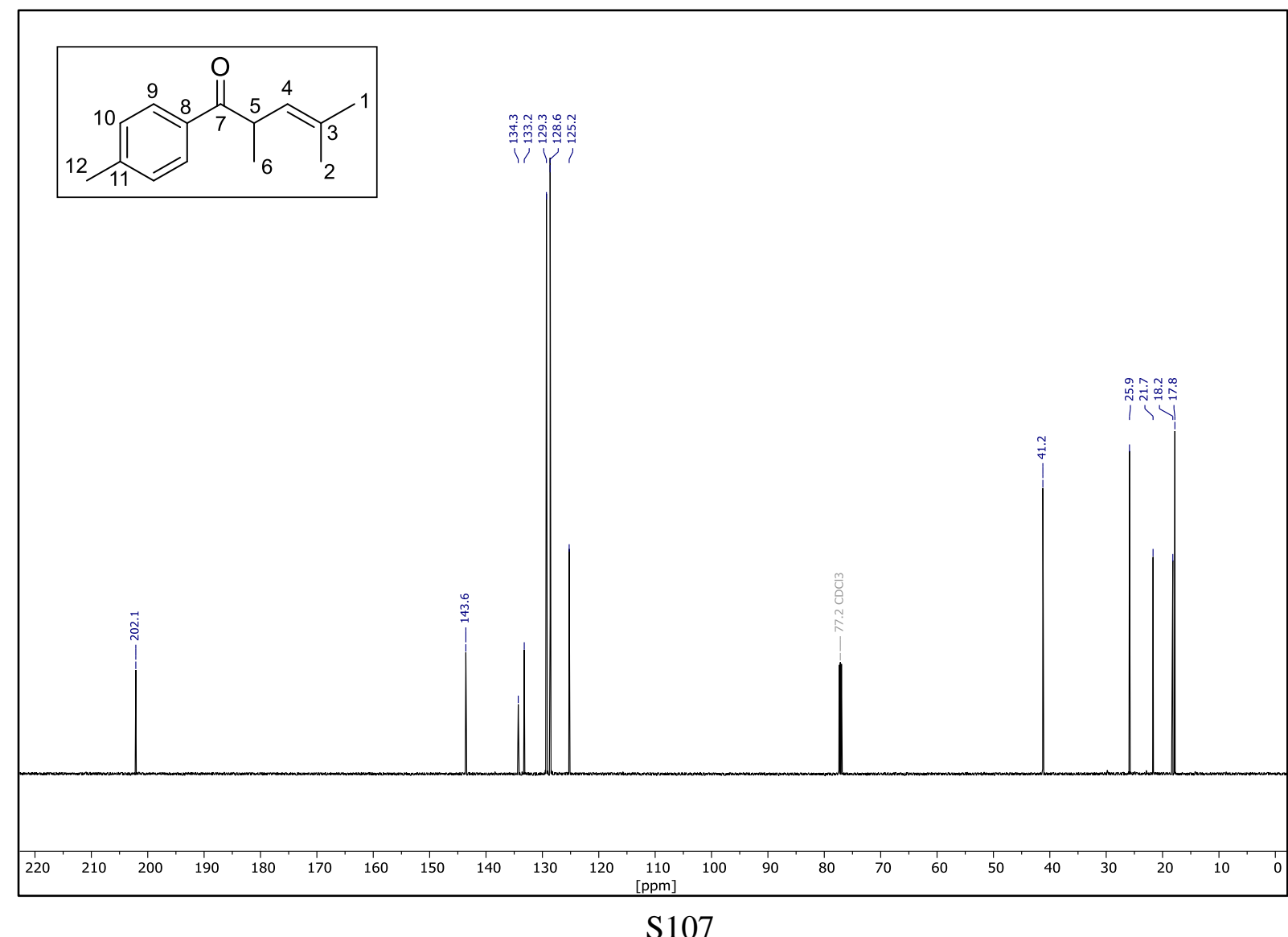


${ }^{1} \mathrm{H}$ NMR (500 MHz, $\left.\mathrm{CDCl}_{3}\right):( \pm)-5$

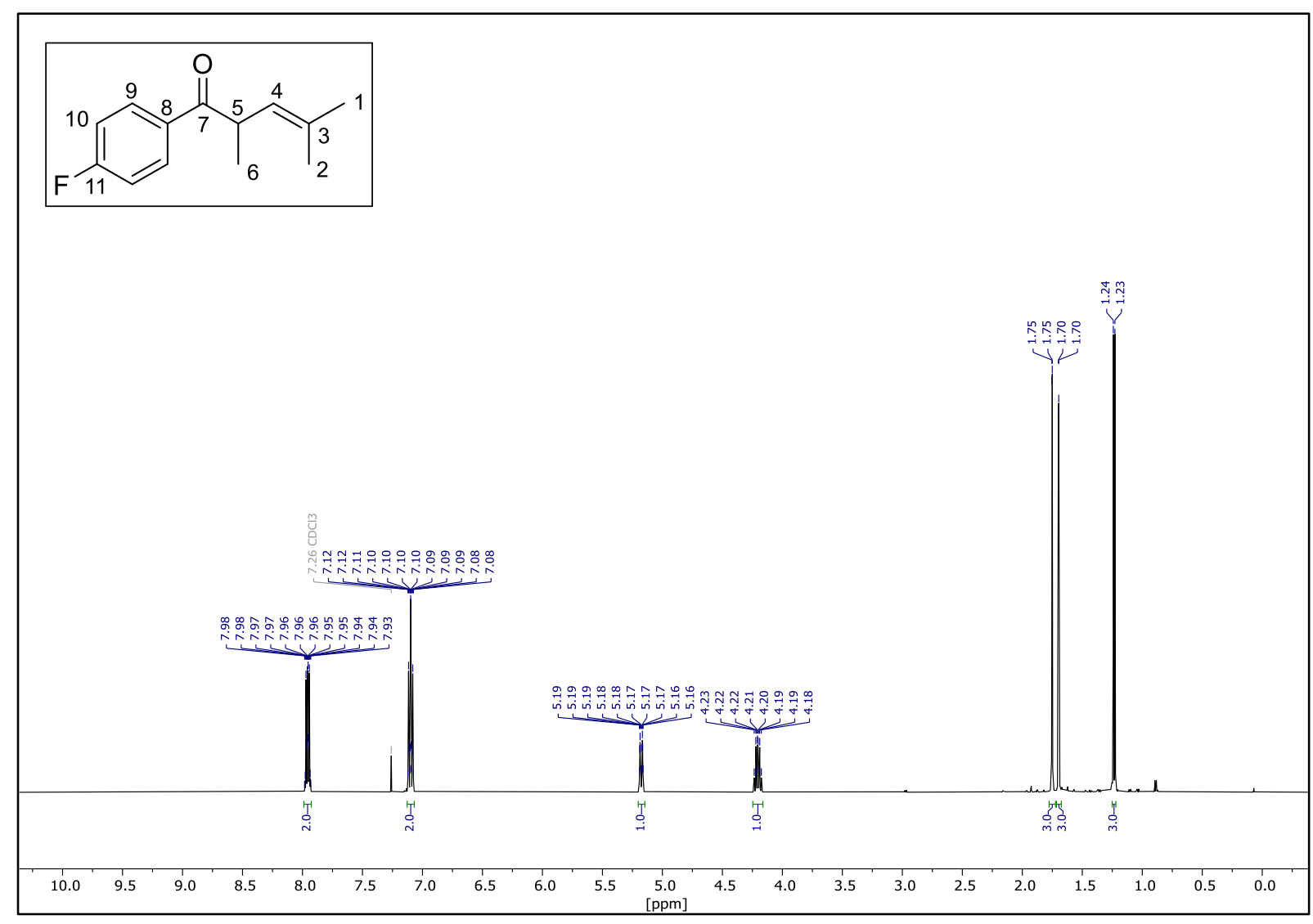

${ }^{13} \mathrm{C}$ NMR (126 MHz, $\left.\mathrm{CDCl}_{3}\right):( \pm)-5$
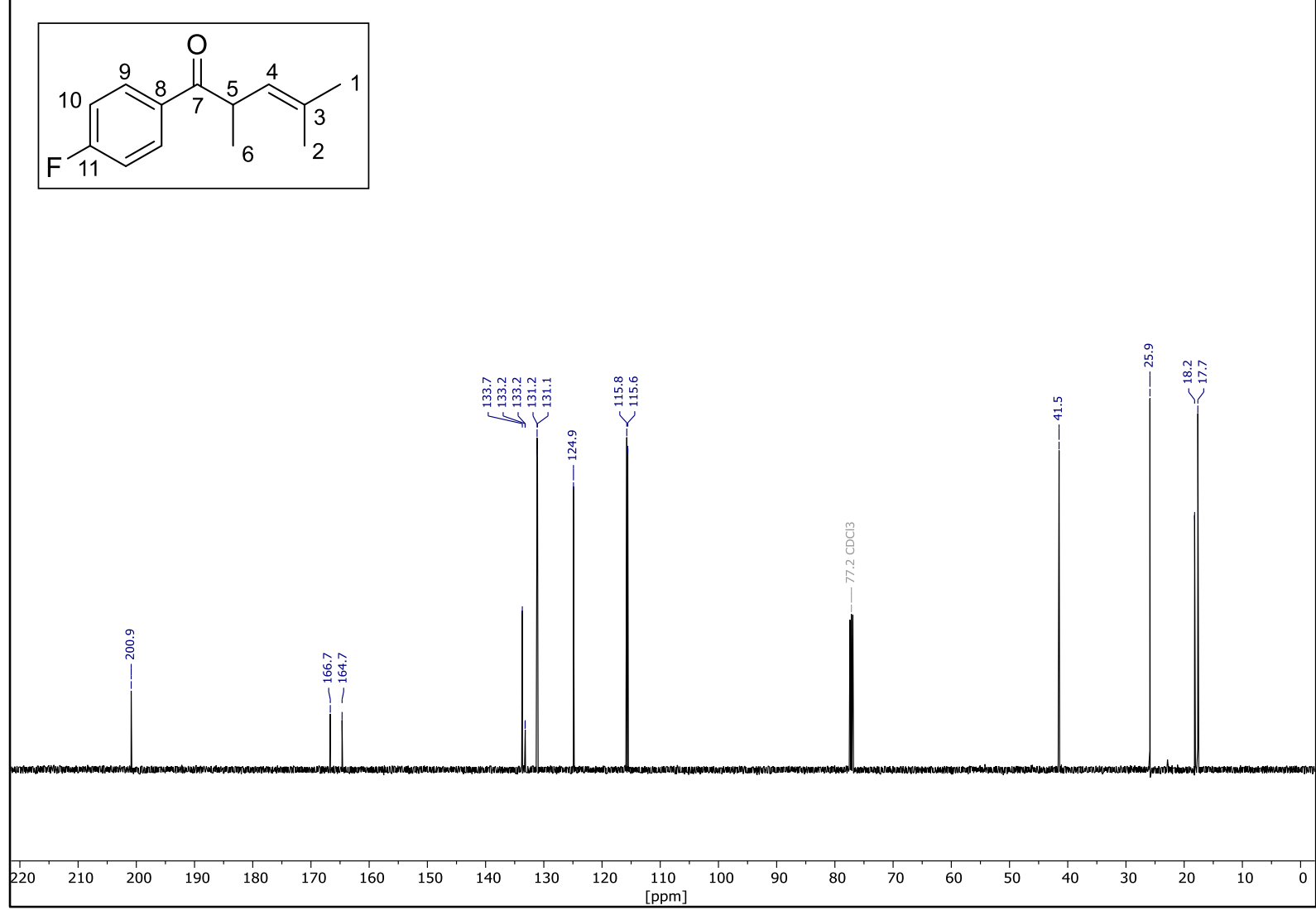
${ }^{19}$ F NMR (470 MHz, $\left.\mathrm{CDCl}_{3}\right):( \pm)-5$

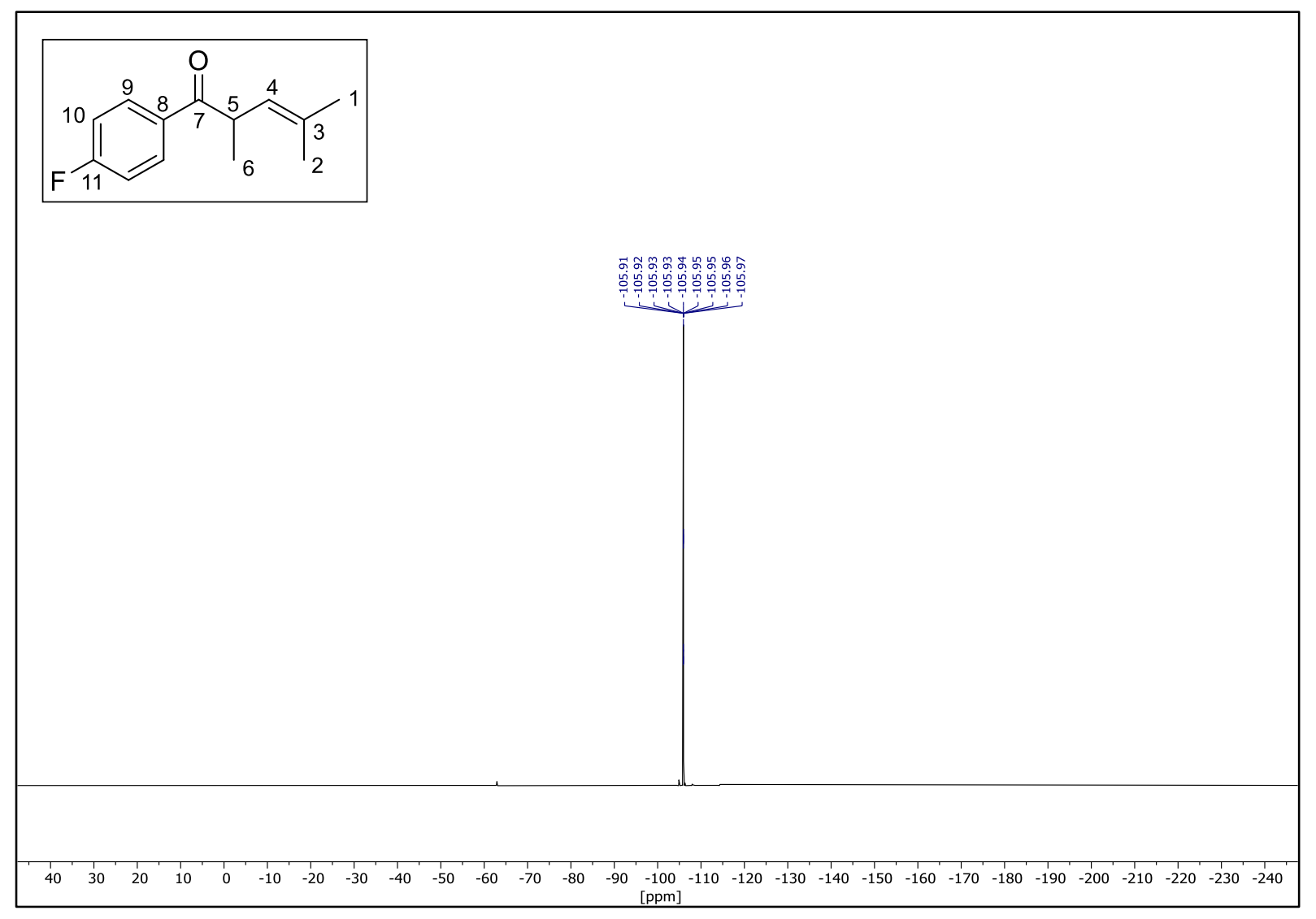

${ }^{1} \mathrm{H} \mathrm{NMR}\left(500 \mathrm{MHz}, \mathrm{CDCl}_{3}\right)$ : ( \pm )-6

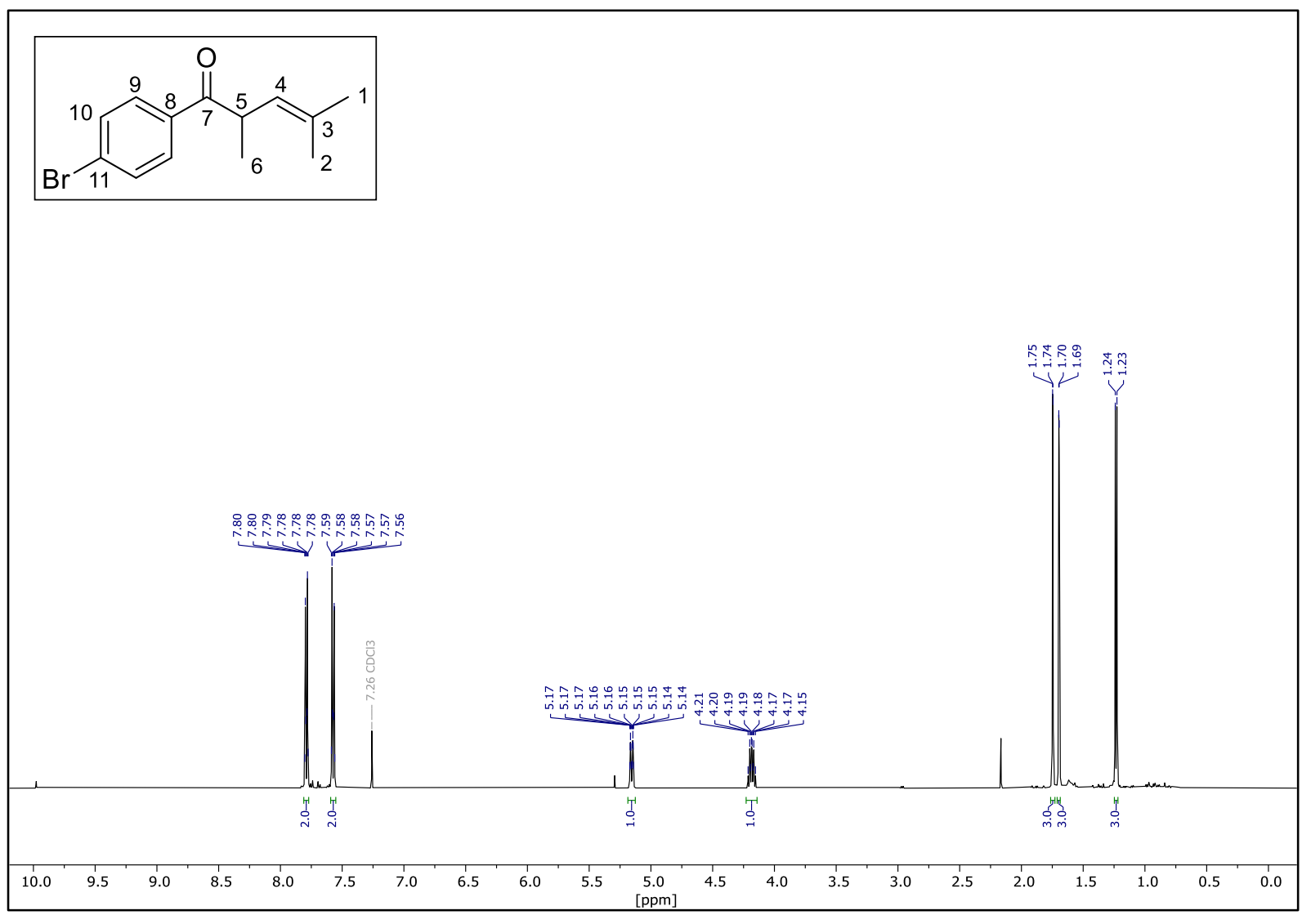


${ }^{13} \mathrm{C}$ NMR (126 MHz, $\left.\mathrm{CDCl}_{3}\right):( \pm)-6$

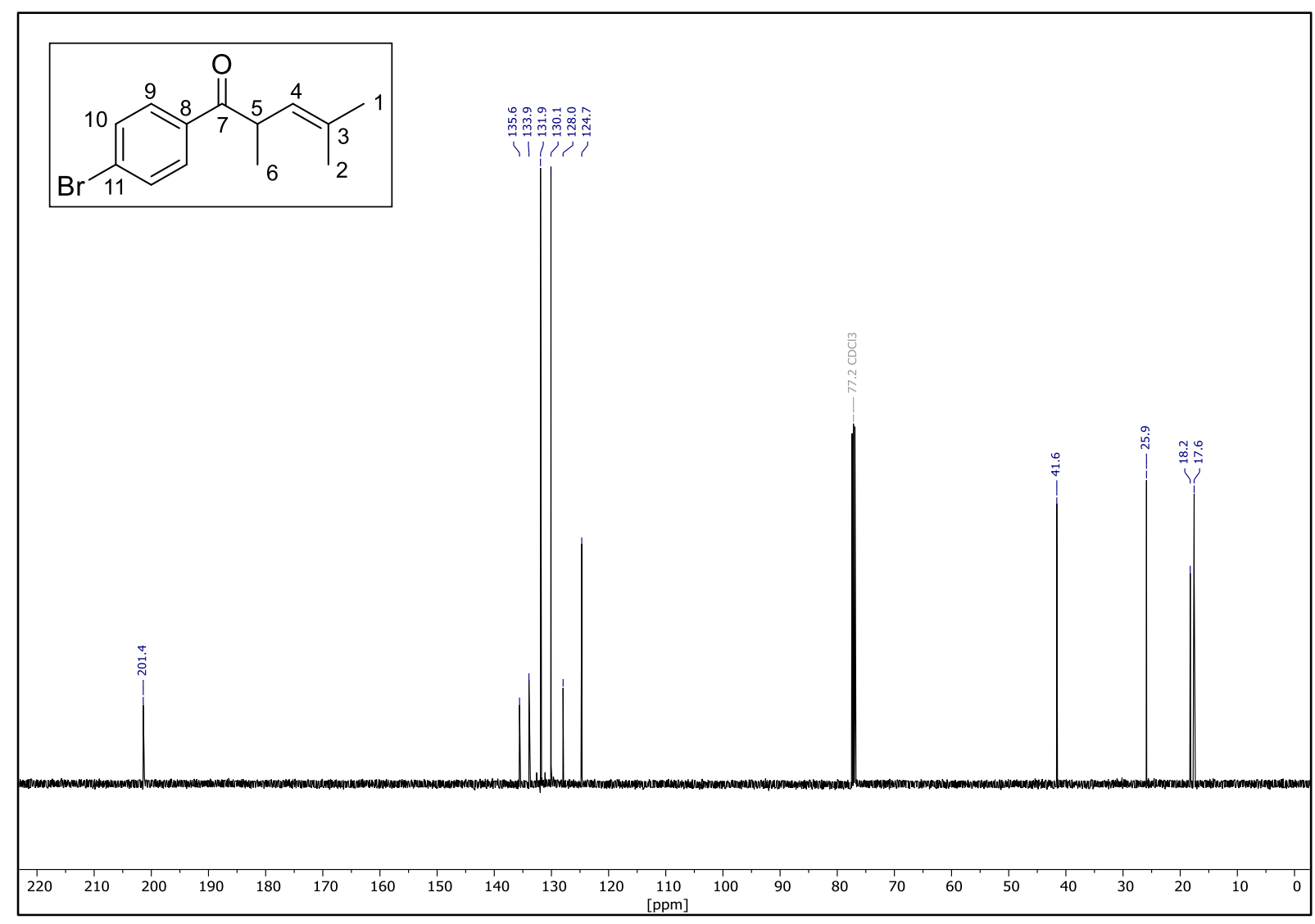

${ }^{1} \mathrm{H}$ NMR (600 MHz, $\left.\mathrm{CDCl}_{3}\right)$ : ( \pm )-7

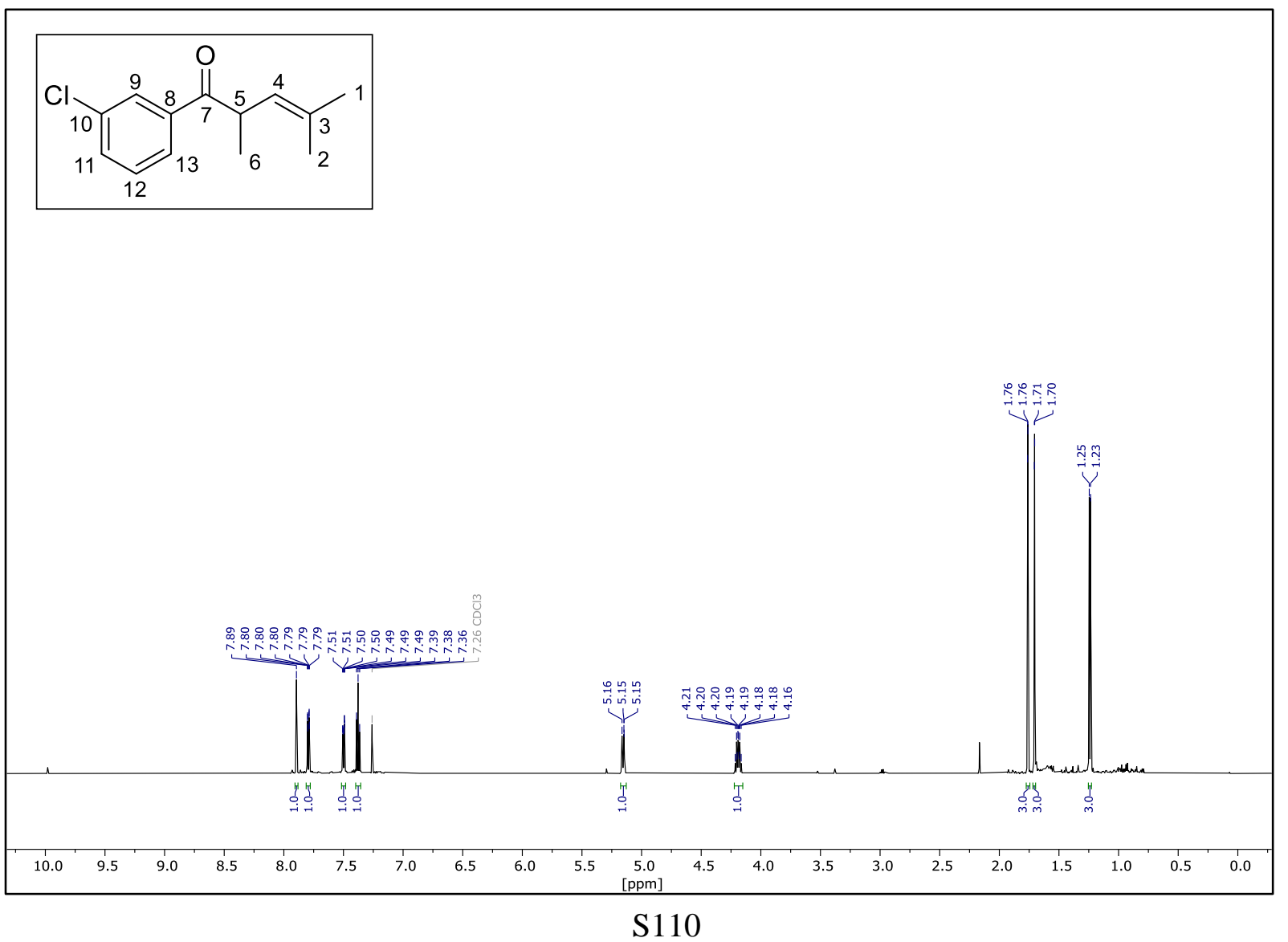


${ }^{13} \mathrm{C}$ NMR (151 MHz, $\left.\mathrm{CDCl}_{3}\right):( \pm)-7$

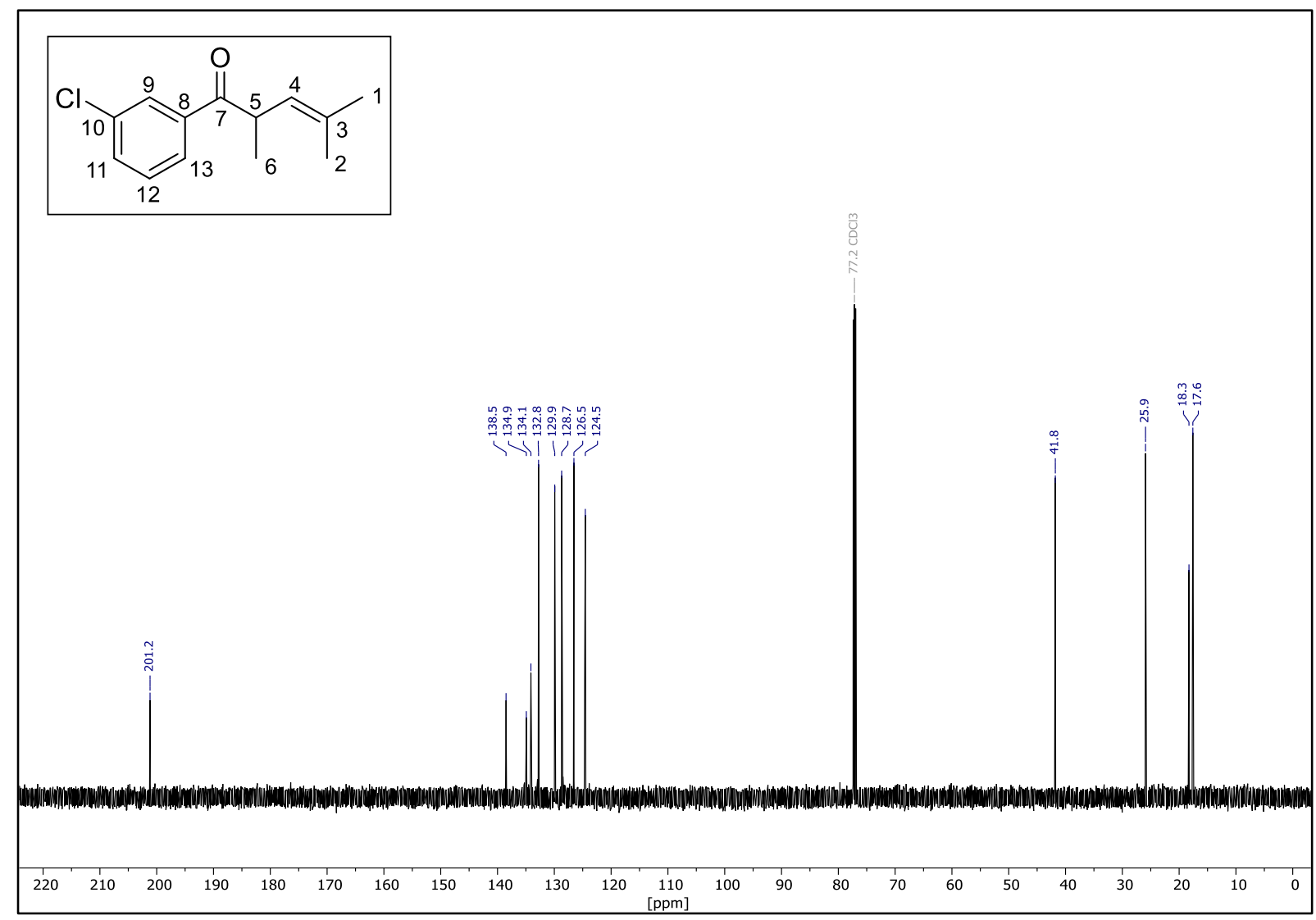

${ }^{1} \mathrm{H}$ NMR (500 MHz, $\left.\mathrm{CDCl}_{3}\right):( \pm)-(Z)-8$

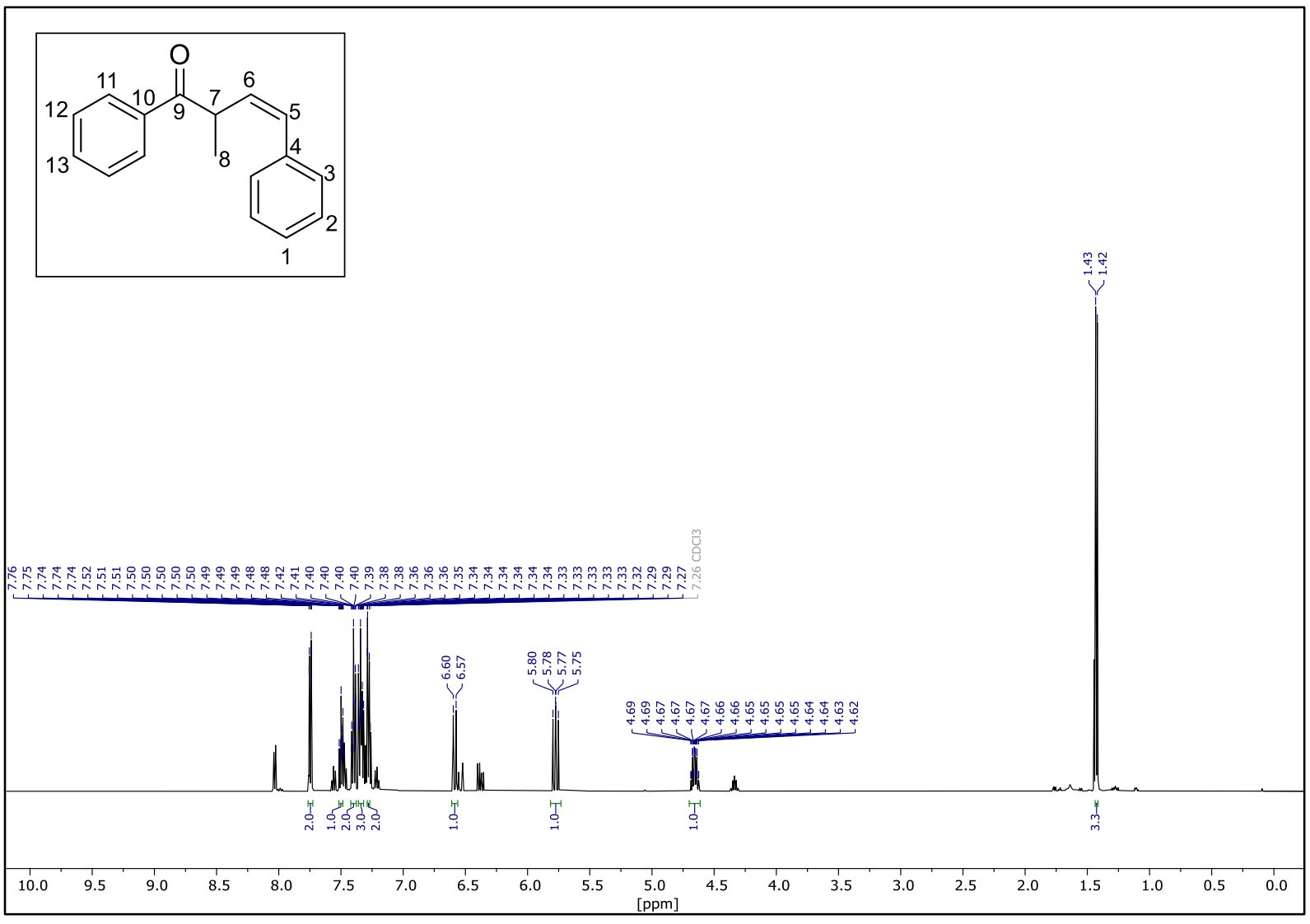


${ }^{1} \mathrm{H}$ NMR (500 MHz, $\left.\mathrm{CDCl}_{3}\right)$ : ( \pm )-(E)-8

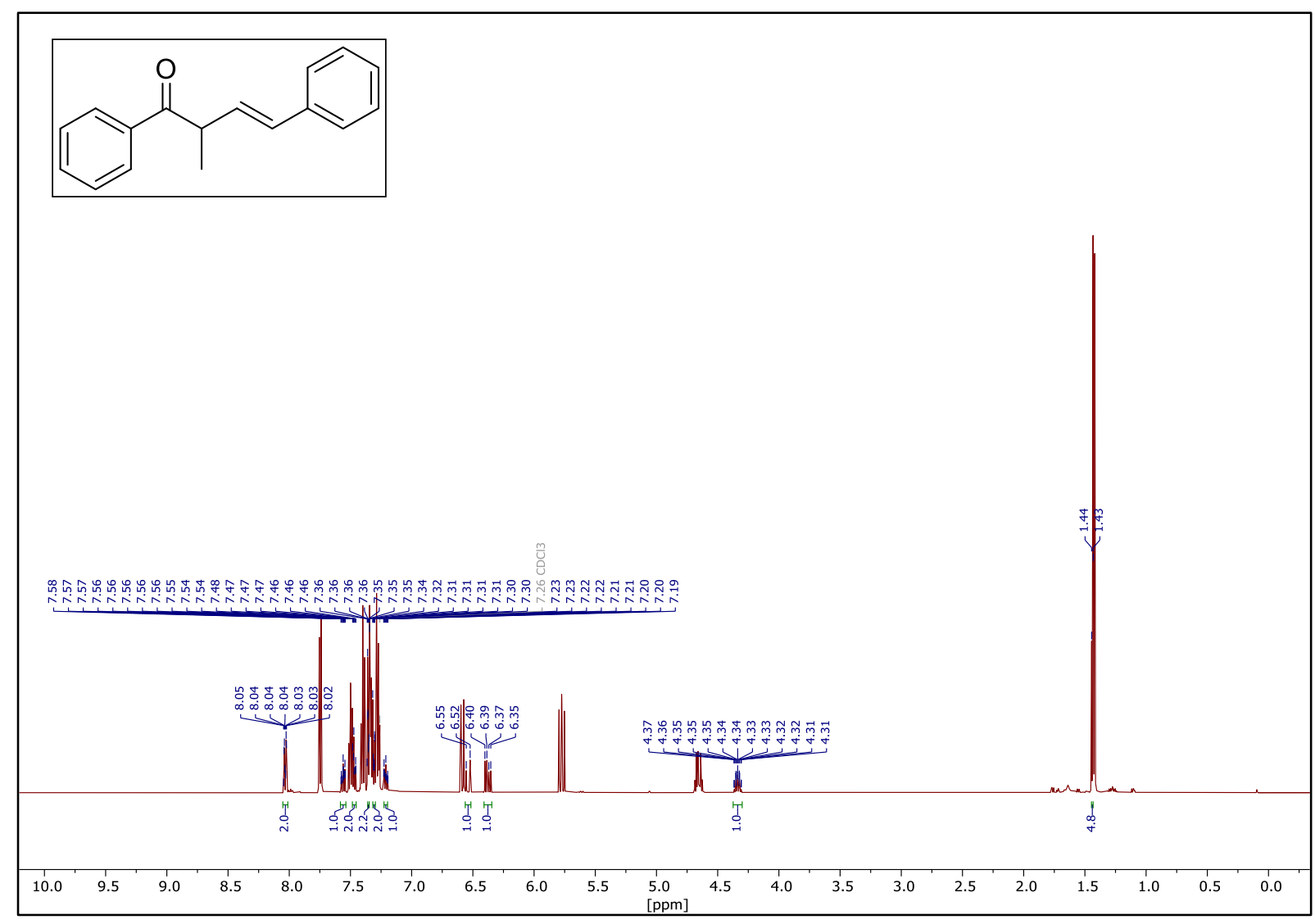

${ }^{13} \mathrm{C}$ NMR (126 MHz, $\left.\mathrm{CDCl}_{3}\right)$ : ( \pm )-(Z)-8

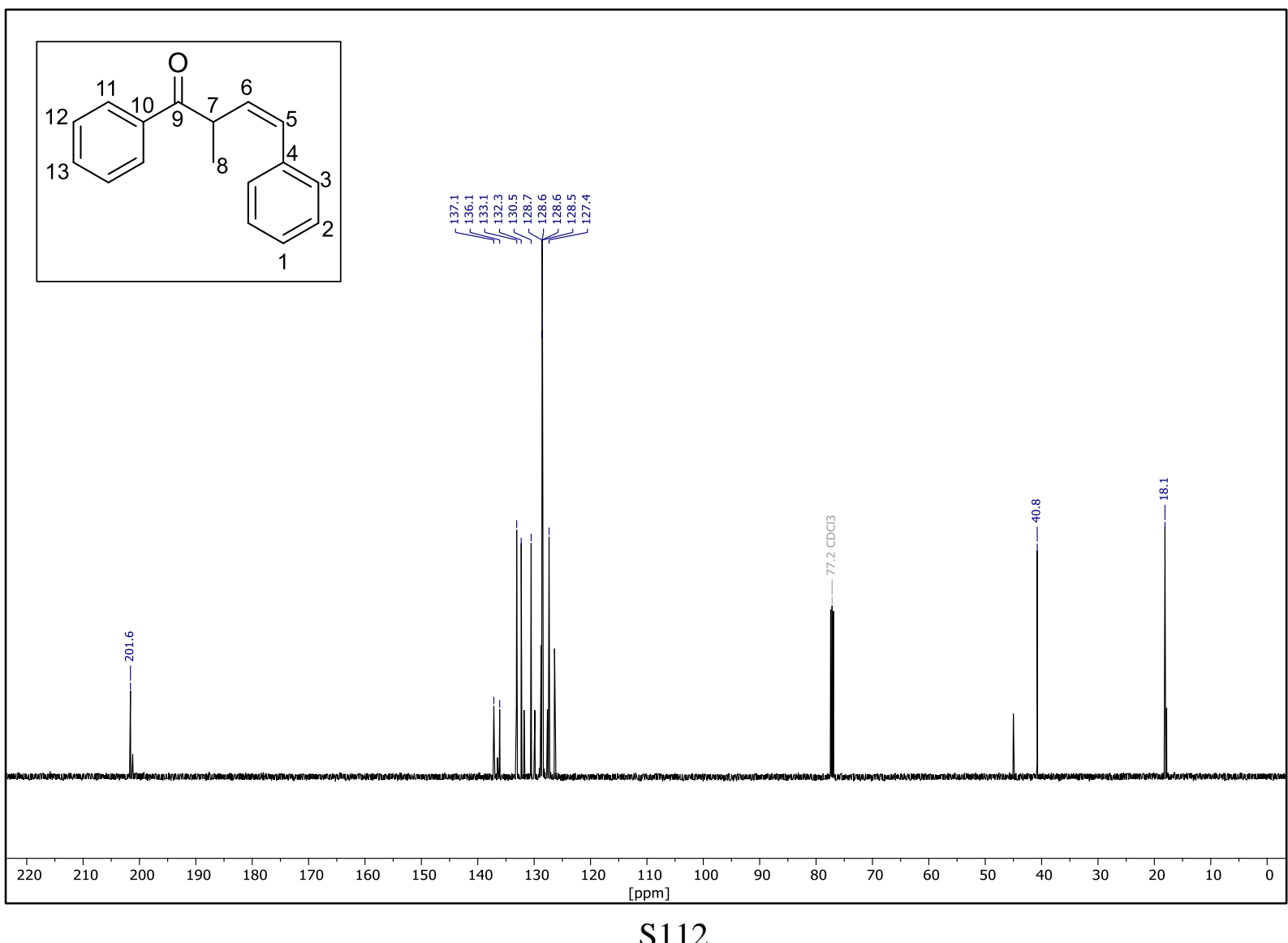


${ }^{1} \mathrm{H},{ }^{1} \mathrm{H} \operatorname{COSY}\left(500 \mathrm{MHz}, \mathrm{CDCl}_{3}\right):( \pm)-(\mathrm{Z} / \mathrm{E})-\mathbf{8}$

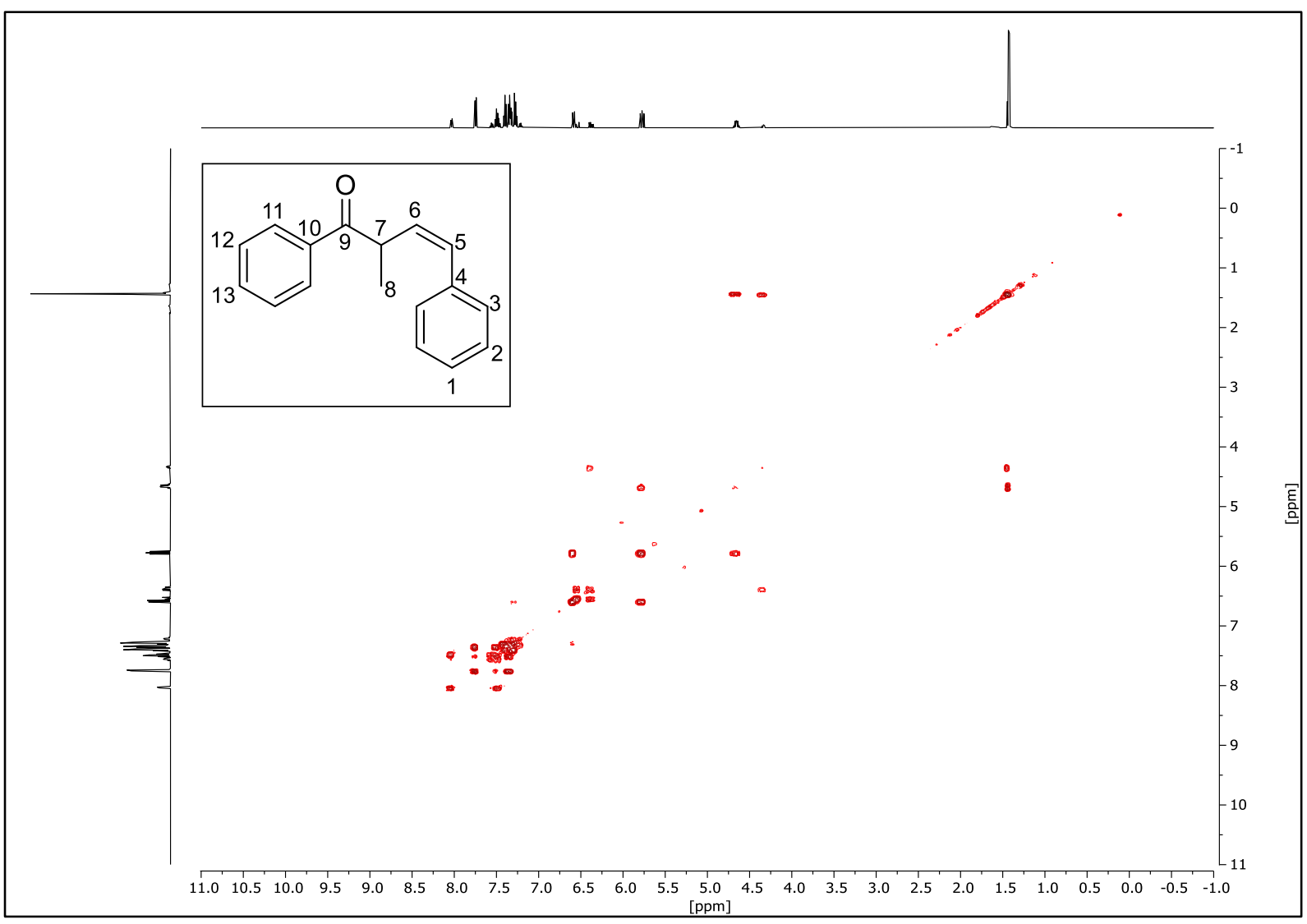

${ }^{1} \mathrm{H},{ }^{13} \mathrm{C}$ HMBC $\left(500 \mathrm{MHz}, \mathrm{CDCl}_{3}\right)$ : ( \pm )-(Z/E)-8

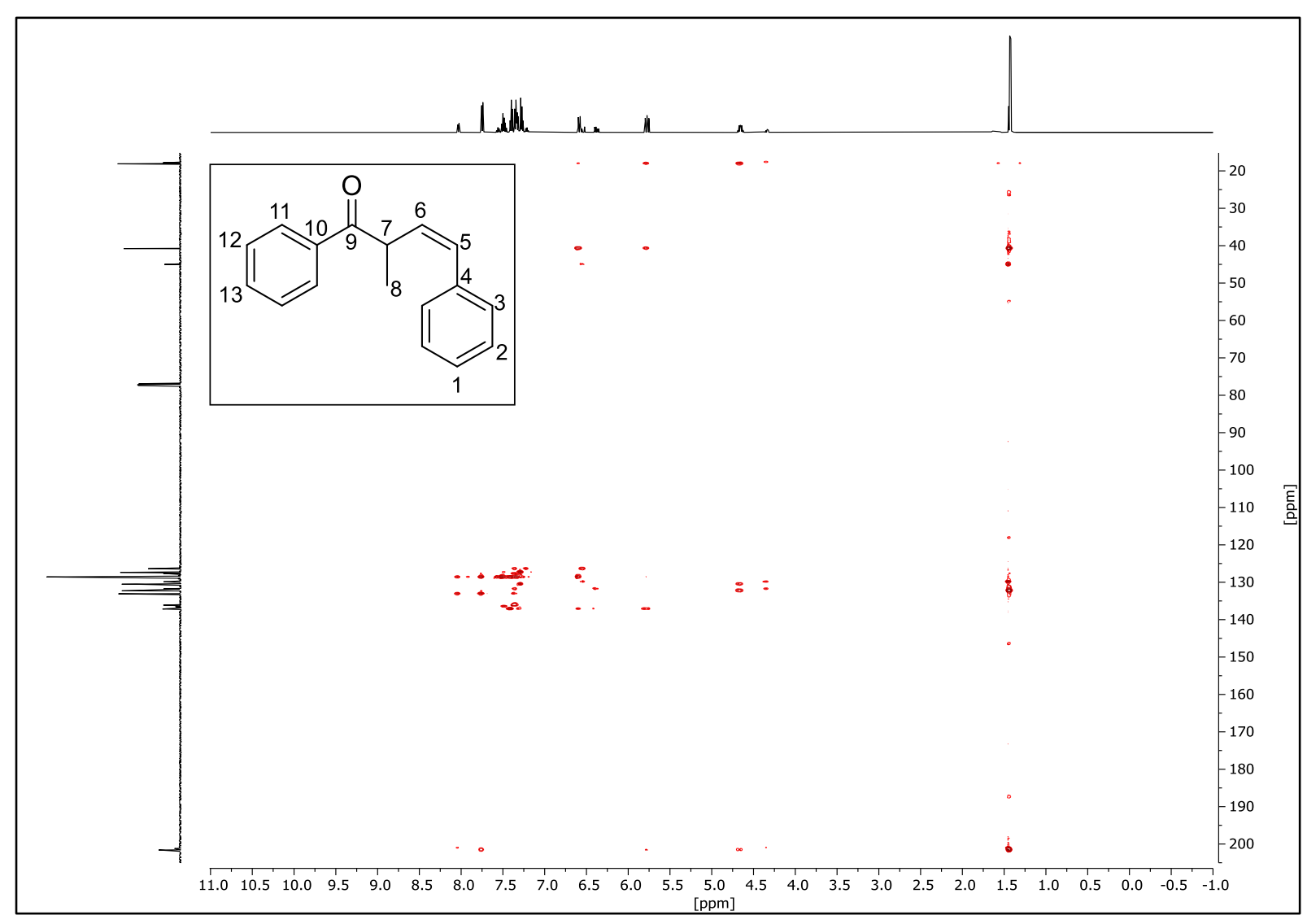


${ }^{1} \mathrm{H},{ }^{13} \mathrm{C}$ HSQC (500 MHz, CDCl $):( \pm)-(\mathrm{Z} / \mathrm{E})-\mathbf{8}$

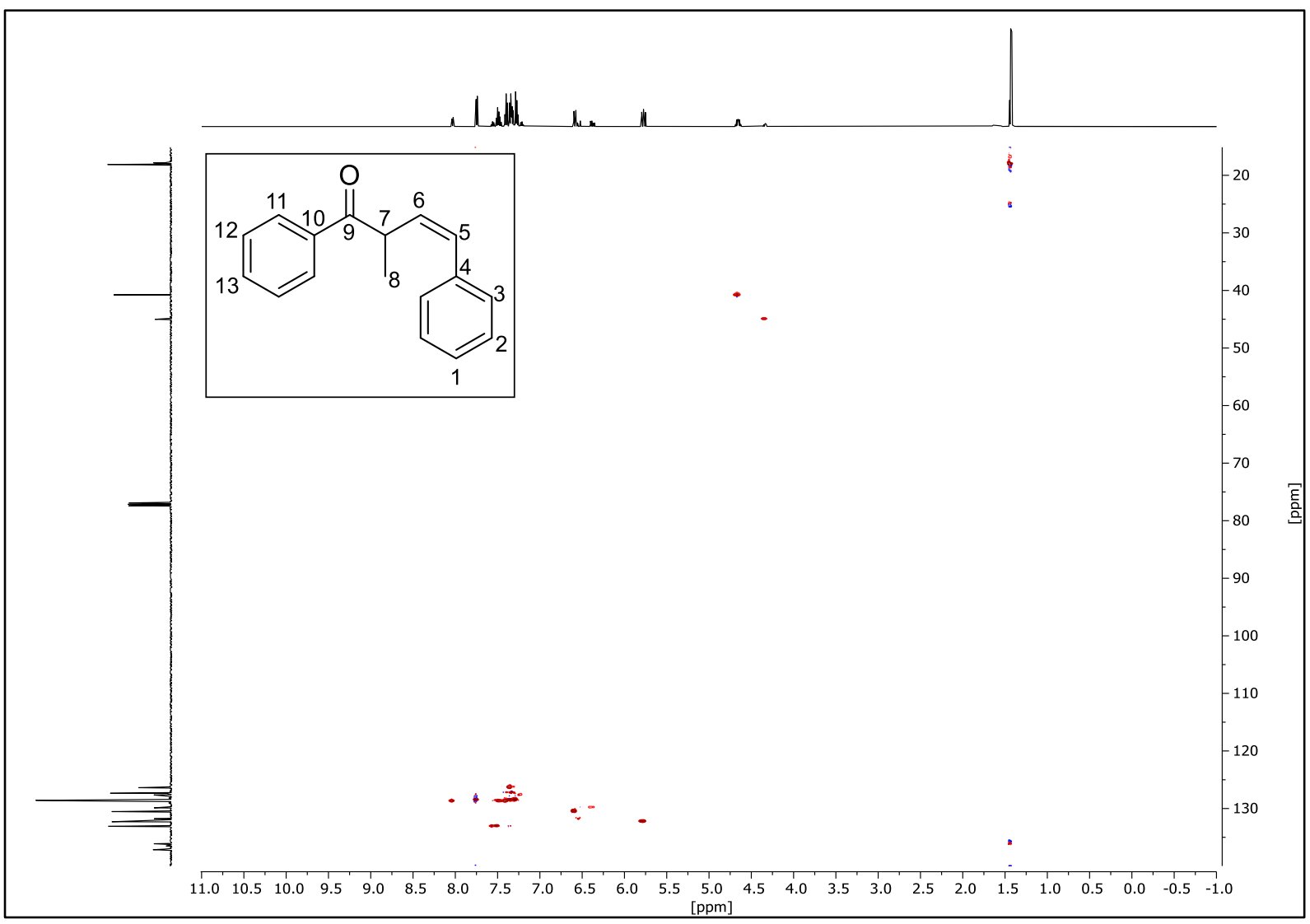

1D NOESY (500 MHz, CDCl 3 ): ( \pm )-(Z/E)-8

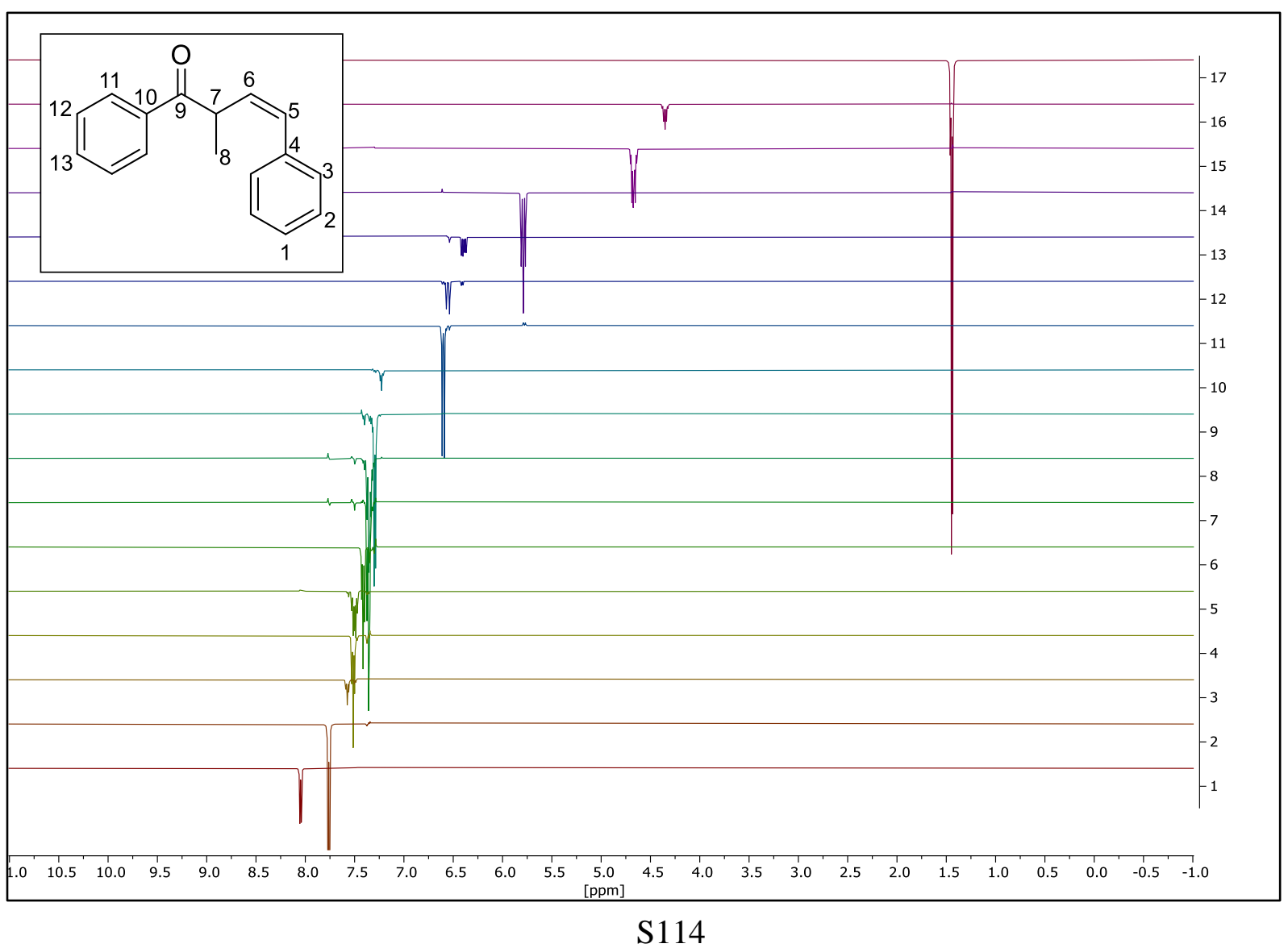


${ }^{1} \mathrm{H}$ NMR (500 MHz, $\left.\mathrm{CDCl}_{3}\right)$ : ( \pm )-9

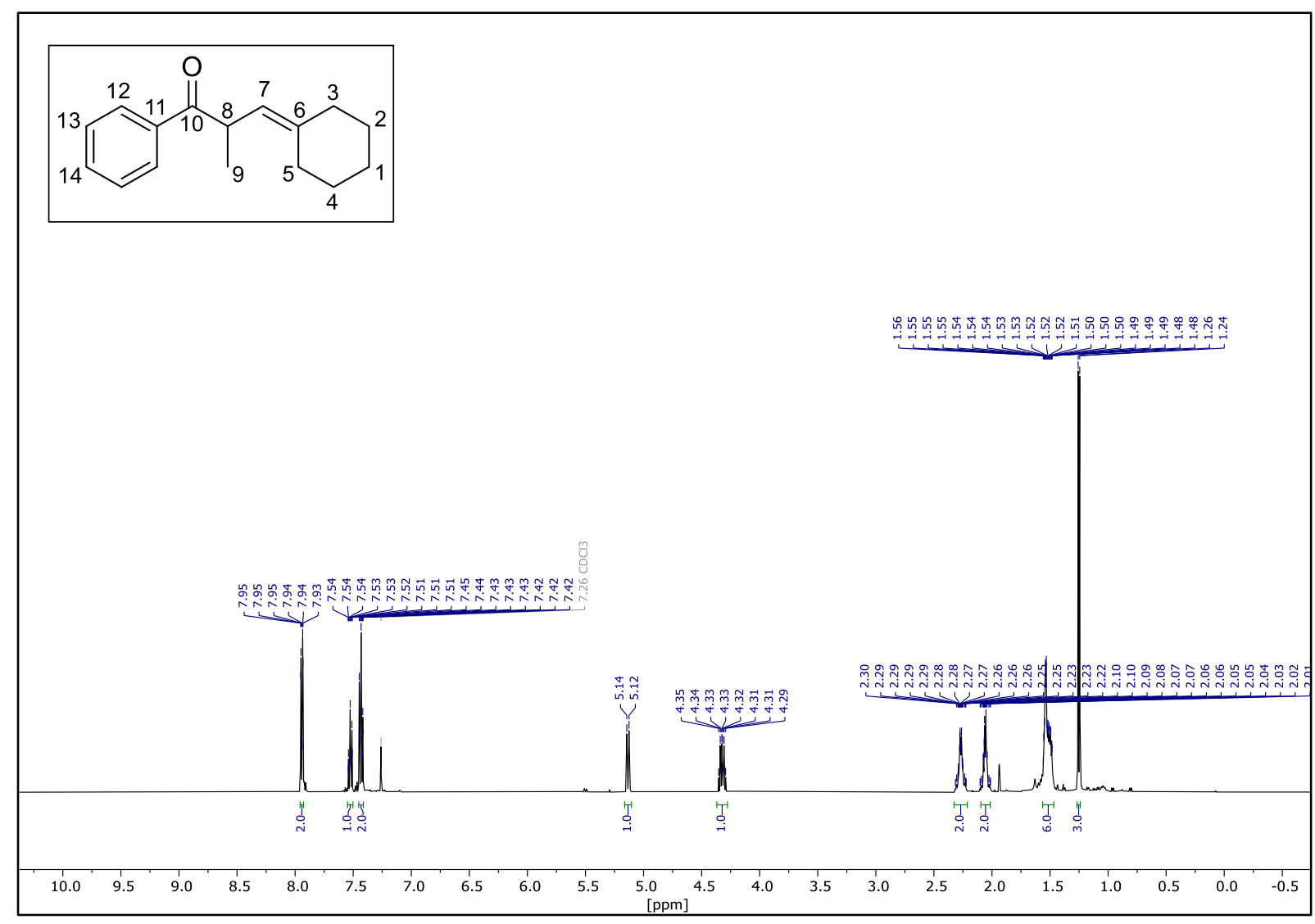

${ }^{13} \mathrm{C}$ NMR (126 MHz, $\left.\mathrm{CDCl}_{3}\right)$ : ( \pm )-9

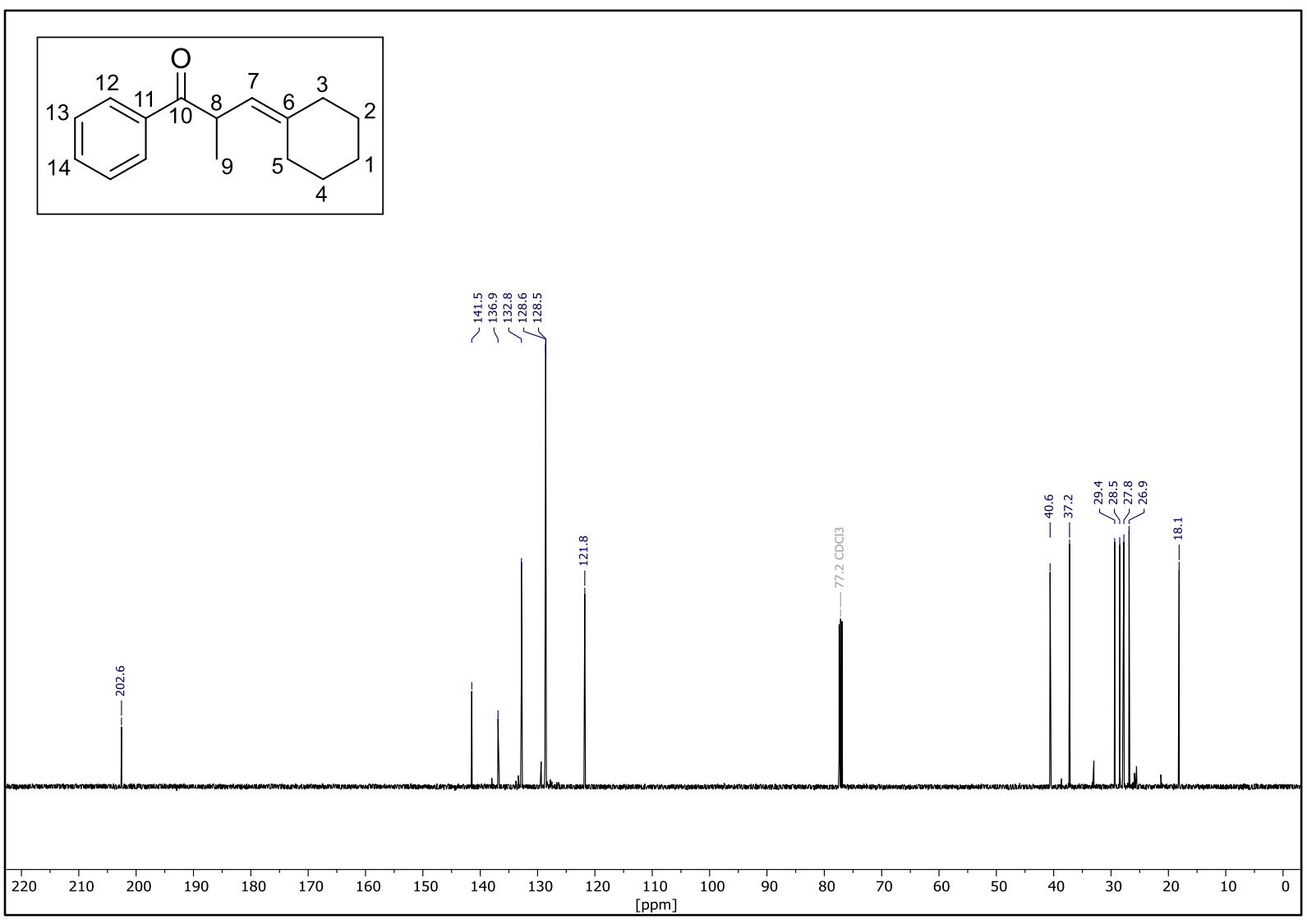


${ }^{1} \mathrm{H}$ NMR (500 MHz, $\left.\mathrm{CDCl}_{3}\right)$ : ( \pm )-10

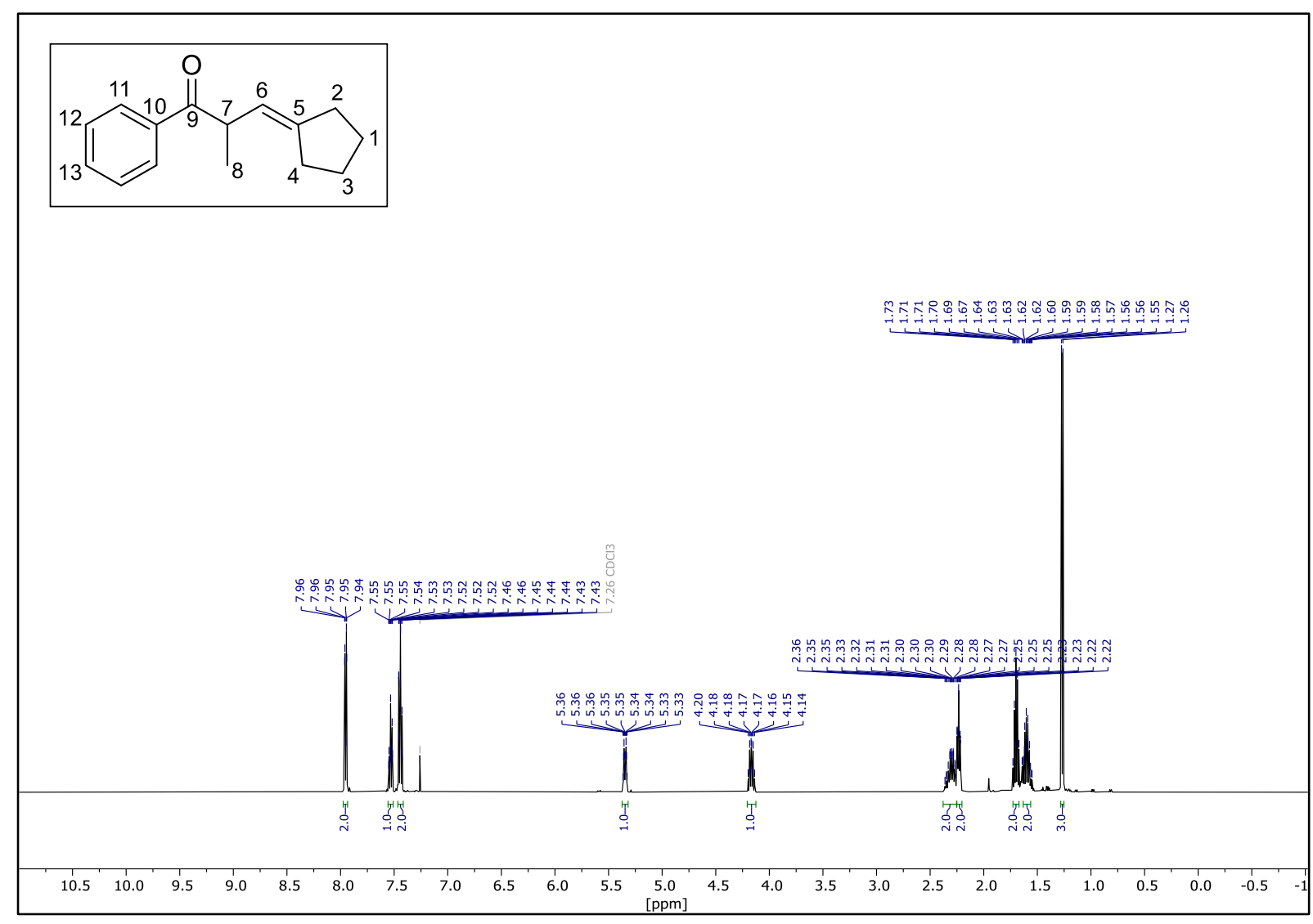

${ }^{13} \mathrm{C}$ NMR (126 MHz, $\left.\mathrm{CDCl}_{3}\right):( \pm)-10$

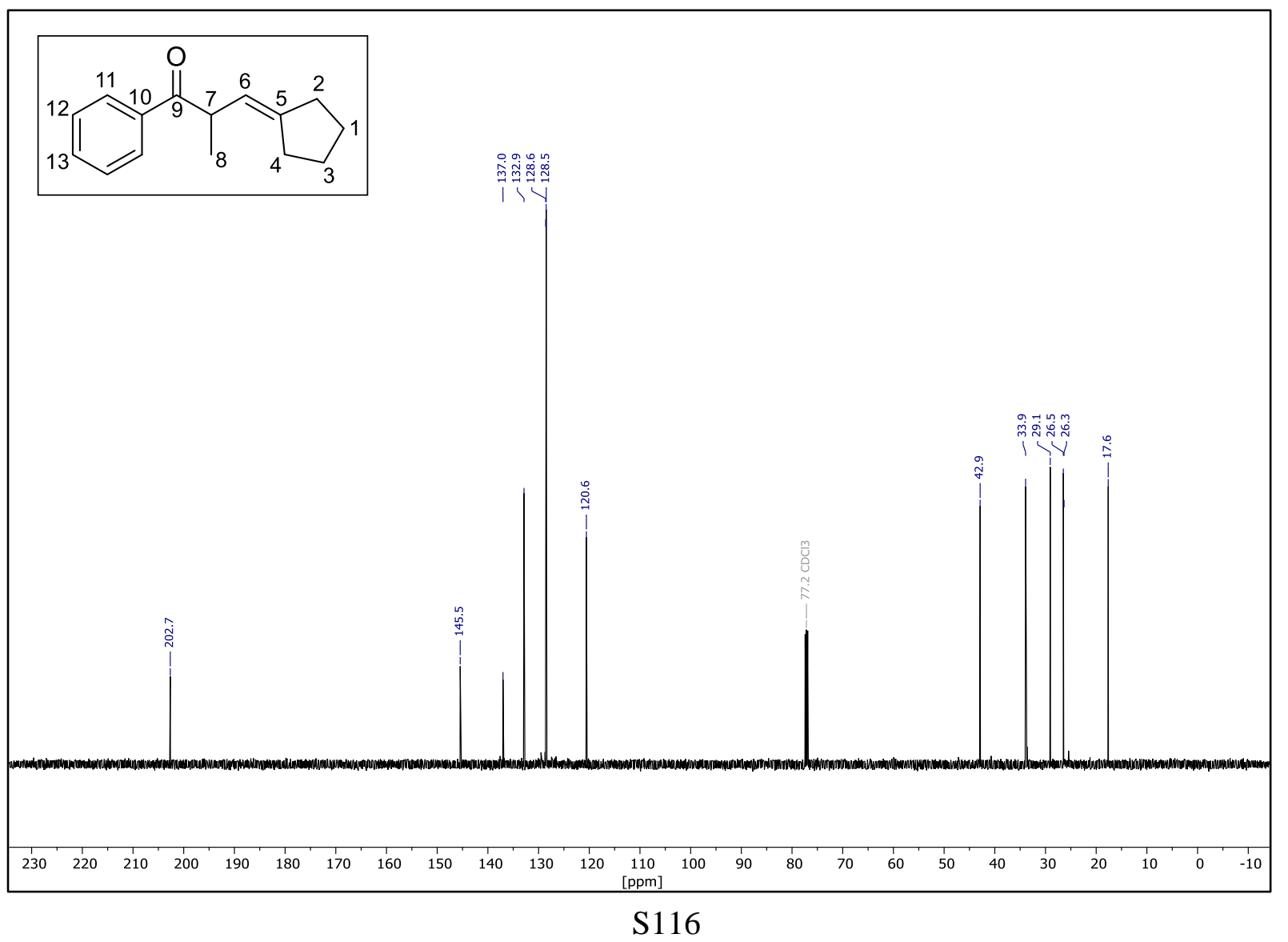


${ }^{1} \mathrm{H}$ NMR (600 MHz, $\left.\mathrm{CDCl}_{3}\right):( \pm)-\mathbf{1 1}$

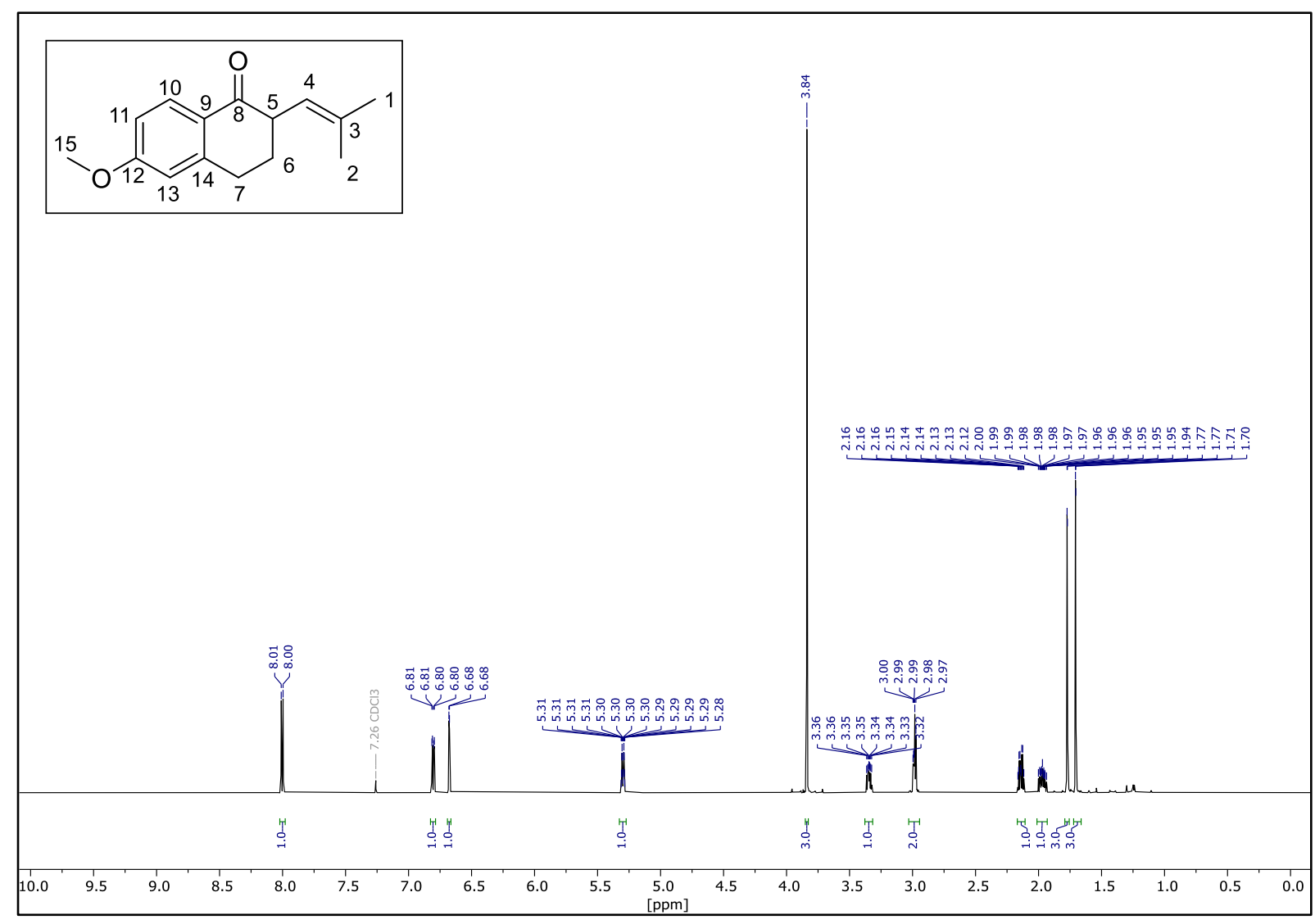

${ }^{13} \mathrm{C}$ NMR (151 MHz, $\left.\mathrm{CDCl}_{3}\right):( \pm)-11$
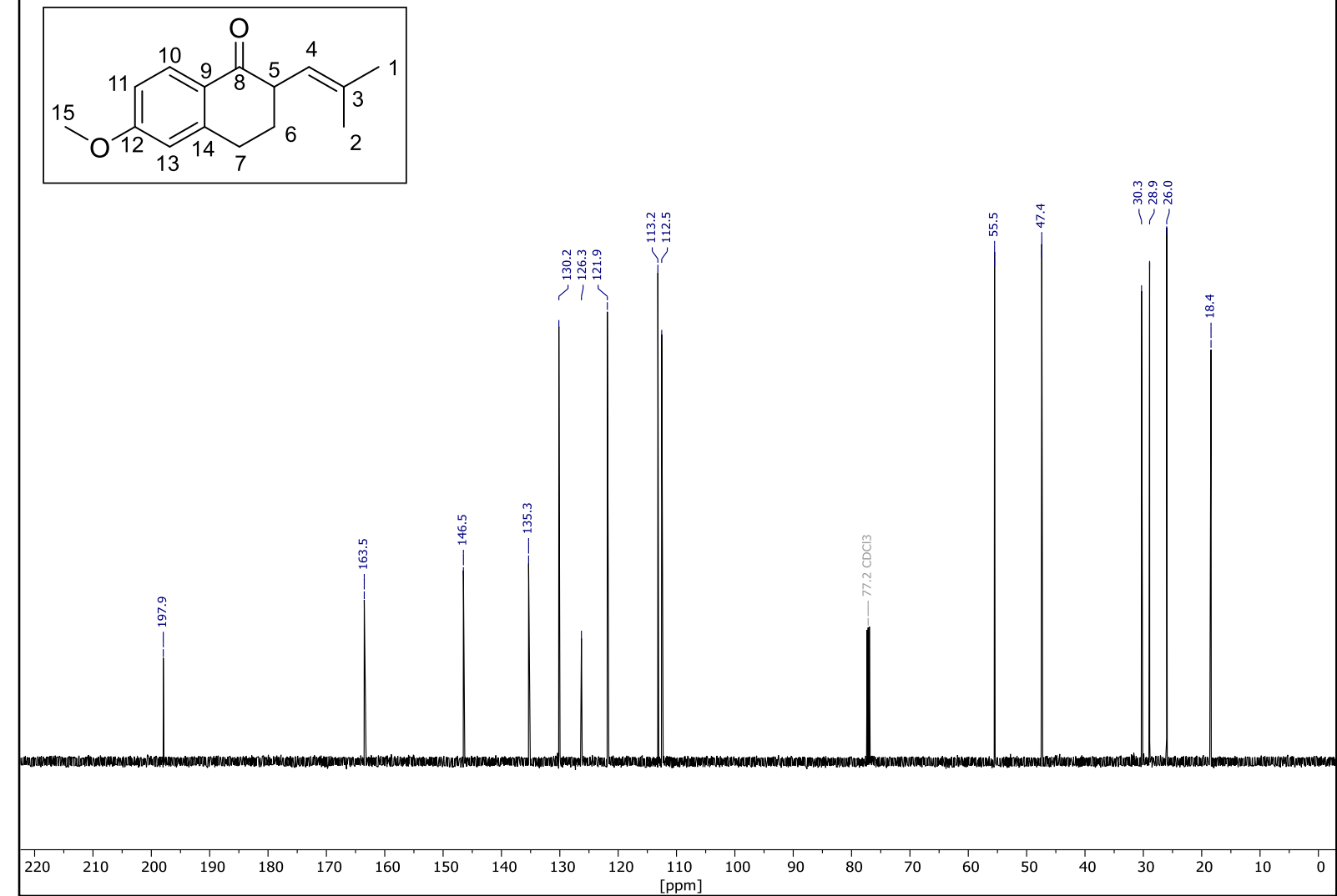
${ }^{1} \mathrm{H}$ NMR (400 MHz, $\left.\mathrm{CDCl}_{3}\right):( \pm)-12$

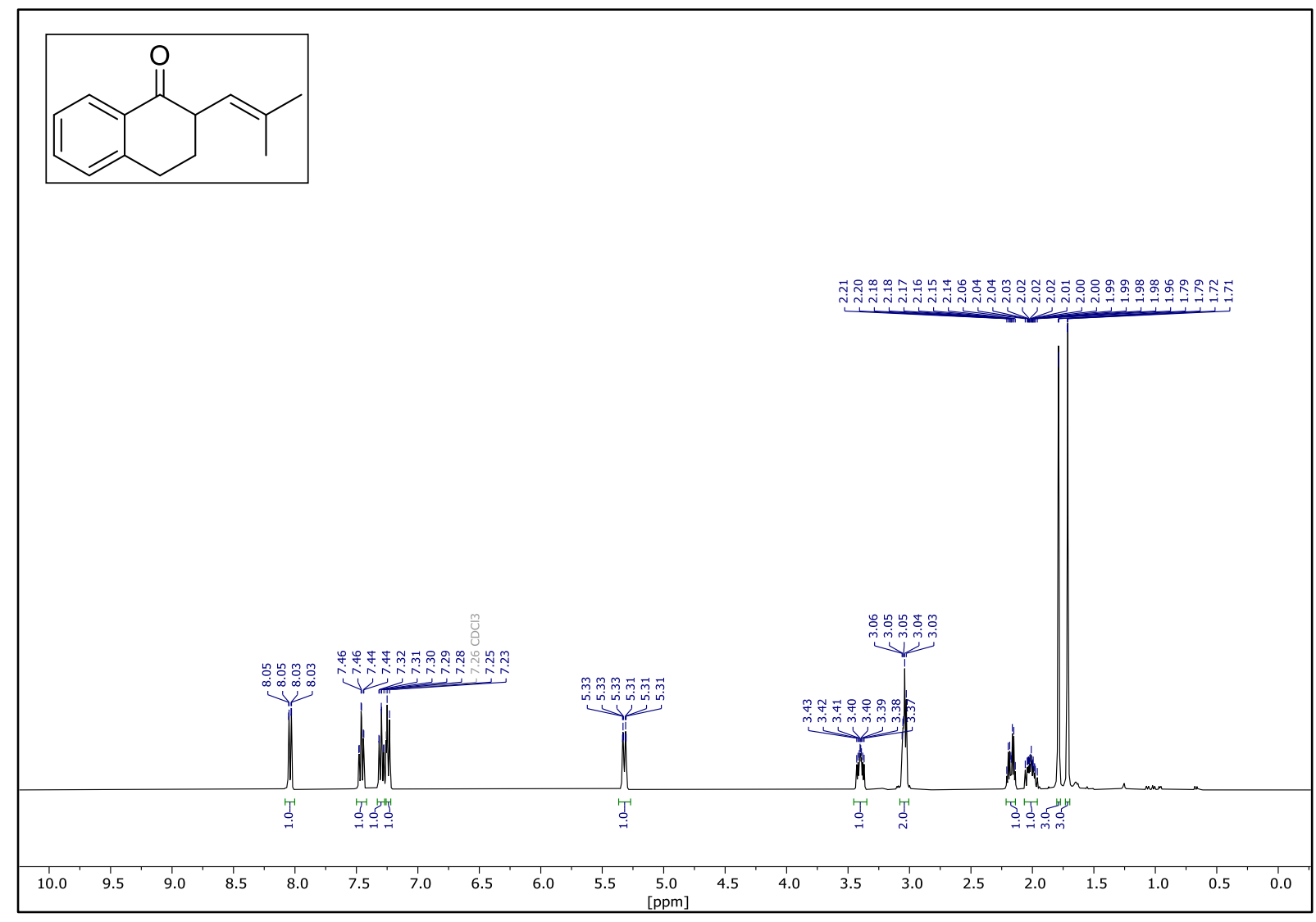

${ }^{1} \mathrm{H}$ NMR (500 MHz, $\left.\mathrm{CDCl}_{3}\right):( \pm)-\mathbf{1 3}$

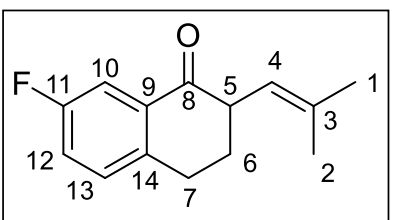

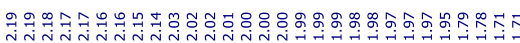

(1)

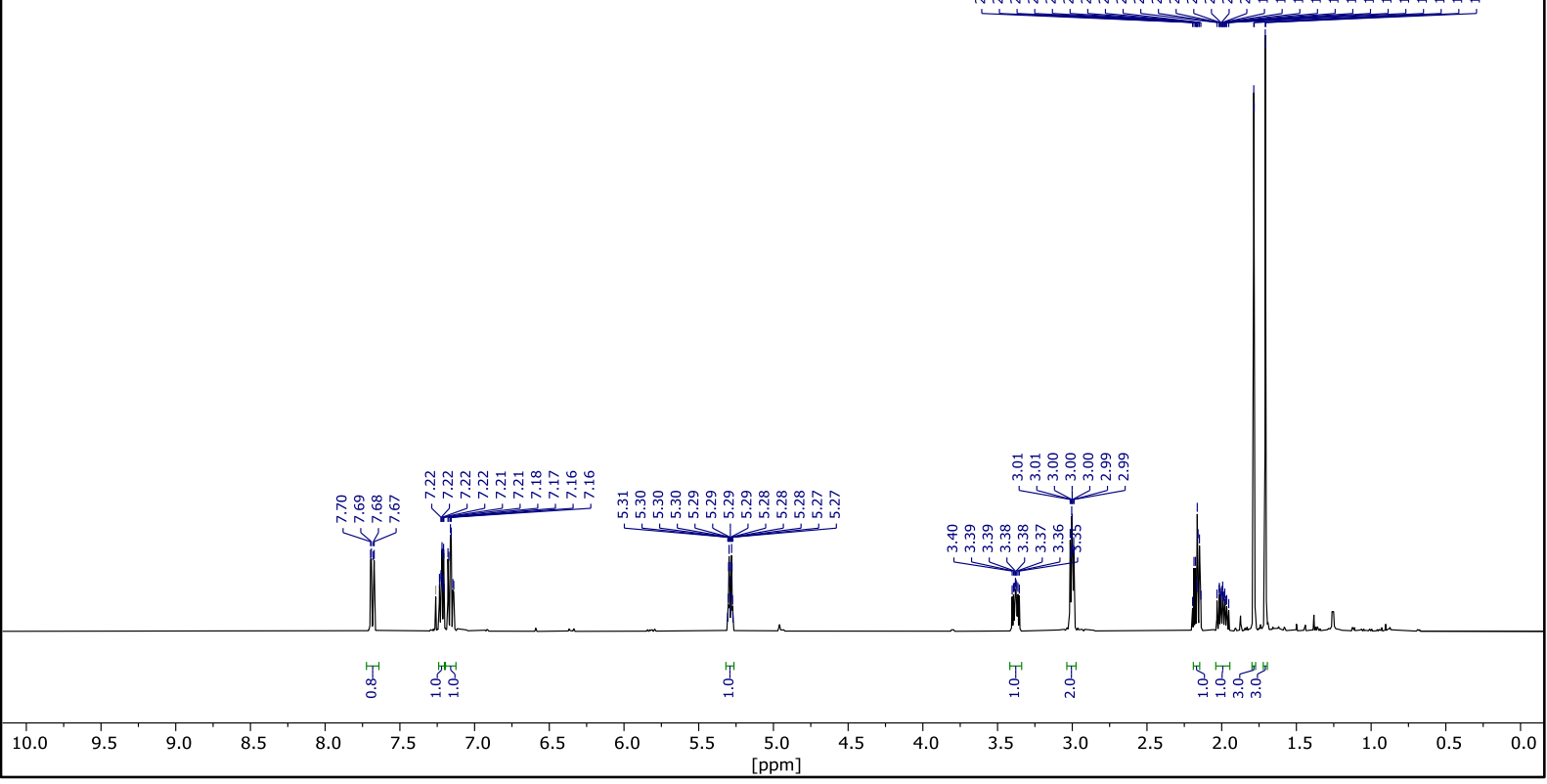


${ }^{13} \mathrm{C}$ NMR (126 MHz, $\left.\mathrm{CDCl}_{3}\right):( \pm)-13$

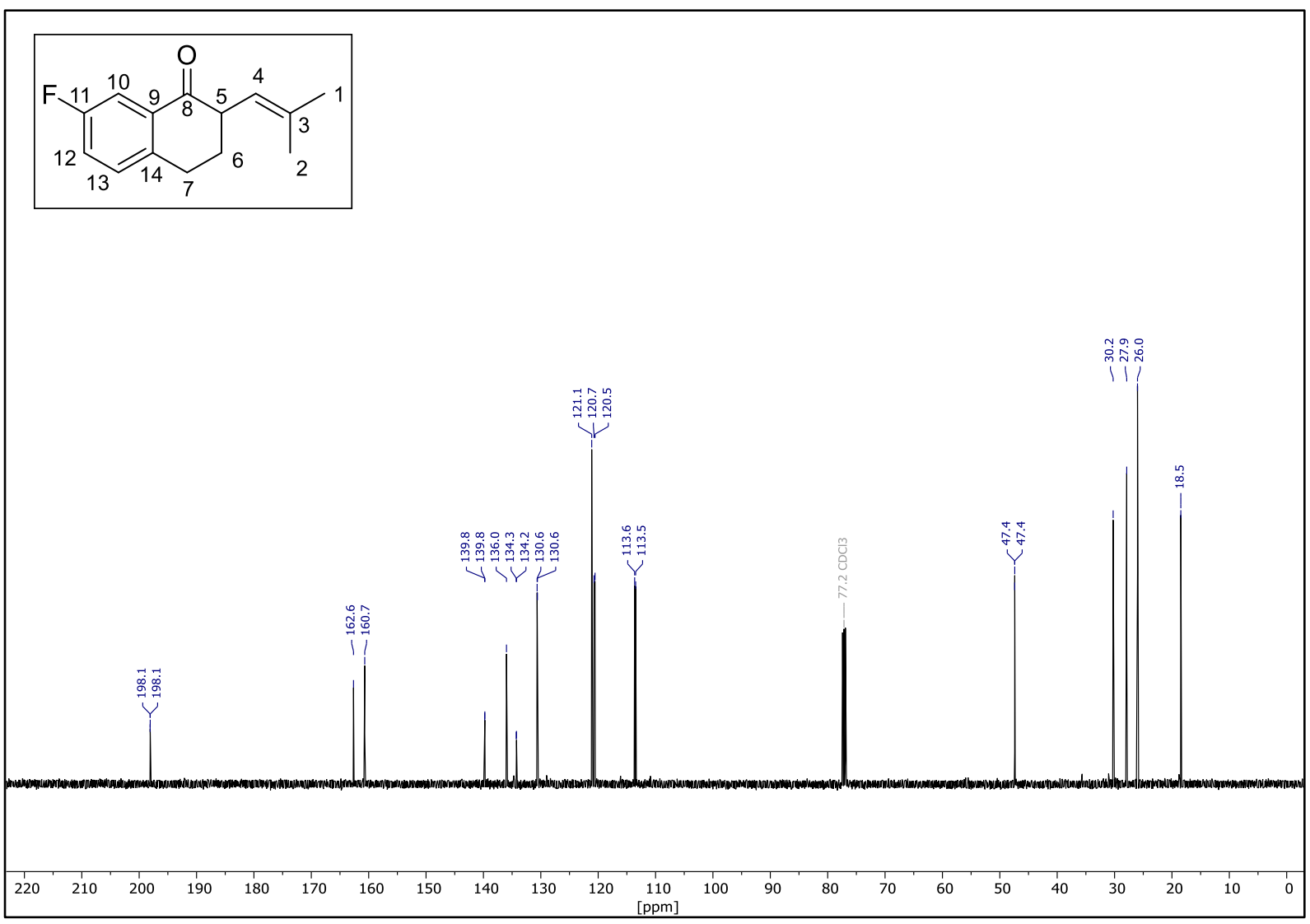

${ }^{19} \mathrm{~F}$ NMR $\left(470 \mathrm{MHz}, \mathrm{CDCl}_{3}\right):( \pm)-13$

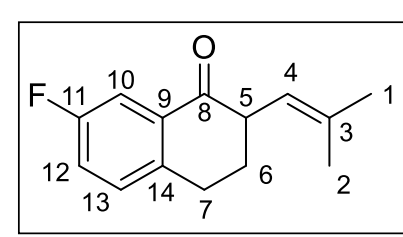

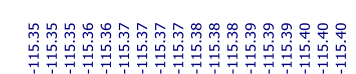

$\begin{array}{lllllllllllllllllllllllllllllllllllll}-92 & -94 & -96 & -98 & -100 & -102 & -104 & -106 & -108 & -110 & -112 & -114 & -116 & -118 & -120 & -122 & -124 & -126 & -128 & -130 & -132 & -134 & -136 & -138 & -140 & -142 & -144 & -146 & -148 & -150 & -15\end{array}$ 
${ }^{1} \mathrm{H}$ NMR (500 MHz, $\left.\mathrm{CDCl}_{3}\right):( \pm)-14$
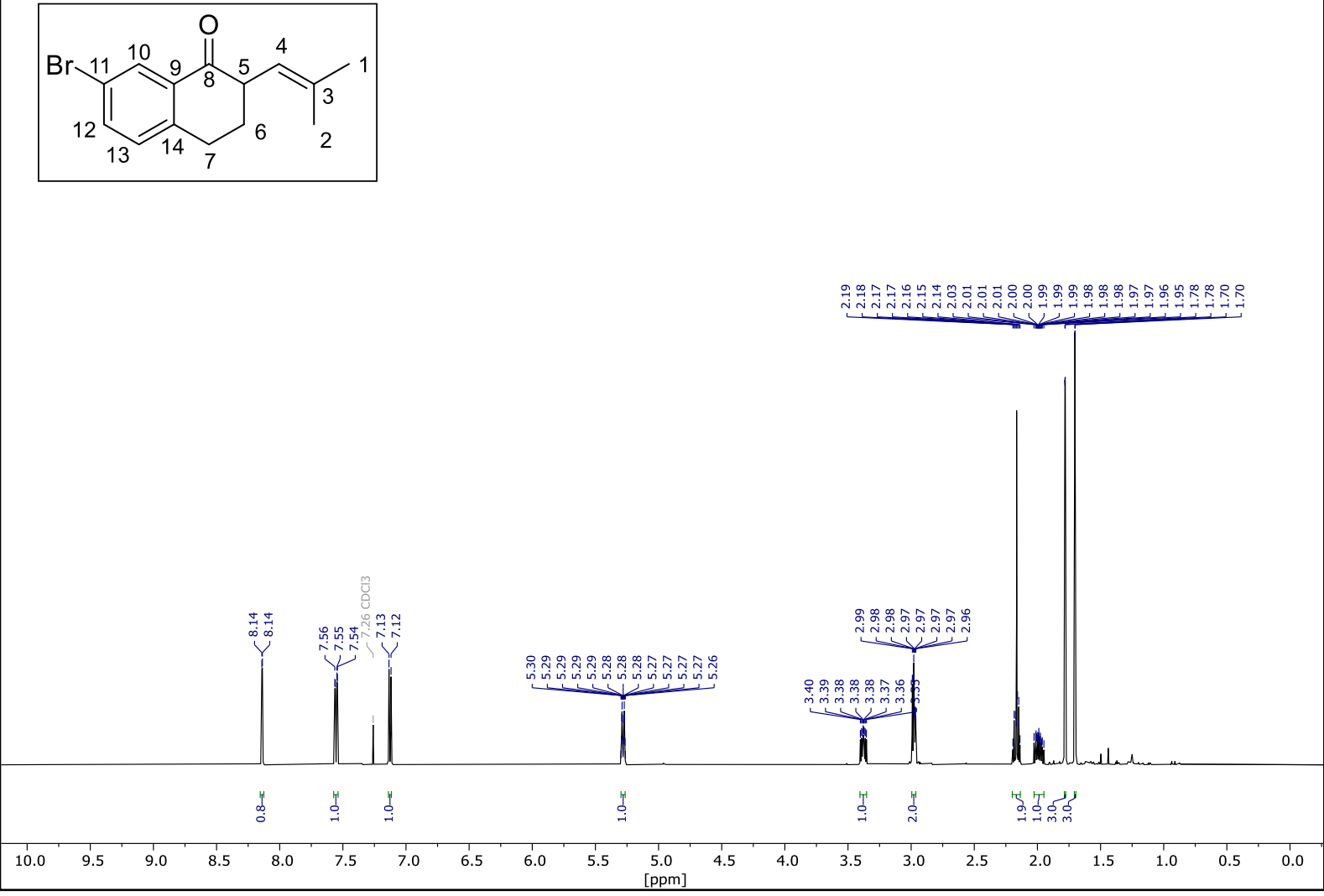

${ }^{13} \mathrm{C}$ NMR (126 MHz, $\left.\mathrm{CDCl}_{3}\right):( \pm)-14$
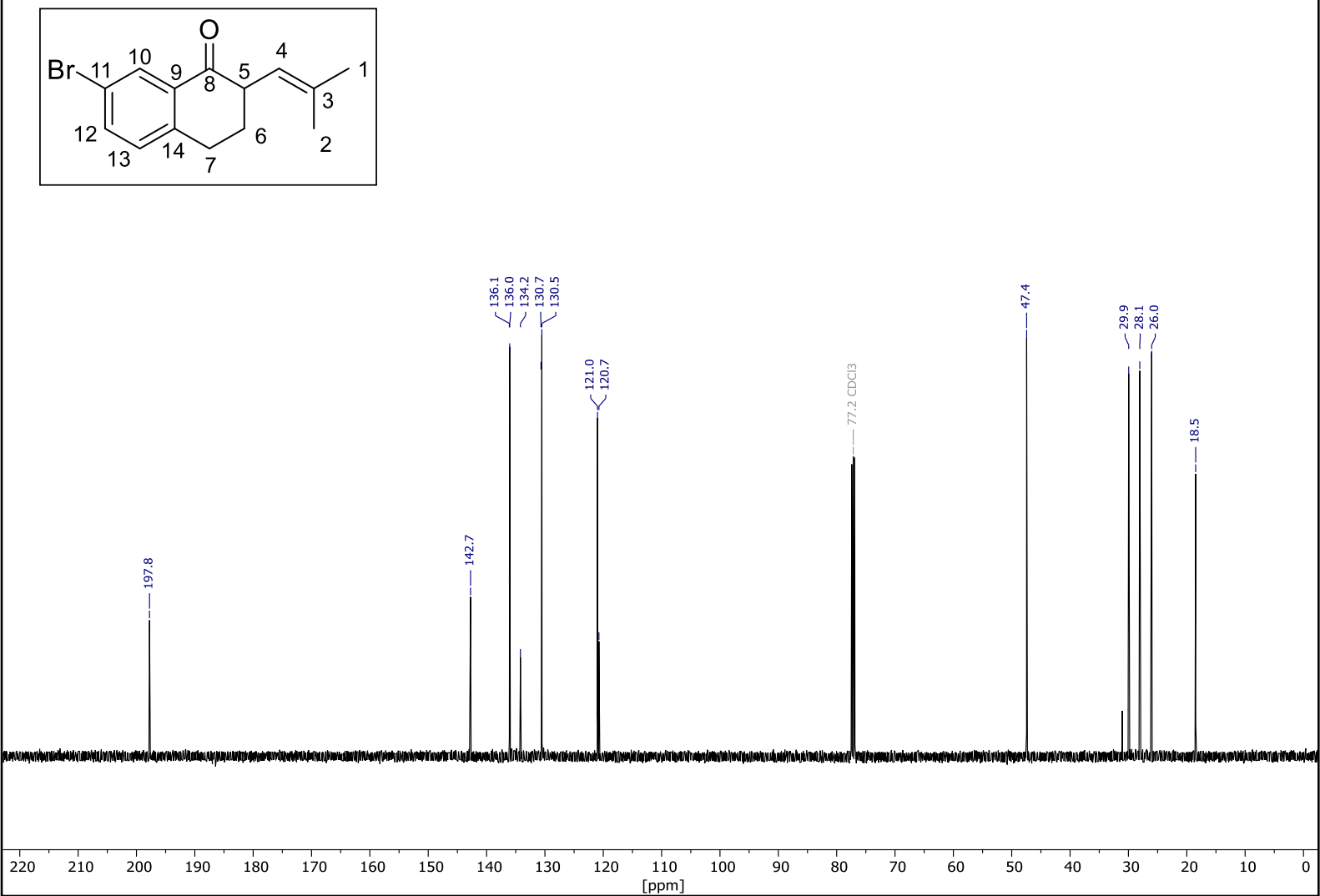
${ }^{1} \mathrm{H}$ NMR (600 MHz, $\left.\mathrm{CDCl}_{3}\right):( \pm)-15$

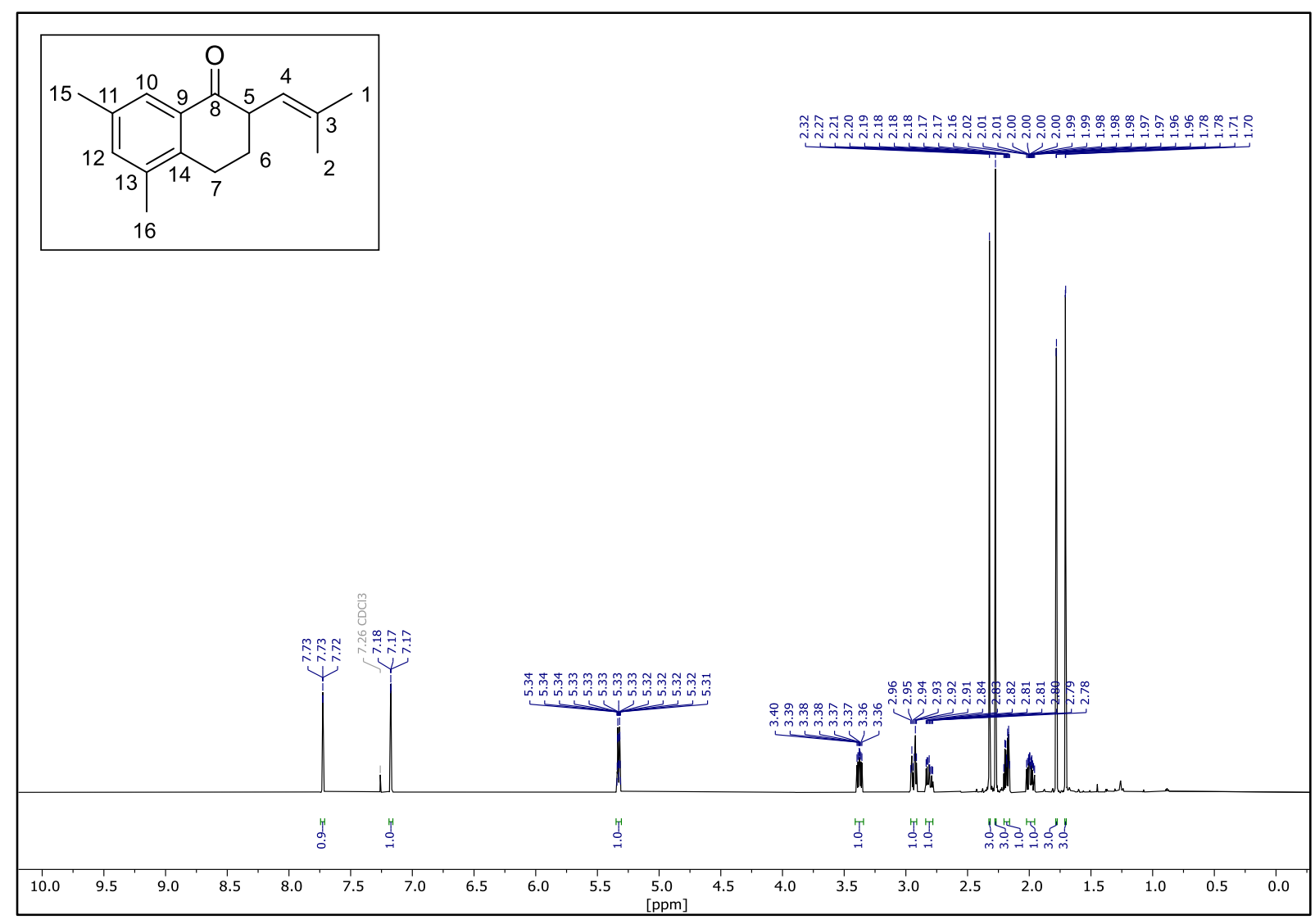

${ }^{13} \mathrm{C}$ NMR (151 MHz, $\left.\mathrm{CDCl}_{3}\right):( \pm)-\mathbf{1 5}$

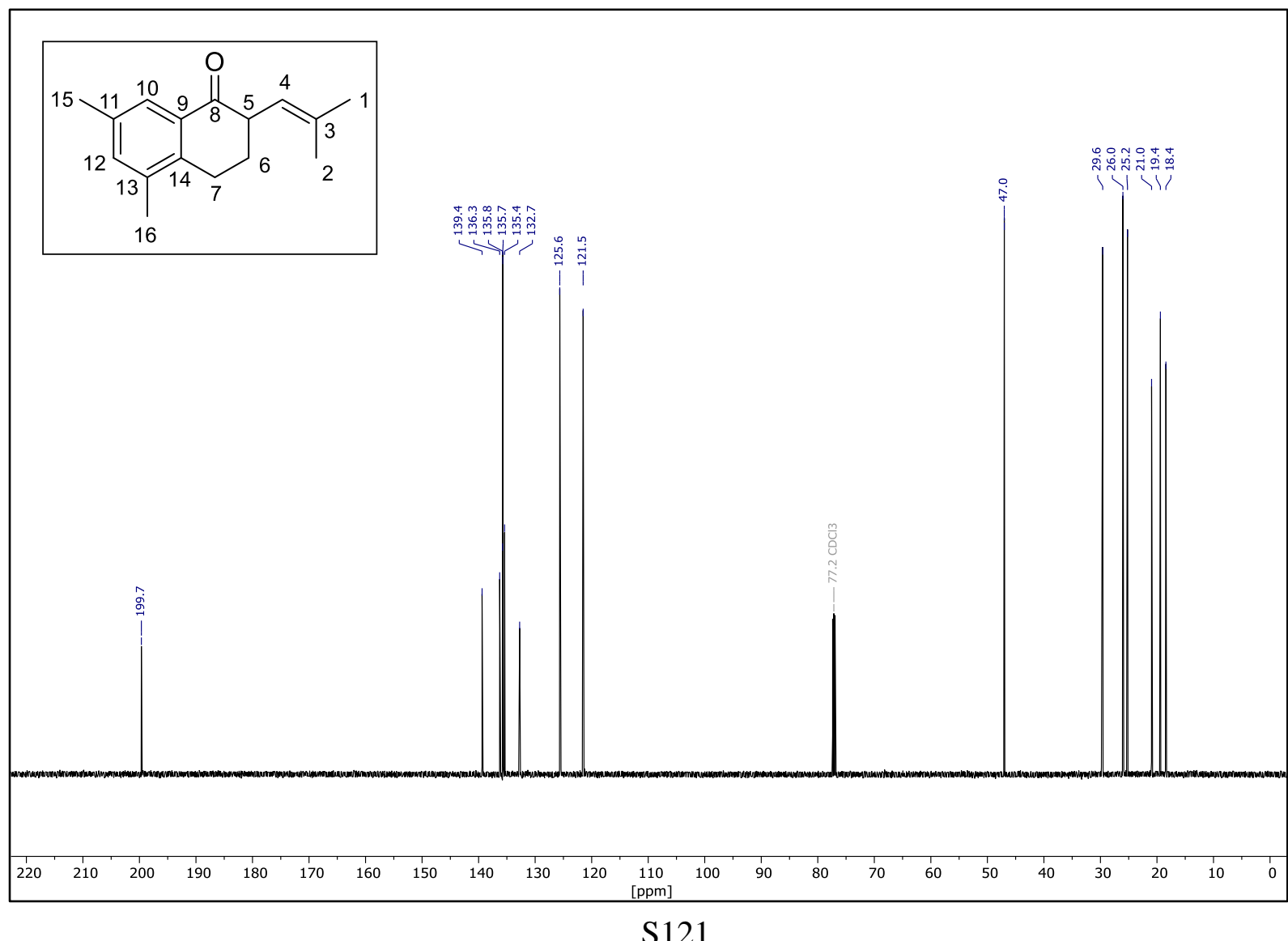


${ }^{1} \mathrm{H}$ NMR (600 MHz, $\left.\mathrm{CDCl}_{3}\right)$ : ( \pm )-16

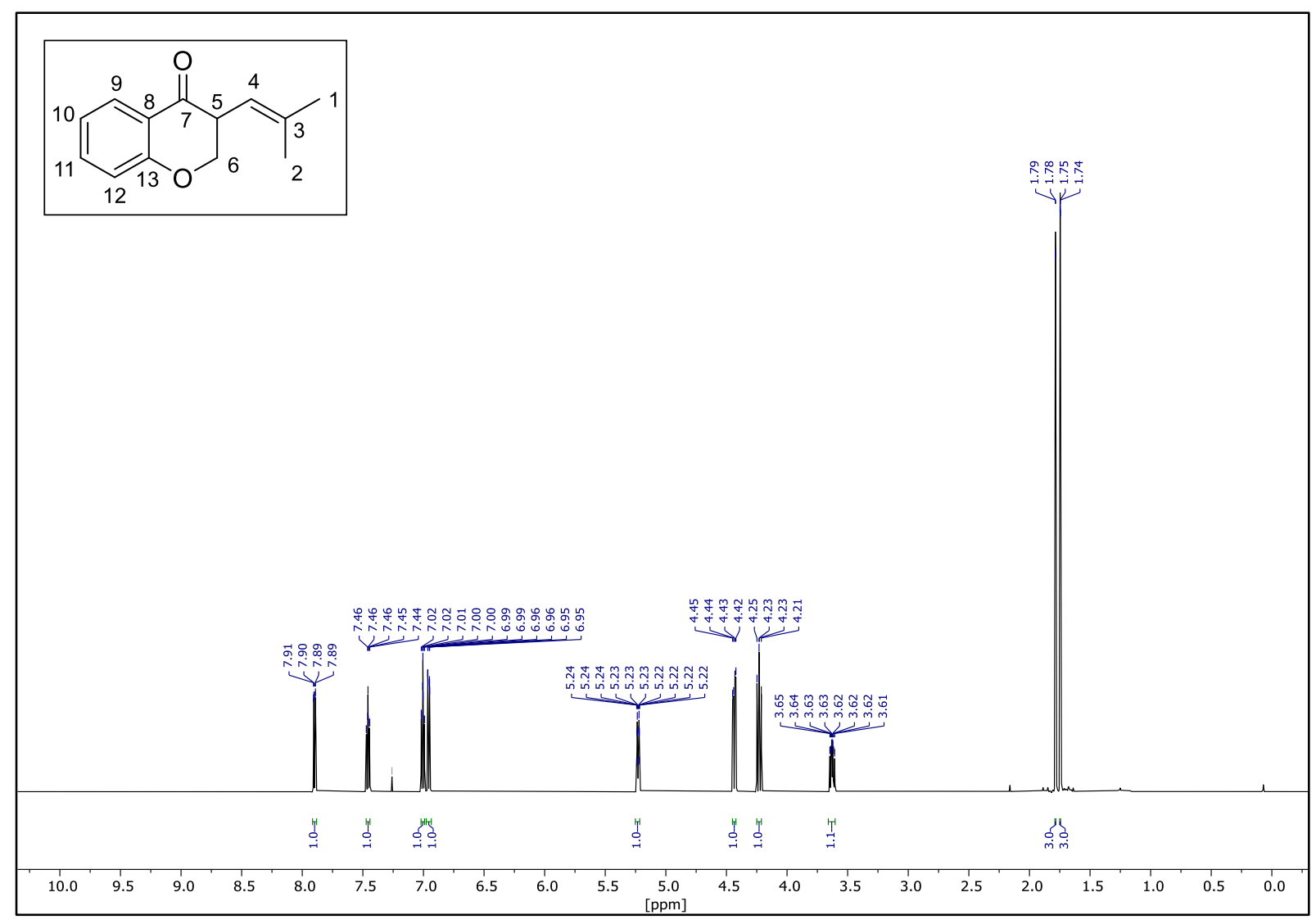

${ }^{13} \mathrm{C}$ NMR $\left(151 \mathrm{MHz}, \mathrm{CDCl}_{3}\right):( \pm)-16$
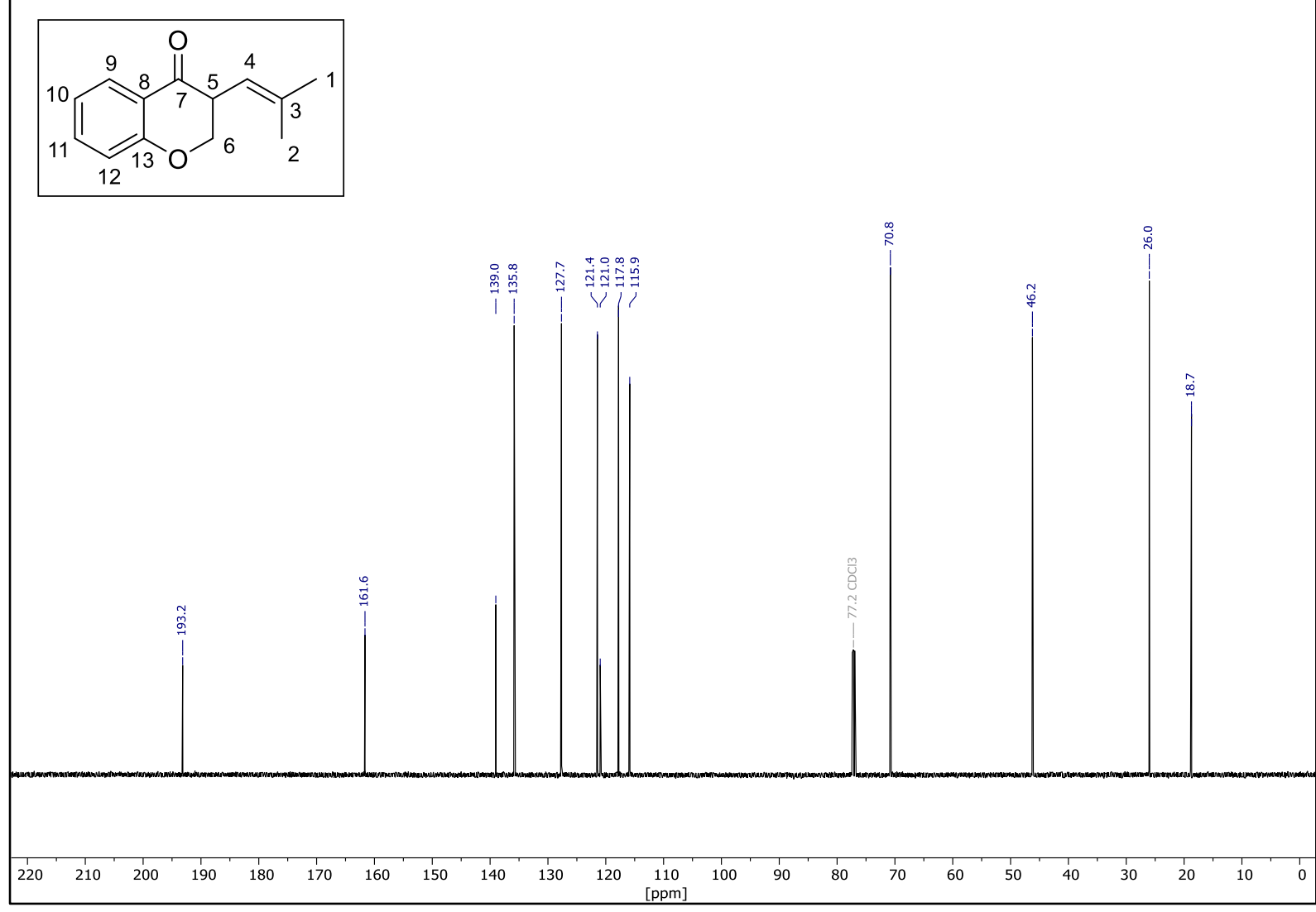
${ }^{1} \mathrm{H}$ NMR (500 MHz, $\left.\mathrm{CDCl}_{3}\right)$ : ( \pm )-17

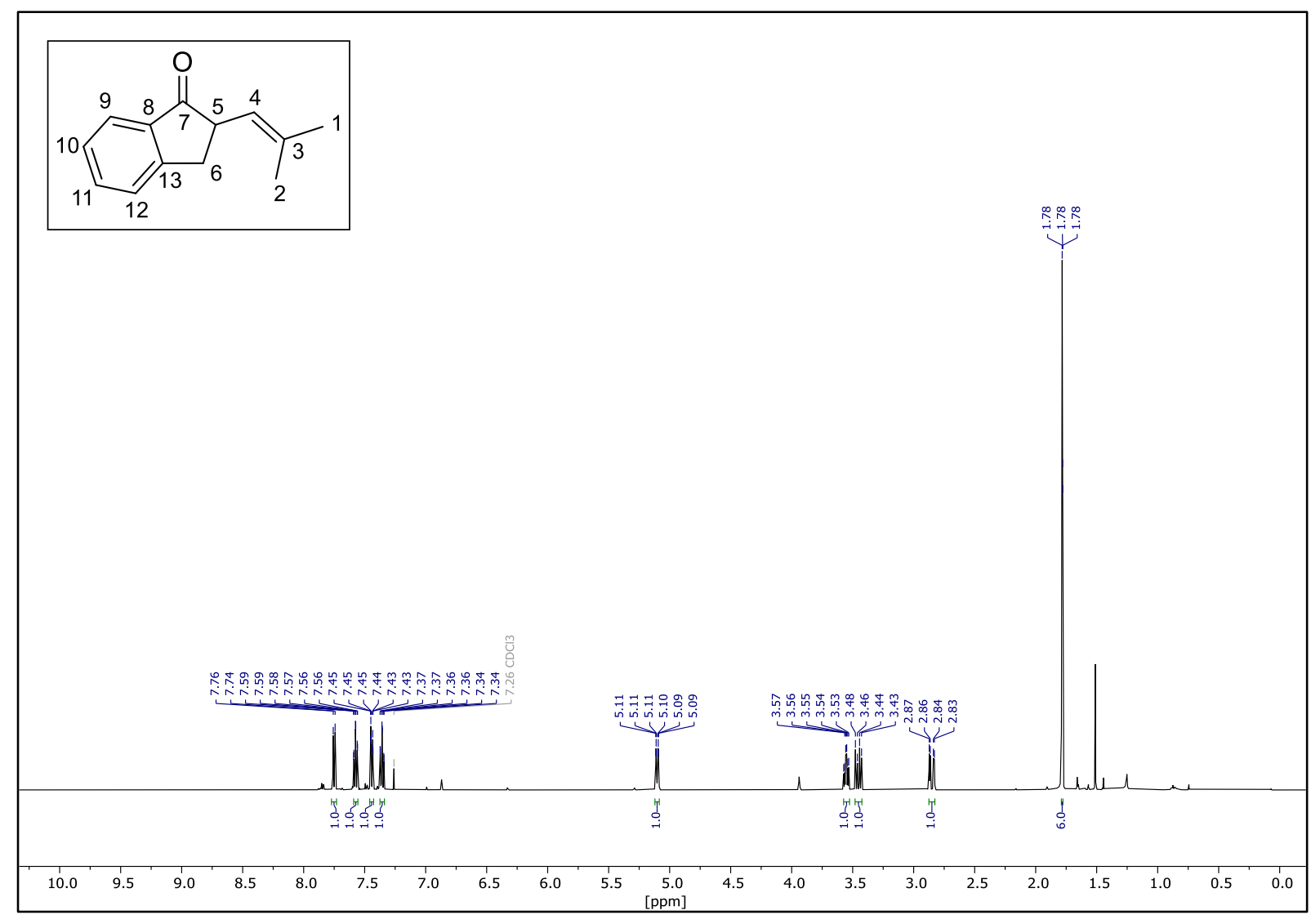

${ }^{13} \mathrm{C}$ NMR $\left(126 \mathrm{MHz}, \mathrm{CDCl}_{3}\right):( \pm)-17$

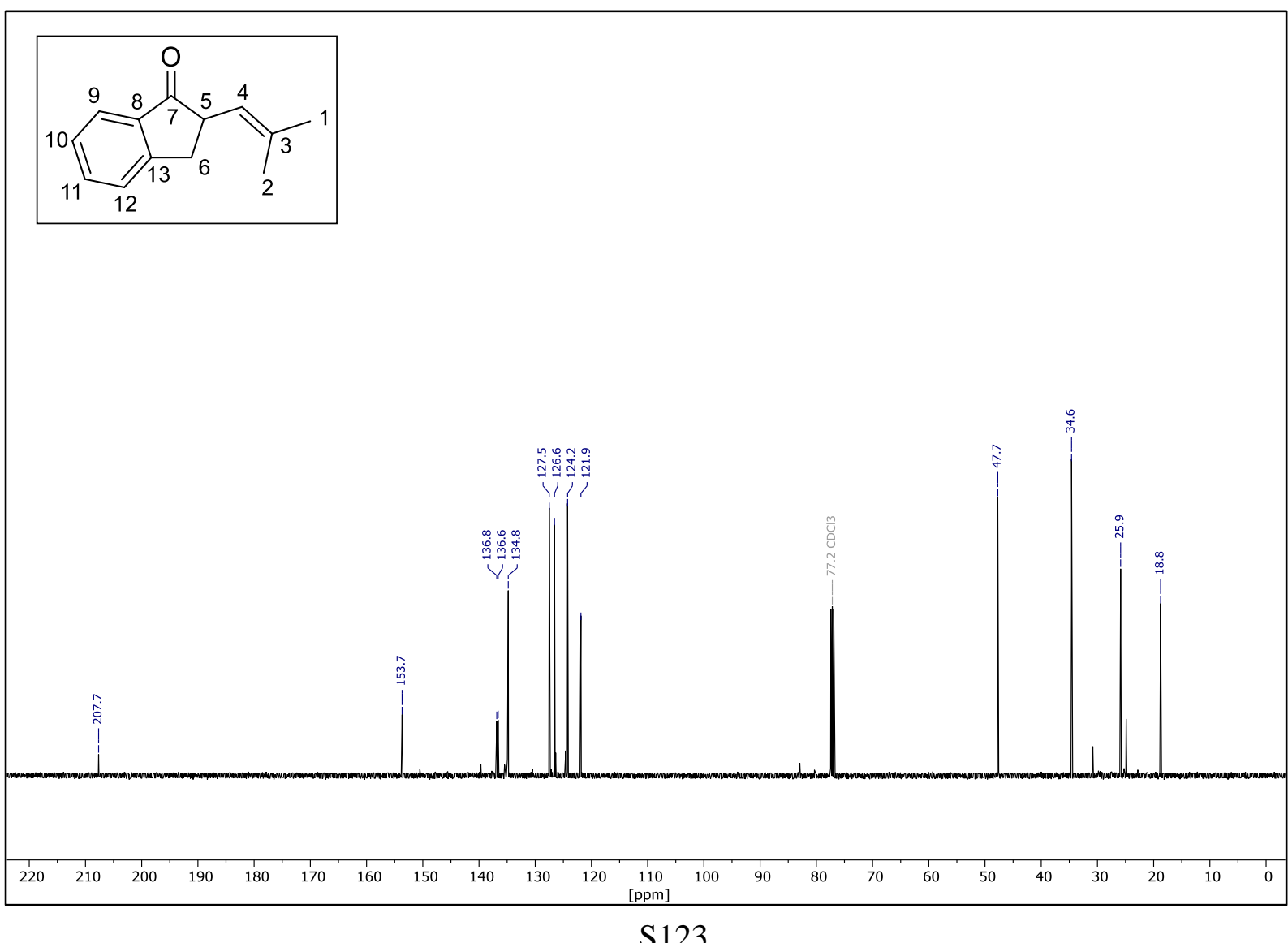


${ }^{1} \mathrm{H}$ NMR (600 MHz, $\left.\mathrm{CDCl}_{3}\right)$ : ( \pm )-18

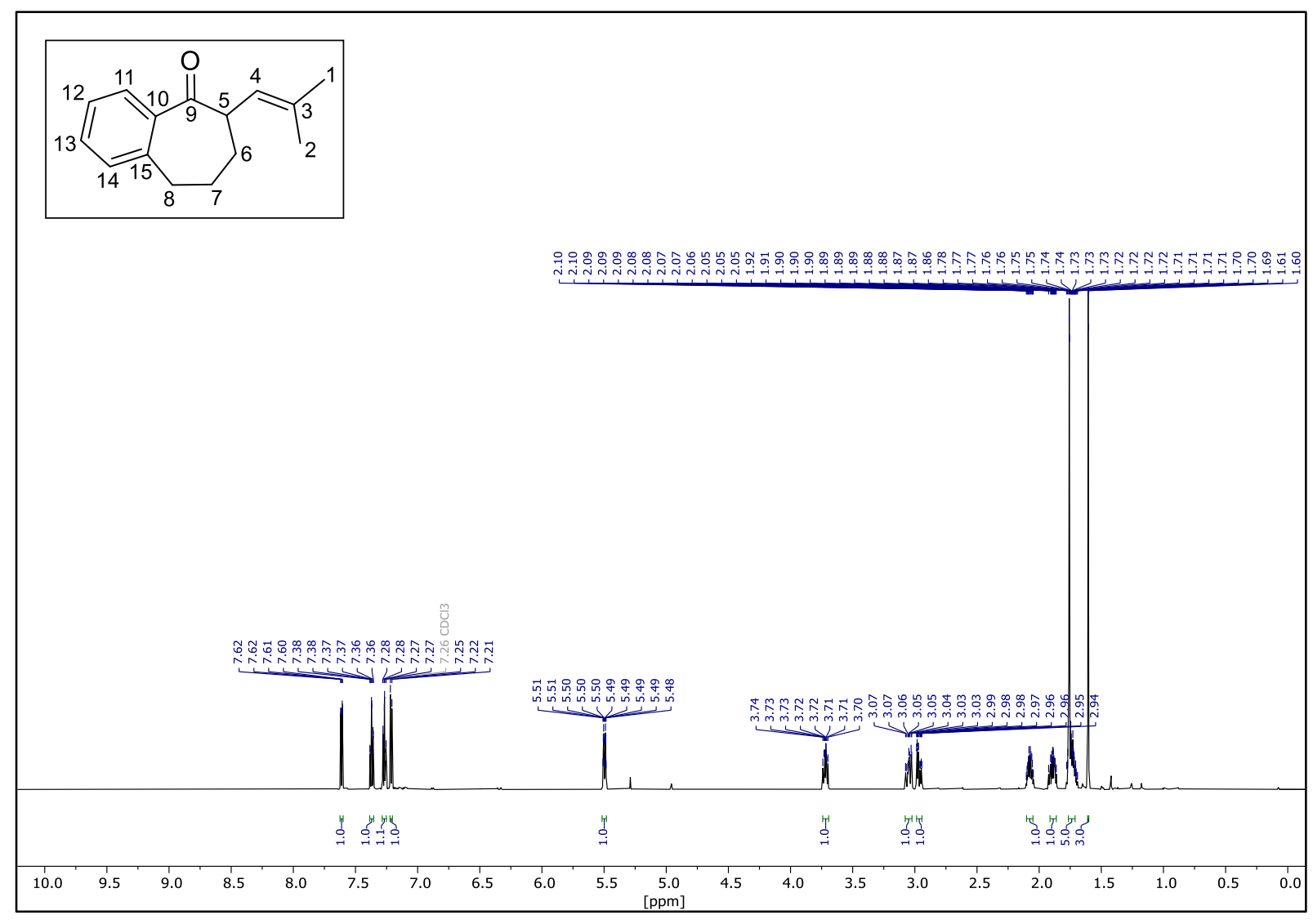

${ }^{13} \mathrm{C}$ NMR $\left(151 \mathrm{MHz}, \mathrm{CDCl}_{3}\right):( \pm)-18$
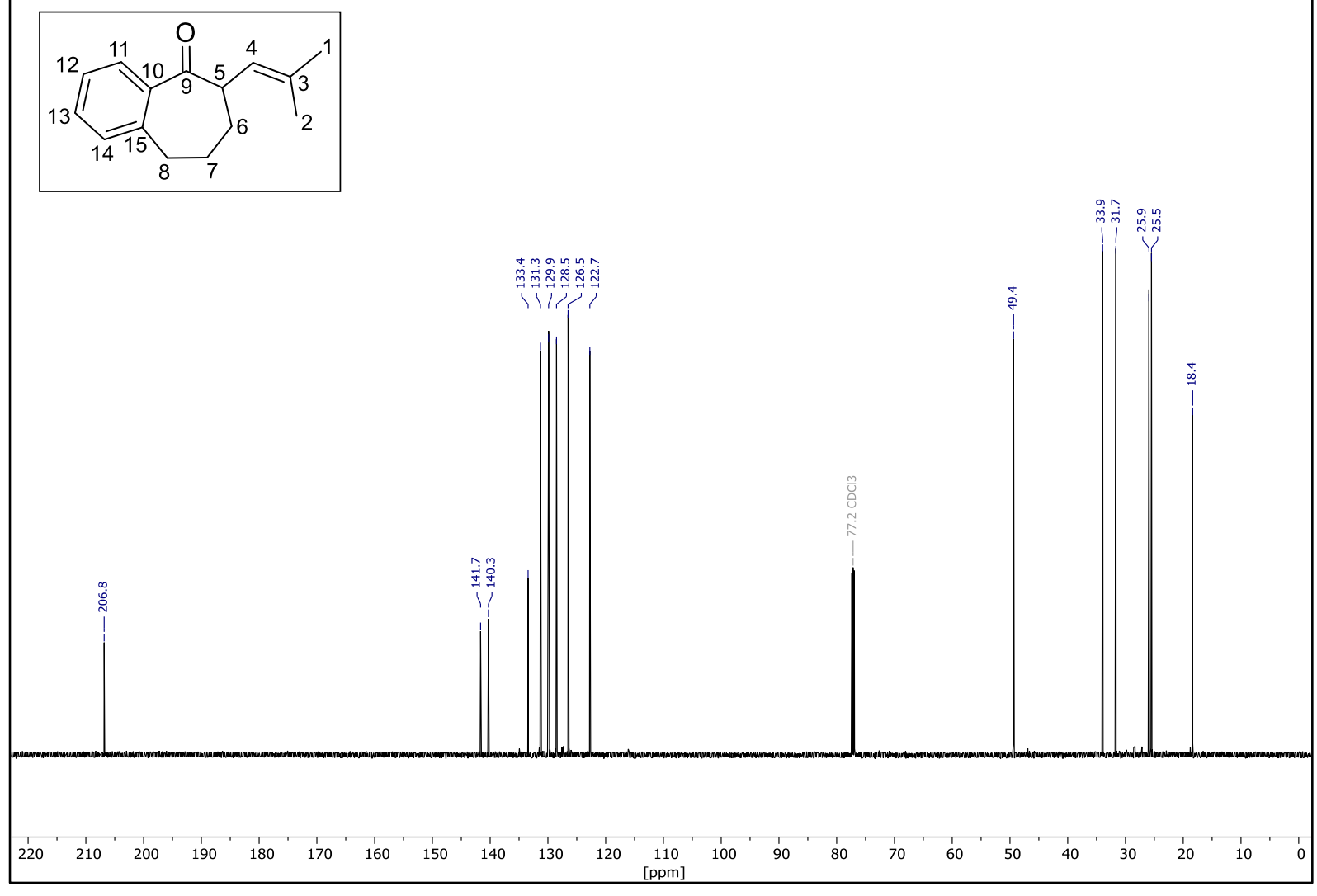
${ }^{1} \mathrm{H}$ NMR (400 MHz, $\left.\mathrm{CDCl}_{3}\right)$ : ( \pm )-19

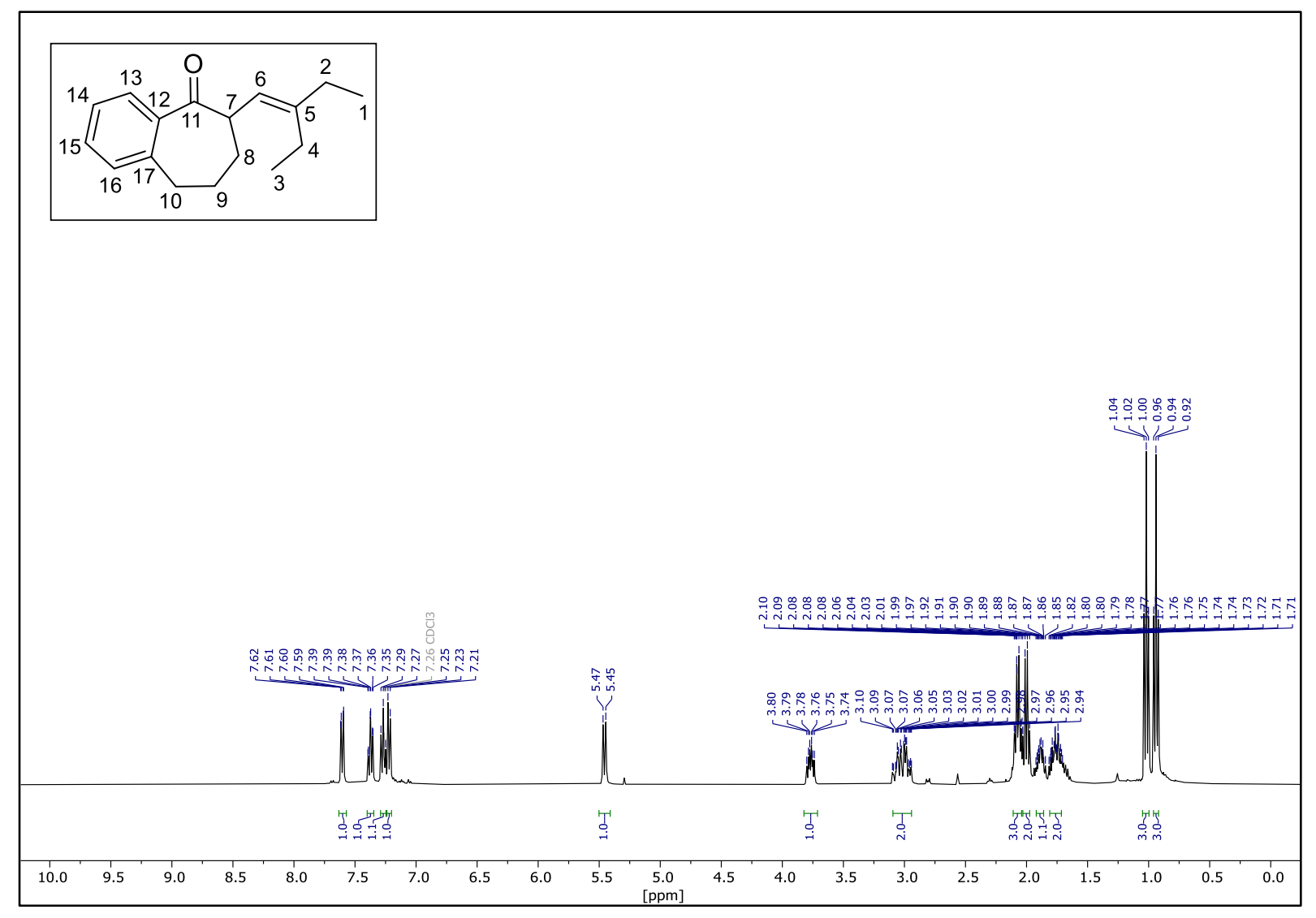

${ }^{13} \mathrm{C}$ NMR $\left(151 \mathrm{MHz}, \mathrm{CDCl}_{3}\right):( \pm)-19$

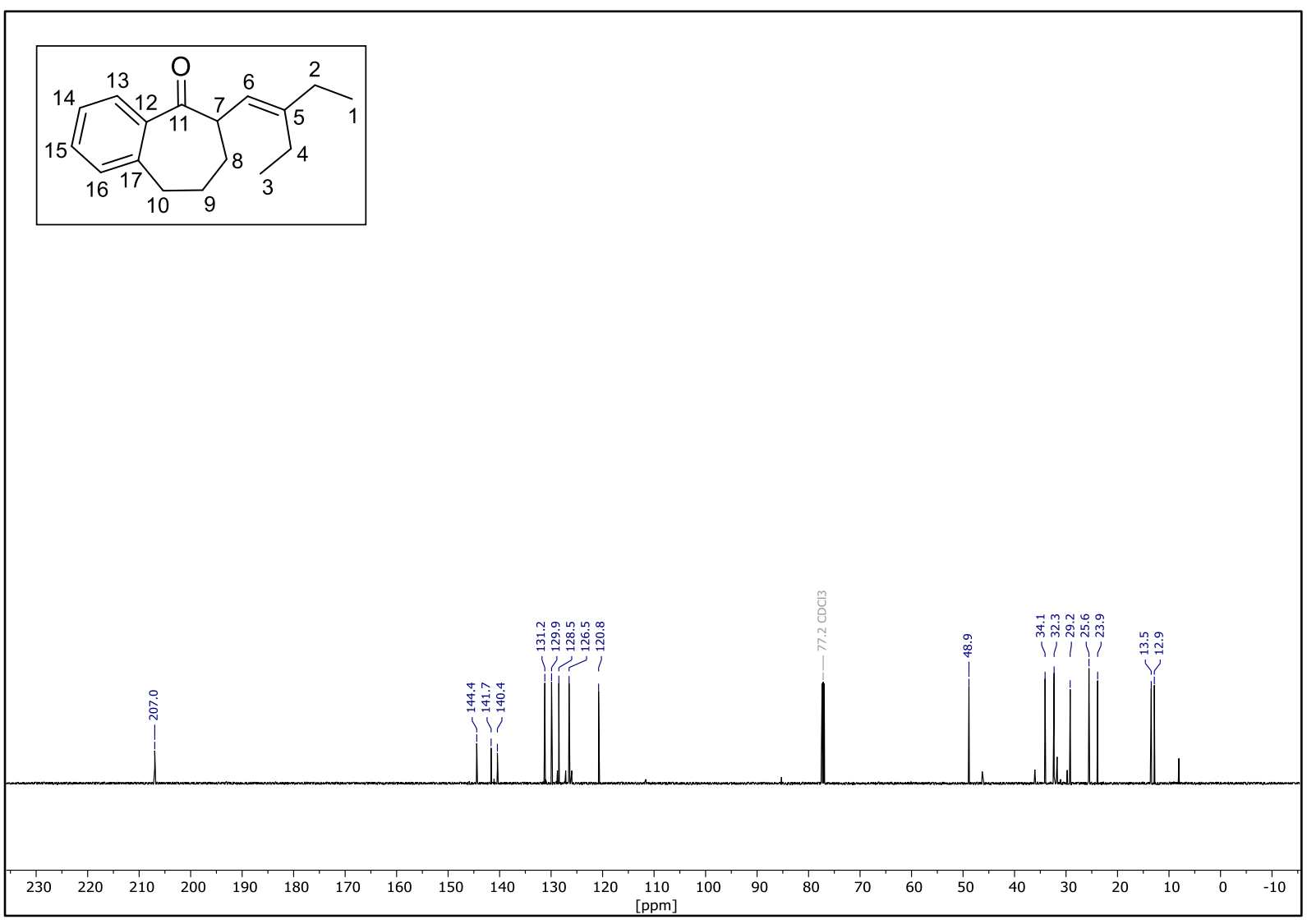


${ }^{1} \mathrm{H}$ NMR (500 MHz, $\left.\mathrm{CDCl}_{3}\right)$ : ( \pm )-20

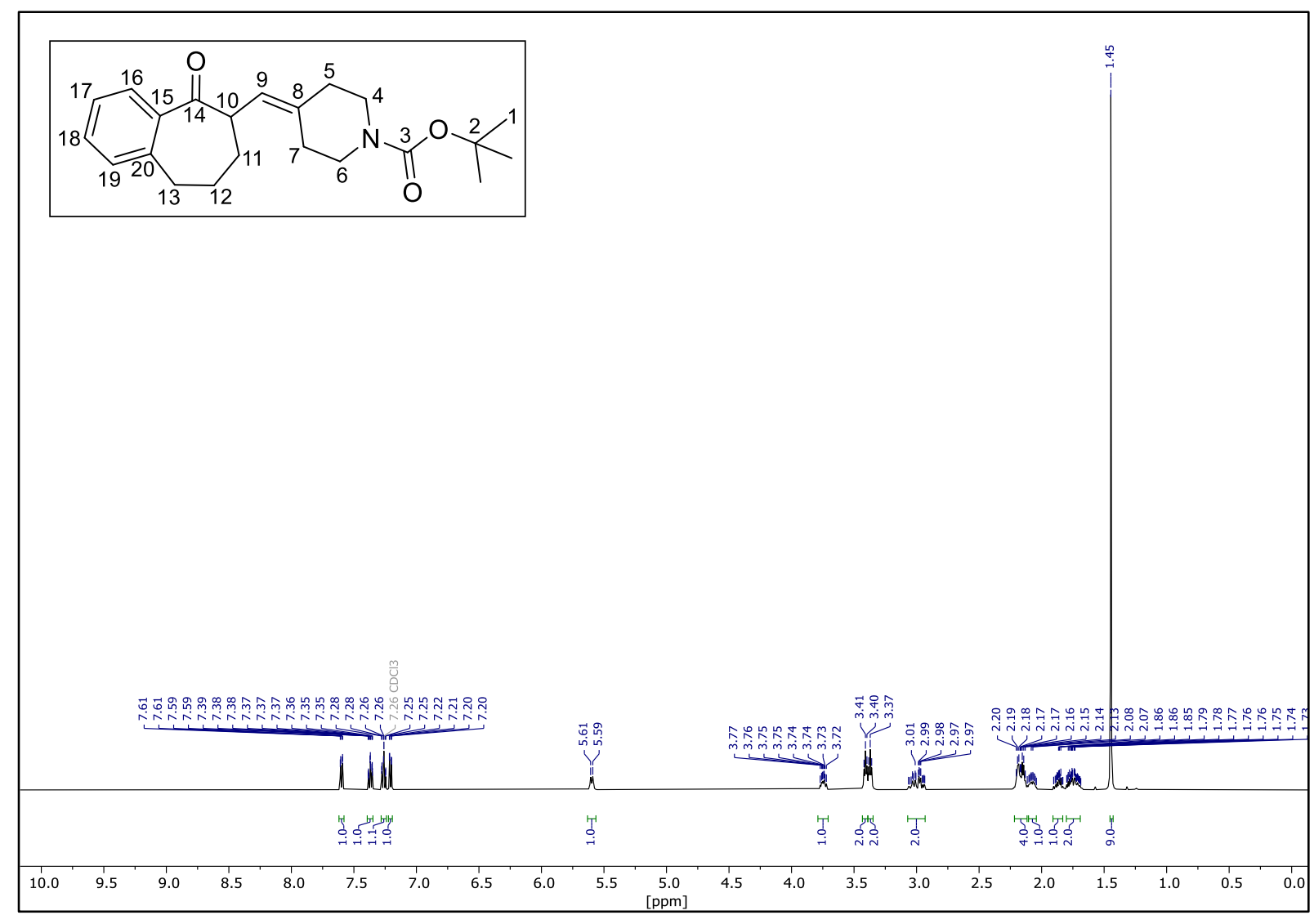

${ }^{13} \mathrm{C}$ NMR (126 MHz, $\left.\mathrm{CDCl}_{3}\right):( \pm)-20$

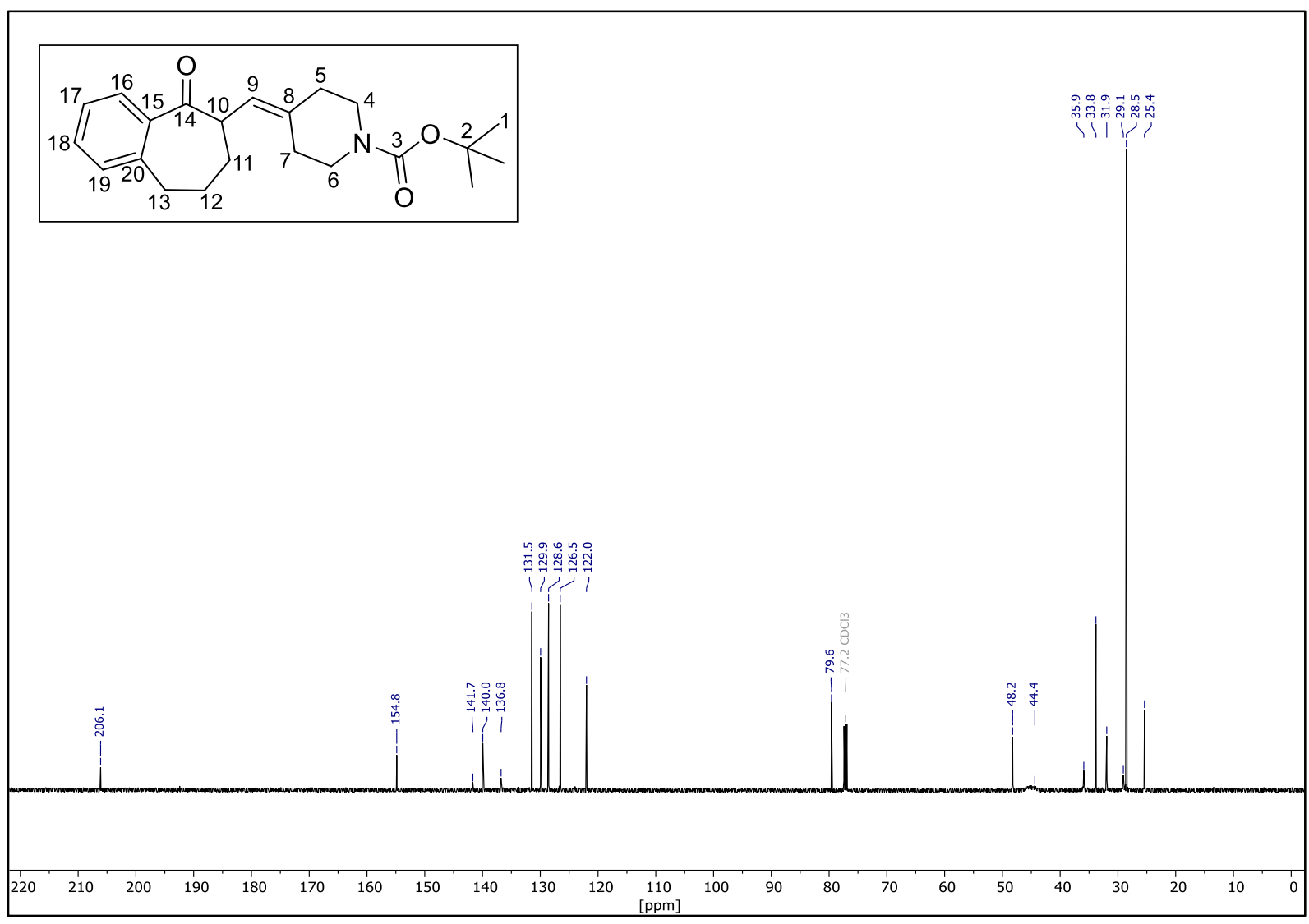


${ }^{1} \mathrm{H}$ NMR (500 MHz, $\left.\mathrm{CDCl}_{3}\right):( \pm)-21$
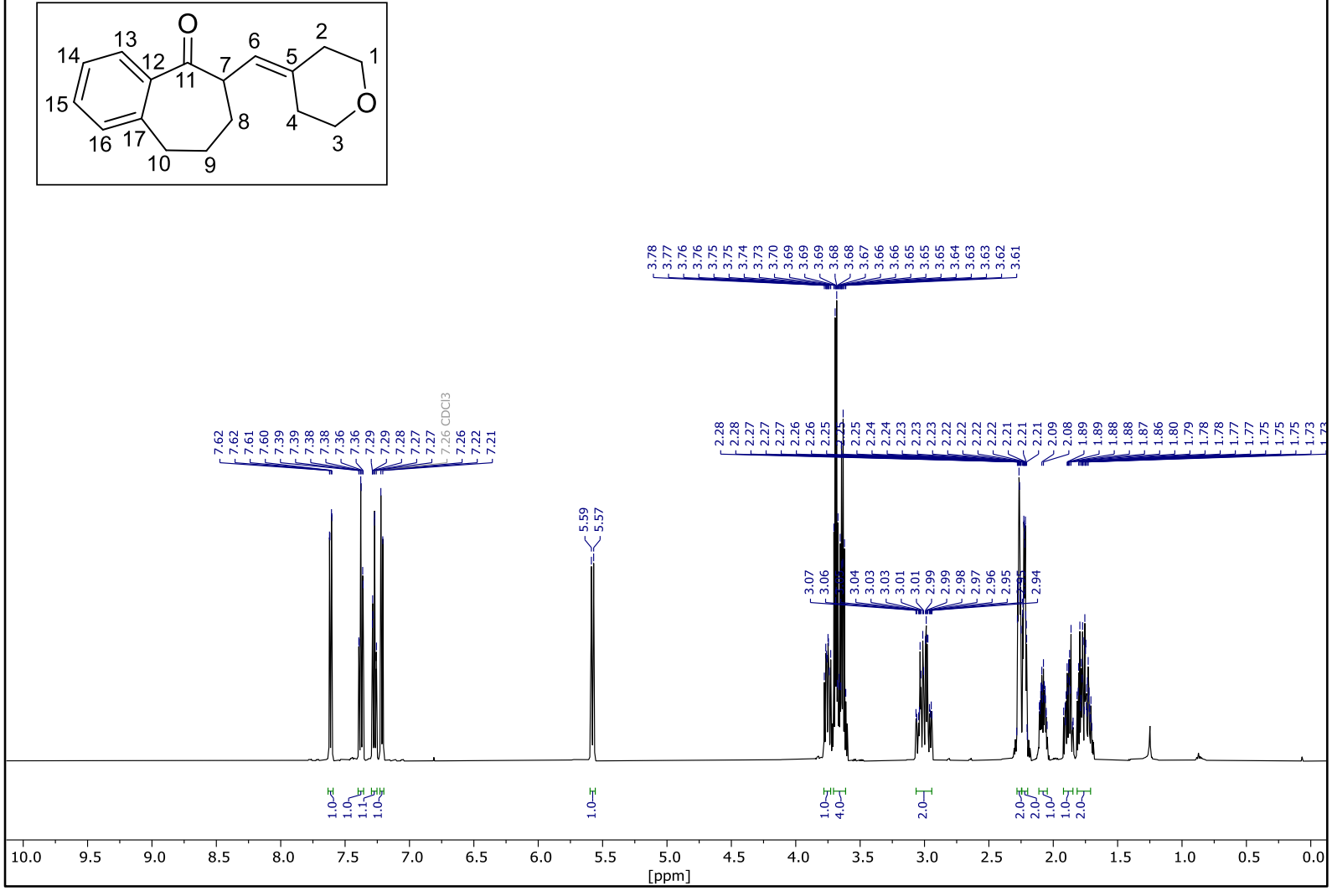

$\left.{ }^{13} \mathrm{C} \mathrm{NMR} \mathrm{(126} \mathrm{MHz,} \mathrm{CDCl}_{3}\right):( \pm)-21$
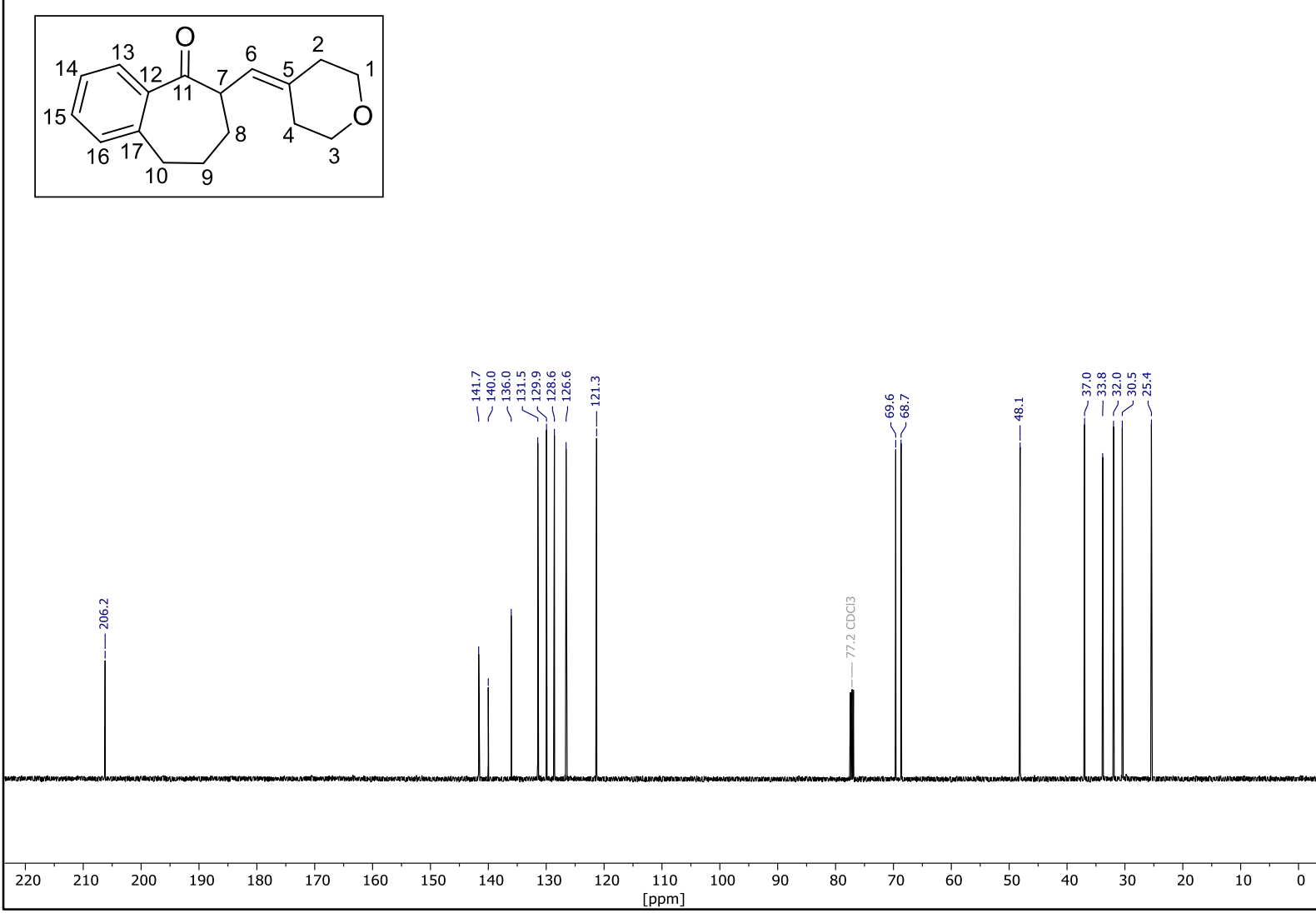


\section{References}

[1] Fulmer, G. R.; Miller, A. J. M.; Sherden, N. H.; Gottlieb, H. E.; Nudelman, A.; Stoltz, B. M.; Bercaw, J. E.; Goldberg; K. I. NMR Chemical Shifts of Trace Impurities: Common Laboratory Solvents, Organics, and Gases in Deuterated Solvents Relevant to the Organometallic Chemist. Organometallics 2010, 29, 2176-2179.

[2] Rhee, J. U.; Krische, M. J. Alkynes as Synthetic Equivalents to Stabilized Wittig Reagents: Intra- and Intermolecular Carbonyl Olefinations Catalyzed by $\mathrm{Ag}(\mathrm{I}), \mathrm{BF}_{3}$, and $\mathrm{HBF}_{4}$. Org. Lett., 2005, 7, 2493-2495.

[3] Singhal, N.; Koner, A. L.; Mal, P.; Venugopalan, P.; Nau, W. M.; Moorthy, J. N. Diastereomer-Differentiating Photochemistry of $\beta$-Arylbutyrophenones: Yang Cyclization versus Type II Elimination. J. Am. Chem. Soc., 2005, $127,14375-14382$.

[4] Miranda, P. O.; Diaz, D. D.; Padron, J. I.; Ramirez, M. A.; Martin, V. S. Fe(III) Halides as Effective Catalysts in Carbon-Carbon Bond Formation: Synthesis of 1,5-Dihalo-1,4-dienes, $\alpha, \beta$-Unsaturated Ketones, and Cyclic Ethers. J. Org. Chem., 2005, 70, 57-62.

[5] Lu, S.; Bolm, C. Highly Enantioselective Synthesis of Optically Active Ketones by Iridium-Catalyzed Asymmetric Hydrogenation. Angew. Chem. Int. Ed., 2008, 47, 8920-8923.

[6] Tian, F.; Yao, D.; Liu, Y.; Xie, F.; Zhang, W. Iridium-Catalyzed Highly Enantioselective Hydrogenation of Exocyclic $\alpha, \beta$-Unsaturated Carbonyl Compounds. Adv. Synth. Cat., 2010, 352, $1841-1845$.

[7] Barman, P.; Cantú Reinhard, F. G.; Bagha, U. K.; Kumar, D.; Sastri, C. V.; de Visser, S. P. Hydrogen by Deuterium Substitution in an Aldehyde Tunes the Regioselectivity by a Nonheme Manganese(III)-Peroxo Complex. Angew. Chem. Int. Ed., 2019, 58, 10639-10643.

[8] Rodriguez-Escrich, S.; Sola, L.; Jimeno, C.; Rodriguez-Escrich, C.; Pericas, M. A. Exploring Structural Diversity in Ligand Design: The Aminoindanol Case. Adv. Synth. Cat. 2008, 350, 2250-2260.

[9] Grigalunas, M.; Ankner, T.; Norrby, P.; Wiest, O.; Helquist, P. Palladium-Catalyzed Alkenylation of Ketone Enolates under Mild Conditions. Org. Lett. 2014, 16, 3970-3973.

[10] Zaid, Y.; Mboyi, C. D.; Drapeau, M. P.; Radal, L.; Chahdi, Fouad O.; Rodi, Y. K.; Ollevier, T.; Taillefer, M. Transition-Metal-Free $\alpha$-Vinylation of Enolizable Ketones with $\beta$-Bromostyrenes. Org. Lett. 2019, 21, 1564-1568.

[11] Tsuda, T.; Kiyoi, T.; Saegusa, T. Nickel(0)-catalyzed hydroacylation of alkynes with aldehydes to $\alpha, \beta$-enones J. Org. Chem. 1990, 55, 2554-2558.

[12] Hansch, C.; Leo, A.; R. Taft, R. W. A survey of Hammett substituent constants and resonance and field parameters. Chem. Rev. 1991, 91, 165-195.

[13] TURBOMOLE V7.4 2019, a development of University of Karlsruhe and Forschungszentrum Karlsruhe GmbH, 1989-2007, TURBOMOLE GmbH, since 2007; available from http://www.turbomole.com

[14] Tao, J.; Perdew, J. P.; Staroverov, V. N.; Scuseria, G. E. Climbing the Density Functional Ladder: Nonempirical Meta-Generalized Gradient Approximation Designed for Molecules and Solids. Phys. Rev. Lett., 2003, 91, 146401.

[15] a) Grimme, S.; Antony, J.; Ehrlich, S.; Krieg, H. A consistent and accurate ab initio parametrization of density functional dispersion correction (DFT-D) for the 94 elements H-Pu J. Chem. Phys. 2010, 132, 154104; b) Grimme, S.; Ehrlich, S.; Goerigk, L. Effect of the damping function in dispersion corrected density functional theory. $J$. Comput. Chem. 2011, 32, 1456-1465.

[16] Weigend, F.; Ahlrichs. R. Balanced basis sets of split valence, triple zeta valence and quadruple zeta valence quality for $\mathrm{H}$ to Rn: Design and assessment of accuracy. Phys. Chem. Chem. Phys. 2005, 7, 3297-3305. 
[17] Grimme, S. Supramolecular Binding Thermodynamics by Dispersion-Corrected Density Functional Theory. Chem. Eur. J. 2012, 18, 9955-9964.

[18] Zhao, Y.; Truhlar, D. G. Design of Density Functionals That Are Broadly Accurate for Thermochemistry, Thermochemical Kinetics, and Nonbonded Interactions. J. Phys. Chem. A 2005, 109, 5656-5667.

[19] a) Klamt, A. Conductor-like Screening Model for Real Solvents: A New Approach to the Quantitative Calculation of Solvation Phenomena. J. Phys. Chem. 1995, 99, 2224-2235. b) Eckert, F.; Klamt, A. COSMOtherm, Version C3.0; COSMOlogic GmbH \& Co. KG, Leverkusen, Germany, 2013.

[20] Bannwarth, C.; Ehlert, S.; Grimme, S. GFN2-xTB-An Accurate and Broadly Parametrized Self-Consistent Tight-Binding Quantum Chemical Method with Multipole Electrostatics and Density-Dependent Dispersion Contributions. J. Chem. Theory. Comput. 2019, 15, 1652-1671.

[21] Grimme, S. Exploration of Chemical Compound, Conformer, and Reaction Space with Meta-Dynamics Simulations Based on Tight-Binding Quantum Chemical Calculations. J. Chem. Theory Comput. 2019, 15, 28472862.

[22] Chai, J.-D.; Head-Gordon, M. Systematic optimization of long-range corrected hybrid density functionals. $J$. Chem. Phys. 2008, 128, 084106.

[23] Neese, F. The ORCA program system, Wiley Interdisciplinary Reviews: Computational Molecular Science, 2012, 2, 73-78. 